Portland State University

PDXScholar

$1-1-2011$

\title{
Differences in Sexual Dimorphism and Influences of Sexual Dichromatism on Crypsis Among Populations of the Jumping Spider Habronattus oregonensis
}

Jason Bazzano

Portland State University

Follow this and additional works at: https://pdxscholar.library.pdx.edu/open_access_etds Let us know how access to this document benefits you.

\section{Recommended Citation}

Bazzano, Jason, "Differences in Sexual Dimorphism and Influences of Sexual Dichromatism on Crypsis Among Populations of the Jumping Spider Habronattus oregonensis" (2011). Dissertations and Theses. Paper 272.

https://doi.org/10.15760/etd.272

This Thesis is brought to you for free and open access. It has been accepted for inclusion in Dissertations and Theses by an authorized administrator of PDXScholar. Please contact us if we can make this document more accessible: pdxscholar@pdx.edu. 
Differences in Sexual Dimorphism and Influences of Sexual

Dichromatism on Crypsis Among Populations of

the Jumping Spider Habronattus oregonensis

by

Jason Bazzano

A thesis submitted in partial fulfillment of the requirements for the degree of

\author{
Master of Science \\ in \\ Biology \\ Thesis Committee: \\ Susan Masta, Chair \\ Suzanne Estes \\ Michael Murphy \\ Portland State University \\ 2011
}




\begin{abstract}
Crypsis can be an important mechanism of predator avoidance for organisms. However, many species exhibit sexual dichromatism, in which the males possess a suite of colorations in order to attract female attention. The resulting differences in crypsis between the males and females can provide insight into the relative strengths of the sexually and naturally selective forces shaping the coloration of the organism, as well as clues regarding potential sensory biases of the selecting sex. In this study, I examine variation in the coloration of four Pacific Northwest populations of the sexually dimorphic and dichromatic polygynous species of jumping spider Habronattus oregonensis and compare the coloration of different body regions of the spiders to their habitats. I also investigate differences in relative size of a male sexual ornament, the enlarged first leg tibia. Field work for this study was conducted in June and July of 2009 . The three main foci of this study are 1) to compare the degree of color matching of females and their habitat to the degree of color matching of males and their habitat, evaluating whether sexual selection on males has reduced their degree of crypsis relative to that of females, 2 ) if there is indeed a difference in crypsis between the sexes, to gauge whether there are similar divergences from crypsis among the populations - both in the quantitative amount of divergences as well as the colorimetric direction of such divergences, and 3) whether there is any variation in sexual ornament size among populations. Male first leg tibia size is a sexual character that is presumably not influenced by habitat coloration; differences


in male tibia allometry among populations would provide supporting evidence for the hypothesis that sexual selection is indeed maintaining phenotypic differences among the populations, regardless of habitat location and color.

I found a high degree of conformity of hue and chroma between male and female spiders and their habitats, with three notable exceptions. The most extreme difference in coloration between spider and habitat was that of the Gorge and Siskiyou population male anteriors. The anteriors had proportionally less green and more ultraviolet reflectance than their habitat. Second, the Mt. Hood and Tillamook population male abdomens diverged from their habitat in a similar, although less pronounced manner to that of the Gorge and Siskiyou population male anteriors: they had proportionally less green and more UV reflectance. Third, female abdomens of all populations were highly variable in chroma, despite having hues that generally matched their habitat. Tibia area relative to body size of Gorge and Siskiyou population males was significantly smaller than that of Mt. Hood and Tillamook population males.

The lower level of background hue matching among males compared to females implies that sexual selection has directly conflicted with natural selection, resulting in impaired crypsis. While the reduced crypsis of the Gorge and Siskiyou population males is centered on their anterior (the primary body region presented to the females during courtship), the deviations from crypsis in the Mt. Hood and Tillamook population males are highest on their abdomen, although the degree of 
contrast is lower than that of the Gorge and Siskiyou population anteriors. These differences in coloration between the Mt. Hood and Tillamook population male abdomens and their habitats are in the same colorimetric direction as those of the Gorge and Siskiyou population anteriors and their habitat; this may indicate a sensory bias of the females, conserved in all four populations, selecting for male reflectance with a higher UV to green ratio. The fact that Mt. Hood and Tillamook population male abdomens have a more modest reduction in background matching compared to Gorge and Siskiyou population male anteriors may be due to the search methods of flying predators (e.g., spider wasps); the dorsum would presumably be more conspicuous to predators than the anterior, and would thus be subject to more intense selection for crypsis despite sexual selection to the contrary.

The variability of abdomen coloration of females of both morphs may indicate that selection for crypsis is less strong among females than among males. One possible reason for this would be if females spent less time in the exposed courtship habitat than males, a conclusion implied by a highly male-skewed sex ratio encountered during field collections.

Like the differences in coloration between different males of different populations, the significant differences in male tibia size also imply variability in the intensity of sexual selection. Relative importance of male coloration and tibia size may be weighted differently among populations, operating under similar constraints on reductions in survival accrued by developing these characters. 
The high degree of variation found among the populations implies that there is a degree of reproductive isolation among the chromatically and morphologically dissimilar populations. However, the similarity of the environments in which the populations existed, the close geographic proximity of some of the dissimilar populations, and the lack of any substantial geographic boundaries between the populations imply that this isolation is not maintained through extrinsic factors. Rather, it would seem that the interpopulational diversity is maintained by sexual selection. However, evidence from morphology and coloration suggest that the generation of this diversity is not evolving exclusively under sexual selection pressure, but rather is constrained to a degree by natural selection. 
Table of Contents

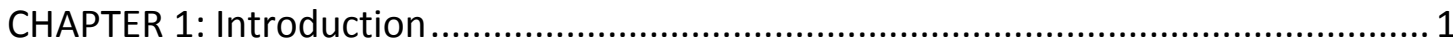

CHAPTER 2: Natural History of Habronattus oregonensis .......................................... 3

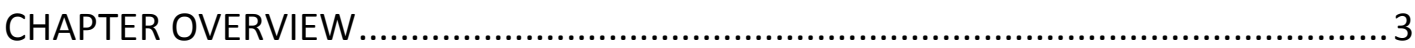

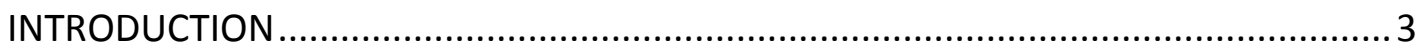

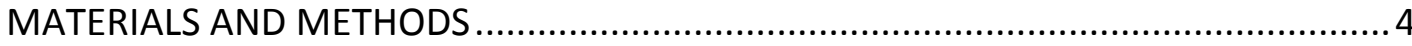

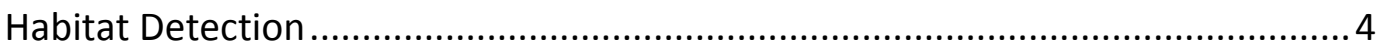

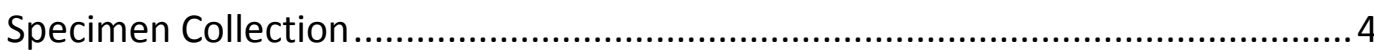

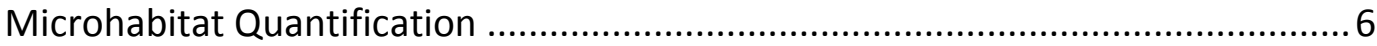

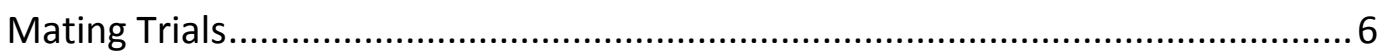

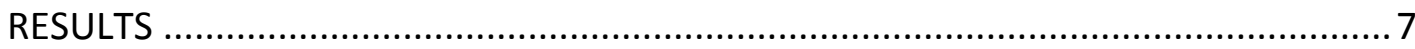

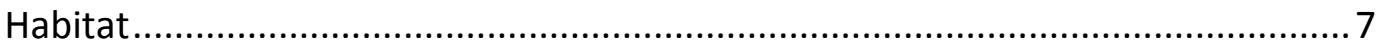

Weather .................................................................................................... 12

General Description of Color Differences between Sexes and Morphs .............. 13

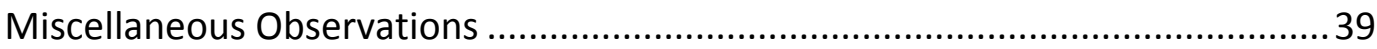

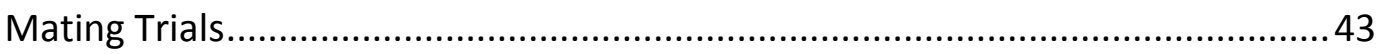

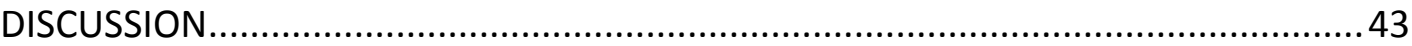

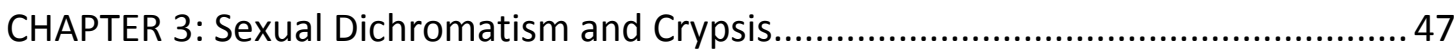

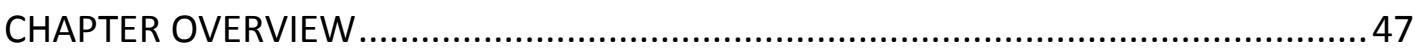

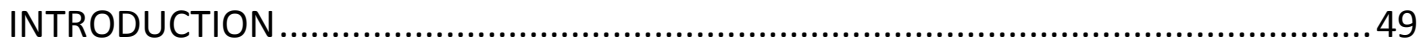

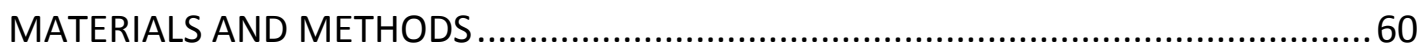

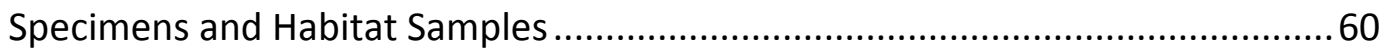

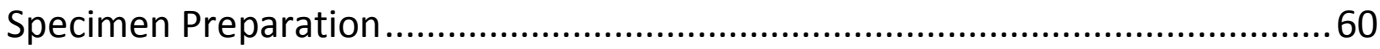

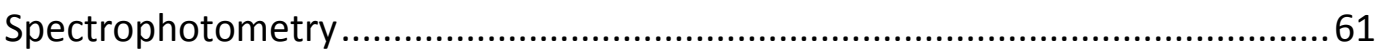

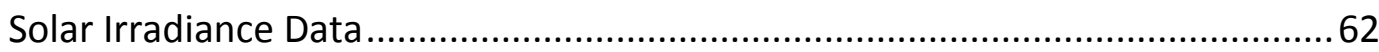

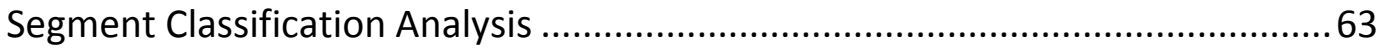

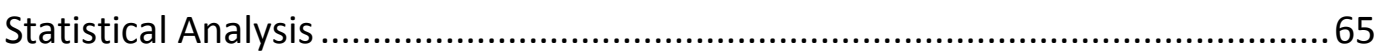

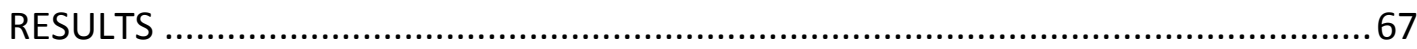

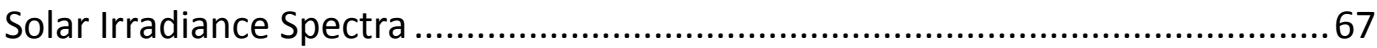

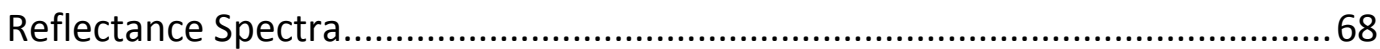

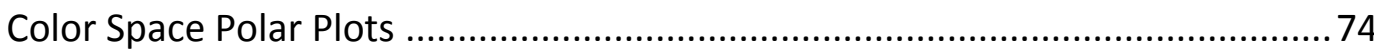

Color Space Polar Plots: Habitat Data ............................................................... 84

Color Space Polar Plots: Gorge and Siskiyou Populations ................................. 84

Color Space Polar Plots: Mt. Hood and Tillamook Populations ........................... 85

Differences in Spider and Habitat Hue, Chroma, and Brightness ........................ 86

Differences in Hue, Chroma, and Brightness Contrast Values between Spiders

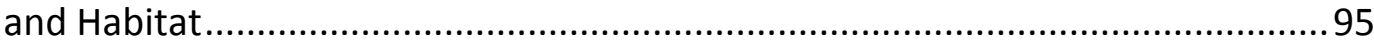

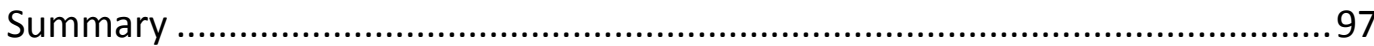

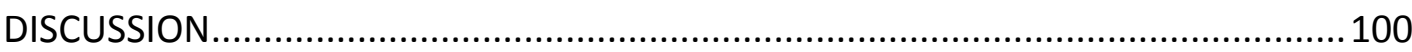

CHAPTER 4: Geographic Variation in Sexual Dimorphism and Male Sexual Ornaments Among Different Populations of Habronattus oregonensis .... 


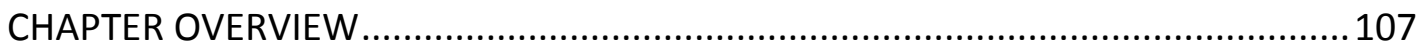

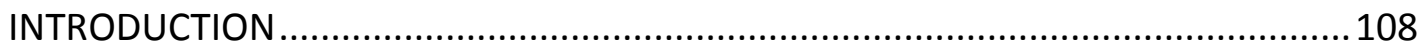

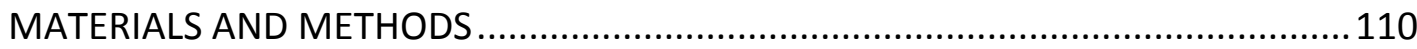

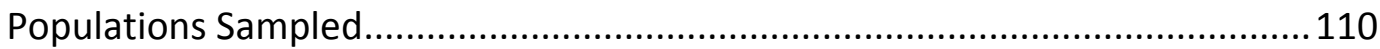

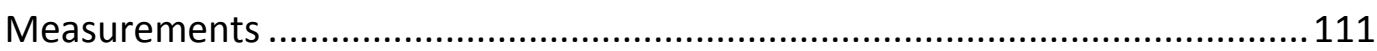

Morphometric Photography ................................................................ 112

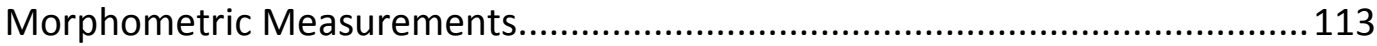

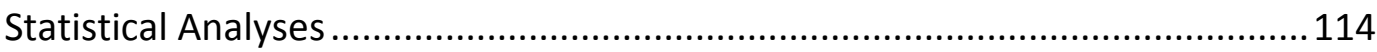

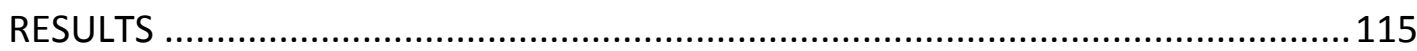

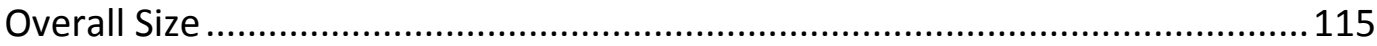

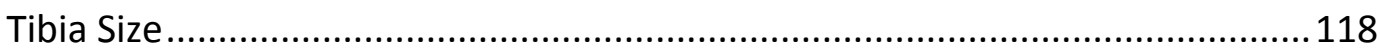

Male Tibia Size Relative to Overall Size....................................................... 122

Male Tibial Fringe Size Relative to Tibial Integument Size................................. 128

Classification of Individuals based on Morphology and Color........................... 131

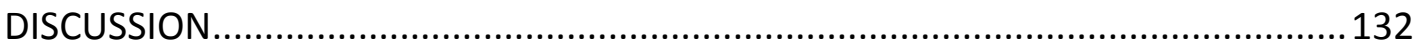

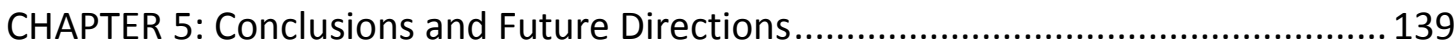

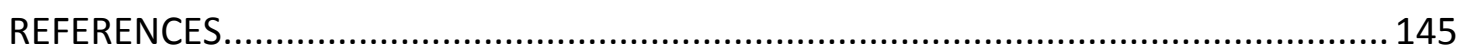

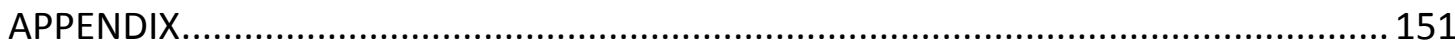


List of Figures

Figure 2.1. Gorge population habitat. ............................................................... 8

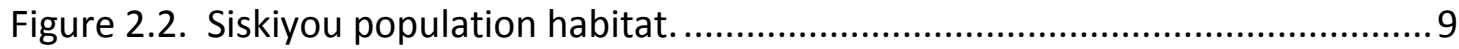

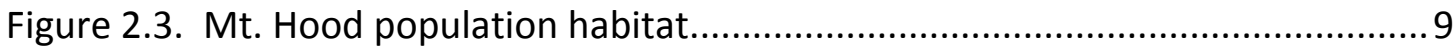

Figure 2.4. Tillamook population habitat. ............................................................. 10

Figure 2.5. Siskiyou site leaf litter habitat. ............................................................ 11

Figure 2.6. Number of $H$. oregonensis encountered by ambient air temperature...... 12

Figure 2.7. Male $H$. oregonensis Gorge population dorsal aspect (beige morph)..... 15

Figure 2.8. Male $H$. oregonensis Siskiyou population dorsal aspect (beige morph)...16

Figure 2.9. Female $H$. oregonensis Gorge population dorsal aspect (beige morph)...17

Figure 2.10. Female $H$. oregonensis Siskiyou population dorsal aspect (beige morph).

.. 18

Figure 2.11. Male H. oregonensis Gorge population anterior aspect (beige morph). 19 Figure 2.12. Male $H$. oregonensis Siskiyou population anterior aspect (beige morph).

20

Figure 2.13. Male $H$. oregonensis Gorge population view of palps (beige morph). ... 20 Figure 2.14. Male $H$. oregonensis Siskiyou population view of palps (beige morph). 21 Figure 2.15. Female H. oregonensis Gorge population anterior aspect (beige morph).

Figure 2.16. Female $H$. oregonensis Siskyiou population anterior aspect (beige morph). 23

Figure 2.17. Female $H$. oregonensis Gorge population view of palps (beige morph). 24 Figure 2.18. Female $H$. oregonensis Siskiyou population view of palps (beige morph).

Figure 2.19. Juvenile $H$. oregonensis Gorge population (beige morph)..................... 26

Figure 2.20. Juvenile $H$. oregonensis Siskiyou population (beige morph).................22

Figure 2.21. Male H. oregonensis Mt. Hood population dorsal aspect (bronze morph).

Figure 2.22. Male $H$. oregonensis Tillamook population dorsal aspect (bronze morph) 30

Figure 2.23. Female $H$. oregonensis Mt. Hood population dorsal aspect (bronze morph).

Figure 2.24. Female $H$. oregonensis Tillamook population dorsal aspect (bronze morph).

Figure 2.25. Male $H$. oregonensis Mt. Hood population anterior aspect (bronze morph)

Figure 2.26. Male $H$. oregonensis Tillamook population anterior aspect (bronze morph).

Figure 2.27. Male H. oregonensis Mt. Hood population view of palps (bronze morph). 
Figure 2.28. Male H. oregonensis Tillamook population view of palps (bronze morph).

Figure 2.29. Female $H$. oregonensis Mt. Hood population anterior aspect (bronze

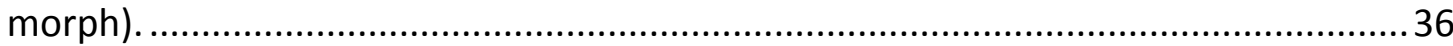

Figure 2.30. Female $H$. oregonensis Tillamook population anterior aspect (bronze

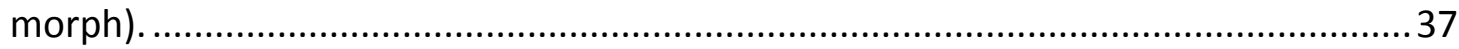

Figure 2.31. Female $H$. oregonensis Mt. Hood population view of palps (bronze morph).

Figure 2.32. Female $H$. oregonensis Tillamook population view of palps (bronze

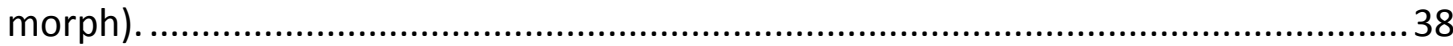

Figure 2.33. Juvenile $H$. oregonensis Tillamook population (bronze morph). ............ 39

Figure 2.34. Number of $H$. oregonensis encountered by time of day....................... 41

Figure 2.35. Number of $H$. oregonensis encountered by time of day, grouped by sex and developmental stage.

Figure 2.36. $H$. oregonensis and $H$. hirsutus conflict. ........................................... 42

Figure 2.37. Louse attached to posterior margin of cephalothorax of juvenile $H$.

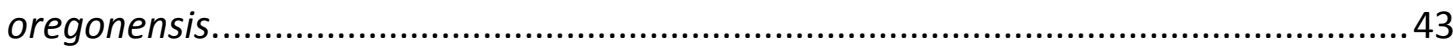

Figure 3.1. Spectral curves of two brightness levels of the same hue ("blue").........50

Figure 3.2. Spectral curves of two different chroma with the same peak ("blue")....50

Figure 3.3. Solar irradiance spectra...................................................................... 68

Figure 3.4. Gorge population reflectance: beige morph face...............................69

Figure 3.5. Mt. Hood population reflectance: bronze morph face............................ 70

Figure 3.6. Gorge population reflectance: beige morph cephalothorax................... 70

Figure 3.7. Mt. Hood population reflectance: bronze morph cephalothorax..............71

Figure 3.8. Gorge population reflectance: beige morph abdomen. .......................... 71

Figure 3.9. Mt. Hood population reflectance: bronze morph abdomen. .................. 72

Figure 3.10. Gorge population reflectance: beige morph ventrum........................ 72

Figure 3.11. Mt. Hood population reflectance: bronze morph ventrum................. 73

Figure 3.12. Gorge population habitat reflectance.............................................. 73

Figure 3.13. Mt. Hood population habitat reflectance. ......................................... 74

Figure 3.14. Gorge population, male hue and chroma color space polar plot:

reflectance data combined with solar irradiance data. ............................................ 76

Figure 3.15. Siskiyou population, male hue and chroma color space polar plot: reflectance data combined with solar irradiance data. ......................................... 77

Figure 3.16. Gorge population, female hue and chroma color space polar plot: reflectance data combined with solar irradiance data. ........................................... 78 Figure 3.17. Siskiyou population, female hue and chroma color space polar plot: reflectance data combined with solar irradiance data. ............................................. 79 Figure 3.18. Mt. Hood population, male hue and chroma color space polar plot: reflectance data combined with solar irradiance data. 80 
Figure 3.19. Tillamook population, male hue and chroma color space polar plot: reflectance data combined with solar irradiance data.

Figure 3.20. Mt. Hood population, female hue and chroma color space polar plot: reflectance data combined with solar irradiance data. 82

Figure 3.21. Tillamook population, female hue and chroma color space polar plot: reflectance data combined with solar irradiance data.

Figure 4.1. Mean carapace width of male spiders.

Figure 4.2. Mean carapace width of male and female spiders. ............................. 118

Figure 4.3. Mean "apparent" tibia area of male spiders grouped by population.....119

Figure 4.4. Mean "actual" tibia area of male and female spiders grouped by morph.

Figure 4.5. Mean "apparent" tibia area of male spiders grouped by morph.

Figure 4.6. Area of male tibia including setae fringe compared to carapace size, sorted by population, including trendlines.

Figure 4.7. Area of male tibia not including setae fringe compared to carapace size, sorted by population, including trendlines.

Figure 4.8. Area of male tibia (setae fringe only) compared to tibia area (integument only, not including setae), sorted by population, including trendlines. 
List of Tables

Table 2.1. Elevation range (meters above sea level) of spider populations.

Table 2.2. Occurrence of spiders on substrate types, sorted by population.

Table 2.3. Microhabitat components of the $20 \mathrm{~cm}$ radius quadrat around the point of

first detection of each spider, sorted by population.

Table 2.4. Mean temperature by population at which spiders were collected.

Table 2.5. Adjusted p-values from Tukey's HSD test for pairwise comparisons of mean temperatures of all four spider populations.

Table 3.1. Student's $t$-test comparing raw hue values of Gorge and Siskiyou habitat reflectance spectra with all Gorge and Siskiyou male and female body regions.

Table 3.2. Student's $t$-test comparing raw chroma values of Gorge and Siskiyou habitat reflectance spectra with all Gorge and Siskiyou male and female body regions.

Table 3.3. Student's $t$-test comparing raw brightness values of Gorge and Siskiyou habitat reflectance spectra with all Gorge and Siskiyou male and female body regions.

Table 3.4. Student's $t$-test comparing raw hue values of Mt. Hood and Tillamook habitat reflectance spectra with all Gorge and Siskiyou male and female body regions.

Table 3.5. Student's t-test comparing raw chroma values of Mt. Hood and Tillamook habitat reflectance spectra with all Gorge and Siskiyou male and female body regions.

Table 3.6. Student's $t$-test comparing raw brightness values of Mt. Hood and Tillamook habitat reflectance spectra with all Gorge and Siskiyou male and female body regions.

Table 3.7. Student's $t$-test pairwise comparison of raw hue values of Mt. Hood and Tillamook male and female face reflectance spectra.

Table 3.8. Student's $t$-test pairwise comparison of raw chroma values of Mt. Hood and Tillamook male and female face reflectance spectra.

Table 3.9. Student's $t$-test pairwise comparison of raw brightness values of Mt. Hood and Tillamook male and female face reflectance spectra. 93

Table 3.10. Standard Deviation of raw color values of all spider reflectance spectra sorted by population, sex, and body region as well as habitat reflectance spectra grouped by population.

Table 3.11. Contrast values of females of a single population versus the average of all (male and female) habitat reflectance spectra from their own population compared against the contrast values of males of the same population versus the average of all (male and female) habitat reflectance spectra from their own population................96 Table 3.12. Summary of main results, Gorge population..........................................98

Table 3.13. Summary of main results, Siskiyou population. ................................... 98 
Table 3.14. Summary of main results, Mt. Hood population.................................. 99

Table 3.15. Summary of main results, Tillamook population....................................99

Table 4.1. Sample sizes for morphometric analyses. .........................................110

Table 4.2. Adjusted p-values from Tukey's HSD test for pairwise comparisons of all

four spider populations for four measures of overall male spider size. ...................115

Table 4.3. Adjusted p-values from Tukey's HSD test for pairwise comparisons of both male and females spiders grouped by morph for four measures of overall spider size.

Table 4.4. Adjusted p-values from Tukey's HSD test for pairwise comparisons of all

four spider populations for five measures of male tibia size.................................. 119

Table 4.5. Adjusted p-values from Tukey's HSD test for pairwise comparisons of both

male and females spiders grouped by morph for five measures of spider tibia size.121 
CHAPTER 1: Introduction

Sexual selection has had a substantial role in the phenotypic evolution of many animals (Andersson, 1994). Males of polygynous species tend to be under the greatest selective pressure for the evolution of sexual display traits, oftentimes resulting in phenotypes that are maladaptive in circumstances other than courtship (Andersson, 1994; Kirkpatrick, 1987). Instances such as these, where a phenotype's characterization as adaptive or maladaptive is context-dependent, are ideal scenarios for investigating the interactions of multiple selective forces with distinct trajectories directing the evolution of the same trait. Quantitative studies on phenotypic characters influenced by both sexual selection and natural selection provide insight into how organisms balance selective pressures from their environment and from potential mates, and more broadly how multiple selective forces result in the evolution of compromises. One system that presents the opportunity to separate these effects is the interplay between selection for cryptic coloration and selection for sexual coloration of sexually dichromatic organisms, due to the visually apparent and quantifiable nature of the variables under scrutiny. It has been shown that the degree to which these selective forces interact in the determination of organism coloration is substantial (Andersson, 1994; Endler, 1983; Stuart-Fox et al., 2004); natural selection, especially as it relates to the genesis and maintenance of crypsis, tends to be a limiting factor in the emergence of sexual ornaments and dichromatism (Stuart-Fox and Ord, 2004). 
In this study I first present natural history information on the little-known species of jumping spider, Habronattus oregonensis (chapter 2). In chapter 3 I examine the relationship between the coloration of $H$. oregonensis and its background substrate color, quantifying how the degree of animal/background color contrast differs between males and females, as well as among different populations of the same species. In chapter $4 \mathrm{I}$ address size dimorphisms among populations of this species, with emphasis on differences between male sexual ornaments, and in chapter 5 I finish with a discussion of the overall trends of this study and their implications, as well as some recommendations for future study directions. 
CHAPTER 2: Natural History of Habronattus oregonensis

\section{CHAPTER OVERVIEW}

Here I present basic natural history information of the jumping spider Habronattus oregonensis. For this study, populations were collected from four sites in Oregon and Washington. I made detailed observations on the date, time, location, temperature, weather conditions, habitat type, and behavior of each spider encountered. I most often found spiders from all locations on exposed rocky slopes with minimal vegetation nearby on still, sunny days. Spiders were active throughout the daylight hours. While air temperature values ranged between 17 and $34^{\circ} \mathrm{C}$, substrate temperature seems to be a more accurate herald of spider activity; I nearly always found spiders on warm substrates, rarely encountering them on cool or hot surfaces.

\section{INTRODUCTION}

Little is known about the natural history of $H$. oregonensis. Griswold (1987) published the most thorough account on the species and its distribution, noting its presence throughout much of the western United States. Griswold also described two broad color morphs, generally matching the beige and bronze morphs discussed here. However, Griswold's account of the species and the few surveys that have come after it (Crawford, 1988; Paquin et al., 2010) were based on only a handful of records; more recent collections suggest that there may be a broader distribution of 
the species, and a greater variation in its dichromatism and dimorphism than has hitherto been described (S. Masta, pers. comm.). Here I present data on the spider's basic macro and microhabitat preferences in four locations in the Pacific Northwest. When collecting the spiders for phenotypic analyses, I took the opportunity to gather additional natural history data on the species. Spiders were collected from multiple sites in Oregon and a single site in Washington.

\section{MATERIALS AND METHODS}

Habitat Detection

$H$. oregonensis has an expansive range in the North American continent, with collection sites ranging from Vancouver, British Columbia and Alberta through New Mexico and southern Arizona (S. Masta, pers. comm.; Griswold, 1987; Paquin et al., 2010; Peckham and Peckham, 1909). For this study, however, I focus only on populations in Oregon and the southernmost reaches of Washington. Due to the patchy distribution of $H$. oregonensis populations, search areas within the overall region of study were largely determined haphazardly, with more specific areas decided upon by seeking out locations deemed likely to have $H$. oregonensis habitat using topographic maps.

Specimen Collection 
Spiders were collected from four different sites in Oregon and Washington between 16 June 2009 and 21 July 2009; several other populations were located, though too few individuals were collected to be of use to this study. No permits were necessary for collection (personal communications, United States Forest Service, United States Fish and Wildlife Service, Oregon Department of Fish and Wildlife). The four populations addressed in this study are the following:

- Tillamook State Forest (Tillamook population)

- Columbia River Gorge National Scenic Area (Gorge population)

- Mount Hood National Forest (Mt. Hood population)

- Rogue River-Siskiyou National Forest (Siskiyou population)

Upon collection of each individual I recorded the following data: date, time, location (latitude/longitude coordinates and elevation using a GPS unit), air temperature (using an analogue thermometer, approximately 1.5 meters above ground level), substrate temperature (qualitative estimate by touch), weather conditions, habitat type, the degree of sun exposure on the patch of habitat, whether there were any other $H$. oregonensis in the vicinity, the presence of potential predators, the sex of the individual, and what type of activity the spider was engaged in when first encountered. While the vast majority of individuals encountered were adults, some juveniles were found; these were brought back to the lab to be reared to adulthood. I euthanized and preserved specimens by freezing at $-80^{\circ} \mathrm{C}$. 
Microhabitat Quantification

In addition to collecting the spiders, I gathered representative fragments of the habitat upon which they were initially encountered, out to a radius of twenty centimeters. I took a photograph of this quadrat with the precise center of the frame indicating the location at which the spider was first sighted. I used a tripod to standardize the distance from the camera to the substrate and to orient the camera perpendicularly to the substrate; I placed a small ruler at the edge of the frame to indicate scale. I took periodic photographs of the surrounding area for documentation of the habitat at a larger scale.

Mating Trials

I conducted mating trials with one of the females collected from the Gorge population; I presented her with four different males (one per trial), two from the Siskiyou population and two from the Tillamook population. I modeled trials after those described in Masta and Maddison (2002). Only virgin females are generally receptive towards mating advances (S. Masta, pers. comm.); this limited the mating trials to the only juvenile female reared to adulthood in captivity at a time when live males were available. I fed all individuals involved in mating trials the previous day in order to minimize the temptation of predation upon rejected suitors. The mating arena consisted of a blank white sheet of paper substrate enclosed by a transparent plastic cylinder (diameter: $17 \mathrm{~cm}$, height: $11 \mathrm{~cm}$ ) open at the top and illuminated from 
above. I added the female to the mating arena first and allowed to acclimate to her surroundings (approximately two minutes, or until she was calmly situated on the floor of the arena), at which point I added the male. Fifteen minutes of rejection or the initiation of aggressive advances terminated the trial. I videotaped all trials.

\section{RESULTS}

Habitat

I found spiders as low as 206 meters above sea level and as high as 1129 meters (Table 2.1). There was no discernable pattern in elevational distribution if populations are grouped by color morph: beige morphs were found at the low and the high extremes; bronze morphs were found from $597 \mathrm{~m}$ to $1092 \mathrm{~m}$ a.s.I. In nearly all instances, I found the spiders in the open in full sun or near the edge of sparse shade provided by a small plant or shrub. I found nearly all spiders on steep slopes (approximately $45^{\circ}$ ); the few that weren't were immediately adjacent to such slopes. I rarely found the spiders in areas completely devoid of vegetation; instead they preferred extremely low-density herbaceous vegetation and/or the presence of a few seedlings or saplings (Figures 2.1 through 2.4). I found the vast majority of spiders on rocks; alternative substrates were dried deciduous leaves, twigs, or fragments of bark. Although preference for rock substrate was evident in all populations, there was a noticeable difference among the populations in secondary substrate preference, with Gorge and Siskiyou individuals occasionally being found on dried 
leaves and Mt. Hood and Tillamook individuals never being found on dried leaves. A chi-square analysis found these differences to be significant; $p$-value $=0.006$ (Table 2.2). Microhabitat components of the immediate vicinity $(20 \mathrm{~cm}$ radius around point of first detection) of the spiders were more varied, but still reflect a bias towards rocks (Table 2.3).

Table 2.1. Elevation range (meters above sea level) of spider populations.

\begin{tabular}{lcccc}
\hline \hline & \multicolumn{4}{c}{ Population } \\
\cline { 2 - 5 } & Gorge & Siskiyou & Mt. Hood & Tillamook \\
\hline Elevation range & $206-246$ & $567-1129$ & $904-970$ & $597-661$ \\
\hline
\end{tabular}

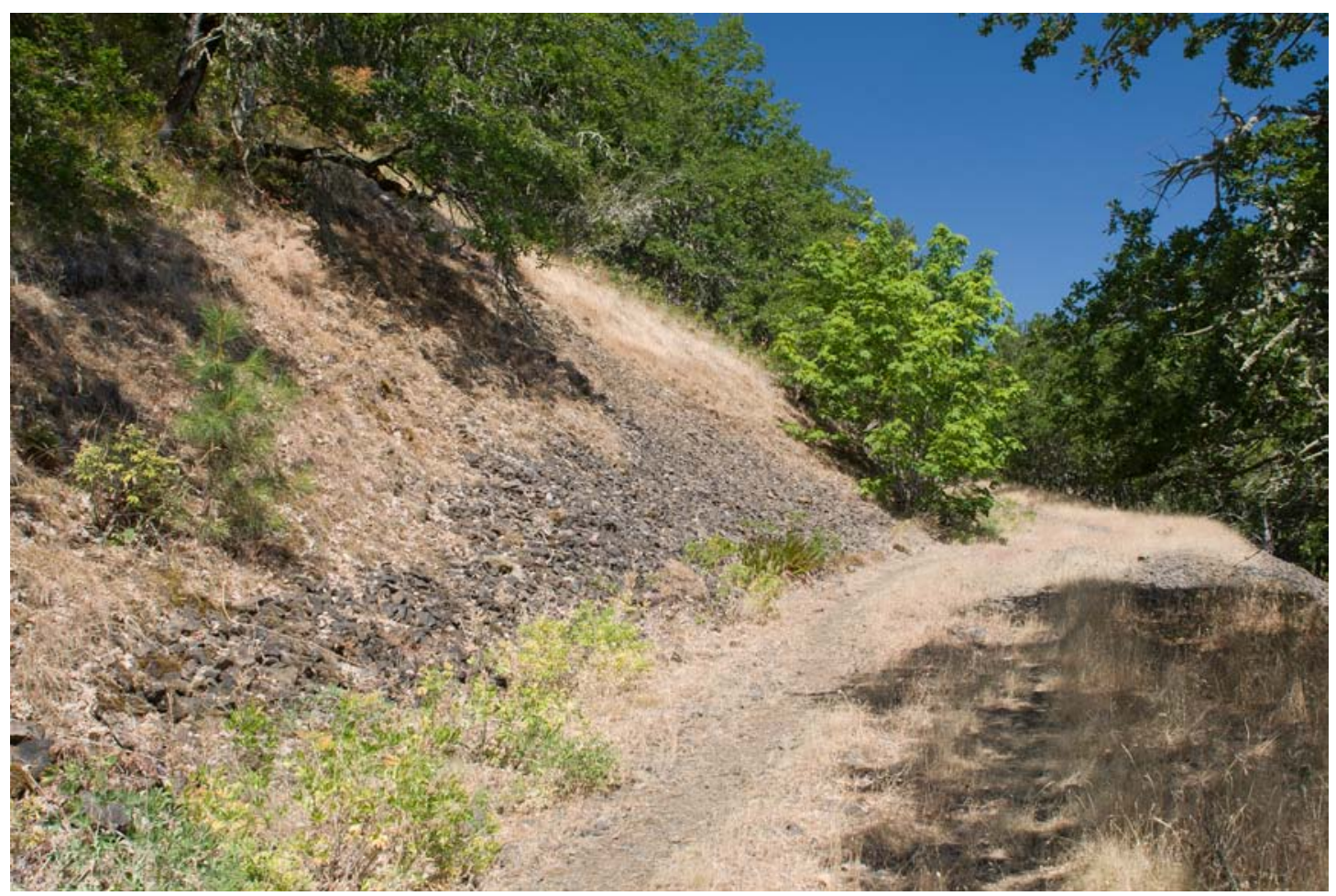

Figure 2.1. Gorge population habitat. 


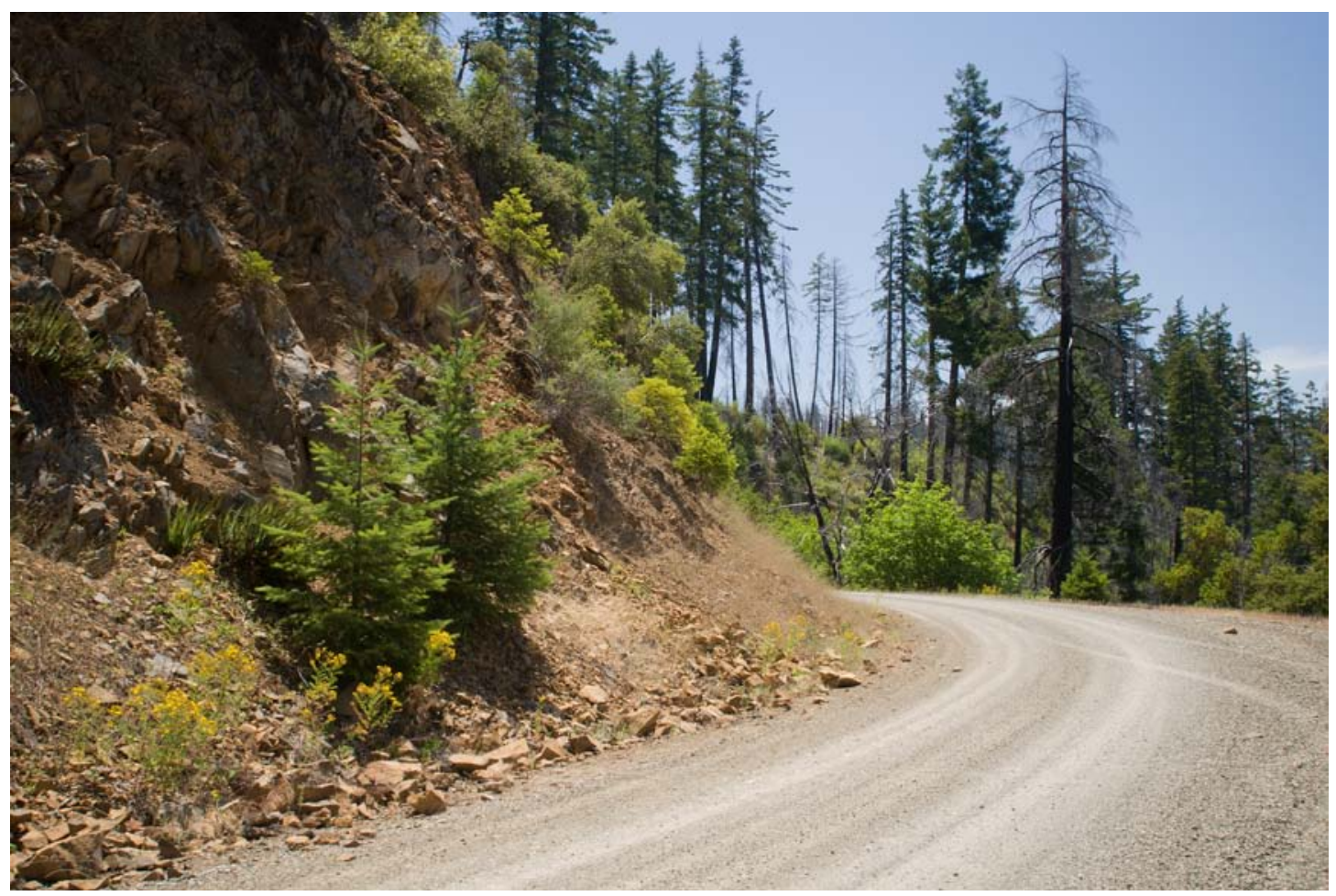

Figure 2.2. Siskiyou population habitat.

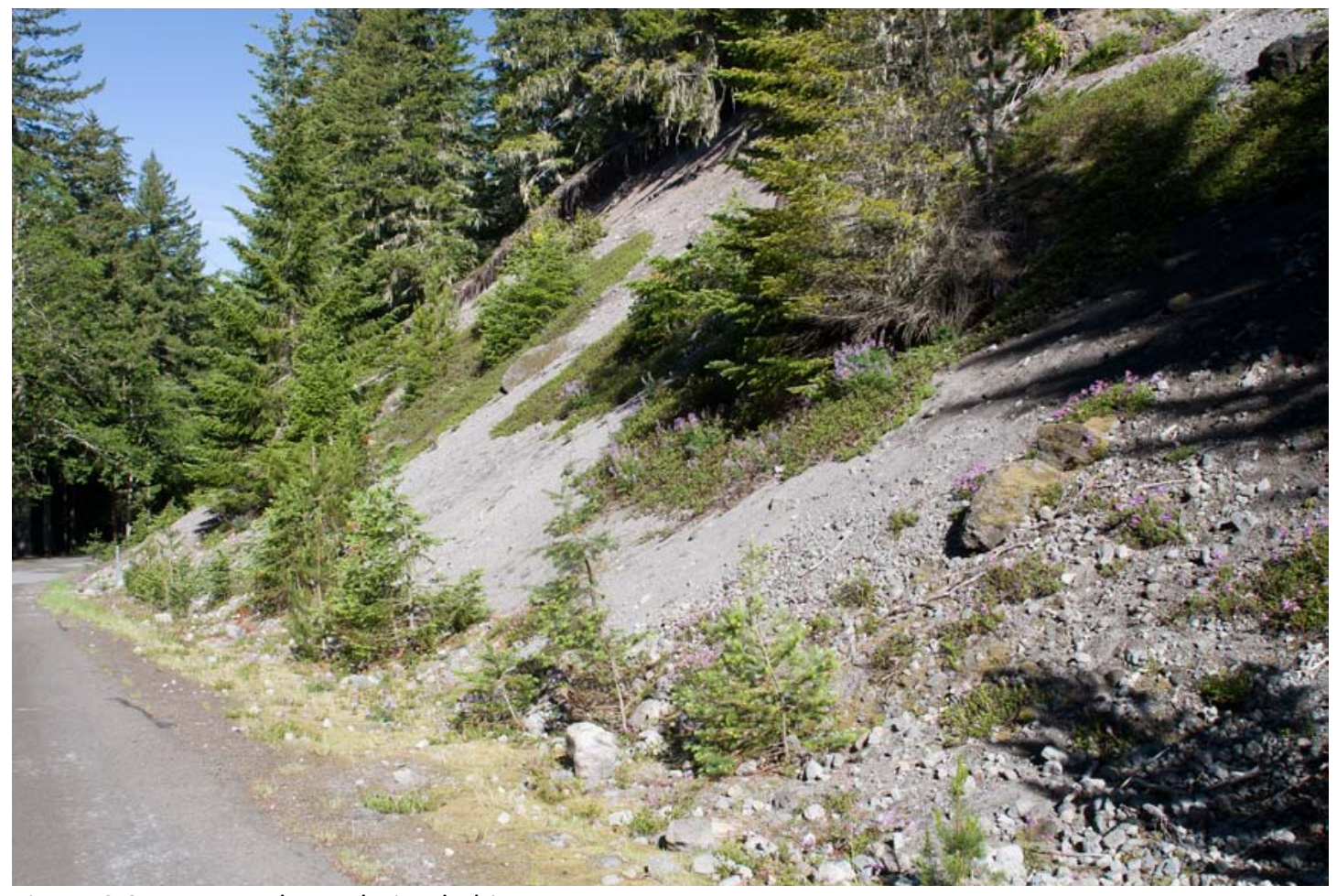

Figure 2.3. Mt. Hood population habitat. 


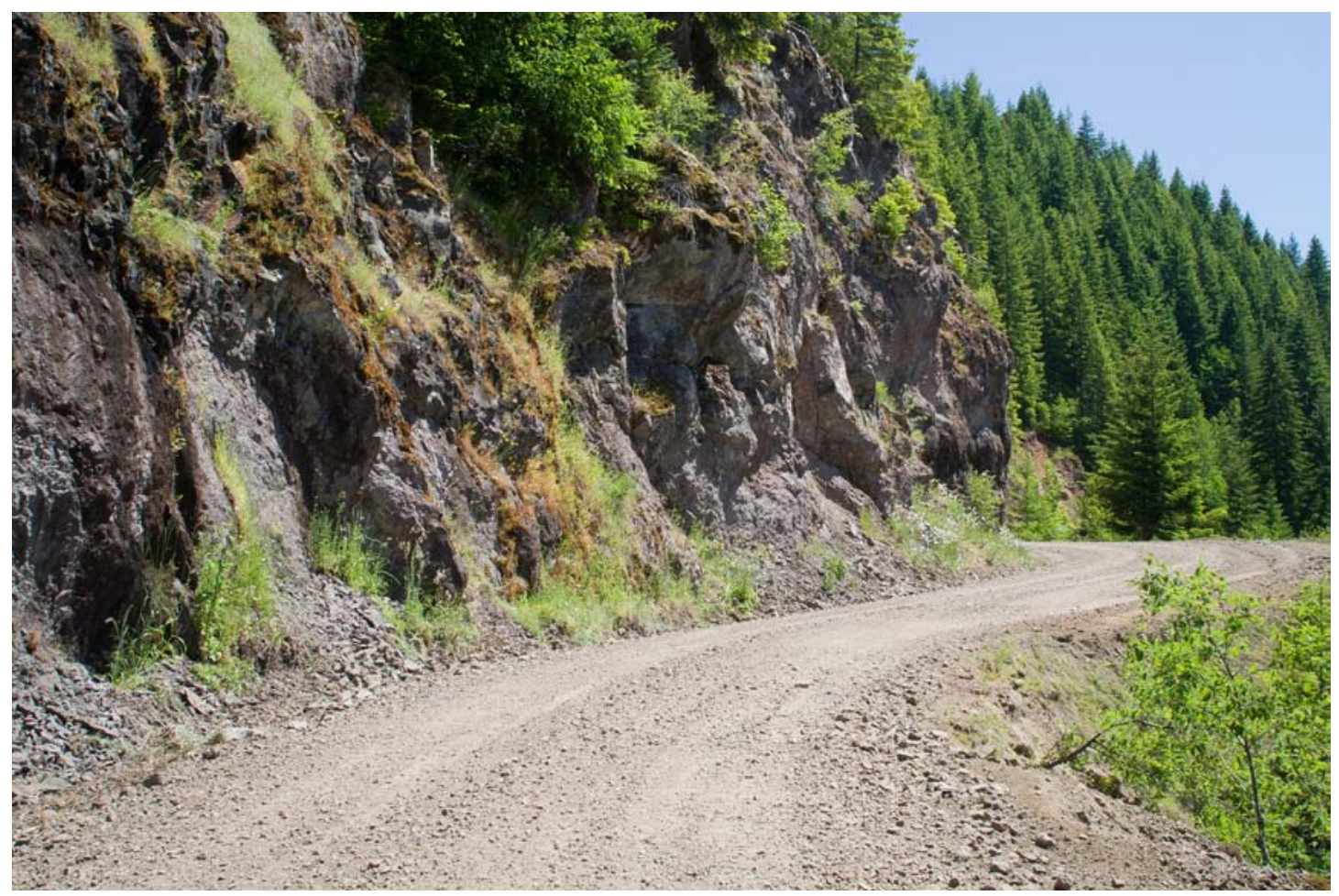

Figure 2.4. Tillamook population habitat.

Table 2.2. Occurrence of spiders on substrate types, sorted by population.

\begin{tabular}{lcccc}
\hline \hline & Gorge & Siskiyou & Mt. Hood & Tillamook \\
\hline Dry leaf & $32.14 \%$ & $16.67 \%$ & $0.00 \%$ & $0.00 \%$ \\
Twig/bark & $0.00 \%$ & $11.11 \%$ & $15.79 \%$ & $12.00 \%$ \\
Rock & $67.86 \%$ & $72.22 \%$ & $84.21 \%$ & $88.00 \%$ \\
\hline
\end{tabular}

Table 2.3. Microhabitat components of the $20 \mathrm{~cm}$ radius quadrat around the point of first detection of each spider, sorted by population.

\begin{tabular}{lcccc}
\hline \hline & Gorge & Siskiyou & Mt. Hood & Tillamook \\
\hline Rock & $85.71 \%$ & $83.33 \%$ & $100.00 \%$ & $100.00 \%$ \\
Dry leaf & $89.29 \%$ & $44.44 \%$ & $0.00 \%$ & $8.00 \%$ \\
Green leaf & $3.57 \%$ & $27.78 \%$ & $68.42 \%$ & $4.00 \%$ \\
Twig & $3.57 \%$ & $55.56 \%$ & $21.05 \%$ & $24.00 \%$ \\
Bark & $0.00 \%$ & $5.56 \%$ & $0.00 \%$ & $12.00 \%$ \\
Dry grass & $7.14 \%$ & $0.00 \%$ & $10.53 \%$ & $8.00 \%$ \\
Green grass & $3.57 \%$ & $0.00 \%$ & $0.00 \%$ & $4.00 \%$ \\
Lichen & $3.57 \%$ & $0.00 \%$ & $0.00 \%$ & $0.00 \%$
\end{tabular}




\begin{tabular}{lcccc} 
Soil & $0.00 \%$ & $11.11 \%$ & $21.05 \%$ & $4.00 \%$ \\
Pine cone & $0.00 \%$ & $5.56 \%$ & $26.32 \%$ & $4.00 \%$ \\
Detritus & $3.57 \%$ & $5.56 \%$ & $0.00 \%$ & $0.00 \%$ \\
Acorn & $0.00 \%$ & $11.11 \%$ & $0.00 \%$ & $0.00 \%$ \\
\hline
\end{tabular}

The site of the Siskiyou population was somewhat atypical compared to the rest of the sites sampled in this study, including the sites that were omitted from the analysis presented here. This habitat had more deciduous trees, including a large oak component. H. oregonensis at this site was associated with rocks and slopes as per the trend at all other sites, but additionally was found on leaf litter in areas where there was no dominant rocky component (even on leaf litter, the spiders were still only encountered on slopes) (Figure 2.5).

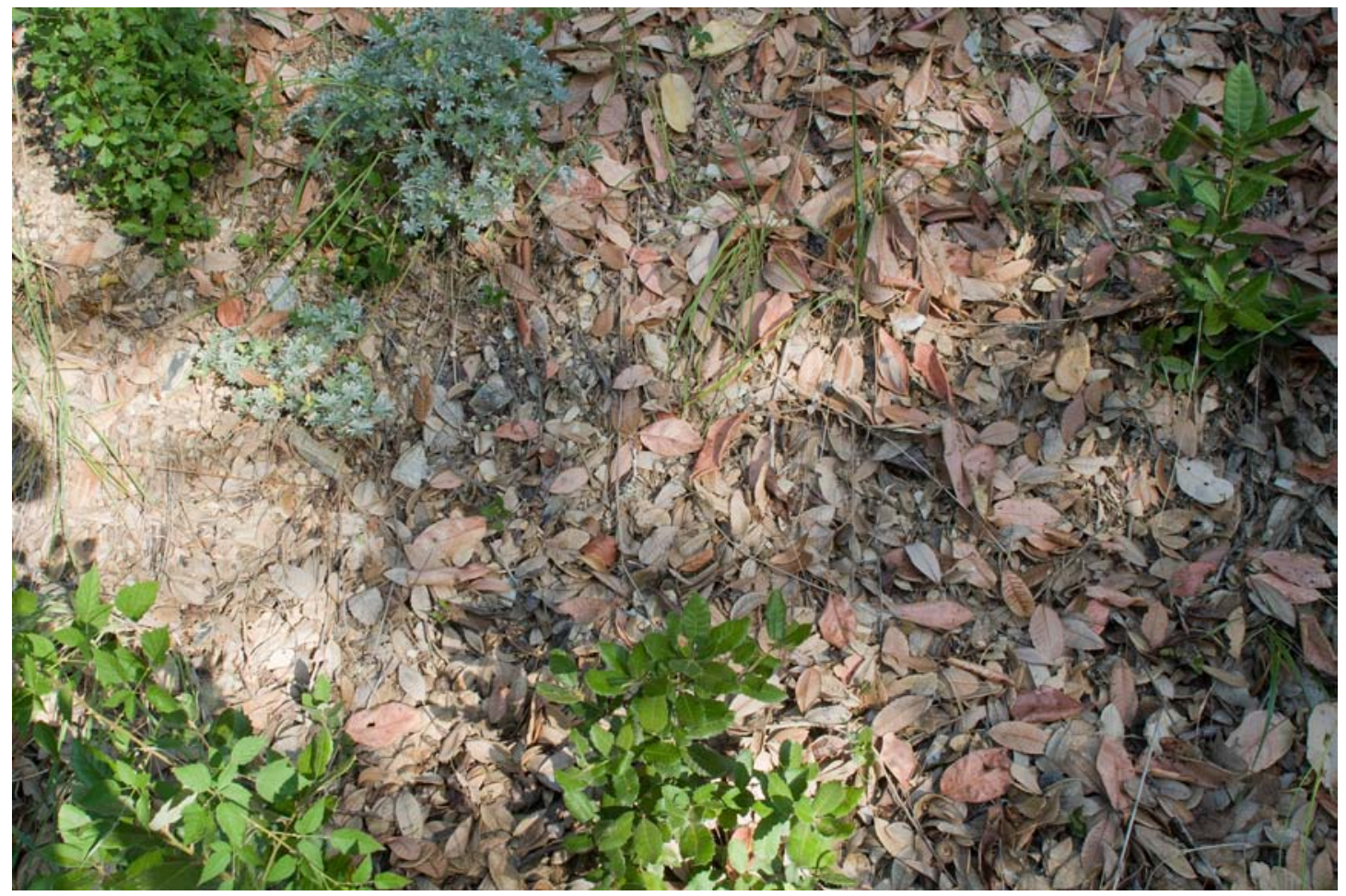

Figure 2.5. Siskiyou site leaf litter habitat. 
Weather

I found spiders in air temperatures ranging from 17 to $34^{\circ} \mathrm{C}$ (Figure 2.6). The mean temperatures at which I encountered spiders are displayed in Table 2.4. An ANOVA revealed a significant difference in the mean temperature at which I found individuals from the four target populations. A Tukey's HSD pairwise test between the mean temperatures of the populations identified the Mt. Hood population as significantly different from all other populations; no other populations were significantly different from each other (Table 2.5). I found all spiders in clear to mostly clear sunny conditions or with a slight haze present, with little to no breeze. I nearly always found spiders when the rocks were between ambient temperature and warm to touch; in rare instances were the rocks cool or very warm/hot.

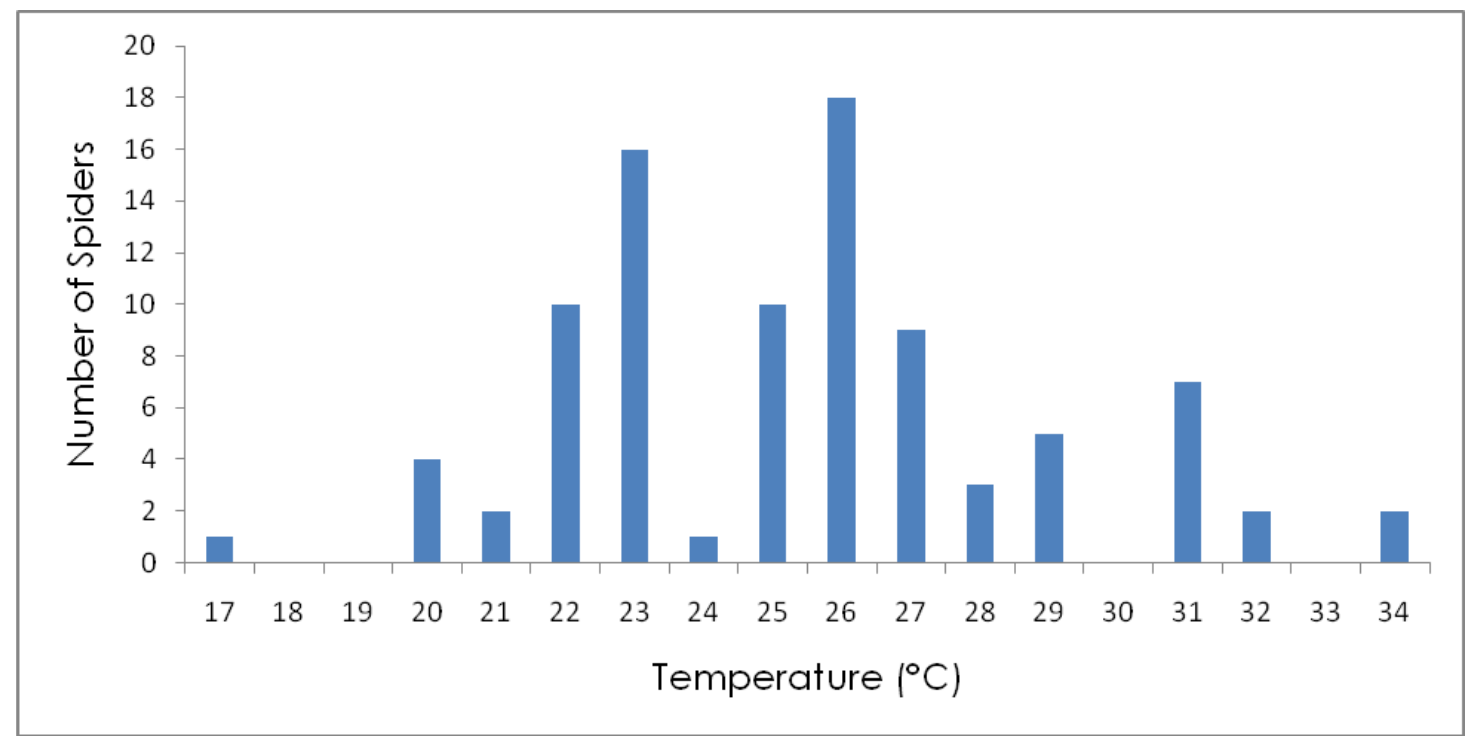

Figure 2.6. Number of $H$. oregonensis encountered by ambient air temperature. 
Table 2.4. Mean temperature by population at which spiders were collected.

\begin{tabular}{lc}
\hline \hline Population & Temperature $\left({ }^{\circ} \mathrm{C}\right)$ \\
\hline Gorge & 26.4 \\
Siskiyou & 25.9 \\
Mt. Hood & 21.6 \\
Tillamook & 26.4 \\
\hline
\end{tabular}

Table 2.5. Adjusted p-values from Tukey's HSD test for pairwise comparisons of mean temperatures of all four spider populations.

\begin{tabular}{lc}
\hline \hline Population Comparison & Adjusted p-value \\
\hline Mt. Hood - Gorge & 0.000 \\
Tillamook - Gorge & 1.000 \\
Siskiyou - Gorge & 0.923 \\
Tillamook - Mt. Hood & 0.000 \\
Siskiyou - Mt. Hood & 0.000 \\
Siskiyou - Tillamook & 0.909 \\
\hline
\end{tabular}

General Description of Color Differences between Sexes and Morphs

I found two distinct color morphs in this study: the beige morph and the bronze morph. I never found more than one morph in a population; differences in coloration between populations of the same morphs are subtle to nonexistent (see chapter 3 for a quantitative analysis of coloration). Here I present descriptions of the two morphs, with supporting photographs from each population.

The dorsal coloration of beige morph males ranges from nearly white on parts of the cephalothorax to rather dark on patches of the abdomen; abdominal setae are slightly glossy (Figures 2.7 and 2.8). Female dorsal setae are matte and generally uniform in color (Figures 2.9 and 2.10). Anteriorly, beige morph males have light and dark setae scattered over the face creating a high-contrast speckling; the anterior 
eyes are rimmed with light setae and the chelicerae are covered in orangish setae (Figures 2.11 and 2.12). The lower margin of the carapace has two parallel lines of white setae with a black line between terminating at the outer edges of the clypeus. The palps are dark with the lower third covered in orangish setae (Figures 2.13 and 2.14). Similar to that of the males, the eyes of beige morph females are rimmed with light setae and they have two lines of white setae fringing their carapace, though these lines are thinner and the topmost white line continues over the clypeus (Figures 2.15 and 2.16). The rest of the female face is nearly uniformly dark; their chelicerae have only a sparse scattering of setae, and their face is mostly black (with some of their light dorsal setae visible above their anterior eyes). Their palps have sparse fringing with mostly light-colored setae (Figures 2.17 and 2.18). Beige morph juveniles are very similar to females with respect to color; palp setae of juveniles tend to be just a little shorter with a little less light-colored setae than those of adult females (Figures 2.19 and 2.20). 


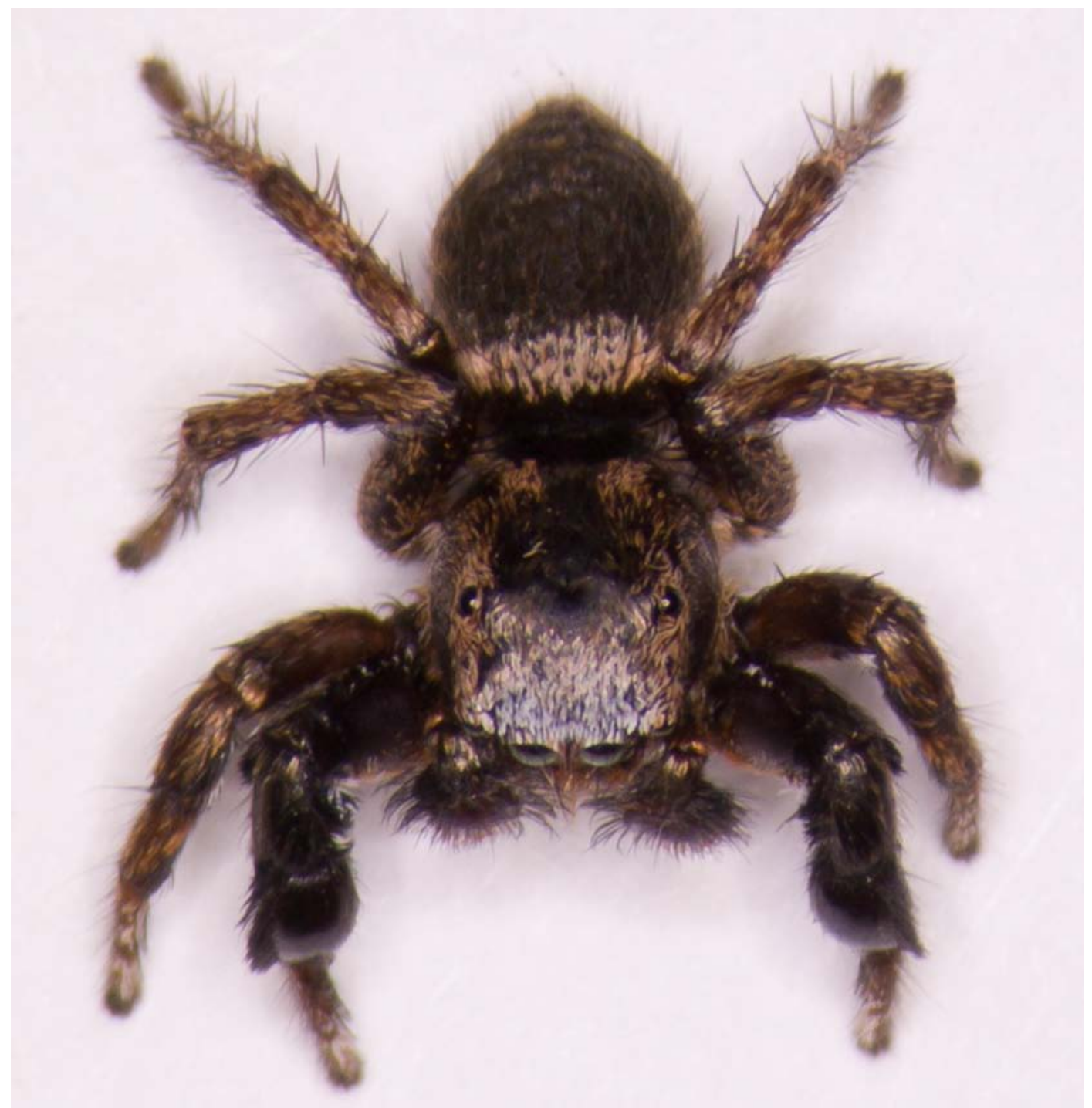

Figure 2.7. Male H. oregonensis Gorge population dorsal aspect (beige morph). Specimen ID\# JB002. 


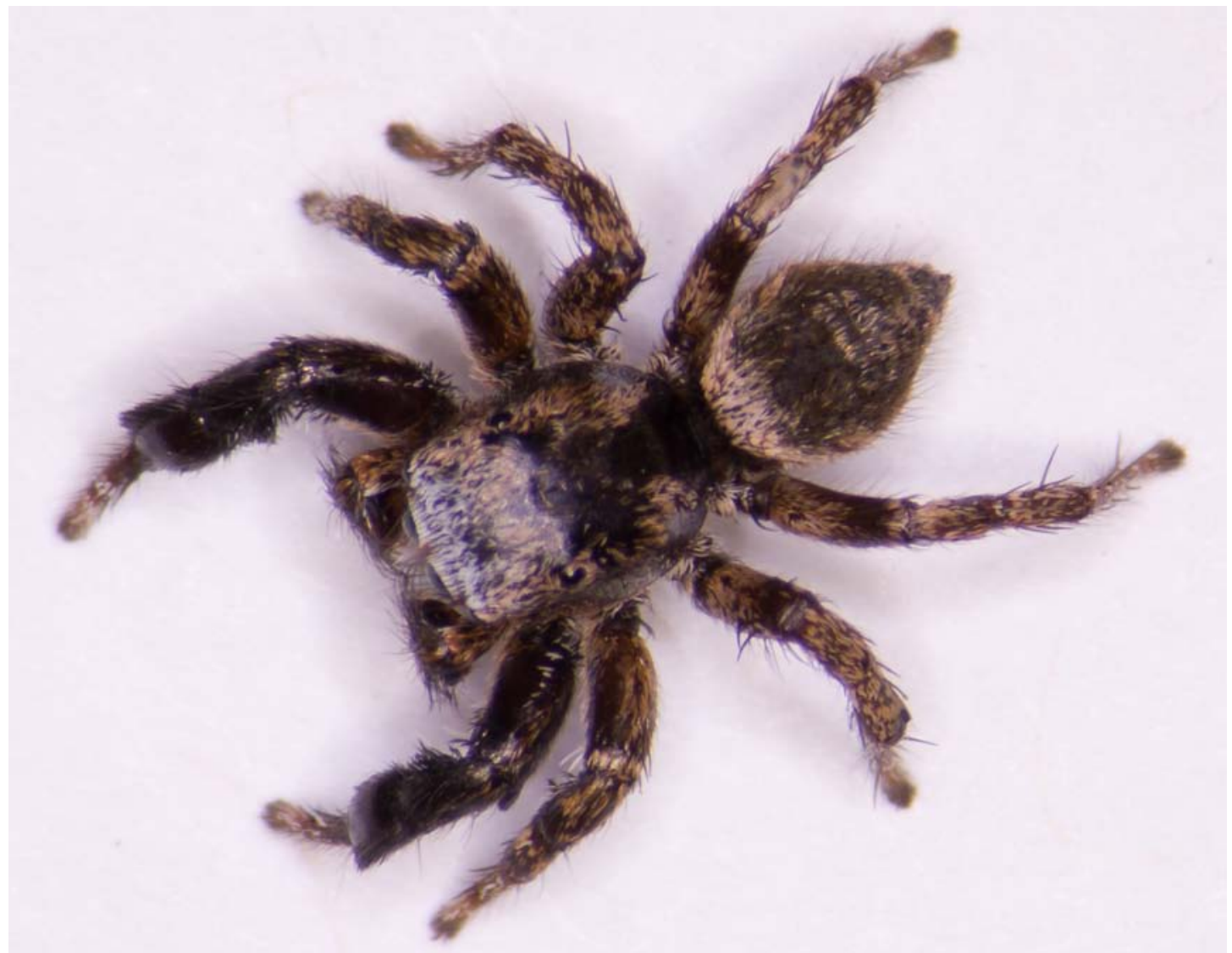

Figure 2.8. Male $H$. oregonensis Siskiyou population dorsal aspect (beige morph). Specimen ID\# JB088. 


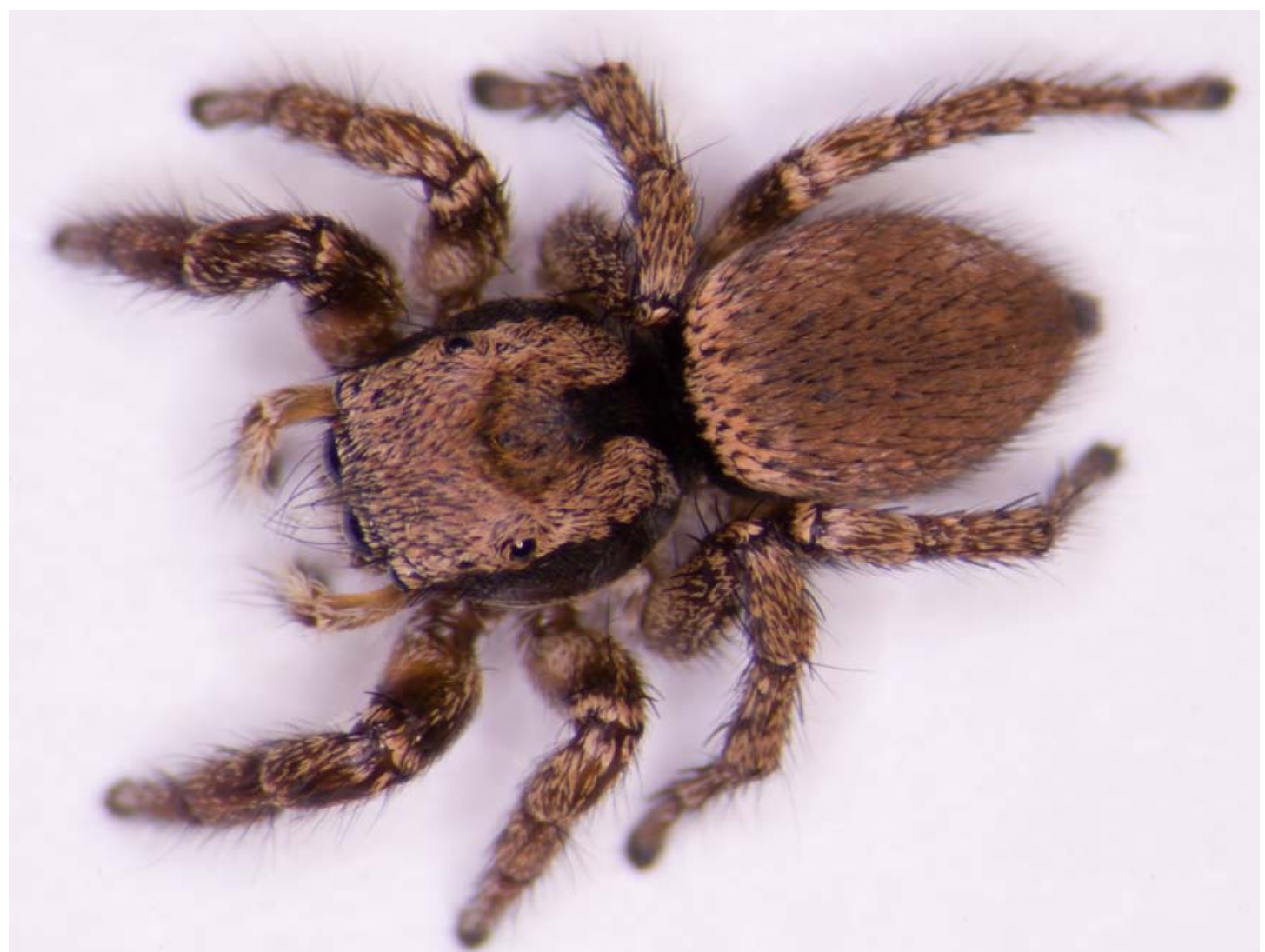

Figure 2.9. Female $H$. oregonensis Gorge population dorsal aspect (beige morph). Specimen ID\# JB005. 


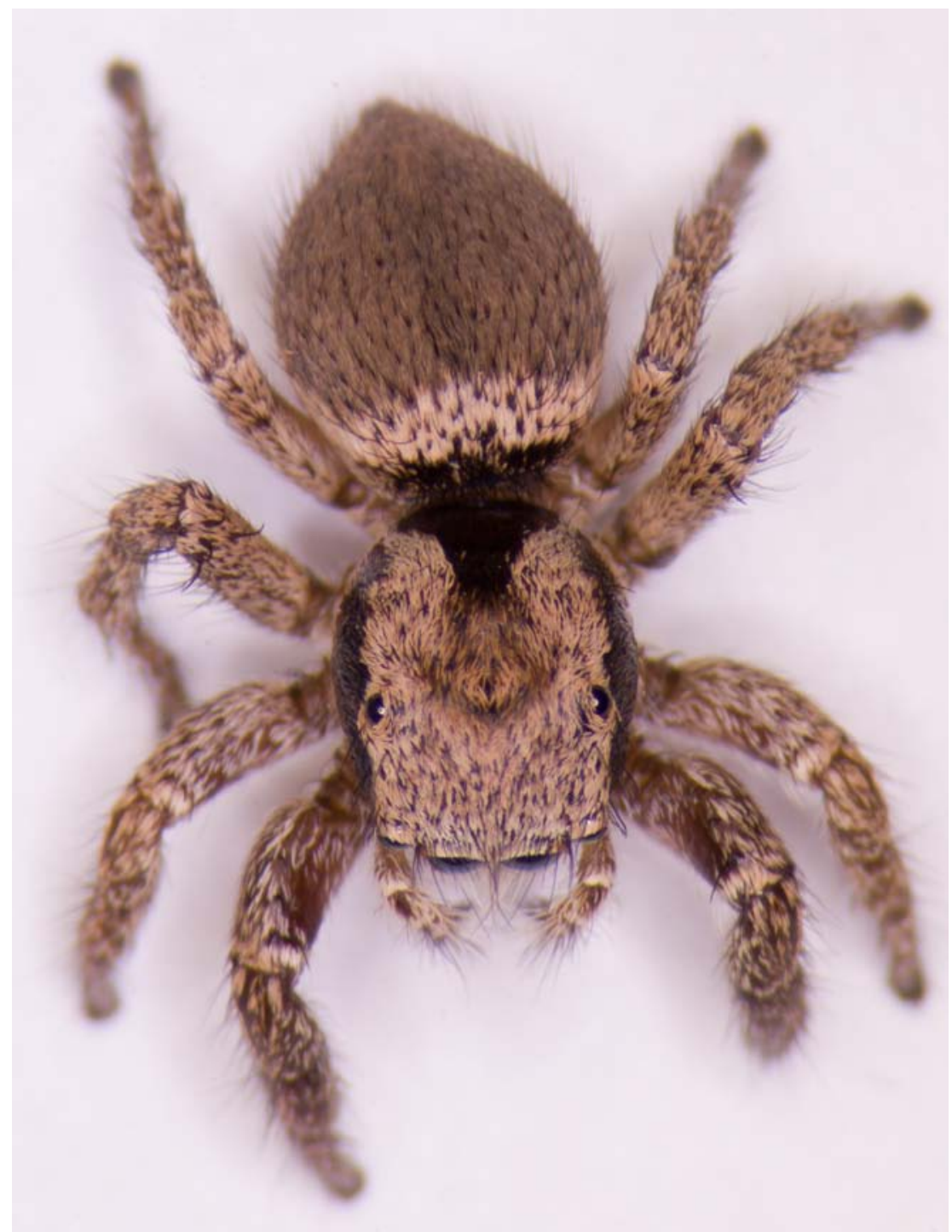

Figure 2.10. Female $H$. oregonensis Siskiyou population dorsal aspect (beige morph). Specimen ID\# JB080. 


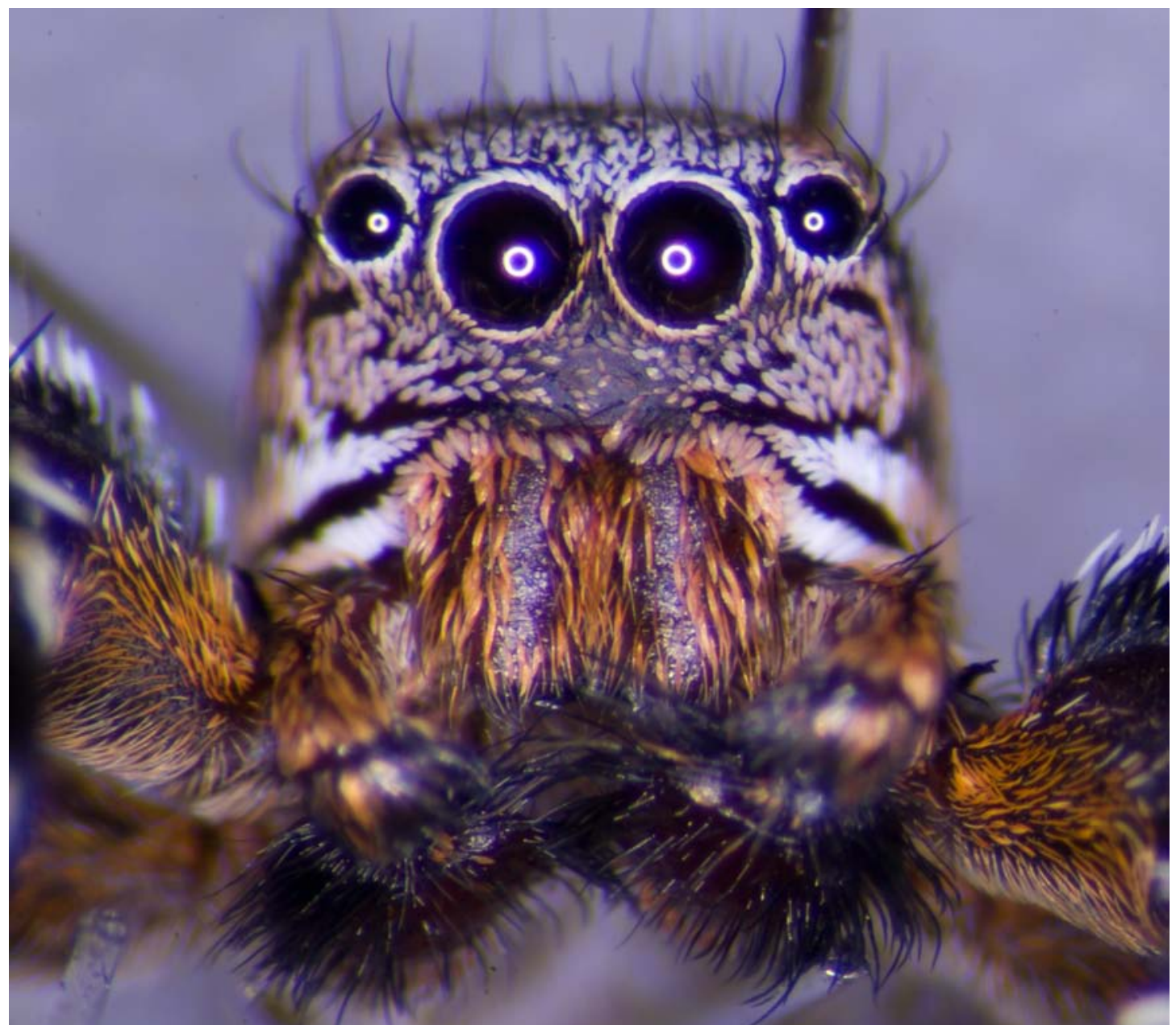

Figure 2.11. Male H. oregonensis Gorge population anterior aspect (beige morph). Specimen ID\# JB003. 


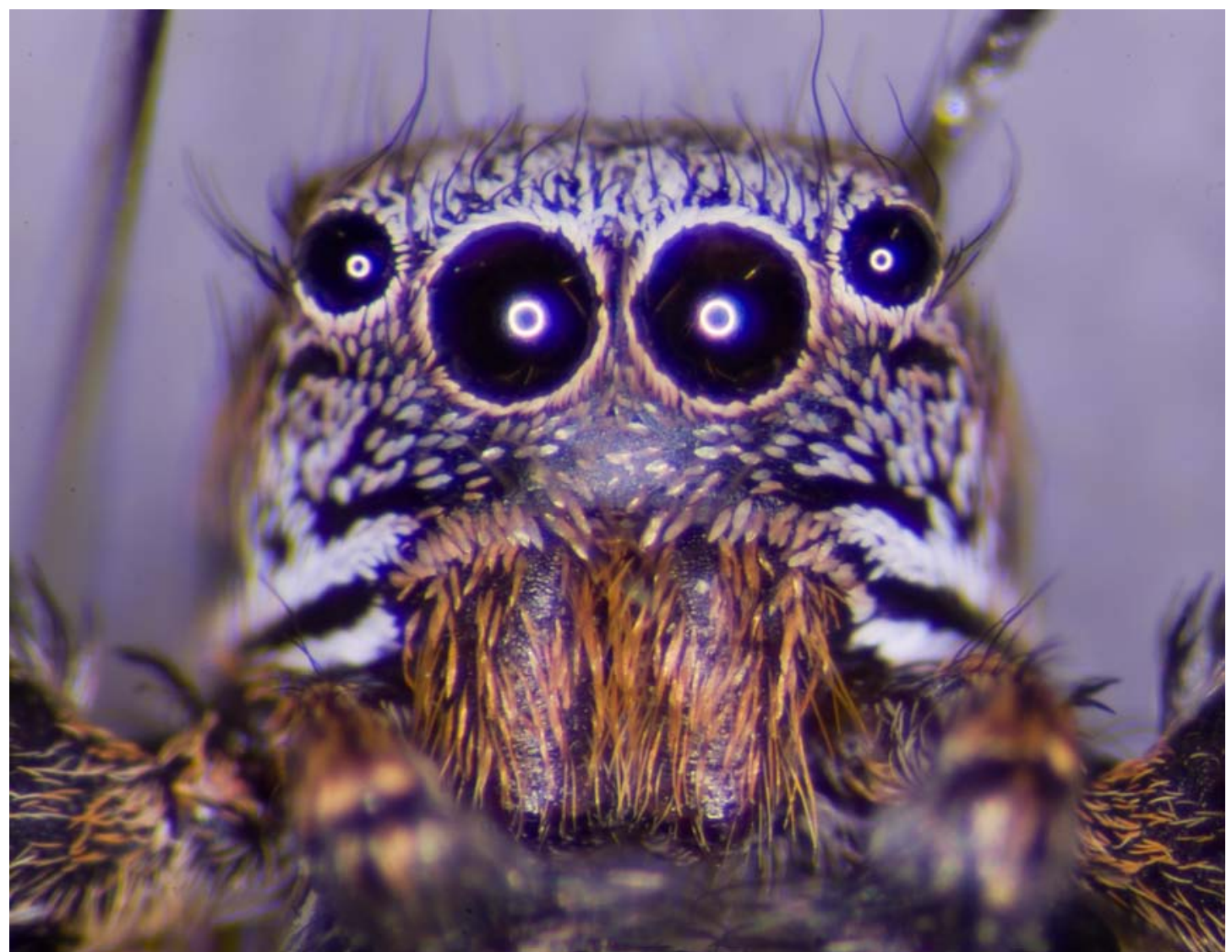

Figure 2.12. Male $H$. oregonensis Siskiyou population anterior aspect (beige morph). Specimen ID\# JB088.

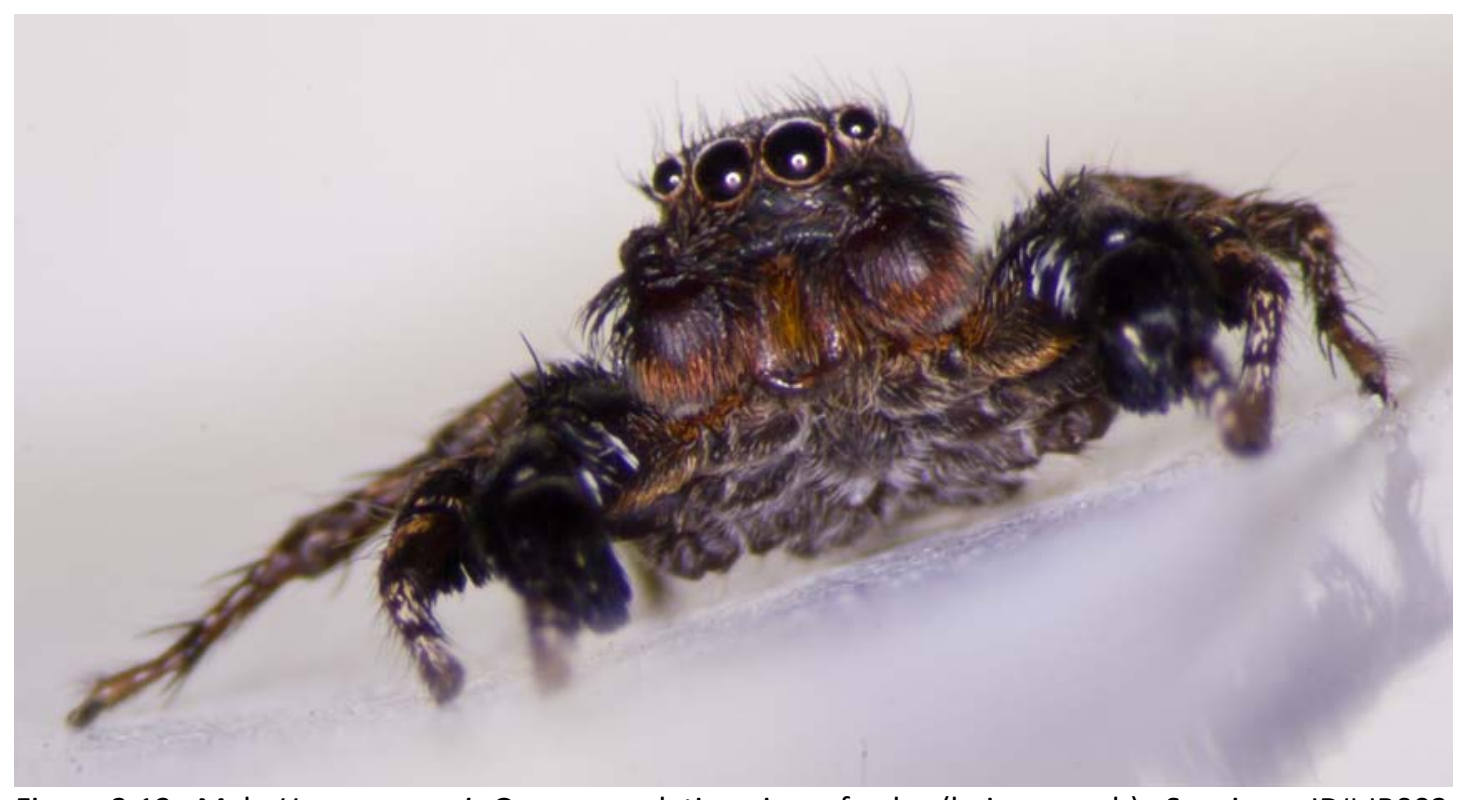

Figure 2.13. Male H. oregonensis Gorge population view of palps (beige morph). Specimen ID\# JB002. 


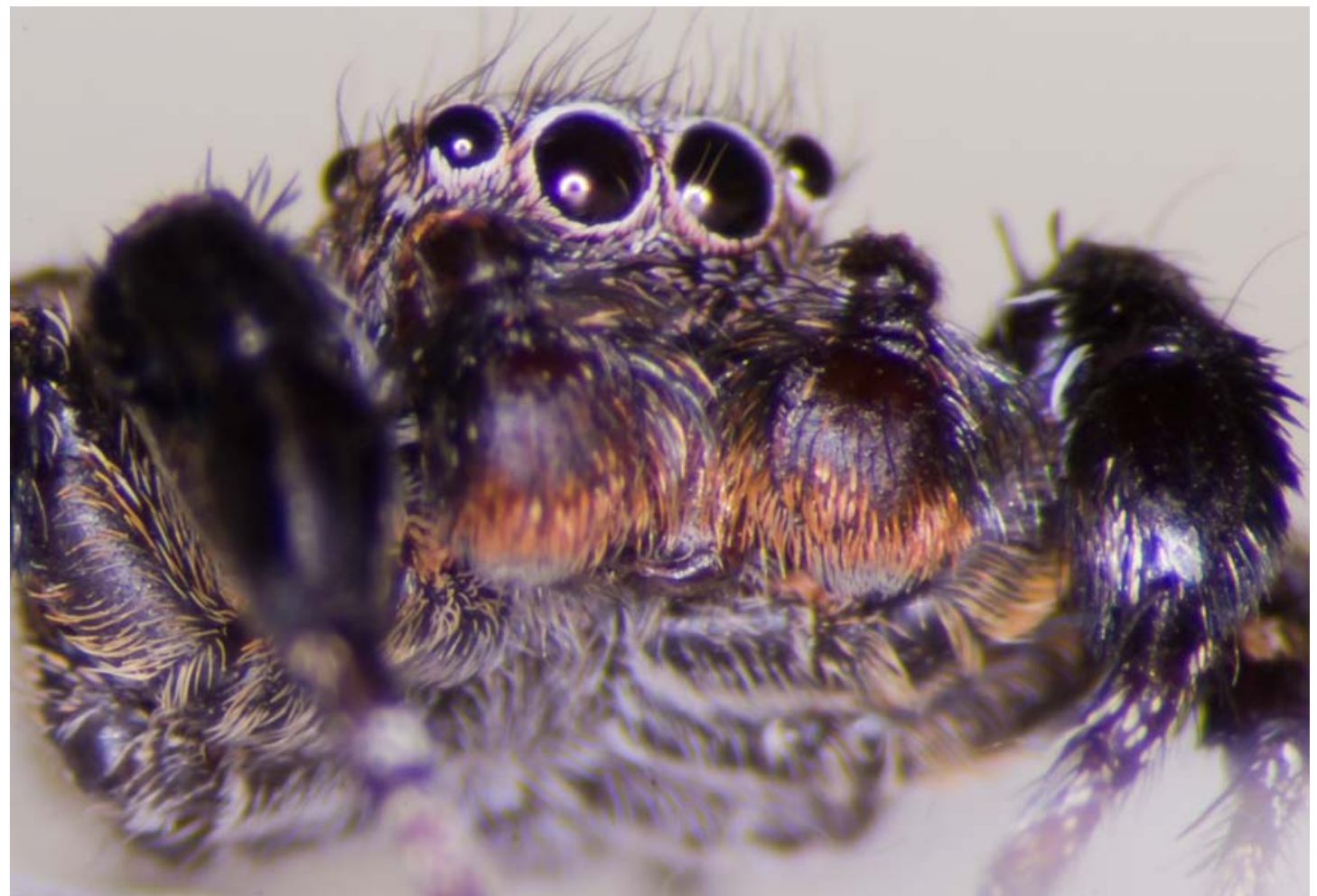

Figure 2.14. Male $H$. oregonensis Siskiyou population view of palps (beige morph). Specimen ID\# JB088. 


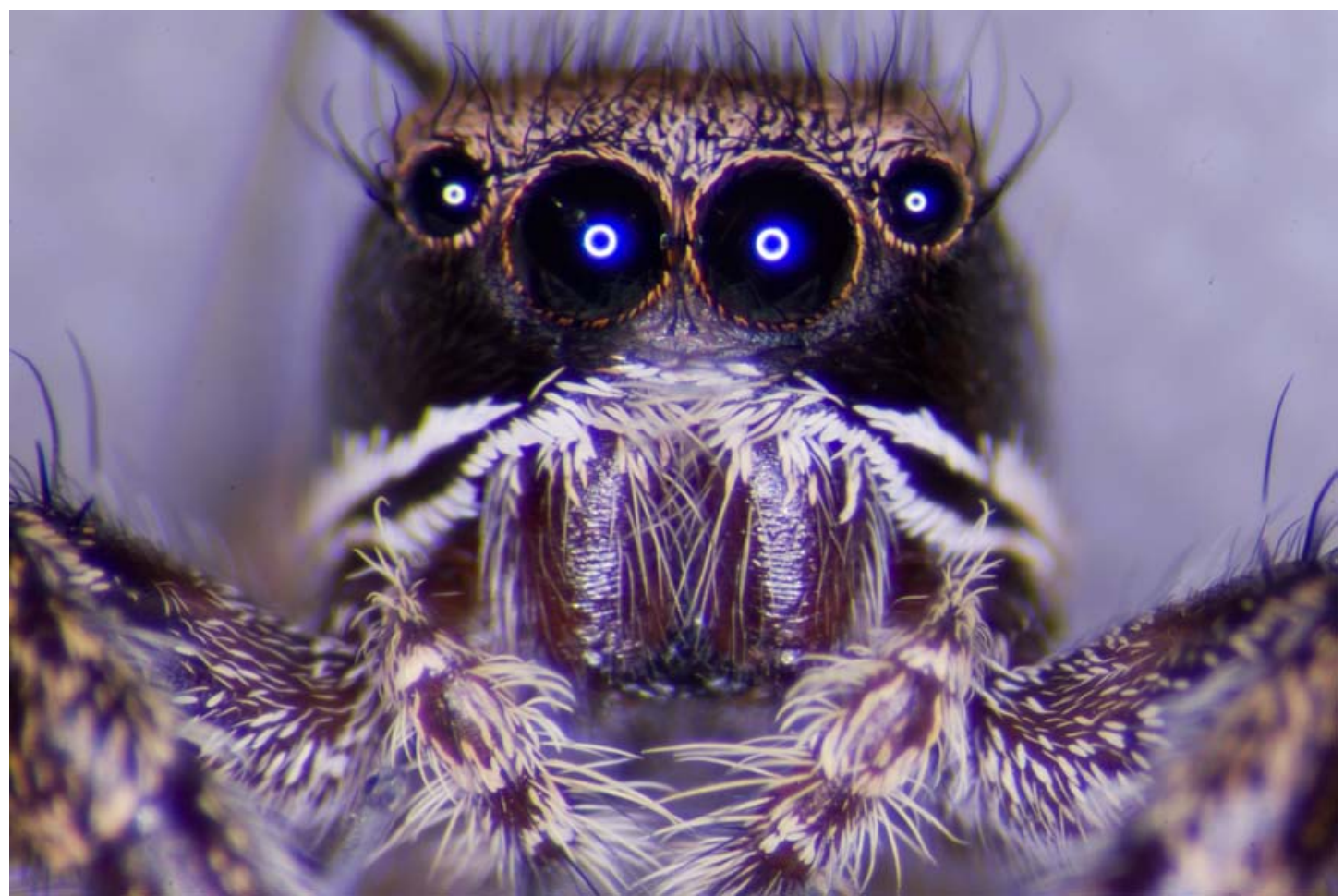

Figure 2.15. Female $H$. oregonensis Gorge population anterior aspect (beige morph). Specimen ID\# JB005. 


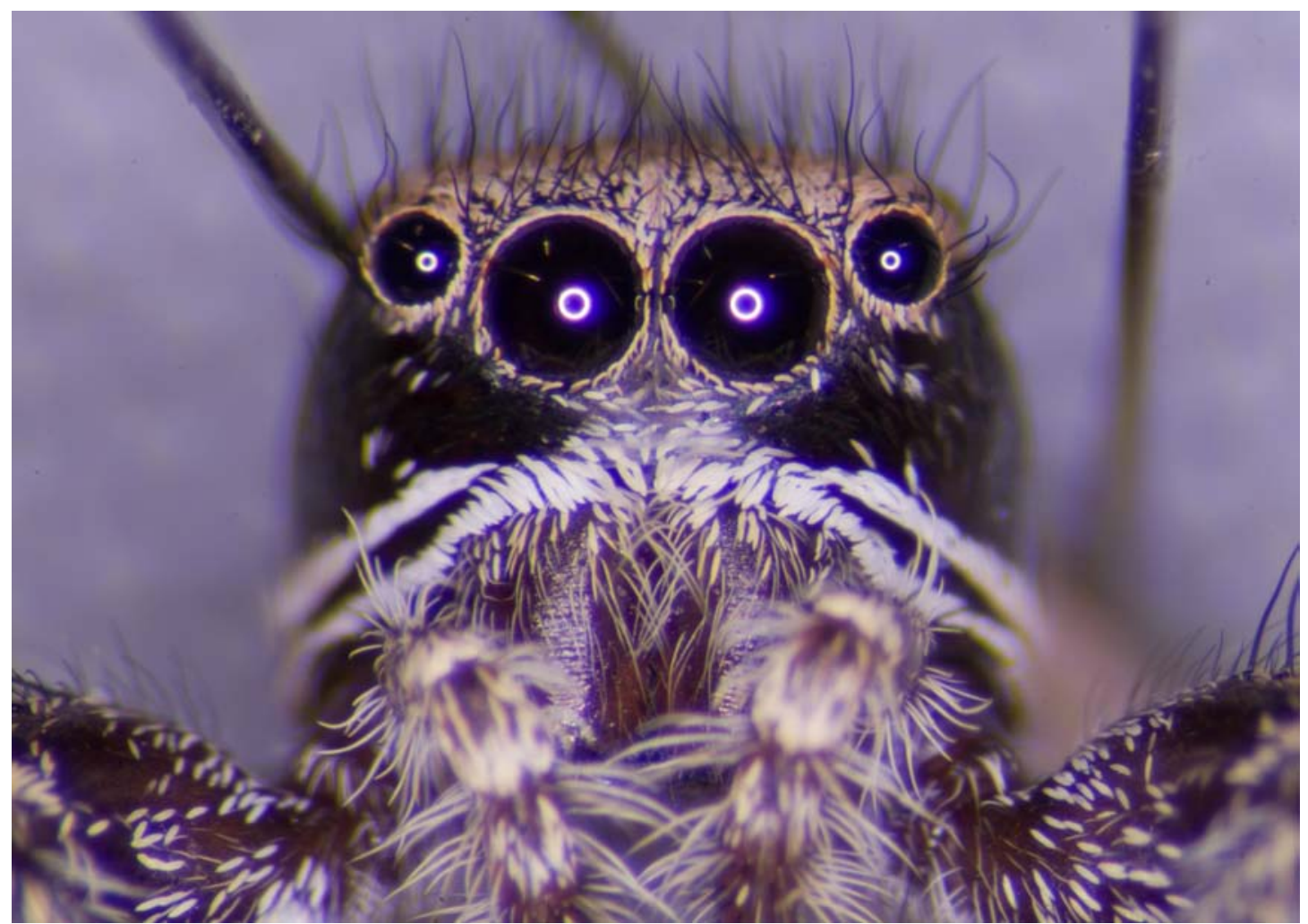

Figure 2.16. Female $H$. oregonensis Siskyiou population anterior aspect (beige morph). Specimen ID\# JB080. 


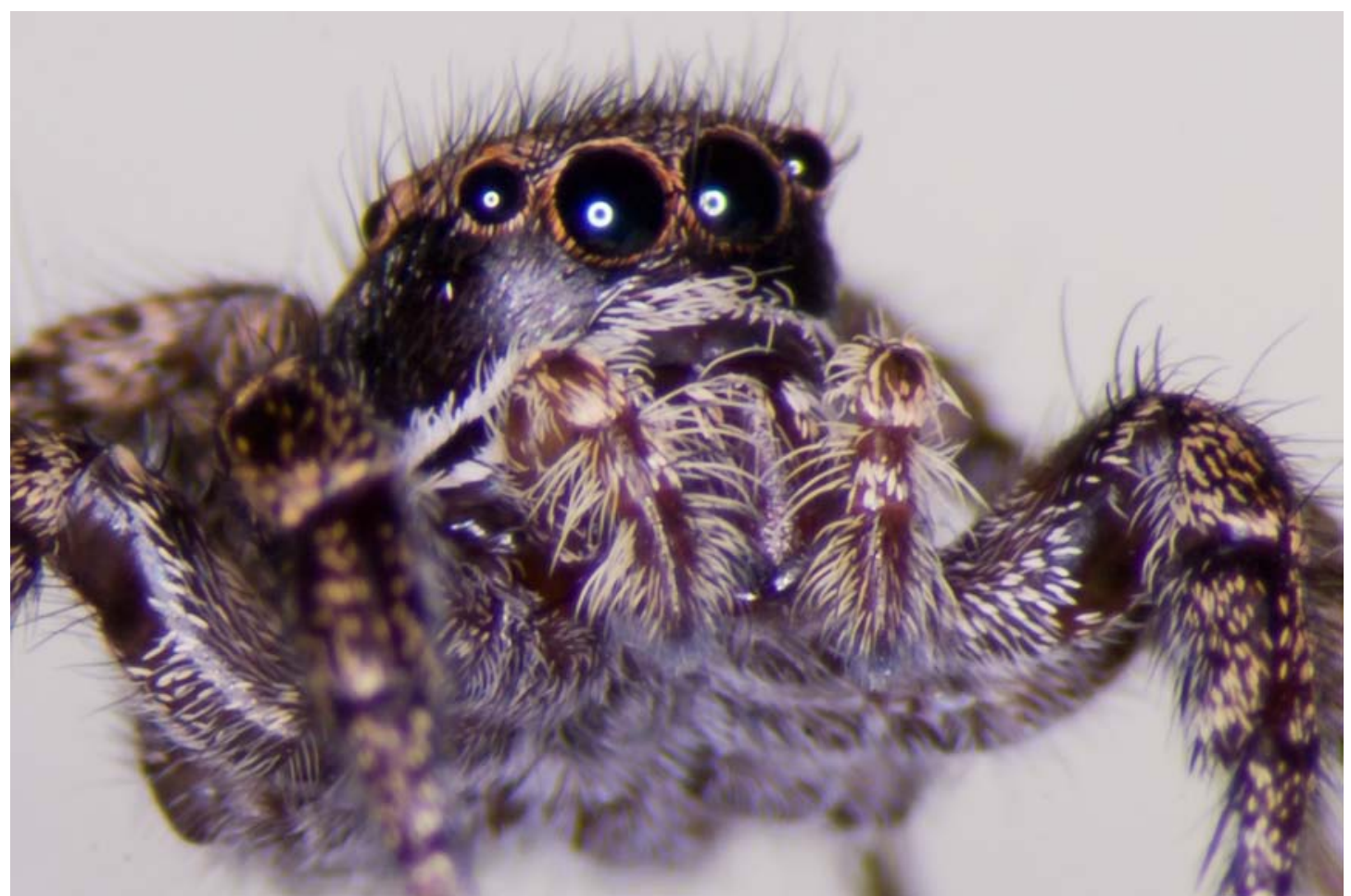

Figure 2.17. Female $H$. oregonensis Gorge population view of palps (beige morph). Specimen ID\# JB005. 


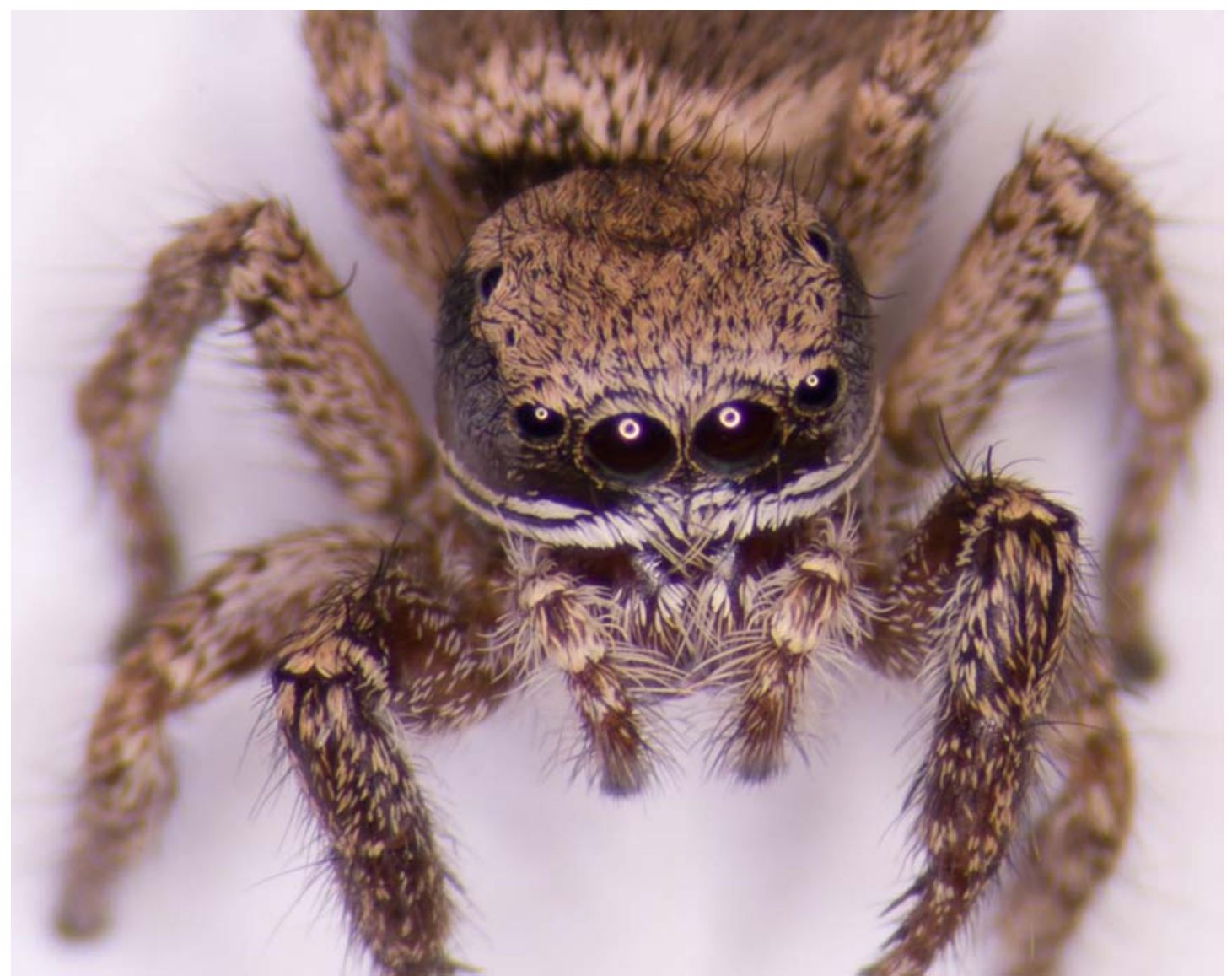

Figure 2.18. Female $H$. oregonensis Siskiyou population view of palps (beige morph). Specimen ID\# JB080. 


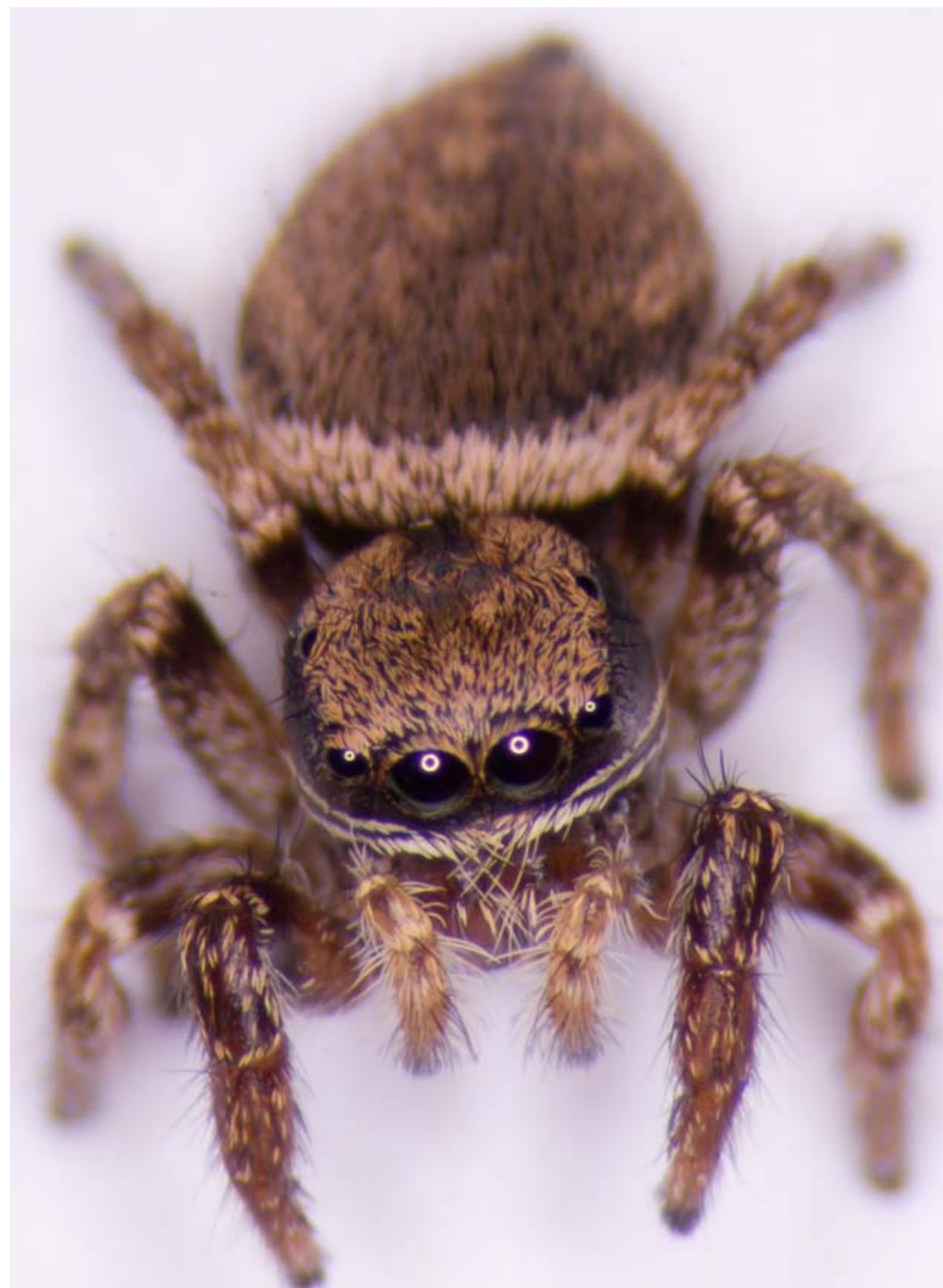

Figure 2.19. Juvenile H. oregonensis Gorge population (beige morph). Specimen ID\# JB014. 


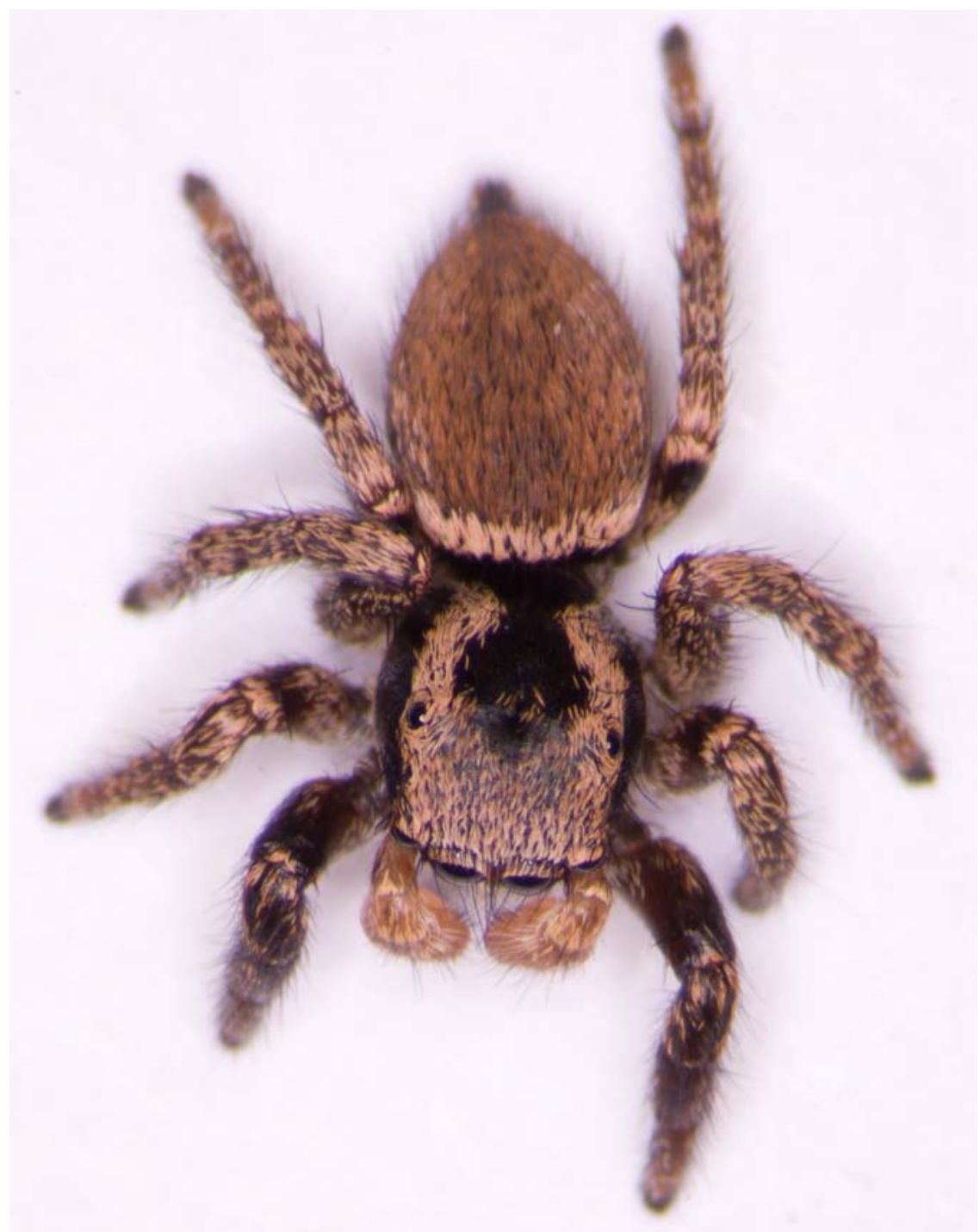

Figure 2.20. Juvenile H. oregonensis Siskiyou population (beige morph). Specimen ID\# JB092.

Bronze morph males tend to have less variation in coloration than the females. Males tend to have black and dark coppery-brown setae covering their entire body with a small patch of light-colored setae on the carapace above the anterior eyes; setae are glossy (more glossy than that of beige morph males) (Figures 2.21 and 2.22). Females have much more variable colorations and have some glossy 
black setae on the carapace, though they lack glossy setae on the abdomen (Figures 2.23 and 2.24). Bronze morph female carapaces tend to be dominated by black setae with patches of light while abdomens are generally light around the margin, dark dorsally, with a tan to orangish chevron pattern on the posterior half of the dorsal surface. This is a similar pattern to that of males, though due to the more uniform coloration of the male dorsum (black and dark brown) the pattern is more apparent among females. Anteriorly, bronze morph males have a high-contrast speckling of black and white setae on their face, similar to that of beige morph males (Figures 2.25 and 2.26). This speckling continues onto the chelicerae, however, and the dense orangish setae characteristic of beige morph males is absent (though in some individuals the white speckling is faintly orange). Like the beige morph males, bronze morph males also have white and black lines along the margin of the carapace. Bronze morph male palps are black with white speckles with little to no trace of orange setae (Figures 2.27 and 2.28). Bronze morph female anteriors and palps have no major differences from those of the beige morph females (Figures 2.29 though 2.32). A detailed analysis in color differences between the two morphs can be found in chapter 3. No juveniles were collected from the Mt. Hood population, and thus all color descriptions for bronze morph juveniles are based on the Tillamook population. As with beige morph females, bronze morph juveniles are very similar to females with respect to color; palp setae of juveniles tend to be just a little shorter and have a little less light-colored setae than those of adult females (Figure 2.33). 


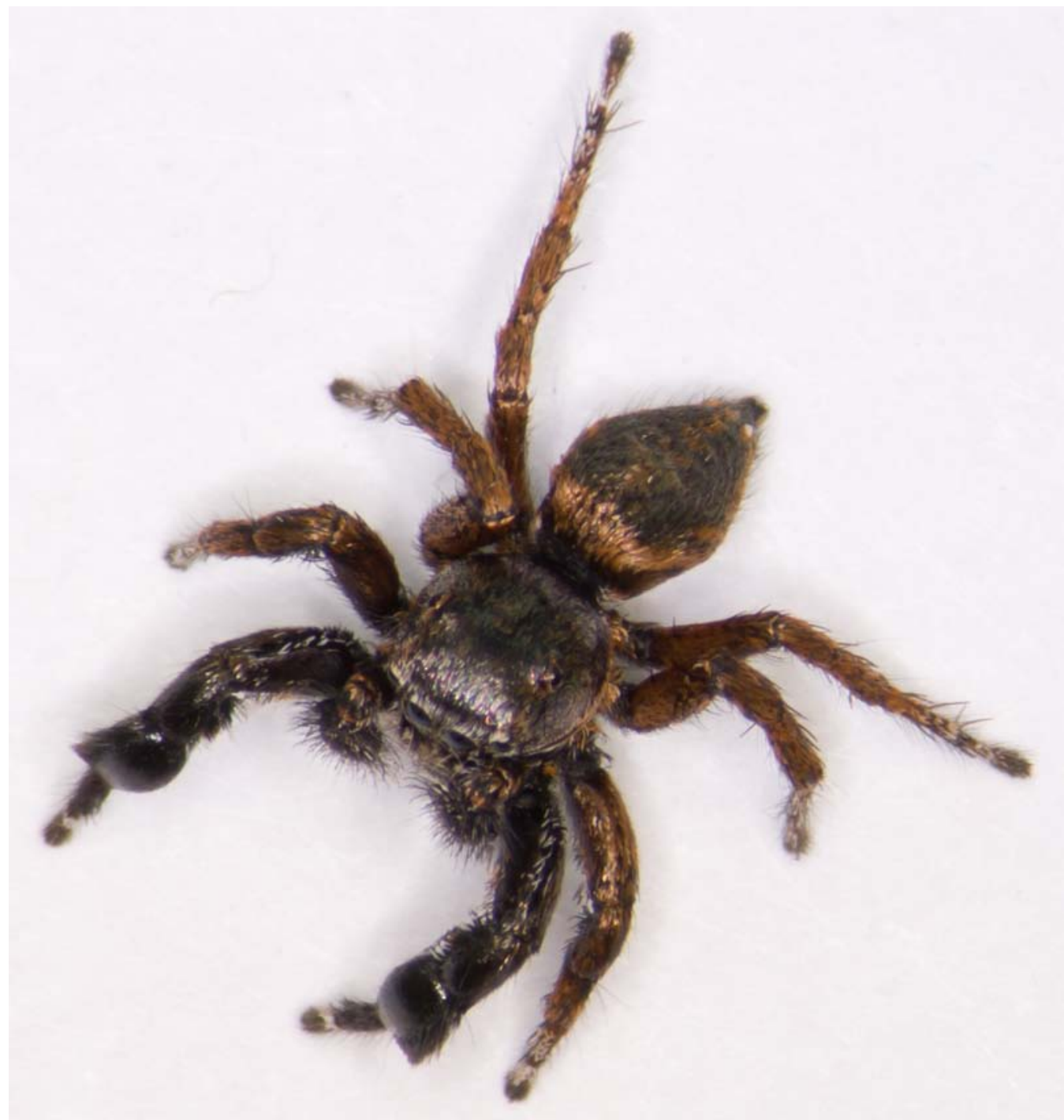

Figure 2.21. Male H. oregonensis Mt. Hood population dorsal aspect (bronze morph). Specimen ID\# JB045. 


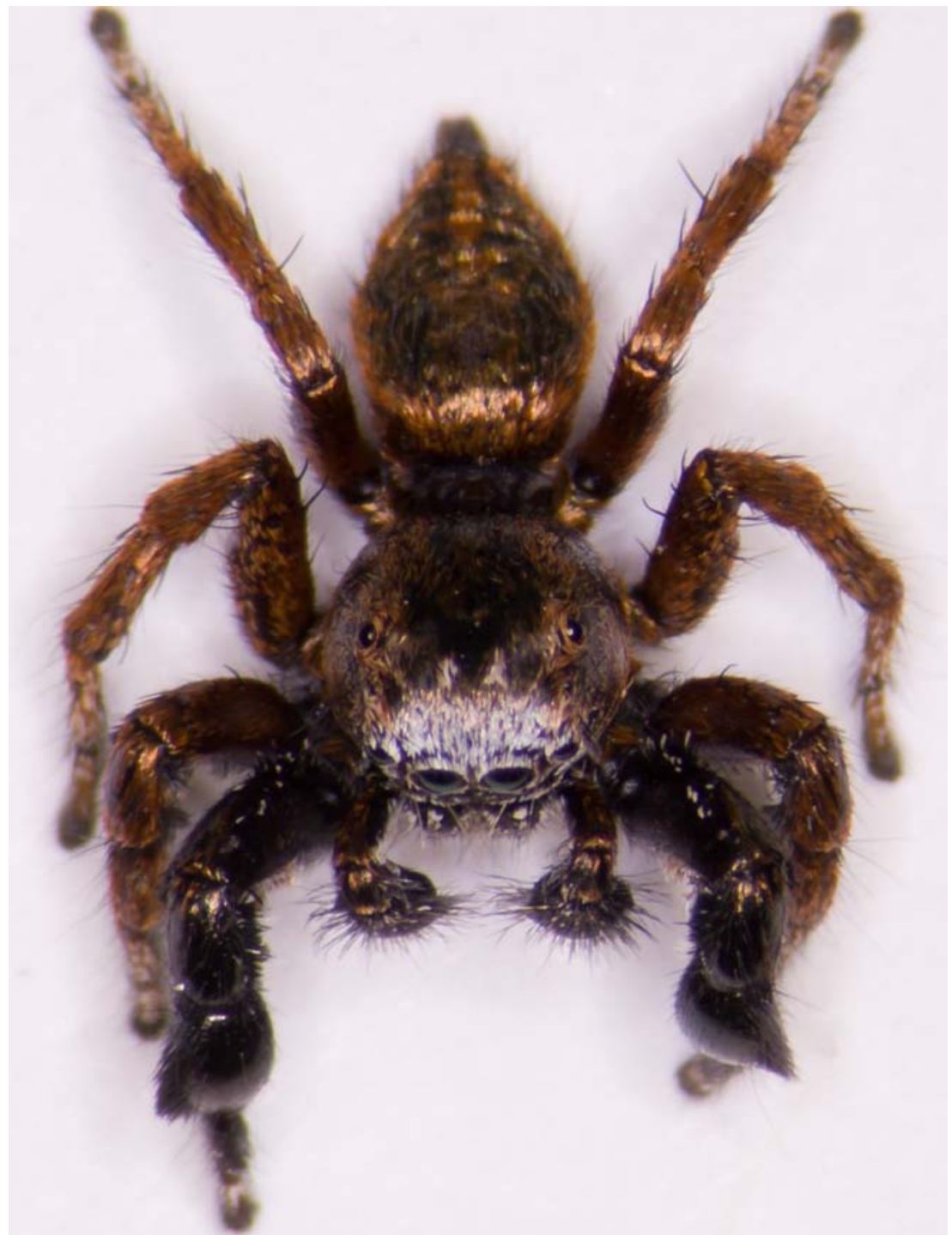

Figure 2.22. Male H. oregonensis Tillamook population dorsal aspect (bronze morph). Specimen ID\# JB061. 


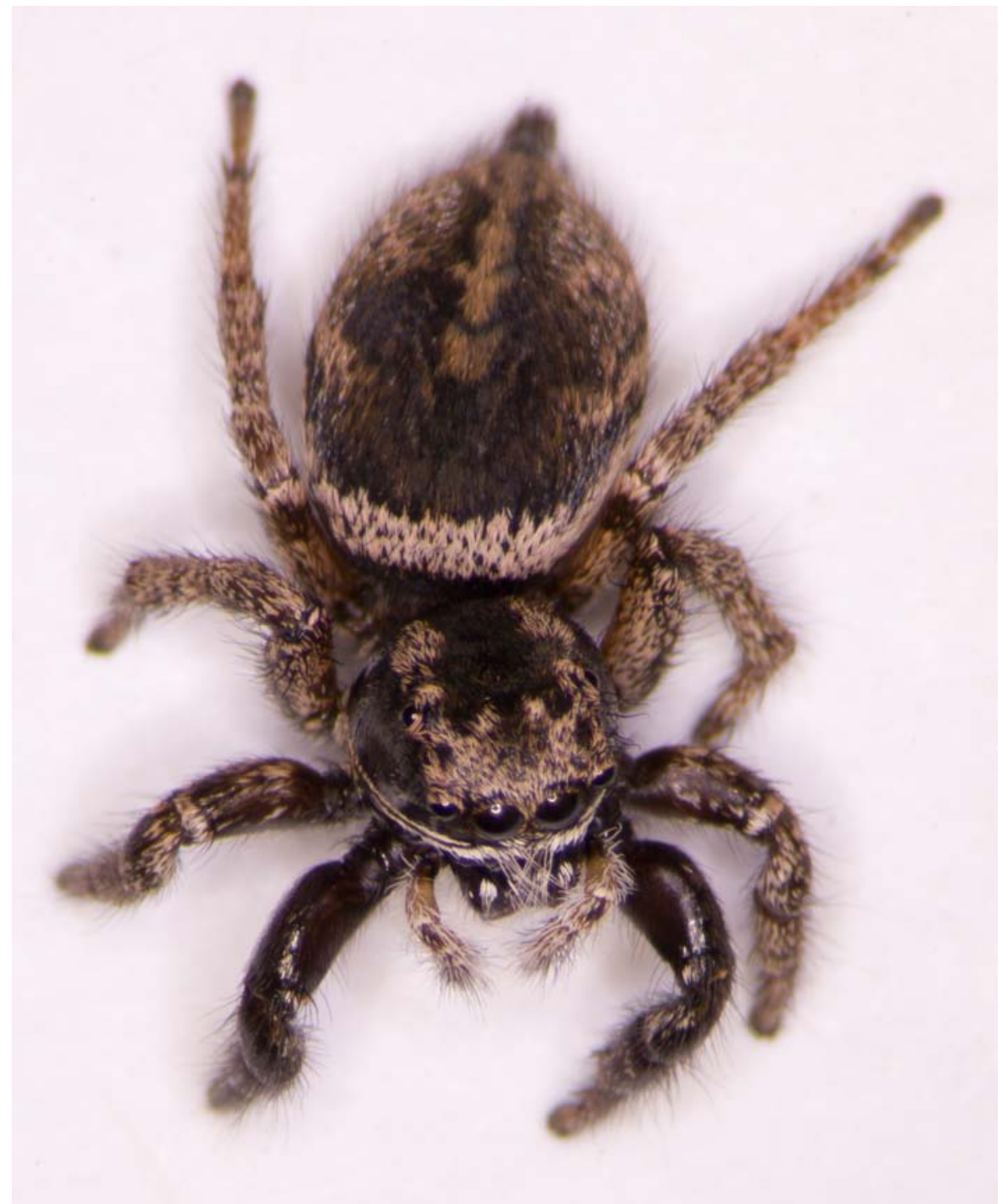

Figure 2.23. Female $H$. oregonensis Mt. Hood population dorsal aspect (bronze morph). Specimen ID\# JB041. 


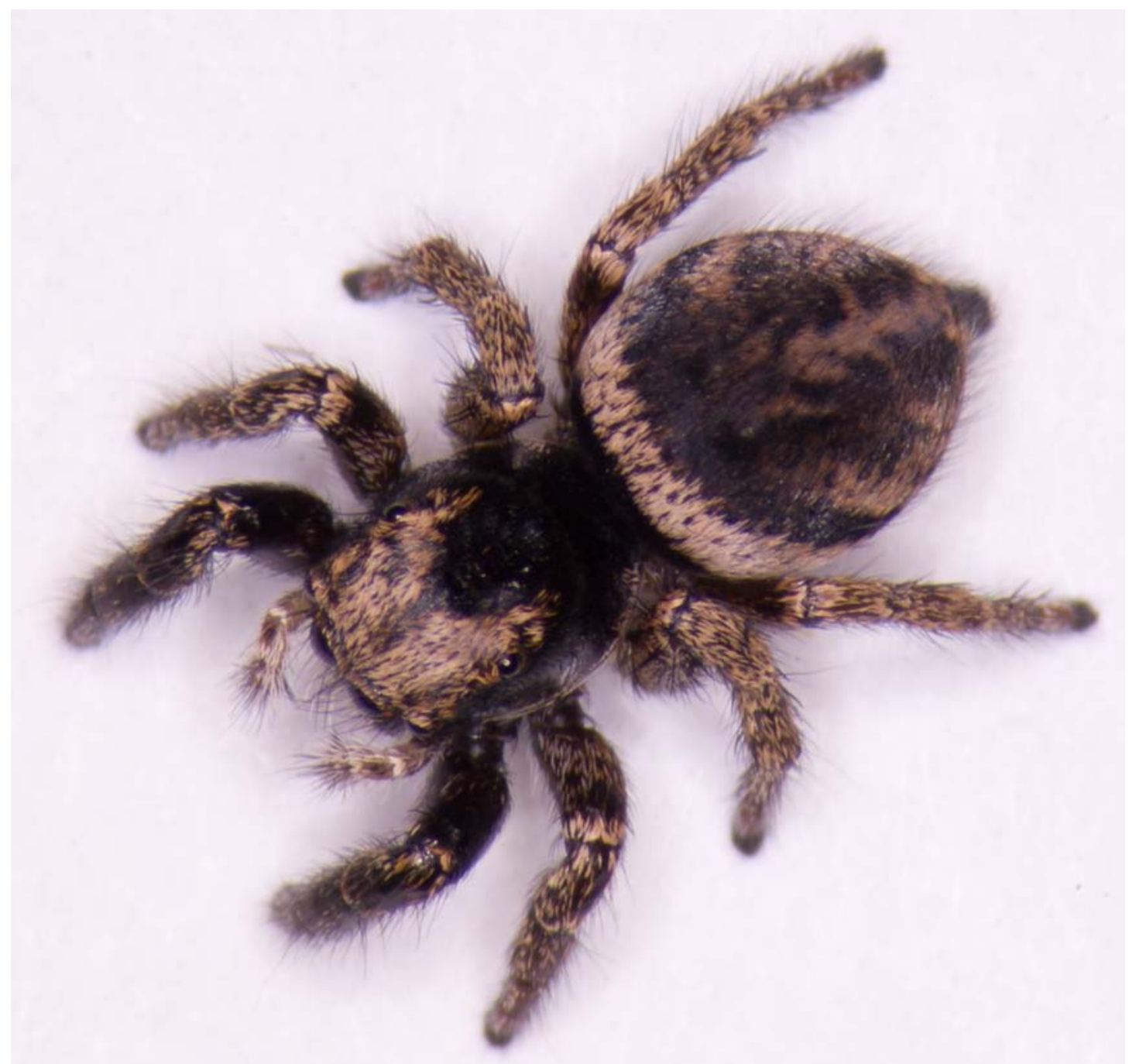

Figure 2.24. Female $H$. oregonensis Tillamook population dorsal aspect (bronze morph). Specimen ID\# JB062. 


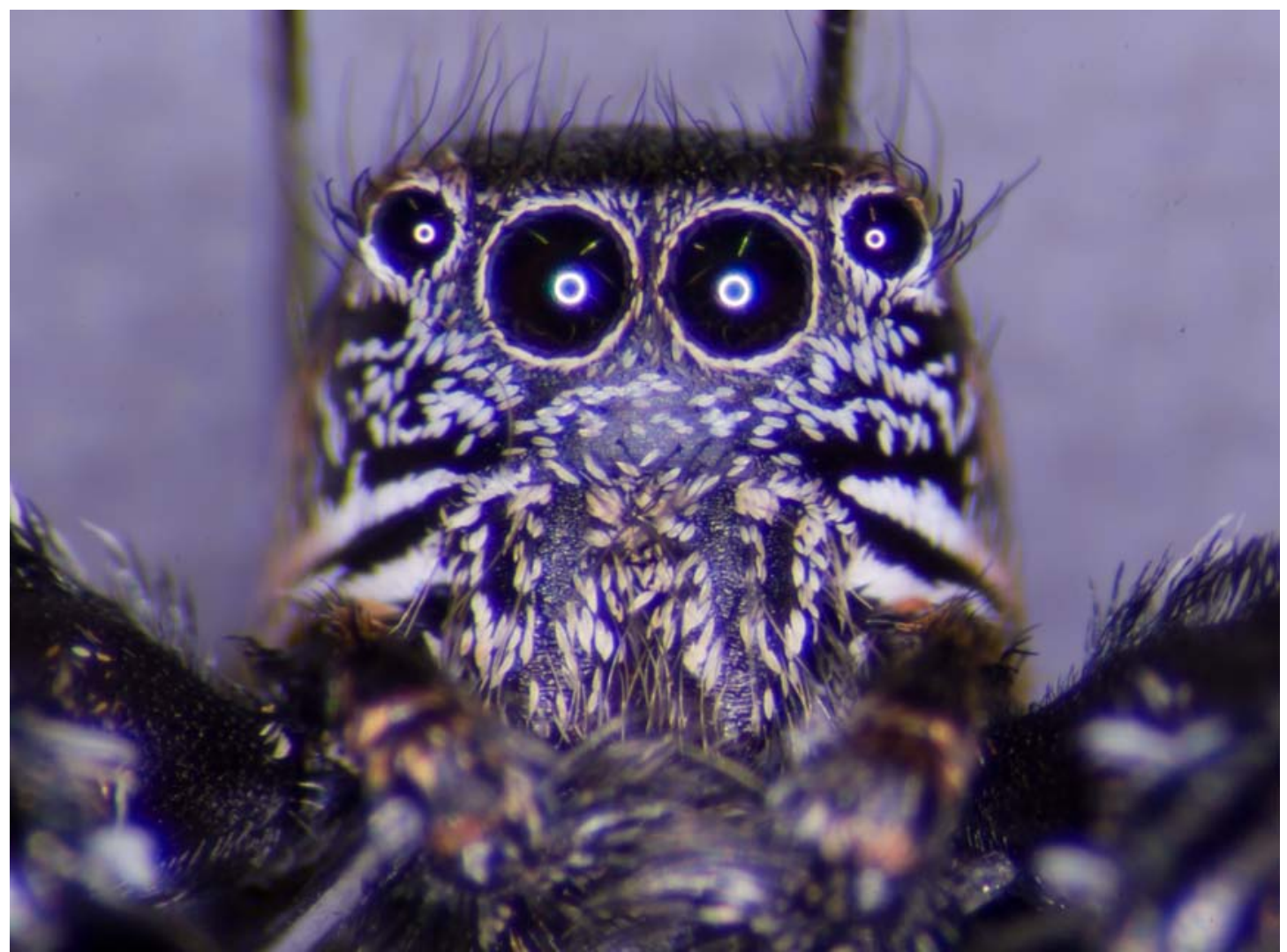

Figure 2.25. Male H. oregonensis Mt. Hood population anterior aspect (bronze morph). Specimen ID\# JB045. 


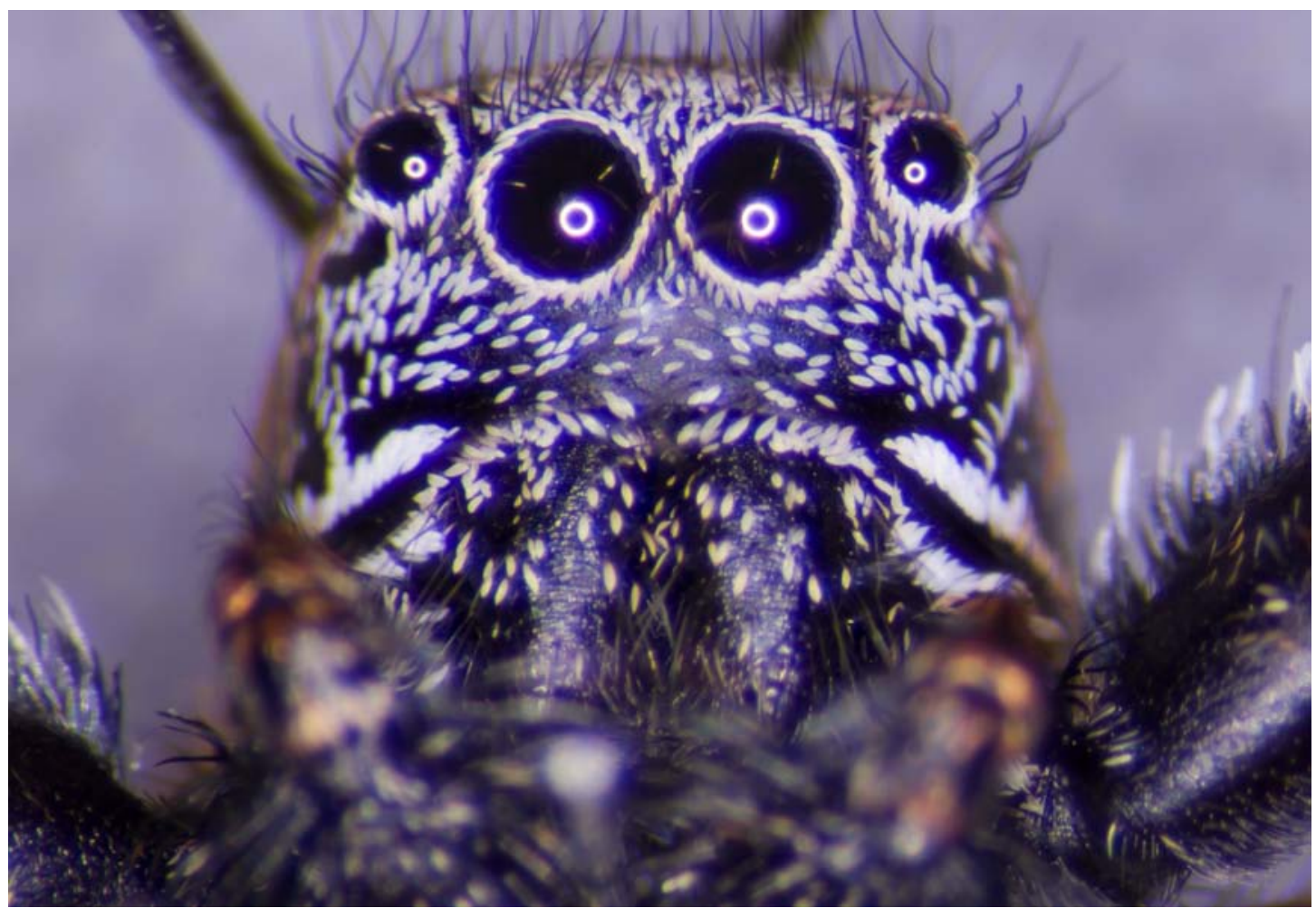

Figure 2.26. Male $H$. oregonensis Tillamook population anterior aspect (bronze morph). Specimen ID\# JB061. 


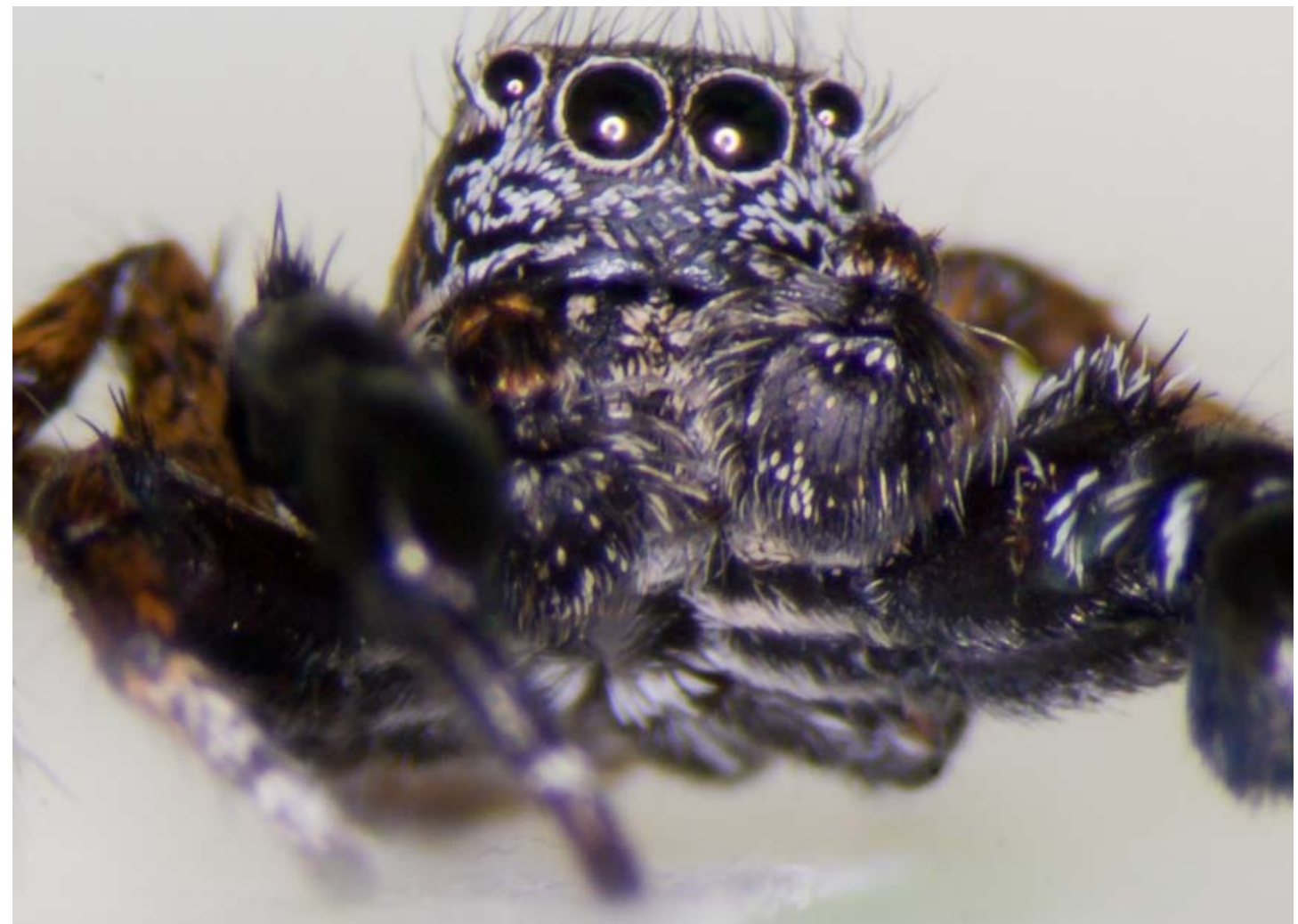

Figure 2.27. Male $H$. oregonensis Mt. Hood population view of palps (bronze morph). Specimen ID\# JB045.

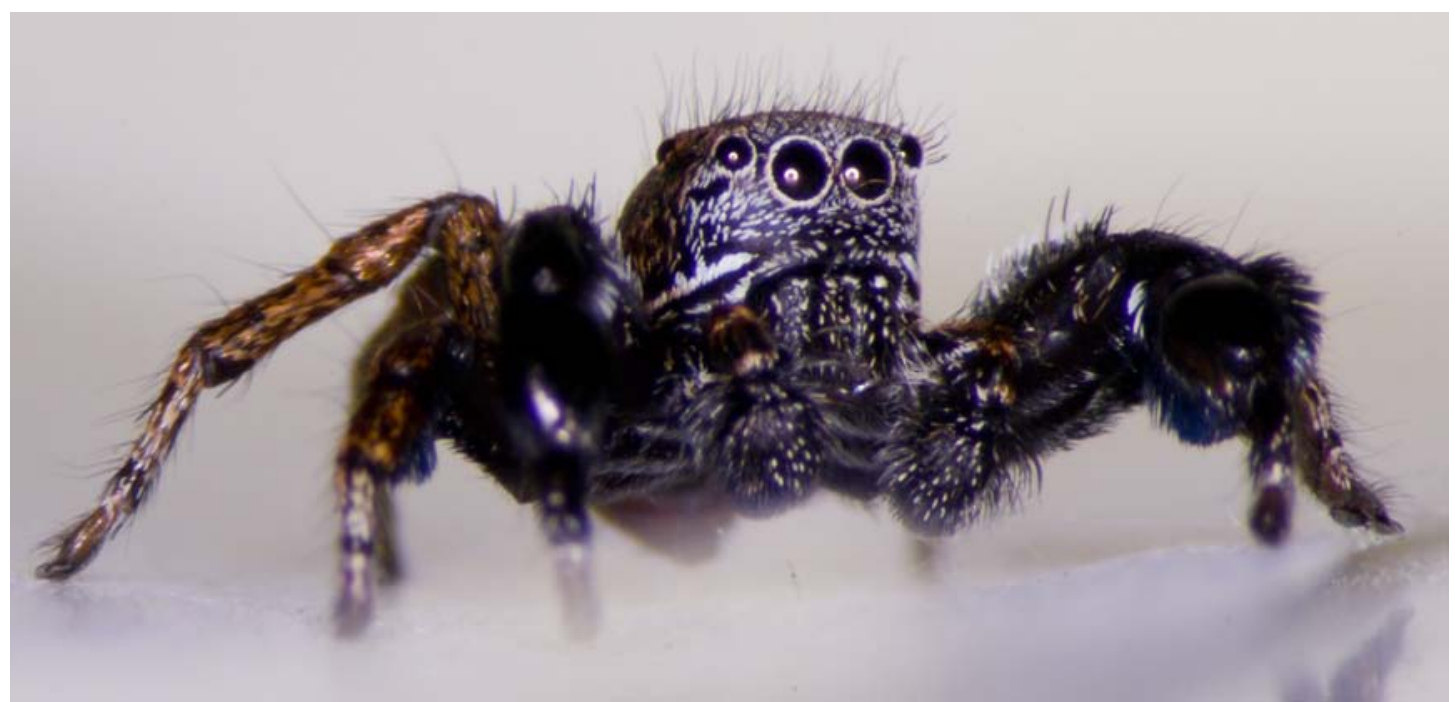

Figure 2.28. Male $H$. oregonensis Tillamook population view of palps (bronze morph). Specimen ID\# JB061. 


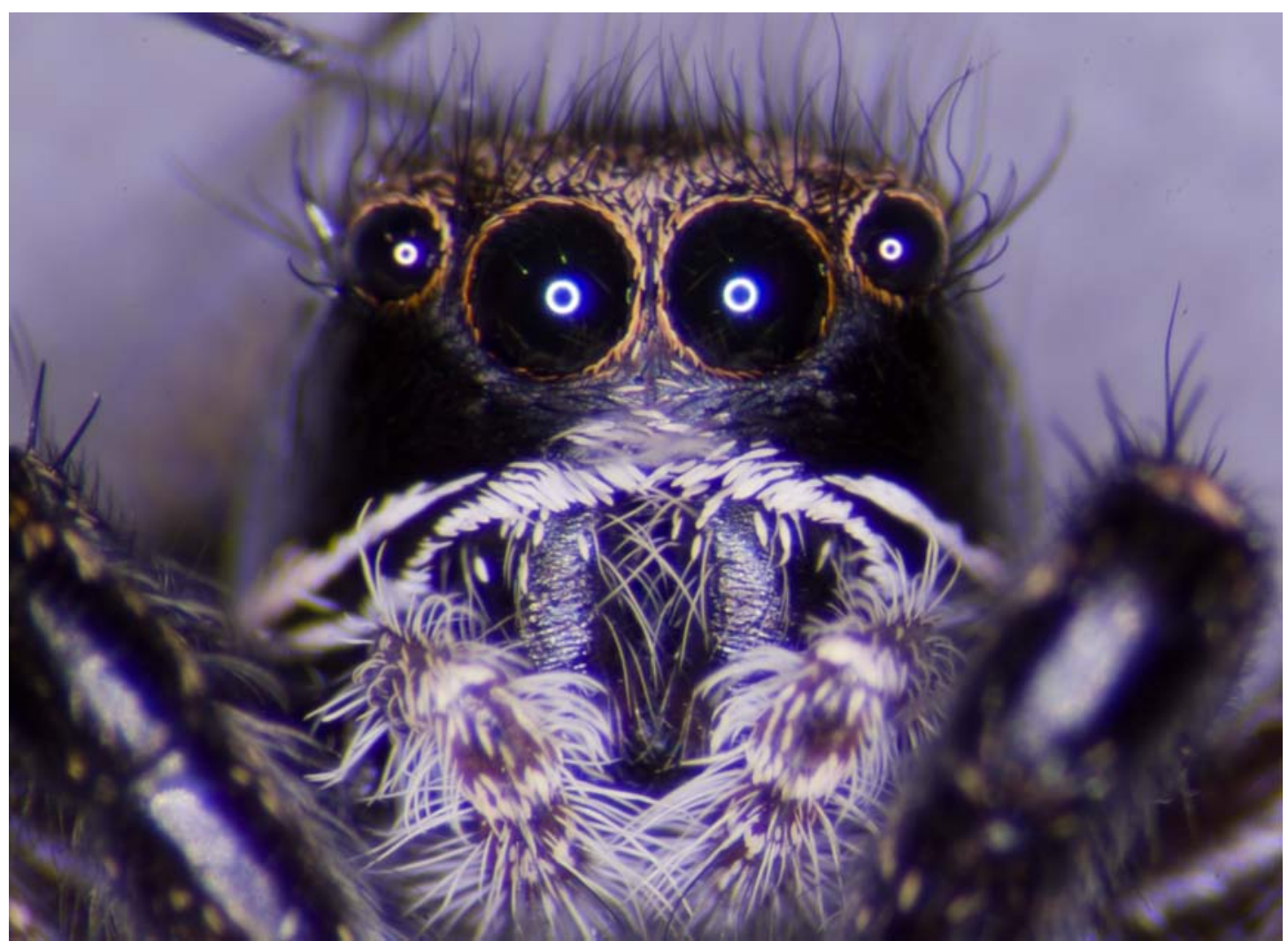

Figure 2.29. Female $H$. oregonensis Mt. Hood population anterior aspect (bronze morph). Specimen ID\# JB041. 


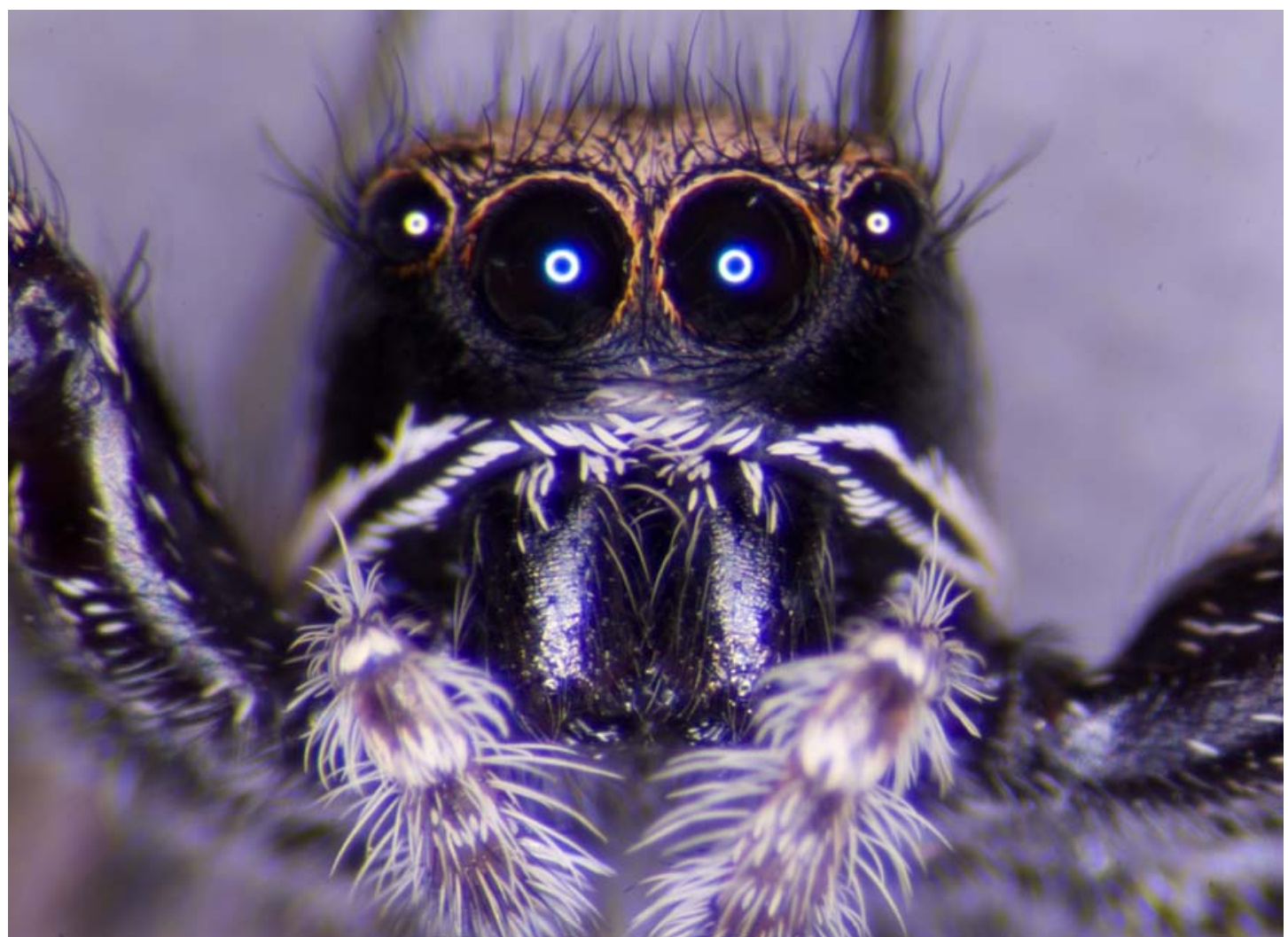

Figure 2.30. Female $H$. oregonensis Tillamook population anterior aspect (bronze morph). Specimen ID\# JB062. 


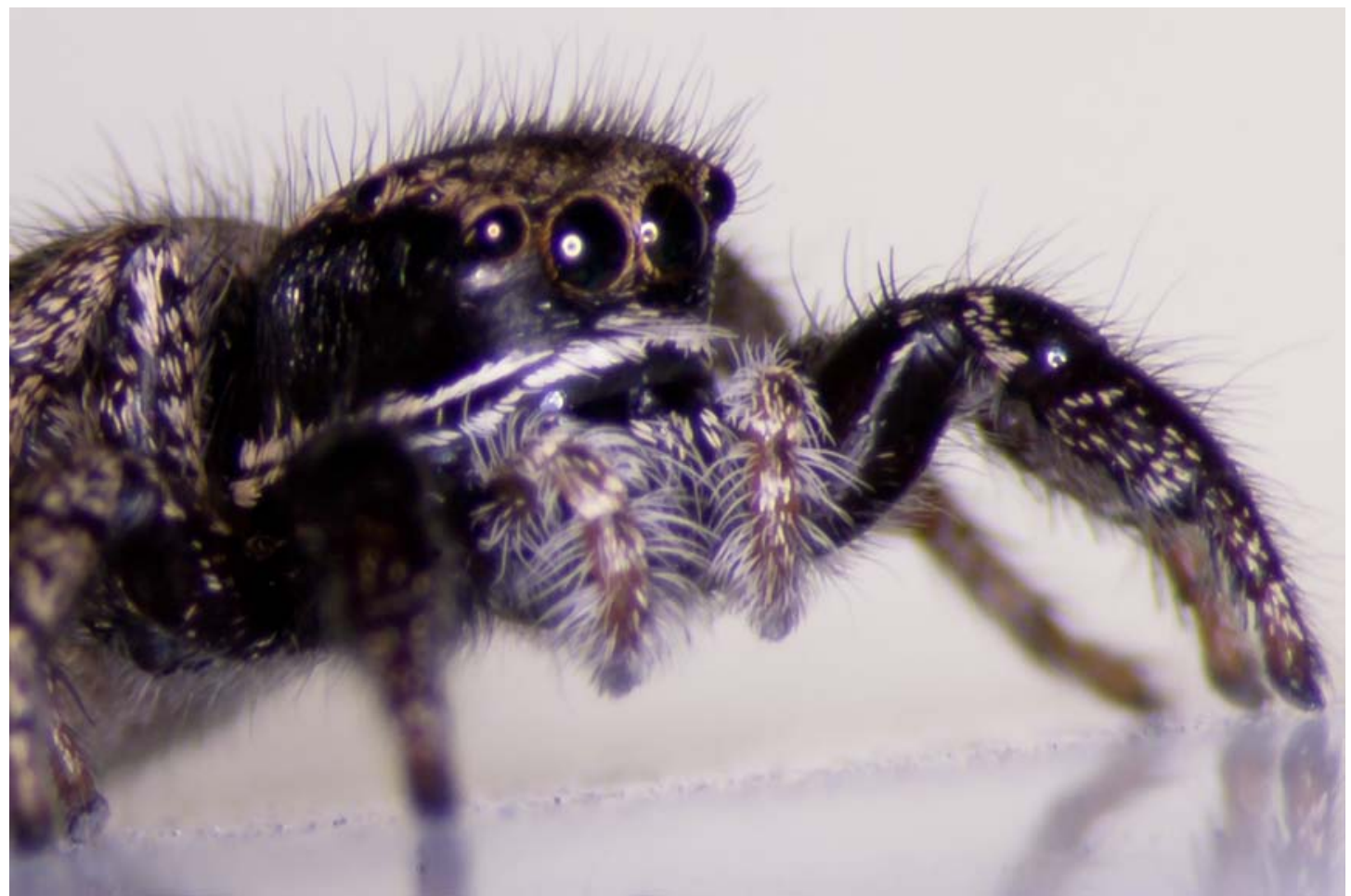

Figure 2.31. Female $H$. oregonensis Mt. Hood population view of palps (bronze morph). Specimen ID\# JB041.

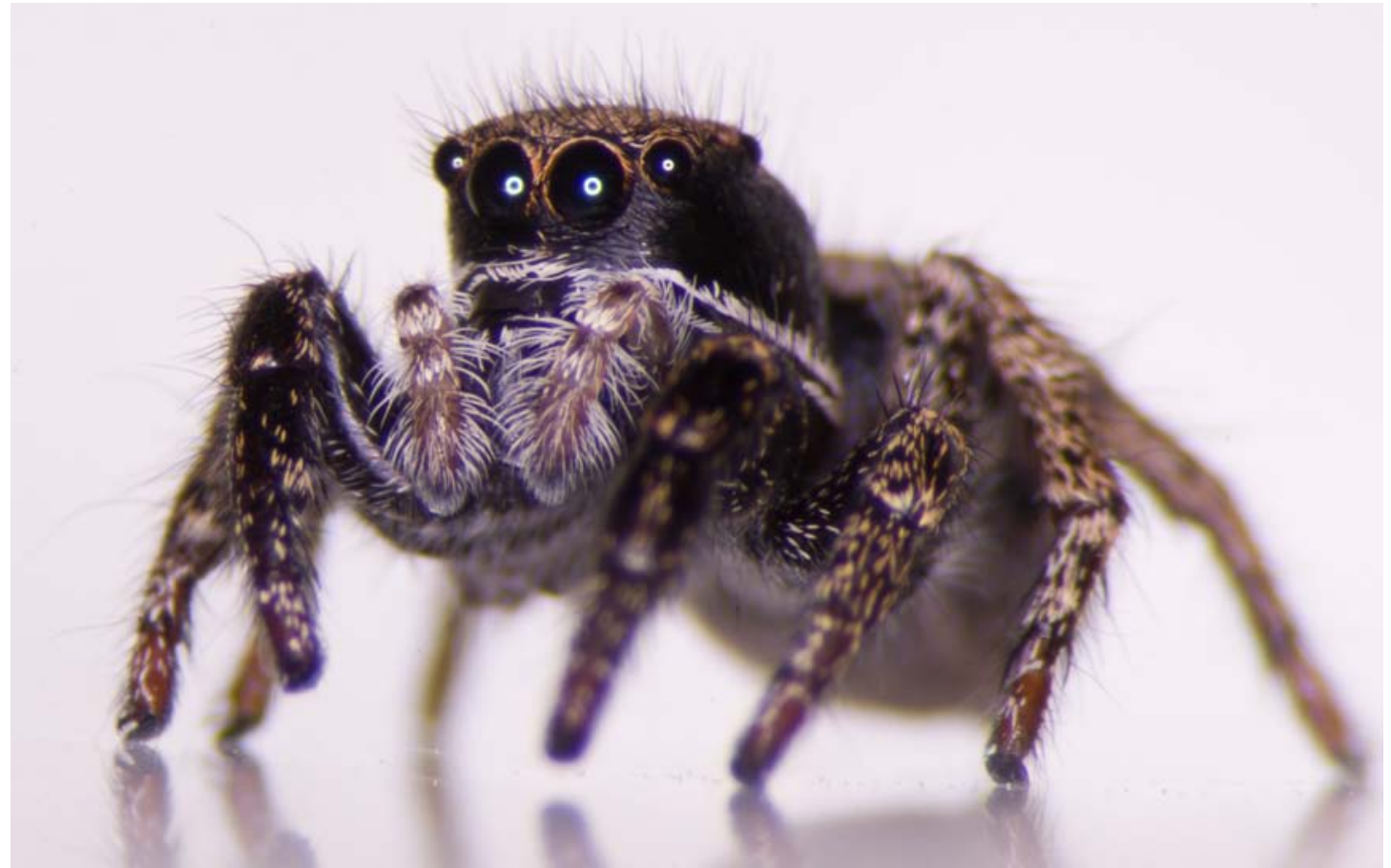

Figure 2.32. Female $H$. oregonensis Tillamook population view of palps (bronze morph). Specimen ID\# JB062. 


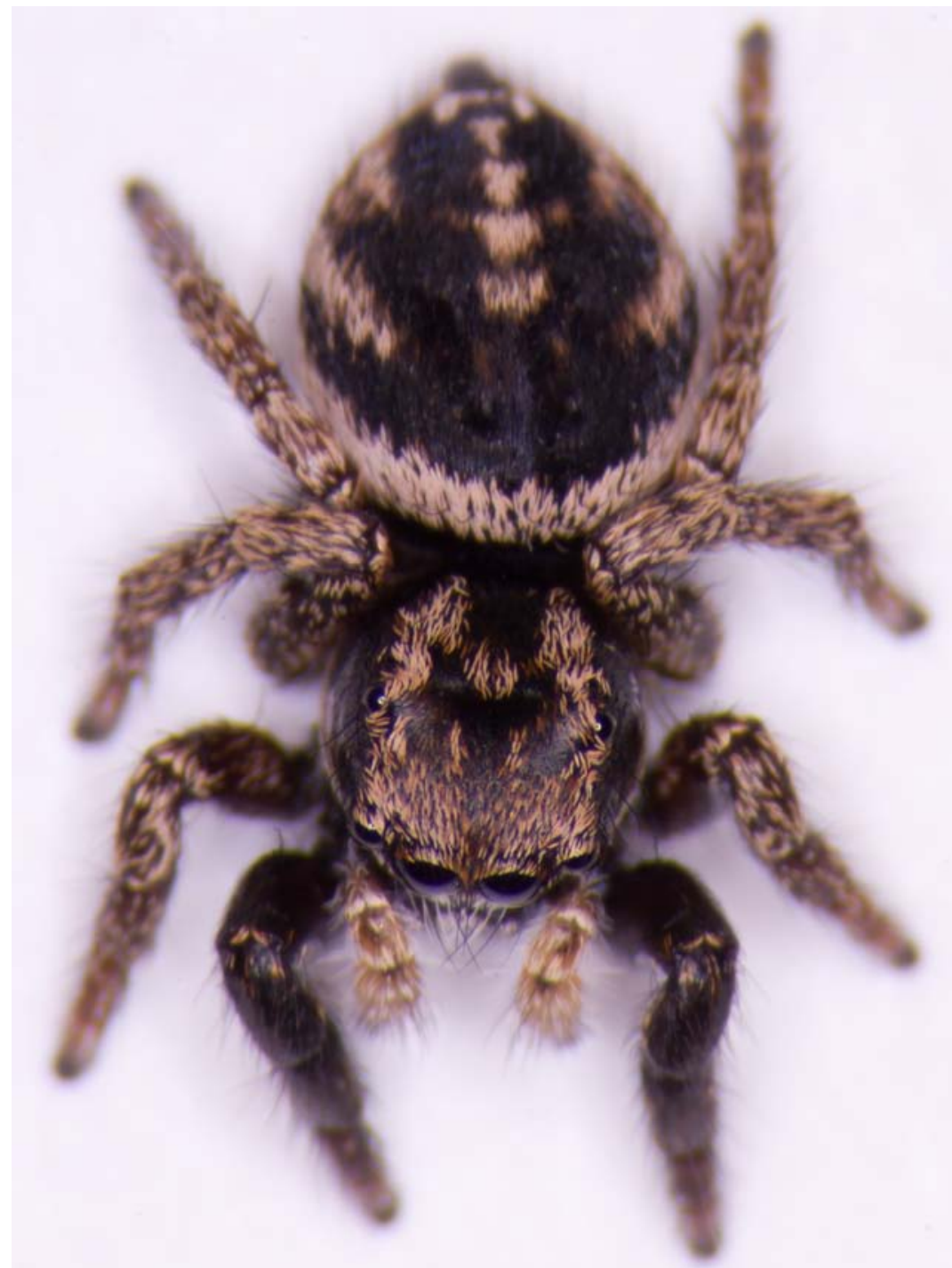

Figure 2.33. Juvenile $H$. oregonensis Tillamook population (bronze morph). Specimen ID\# JB073.

\section{Miscellaneous Observations}

Out of all individuals I encountered at the four main sites, $84 \%$ (76 out of 90) were adult. I found males far more often than females at all sites, accounting for $76 \%$ of all adults captured (58 out of 76 ). One site (the Tillamook population) had no adult females. I found $H$. oregonensis as early in the day as 0720 (about 1.75 hours 
after sunrise) and as late as 2047 (just after sunset); with the exception of the early morning, spider activity was fairly consistent throughout the daylight hours with a moderate peak in the early afternoon (Figure 2.34). I found males, females, and juveniles throughout the day; other than a slight tendency for males to be more common than females and juveniles around midday, there was no marked temporal segregation (Figure 2.35). I generally found adult female spiders to be motionless (61\% of the time); besides one instance of feeding, the remainder of the encounters were of spiders on the move. Males were largely stationary as well (57\% of the time). All of the remaining encounters of males were on the move, with a single exception: I found one male engaged in a conflict with a male $H$. hirsutus; the former chased the latter for a distance of about two meters (Figure 2.36). I often found spiders proximal to conspecifics, oftentimes between one and four meters from another individual. I found two Gorge population females (one juvenile at the time of collection) to each have a louse attached to their cephalothorax, lodged between the cephalothorax and the abdomen. While I preserved the adult soon after capture, in the case of the juvenile, the louse remained attached for seven months in the same place through three molts of the spider, at which point the spider reached adulthood and was preserved (Figure 2.37). The most common potential predators of $H$. oregonensis observed by far while collecting were hymenopterans; I often saw pompilid spider wasps roaming over the rocks, though generally not at times when $H$. oregonensis were active. I spotted a small number of northern alligator lizards 
(Elgaria coerulia) at the Tillamook collection site; also I infrequently encountered western fence lizards (Sceloporus occidentalis) at the Siskiyou site. There was no significant difference among males, females, and juveniles in their preference for sun exposure (characterized by number of encounters in habitat that was exposed, shaded, or at the edge of exposed and shaded); chi-square $p$-value $=0.761$. Out of all the females that I collected, only two females - one from the Gorge population and one from the Mt. Hood population - were noticeably gravid (as evinced by their greatly distended abdomens) upon collection; both laid eggs in captivity (before being measuring with the spectrometer) and aggressively guarded their eggs.

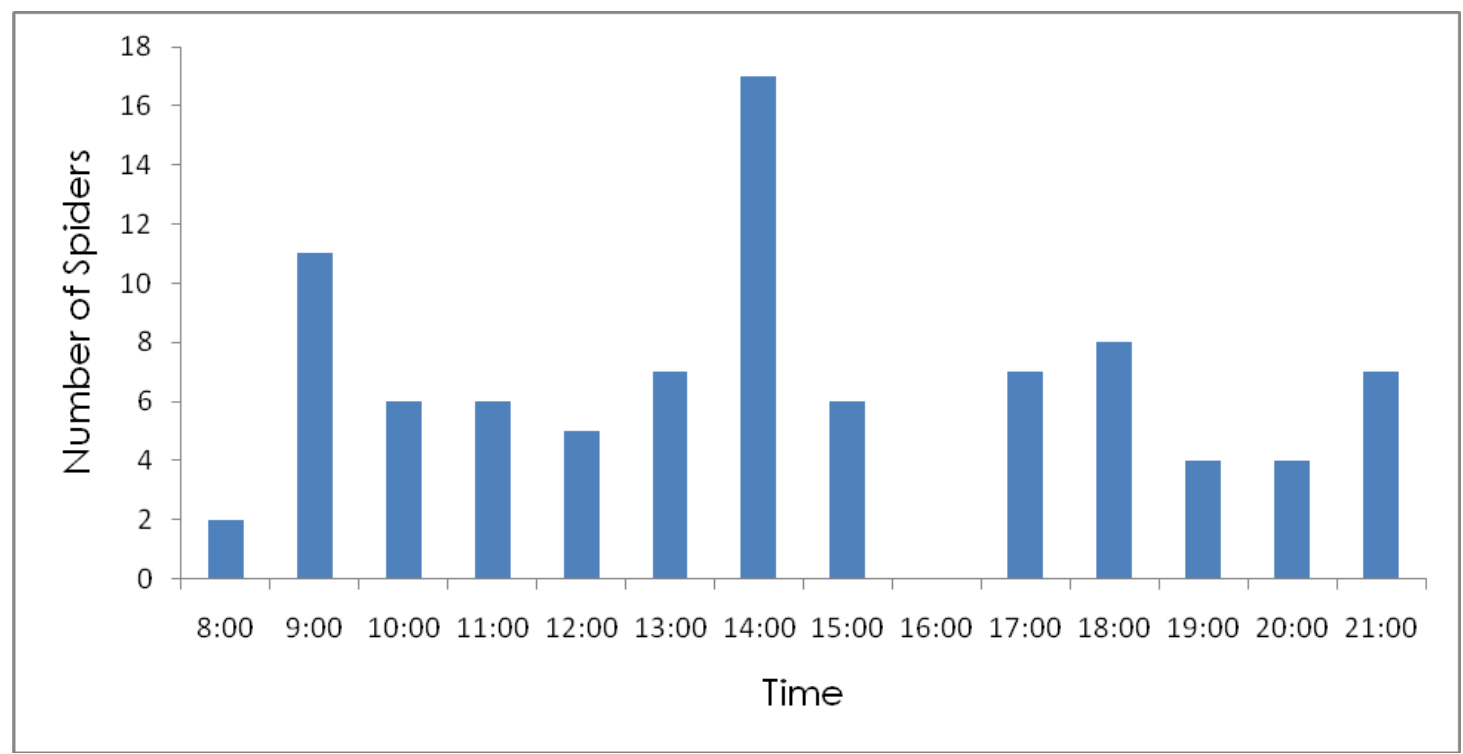

Figure 2.34. Number of $H$. oregonensis encountered by time of day. 


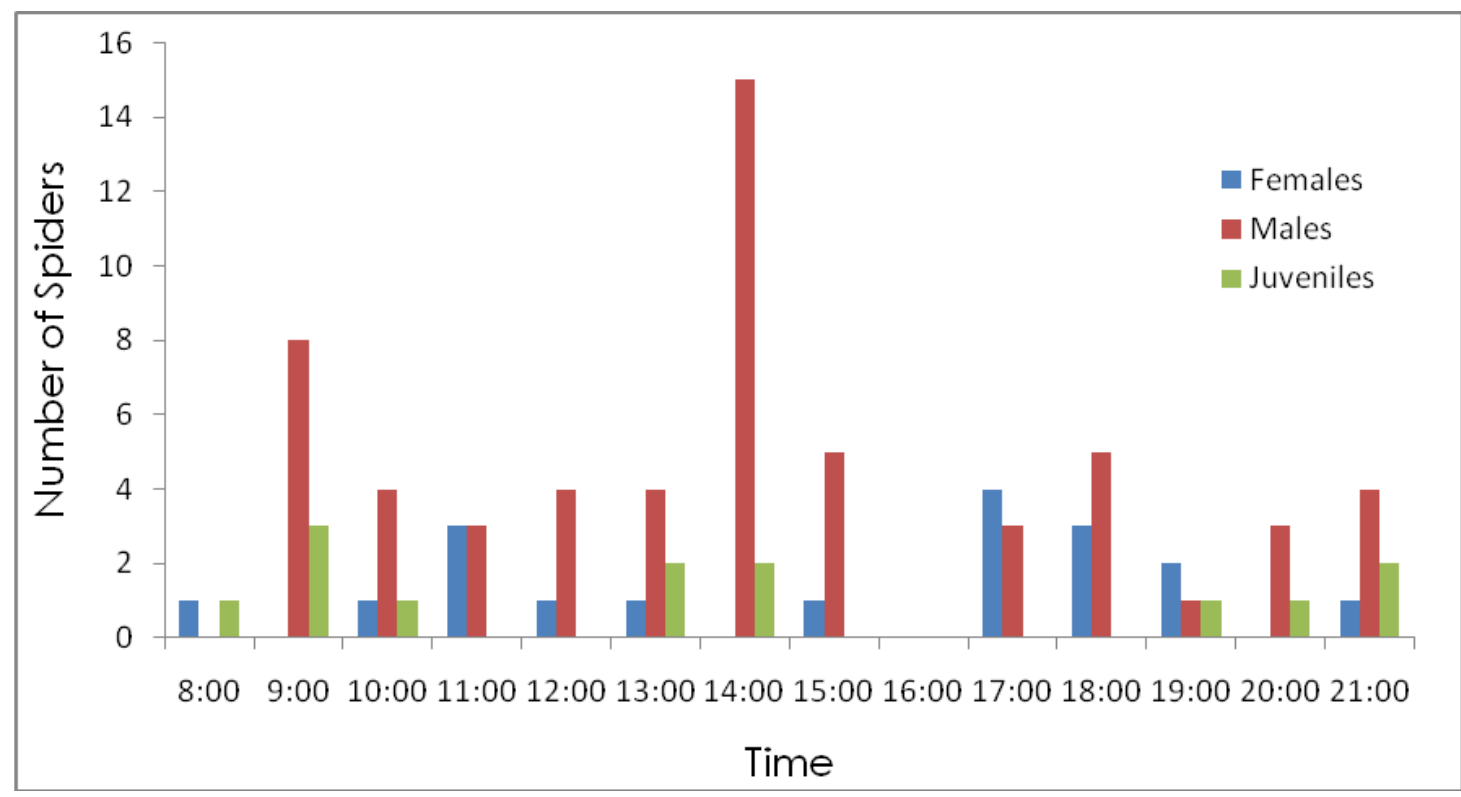

Figure 2.35. Number of $H$. oregonensis encountered by time of day, grouped by sex and developmental stage.

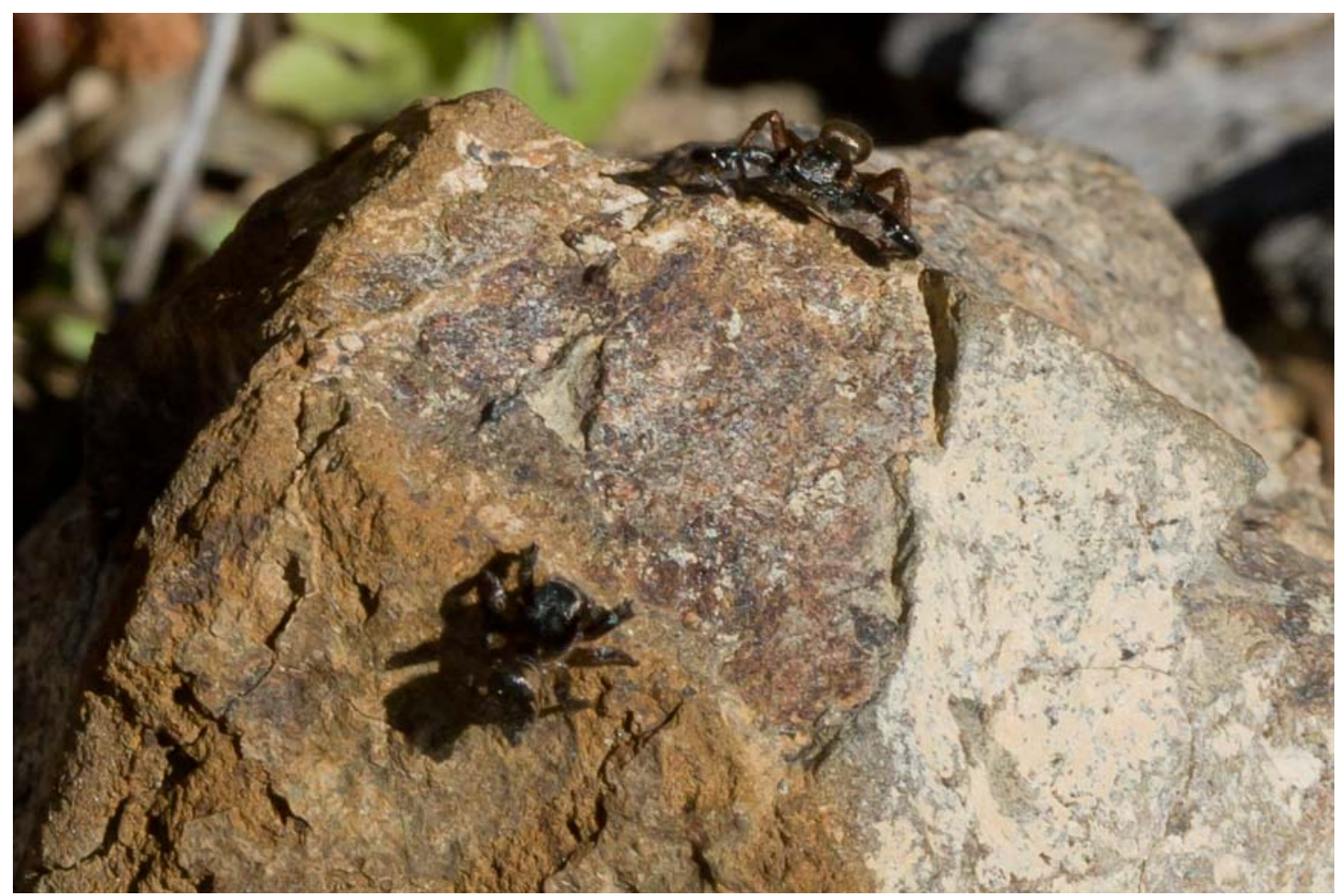

Figure 2.36. $H$. oregonensis and $H$. hirsutus conflict. 


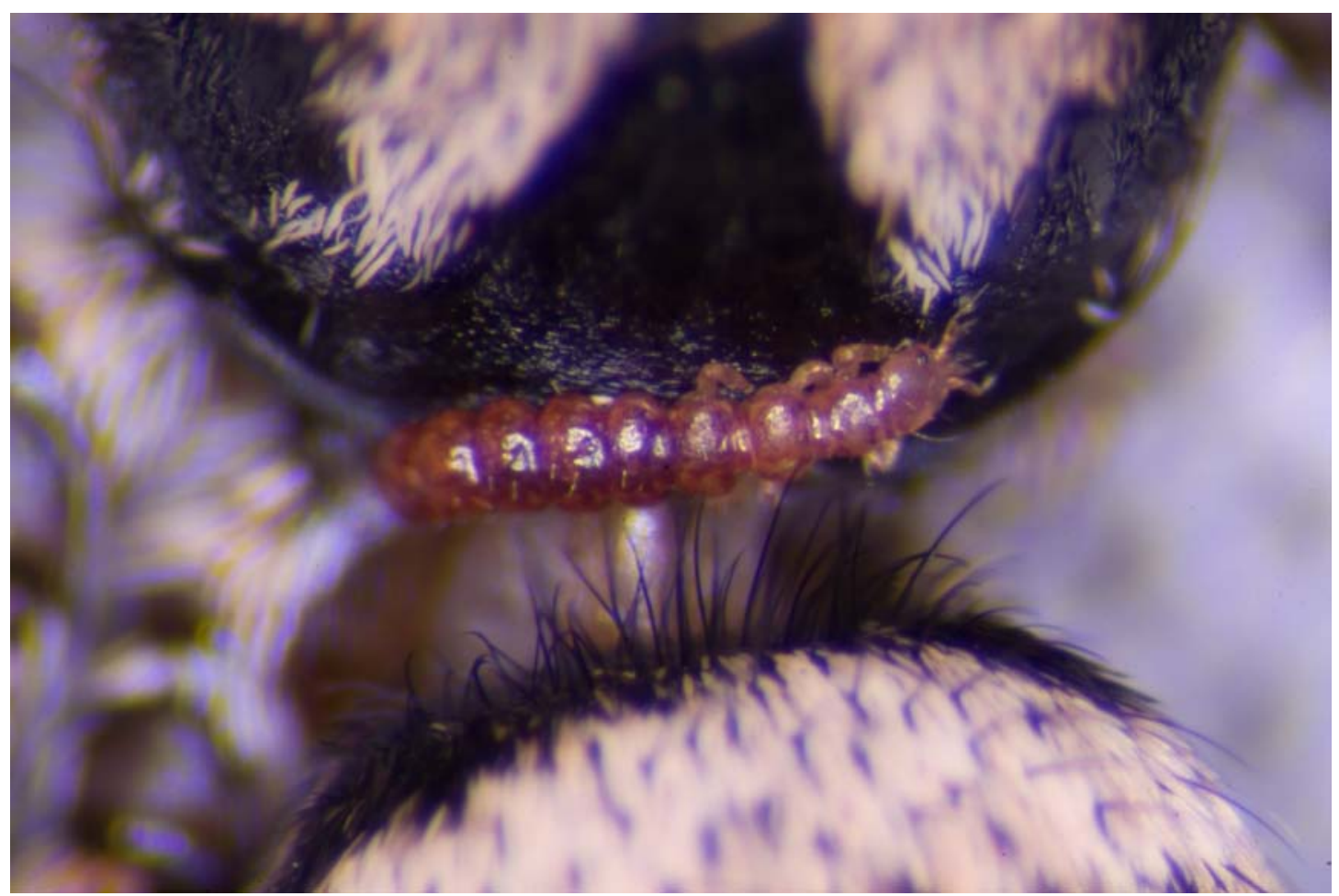

Figure 2.37. Louse attached to posterior margin of cephalothorax of juvenile $H$. oregonensis.

Mating Trials

Despite the attention and preliminary courtship gestures of all four males, the female rejected all advances.

\section{DISCUSSION}

At first glance it would seem that this species of spider is fairly eurythermic with an activity range from 17 to $34^{\circ} \mathrm{C}$. However, air temperature is not necessarily tightly correlated with substrate surface temperature, the latter being a much more relevant factor to small ground-dwelling organisms. Ground temperature was a more accurate gauge of likely spider activity than ambient air temperature, despite 
the qualitative nature of ground temperature assessment in this study (estimating surface temperature by touch). Rocks that were warm heralded the greatest number of spider encounters - any cooler or hotter generally meant that the spiders would not be active.

Ground temperatures vary as different areas of a habitat are exposed to direct sunlight; the steeply inclined habitat where $H$. oregonensis is found can be particularly variable, depending on which slope is facing the sun at different times of day. This means that the air temperature may still be relatively cool while rocks that are fully exposed to sunlight are already too hot for the spiders. The statistically significant difference in air temperature at time of collection between Mt. Hood population spiders and spiders of all other populations may point to a biologically important difference in optimal temperature ranges between the groups, though the lack of quantitative data on substrate temperatures means that these results may be misleading. The fact that spiders were encountered during nearly all daylight hours but only on substrates of a fairly consistent temperature may mean that there is not an optimal temporal range for the spiders so much as an optimal temperature range. Although I generally found spiders in the open, they were almost always associated with some amount of vegetation, even if it was rather sparse. While the spiders' interactions with this habitat component seemed minimal during the collection period, the vegetation could perhaps be important at other life stages or at 
other times of the year, perhaps providing refugia to juveniles. The vegetation may also support some of the invertebrate community upon which the spiders feed.

The spiders' apparent preference for rocks may simply be due to rocks generally being the dominant component of their habitat. Alternatively, it may be that rocks are preferentially sought out as display areas for courtship.

The small proportion of juveniles I encountered does not suggest a demographic bias, but rather implies a spatial or temporal partitioning between adults and juveniles. It may be the case that the collection sites in this study were merely display arenas for sexually mature individuals to see and be seen by the opposite sex; the rest of their lives may be spent in a different habitat entirely. This is especially relevant considering that the vast proportion of their lives would seem to be spent in the juvenile form; juveniles collected in the summer of 2009 did not molt to adulthood until the following spring or summer, oftentimes remaining in the same instar for months at a time. Admittedly, laboratory rearing conditions are a poor proxy for precise estimations of in vivo development, though the broad conclusion that juveniles will overwinter at least once seems a safe conclusion.

Males were more prevalent at all sites probably because they were out searching for females - females likely didn't need to be out for long before they found an acceptable mate. The fact that most females I encountered were not noticeably gravid may indicate that once females have mated they avoid the exposed 
habitat of the collection sites, perhaps seeking alternate, safer habitats to hunt and to lay their eggs.

While the mating trials experiment was largely unsuccessful, these trials did demonstrate that males of the Siskiyou and Tillamook populations (beige and bronze color morphs, respectively) would display to a Gorge population female (beige color morph). 
CHAPTER 3: Sexual Dichromatism and Crypsis

\section{CHAPTER OVERVIEW}

Habronattus oregonensis is a sexual dichromatic species of jumping spider that spends a portion of its life in open habitat exposed to visual predators. The degree of crypsis (particularly on the dorsum) likely has a direct influence on predator avoidance, and is thus a critical aspect of survivorship. In order to solicit mating opportunities, males present their anterior region to the females and initiate a courtship display; they are presumably judged on the quality of their performance as well as their overall appearance (S. Masta, pers. comm.). Females are presumed to be the more cryptic of the sexes, as their role in the courtship ritual is passive and not subject to evaluation by males.

Here I investigate how sexual selection may have interacted with natural selection to shape male coloration in $\mathrm{H}$. oregonensis. Using reflectance spectrophotometry and the segment classification analysis (Endler, 1990) I compare the color contrast of both sexes of four populations of this species with their respective habitats, addressing the hypotheses that natural selection has caused dorsal body regions of both sexes to be cryptic and that sexual selection has caused the anterior region of males to be less cryptic than that of females. Field work for this study was conducted in Oregon and Washington in June and July of 2009. 
Individuals from the Gorge and Siskiyou populations were nearly identical in their overall colorations. Males of these two populations were largely cryptic on the dorsal surface whereas females from these populations surprisingly had lower crypsis on their dorsum. Gorge and Siskiyou population males had much lower crypsis on their anterior compared to females. Individuals from the Mt. Hood and Tillamook populations were nearly identical in their overall colorations, but were distinctly different from those of the Gorge and Siskiyou populations. Mt. Hood and Tillamook population male dorsal surfaces were lower in crypsis than those of females; both male and female Mt. Hood and Tillamook population spiders had similar, relatively high degrees of anterior crypsis.

The lower-than-expected levels of female crypsis suggests that the sites where the spiders were collected were not the spiders' primary habitats, but rather temporary mating arenas; females (and juveniles, which have the same coloration as females) likely spend most of their lives in different habitats altogether. The large differences in magnitude and physical location of male deviations from crypsis between the two color morphs encountered suggest that there may be a difference between the morphs in the relative importance of male coloration in mate selection. Interestingly, all measured deviations of male crypsis were in the same colorimetric direction (relatively more UV/less green); this suggests that there may be a female sensory bias in place causing sexual selection to draw male coloration on a trajectory at odds with the colors being selected for by natural selection. 


\section{INTRODUCTION}

A study of visual signals requires a basic understanding of the anatomy of a signal, its transmission, its reception, and its perception (Endler, 1990; Montgomerie, 2006). The word "color" commonly refers to three basic visual aspects: hue, brightness, and chroma (Endler, 1990; Montgomerie, 2006). Hue describes the shape of a curve on a Cartesian plot wherein the x-axis is the wavelength of light and the $y$ axis is number of photons. Hue is the combinations of peaks, valleys, and plateaus of the curve within the particular range of wavelengths that the target organism is sensitive to. For example, for a human, an object that radiates light at $475 \mathrm{~nm}$ while radiating little light at other wavelengths would have a hue broadly described as "blue" (see Figure 3.1). Brightness describes the amount of light reflecting off of an object, and is independent of hue. Brightness is the area beneath the curve describing the hue; as demonstrated in Figure 3.1, both curves have identical hue (blue) but one is substantially brighter than the other. An example from photography would be stacking neutral density filters onto a lens: the hues through the viewfinder don't change, but the brightness decreases as more filters are added. The final term, chroma (also called "saturation"), refers to the steepness of the curve: the steeper the curve, the greater the chroma. Chroma is an aspect of hue; the more gradual the transition from one area of the curve to another, the lower the chroma will be. In the vernacular, the word "brightness" is often used to describe chroma; a more 
appropriate word might be "vividness" (see Figure 3.2 for an example). Throughout

this paper I will either refer to these three aspects individually or use the term

"color" as the sum total of hue, brightness, and chroma for a particular object.

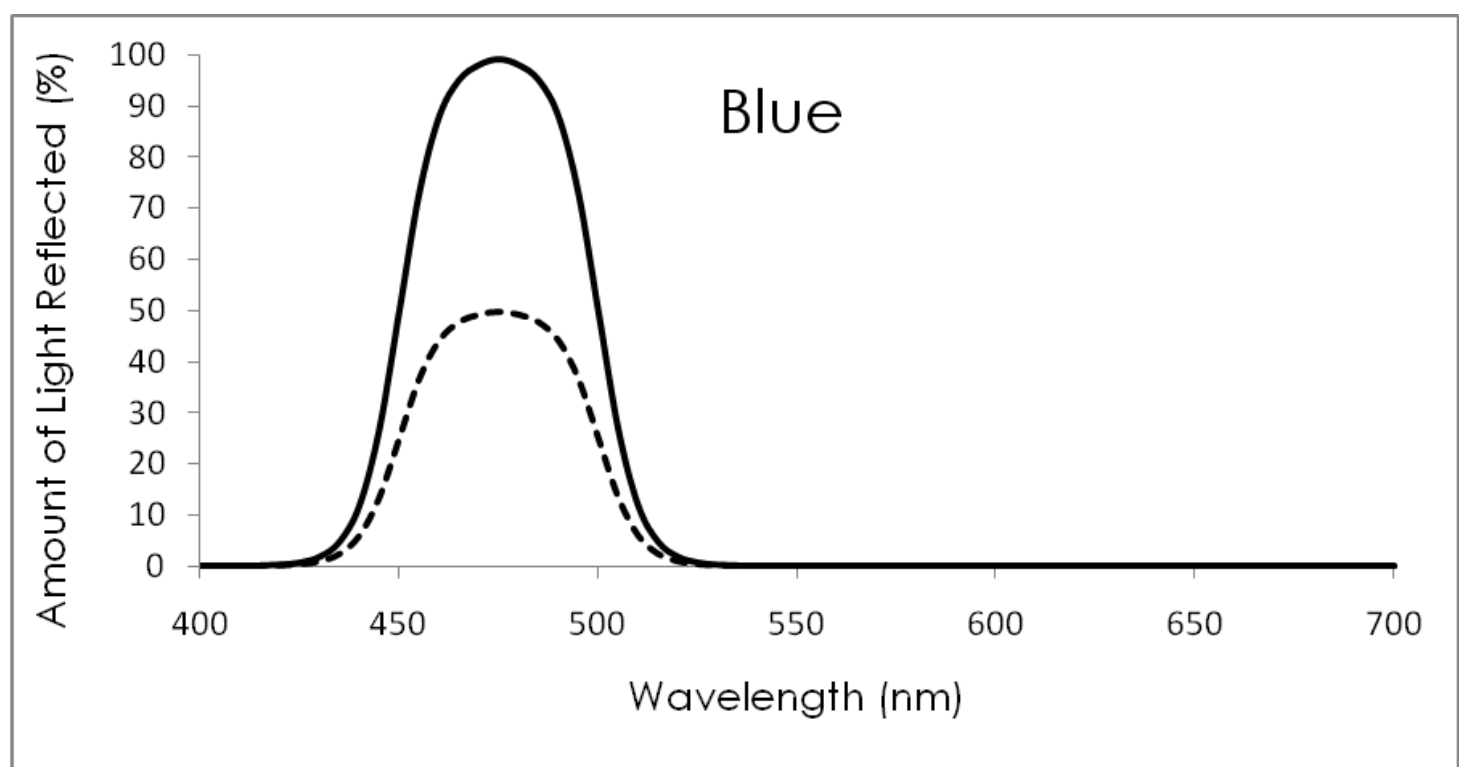

Figure 3.1. Spectral curves of two brightness levels of the same hue ("blue"). Solid curve has greater brightness, dashed curve has lesser brightness.

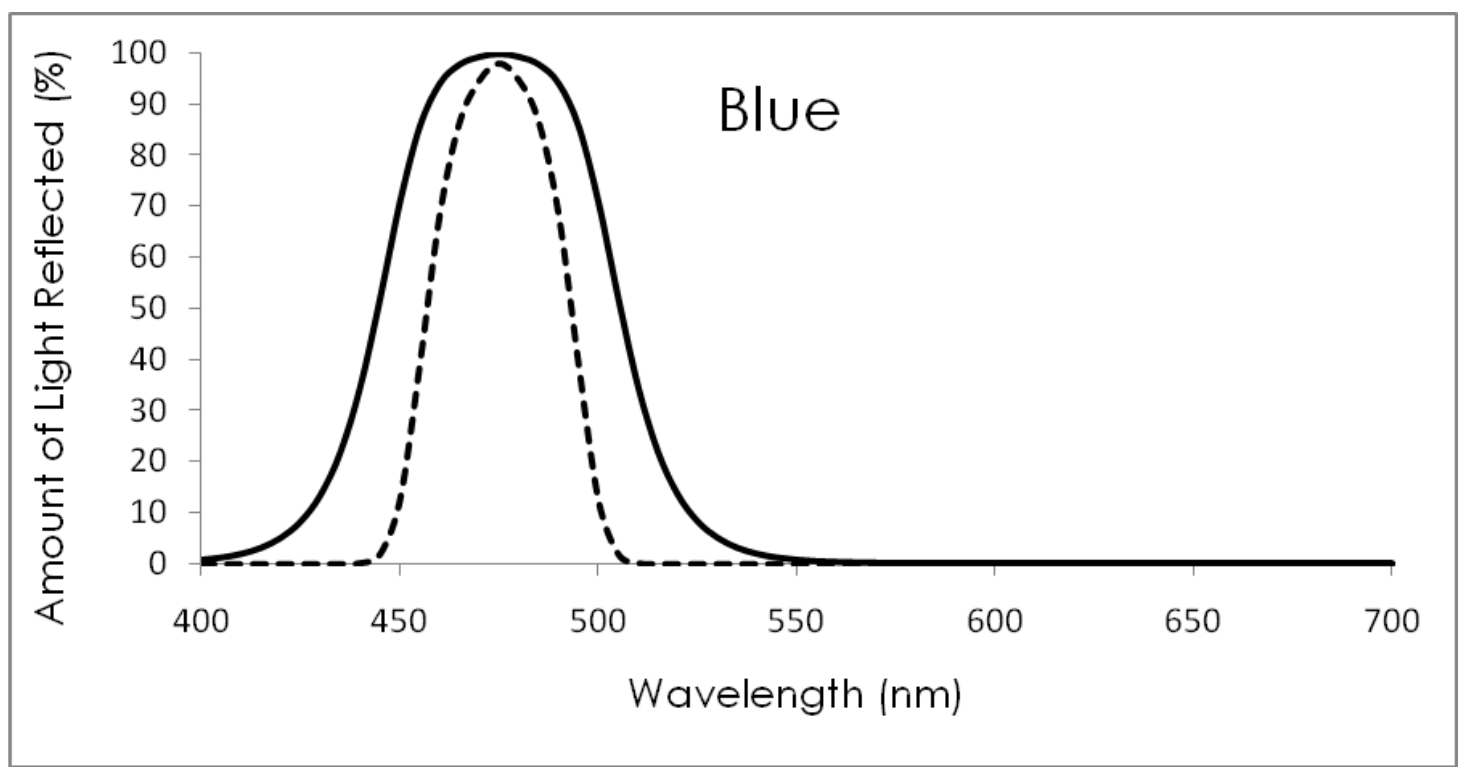

Figure 3.2. Spectral curves of two different chroma with the same peak ("blue"). Solid curve has lower chroma, dashed curve has higher chroma. 
While the reflectance properties of a particular object are unchanging, the color of the object is far from stable (Andersson and Prager, 2006; Endler, 1990). The light reflected off of that object can change dramatically depending on ambient lighting conditions; for example, an object that only reflects blue light will not appear blue when illuminated by red light. Given the potential for variation in radiance despite unchanging reflectance properties, when attempting to quantify the reflected color of an object one must take into consideration all the light radiating off of the object that makes it to the perceiving sensory system. Endler (1990) astutely divided a visual signal into three basic components: ambient light, reflected light, and transmitted light. Ambient light can have a number of components in natural settings, the simplest of which being direct irradiance from the sun. The spectral qualities of daylight are not constant, however, and can vary depending upon the time of day, season, and location on the globe. Ambient light is also affected as it is transmitted through various media; anything from clouds to tree leaves in a closedcanopy forest (Endler, 1993). Finally, ambient light is also composed of light reflected off of nearby objects (Endler, 1993).

Reflected light radiating off of an object is a combination of all the ambient light that the object is exposed to minus the component of light that is absorbed (as dictated by the reflectance properties of the object). Transmitted light is the portion of the reflectance spectra radiated off of the target object that is not absorbed by the media through which it must travel in order to reach the receiving visual system. The 
amount of light absorbed by clean air at relatively short distances is, for most intents and purposes, negligible, though dust and fog in air as well as sediment and dissolved organic matter in water can have considerable effects on the transmission of a signal (Endler, 1991).

Finally, one must consider the visual system receiving the signal. The range of wavelengths that the visual pigments are sensitive to are obvious limiters to what can be detected, though such sensitivities are not necessarily a precise representation of biological relevance - factors such as lens and cornea transmission spectra (for example, humans cut off at approximately $400 \mathrm{~nm}$ and birds tend to cut off at approximately $320 \mathrm{~nm}$, despite visual pigment sensitivities in both cases that extend below these ranges (Andersson et al., 1998; Goldsmith, 1990; Montgomerie, 2006)), peak versus sub-optimal wavelength sensitivity of the visual pigments, and proximity of the visual pigment sensitivity peaks to each other all contribute to the final interpretation of the signal, which is ultimately acted upon (or ignored) by the receiving individual. It's worth mentioning here that, until the last couple of decades, most vision-related studies were by necessity strongly biased toward the human range of perception (Endler, 1990). With a few exceptions, before spectrophotometers were widely available and relatively affordable, human vision was used as a proxy in studies of crypsis (Andersson and Prager, 2006; Endler, 1990). This oftentimes resulted in severe compromises in the applicability of conclusions derived from these studies, depending on the organisms under scrutiny. There are 
several reasons to interpret these studies with caution. First of all, human color vision is sensitive to wavelengths between about $400 \mathrm{~nm}$ and $700 \mathrm{~nm}$; this is somewhat unusual compared to most other visual organisms in that human sensitivity is shifted towards longer wavelengths (Andersson and Prager, 2006). Ranges of $320 \mathrm{~nm}$ to $650 \mathrm{~nm}$ or so is far more typical, a trend that is common in such disparate animals as bird and insects (Andersson and Prager, 2006; Briscoe and Chittka, 2001; Goldsmith, 1990; Peitsch et al., 1992). Human vision is also quite notable in its emphasis of and sensitivity to the particularly small range of wavelengths between about $530 \mathrm{~nm}$ and $555 \mathrm{~nm}$; this is a result of having the peak wavelength sensitivity of two of our three visual pigments very close to each other (Merbs and Nathans, 1992). Subtle differences in this range are particularly apparent to humans with normal color vision, but would likely go unnoticed in organisms without such specialization. Of course, such a phenomenon can manifest in other organisms as well - any organism with two visual pigment sensitivity peaks that are comparatively closer together will experience a greater ability to discriminate between wavelengths in that range.

In organisms with two or more visual pigments, color perception is often defined by a system of opponency between alternating pairs of such pigments, rather than adjacent pairs (Endler, 1990; Menzel and Backhaus, 1989). This system is known to occur in such diverse taxa as humans and honeybees, and I make the assumption that it applies to all taxa investigated in this study. Making use of this relatively 
constant mechanism, Endler (1990) developed a generally applicable method of visualizing color in a generalized color space called "segment classification" (see materials and methods for details) - this method is extremely useful for displaying color data when the precise details of the target organism's vision are unknown.

While human color vision may very well be a reasonable approximation to a different visual system under scrutiny, potential differences must not be ignored. Familiarity with the vision system of the target organism is essential if one is to make biologically relevant conclusions. Unfortunately, detailed vision system studies are rare, with birds (Cuthill et al., 1999; Endler and Mielke, 2005; Hart, 2001; Hart and Hunt, 2007; Hart and Vorobyev, 2005) and honeybees (Backhaus, 1991; Backhaus and Menzel, 1987; Chittka, 1992; Chittka et al., 1992) being the two main nonhuman taxa with any sort of in-depth data available. However, with the knowledge of a few basic factors of the organism's visual psychophysiology, a reasonable approximation of the vision system can be modeled. Range of wavelength sensitivities and an estimation of peak wavelength sensitivities are the most fundamental; an assumption of an opponency system is important if presenting the data using the segment classification method. In studies of crypsis where aspects of the vision system are unknown and estimations must be made, gaps in knowledge of the vision system must be stated upfront and taken into consideration when reviewing the study's conclusions. 
Crypsis is the degree to which the coloration of an animal matches a random patch of its environment (Endler, 1983, 1990, 1993; Endler and Théry, 1996). The less it matches, the more conspicuous it is. It is important to note, however, that the degree of crypsis is entirely dependent upon the perceiving vision system. For example, an organism may have bright red patches that bear no resemblance whatsoever to its habitat, but it may still maintain perfect crypsis when viewed by a predator that can only see in the blue range. Another important factor to bear in mind is that crypsis is not an inherent, unchanging constituent of the potentially cryptic animal and the perceiving vision system: the degree to which the coloration of the animal matches its background can change as the ambient light changes. If the aforementioned predator could see into the red range but the prey was only active in places or times when there was no ambient red light, then its crypsis would be maintained.

The efficacy of a visual signal is directly related to the contrast of that signal with its environment (background noise) and the maintenance of signal fidelity, as perceived by the receiving visual system (be it a potential mate, predator, or prey item) (Endler, 1992). Attraction of a mate and maintenance of crypsis is a balance between the not-necessarily equal visual systems of the target organisms (the potential mates, generally the female), and the intercepting organisms (potential predators and prey). Natural selection favors maximal visual signal transmission to the target and the obfuscation of this signal to all other potential recipients. When 
the visual systems overlap and the target and non-target organisms can both perceive the same signal, a compromise results, and a value must emerge that maximizes the male's success in securing the attention of mates and minimizes detection of the male's signals by all relevant non-target organisms.

A basic knowledge of the visual systems of the primary target and non-target organisms can be combined with the ambient light levels of the habitat at the appropriate time and place, the photometric characteristics of the male's coloration, and the visual background upon which these signals are superimposed to evaluate how far the male's coloration has deviated from crypsis (Endler, 1990). Deviations from cryptic coloration within the range of detection by females will imply sexually selective pressure. Assuming that the females are not under sexually selective pressure for coloration, female cryptic coloration can be used as a standard to indicate what is physiologically capable of emerging in the target taxon under the same pressure for crypsis, but without sexually selective pressure against crypsis being applied. The designation of female coloration as "more cryptic" and male coloration as "less cryptic" can be supported empirically by comparison with the color of the organism's habitat, as well as logically; Macedonia (2001) pointed out that in instances where the females and juveniles (of lizards, but broadly applicable to other taxa as well) of both sexes have identical coloration and adult males transition to an alternate coloration, the delayed transition of males implies that the 
alternate coloration is a detriment to individual survival and is only necessary for mating purposes.

Habronattus oregonensis, a visually acute and highly sexually dimorphic species of jumping spider, presents an ideal system in which to investigate the interplay between natural and sexual selection. The spiders are found in exposed habitats of open ground, rock piles, and occasionally leaf litter (personal observations) from Vancouver, British Columbia and Alberta through New Mexico and southern Arizona (S. Masta, pers. comm.; Paquin et al., 2010; Griswold, 1987; Peckham and Peckham, 1909). This exposed environment leaves the spiders vulnerable to vision-oriented predators, particularly spider-hunting wasps. Adult male $H$. oregonensis possess sexual ornaments on their anterior and have dorsal colorations that differ from that of females and sub-adults. Distinctive male colorations likely play a role in securing mating opportunities from the highly selective females (Peckham and Peckham, 1889; Peckham and Peckham, 1890; Richman, 1982); however, these traits may make males more visible to predators. These differences in coloration between males and females suggest that sexual selection may have opposed environmental pressures for crypsis among sexually mature males. Comparisons between males and females of different populations and their respective habitats make it possible to ask whether and in what manner sexual selection has interacted with natural selection to shape male morphology. 
The visibility of different body regions to predators and prey as well as potential mates may influence the relative amount of selection for crypsis; I investigate the anterior of the cephalothorax, the dorsal aspect of the cephalothorax, the dorsal aspect of the abdomen, and the ventral aspect of the abdomen.

Jumping spiders as a group are unusual among spiders in that they have excellent vision with which they use to hunt their prey (Huber, 2005). They use their anterior and posterior lateral eyes as motion-detectors to locate their target and, upon detection, orient the cephalothorax towards the target such that it is visible to the much more acute anterior median (principal) eyes (Clark and Uetz, 1990; DeVoe, 1975). It is the principal eyes that have multiple visual pigments and are responsible for color discrimination; the lateral eyes employ only one type of visual pigment (Yamashita and Tateda, 1976). Based on studies that have quantified the spectral ranges of several species of jumping spiders (Blest et al., 1981; DeVoe, 1975; Peaslee and Wilson, 1989), I estimate that $H$. oregonensis is primarily sensitive to light between $330 \mathrm{~nm}$ and $650 \mathrm{~nm}$. While it is likely that they are sensitive to light outside this range (perhaps 300-700nm), peripheral wavelengths of light are likely only dimly perceived and as such are not as biologically relevant.

Field observations that I made during this study revealed spider wasps to be the most likely primary predators of $H$. oregonensis. I found $H$. orgeonensis to be a generalist predator that feeds on any insect of the appropriate size. As insects tend to have similar spectral sensitivities to $H$. oregonensis (Peitsch et al., 1992), I use light 
wavelengths between $330 \mathrm{~nm}$ and $650 \mathrm{~nm}$ as a reasonable estimate for the most biologically relevant segment of ambient light perceived by $\mathrm{H}$. oregonensis, its predators, and its prey. Potential differences in neural processing and influences on perception based on the number of visual pigments are valid concerns, but out of the scope of this paper.

There are two alternative hypotheses tested here:

1. Natural selection will direct the coloration of body regions visible to predators to be convergent with the coloration of the substrate upon which the spiders are found. The null hypothesis is there will be no correlation between coloration of exposed body regions and habitat coloration.

a. As the likely principal predators of $H$. oregonensis fly, the body region of the spiders most likely to be detected by predators will be the dorsal surfaces of the cephalothorax and abdomen.

b. Regardless of the actual coloration of the different populations of $H$. oregonensis, the relative differences in color between the spiders and their respective habitats will be similar among the populations due to similar levels of predation pressure.

2. Sexual selection will drive male spiders to be less cryptic than female spiders in body regions presented during courtship, due to selection for courtship colorations in males but not females. The null hypothesis is there will be no 
difference in degree of crypsis of body regions presented during courtship between male and female spiders.

a. Deviation from crypsis among males will be concentrated on the anterior of the cephalothorax, as this is the primary region of the body that is presented to females during courtship. Females may deviate from crypsis in the anterior, depending on gene linkage and the intensity of natural selection acting on the anterior.

\section{MATERIALS AND METHODS}

Specimens and Habitat Samples

I collected spiders from four different populations; I gathered representative fragments of the substrate upon which each spider was first encountered for subsequent color analysis (See chapter 2 for additional field data collected and for details of each collection site). The names I gave to the four populations addressed in this study are the following: Gorge population, Siskiyou population, Mt. Hood population, and Tillamook population.

\section{Specimen Preparation}

In addition to 65 individuals caught as adults, a total of five juveniles that were raised to adulthood were included in this analysis; however, because juvenile habitat may differ in coloration from adult habitat, the habitat samples associated 
with these five individuals were omitted from analysis. Specimens were euthanized and preserved by freezing at $-80^{\circ} \mathrm{C}$. When ready for analysis each specimen was thawed and photographed for subsequent morphometric analysis (see chapter 4) and measured with a spectrophotometer for color analysis.

Spectrophotometry

I made all measurements of reflectance spectra with an Ocean Optics USB4000 Miniature Fiber Optic Spectrometer and a Mikropack DH-2000 DeuteriumHalogen Light Source. Reflectance measurements were calibrated with a Labsphere WS-1-SL diffuse white reflectance standard with Spectralon. All measurements were taken in the Ocean Optics software program SpectraSuite. All reflectance measurements were taken in a darkened room to minimize stray light contamination. The reflectance probe was held perpendicular (coincident normal) to the surface of the subject. All readings were taken at a distance of $2 \mathrm{~mm}$ from the target surface (cf. Lim and Li $(2006,2007)$ ). Each measurement was repeated five times (cf. Lim and Li $(2006,2007))$, haphazardly scanning different locations of the target region.

I selected four body regions of the spiders for spectrometer analysis: anterior of the cephalothorax (the face), dorsum (cephalothorax), dorsum (abdomen), and ventrum (abdomen). For anterior measurements, palps were not included due to difficulty in standardization of measurements. The entire face of the spider (including the clypeus and cheliceral setae) was included in the five replicate 
measurements, excluding the eyes. Fragments of habitat associated with each spider were separated into groups of like objects (rocks, dry leaves, dry grass, etc.) and were measured using the same techniques mentioned above.

I reduced data into $10 \mathrm{~nm}$ bins during processing for ease of calculation (c.f. Macedonia (2001) and Eaton (2005)). The median of the data points in each bin was taken rather than the mean in order to reduce the influence of noise and artifactual spikes in the data (such as the D-alpha line) (c.f. Cuthill et al. (1999), Macedonia (2003), and Montgomerie (2006)).

\section{Solar Irradiance Data}

I made all collections of $H$. oregonensis during the summer (between 16 June and 21 July) with clear skies and no canopy cover. This homogeneous light regime allowed me to use solar irradiance values collected by the National Renewable Energy Laboratory's Solar Radiation Research Laboratory Baseline Measurement System as a reasonable stand-in (Solar Radiation Research Laboratory (BMS), 2010). I used data from days that matched this light regime from their archive of irradiance data taken with a LI-COR LI-1800 Spectroradiometer with the sensor oriented at Global Horizontal (available between 13 June 2006 and 10 July 2008). These readings were taken in Golden, Colorado (latitude $39.74^{\circ} \mathrm{N}$, longitude $105.18^{\circ} \mathrm{W}$, elevation $1829 \mathrm{~m})$. I chose to use irradiance data from the time of day at which I encountered the most $H$. oregonensis: 1400 hours. Slight differences may exist between the NREL 
data and the actual solar irradiance spectra present at the time of collection:

variations in abundance of particulate matter and aerosol droplets in the atmosphere leading to different amounts of Mie scattering would be the most likely differences (Hyslop, 2009; Smith, 1982). Mie scattering is caused by particulate matter or aerosol droplets with diameters larger than the target wavelength of light. These particles or droplets scatter light at wavelengths smaller than their diameter - the net effect would be a roughly uniform scattering of light at all wavelengths analyzed here. While this may have an effect on light intensity, the more important relative proportions of wavelengths will remain unchanged - therefore leaving my conclusions unaffected. Rayleigh scattering, the processes whereby shorter wavelengths of light are scattered by gas and small particles, would impart small changes in the availability of UV, violet, and blue at different elevations. However, these changes would be relatively small compared to that of Mie scattering (Hyslop, 2009). Finally, it is unlikely to generate spurious color matches between spider and habitat due to slight variations in solar irradiance because if the spiders have the same reflectance properties as their habitat (i.e., cryptic), then the coloration of the spiders will vary with the coloration of their habitat, regardless of the ambient light. While field measurements of solar irradiance would be preferable, the NREL database is a reasonable substitute.

Segment Classification Analysis 
I followed Endler's (1990) model for the construction of a generalized color space. This method operates under the assumption that the standard method of color processing in invertebrates (and vertebrates) is by comparing relative ratios of nonadjacent paired segments of the color spectrum (Endler, 1990; Hurvich, 1981). I analyzed the reflectance data combined with solar irradiance values collected by the National Renewable Energy Laboratory's Solar Radiation Research Laboratory Baseline Measurement System (Solar Radiation Research Laboratory (BMS), 2010). I tailored the analysis to include light in the range of $330 \mathrm{~nm}$ to $650 \mathrm{~nm}$, dividing this range into four equal segments: Quadrant 1 (Q1): 330-410nm, Quadrant 2 (Q2): 410490nm, Quadrant 3 (Q3): 490-570nm, and Quadrant 4 (Q4): 570-650nm.

The first step of this analysis is to determine the area under the spectral curve within each of these four segments. This is accomplished by the following equation:

$$
Q_{\mathrm{T}}(x) \equiv \sum Q(\lambda, x)
$$

where brightness values within the target region are summed (Endler, 1990). The relative amount of brightness of each segment is calculated by dividing the brightness of a single segment by the brightness of the entire spectrum. The segments are then divided into "opponents" by subtraction of Q1 from Q3 and Q2 from Q4. This leaves two values from which to calculate both hue and chroma. Chroma is calculated with the following equation:

$$
C=\sqrt{(\mathrm{Q} 3-\mathrm{Q} 1)^{2}+(\mathrm{Q} 4-\mathrm{Q} 2)^{2}}
$$


This equation generates a value for chroma represented by the Euclidean distance from the origin of a polar plot of the color space (Endler, 1990). To calculate hue, the following equation is used:

$$
H=\operatorname{arcSin}\left(\frac{\mathrm{Q} 3-\mathrm{Q} 1}{C}\right)=\arccos \left(\frac{\mathrm{Q} 4-\mathrm{Q} 2}{C}\right)
$$

The values representing hue generated by this equation correspond to the angle on a polar plot of the color space clockwise from Q4 (the 12 o'clock position on the plot) (Endler, 1990).

The hue and chroma values of a spectrum are then plotted on a polar scatter plot. The greater the Euclidean distance between two points, the greater the disparity in the hue and chroma components of color. Note that brightness is automatically factored out of this analysis and must be addressed separately.

\section{Statistical Analysis}

The benefit of plotting spectral data onto a color space is that major trends in the relationships among spectra are lucidly rendered, though it does not imply significance. Despite this shortcoming, it is the best approximation for visual systems where the necessary supporting neurophysiological data of the target taxon is absent. The calculation of $t$-tests of the differences between the hue and chroma of different groups of objects provides numerical support to the trends visible in the color space plots and draws attention to trends that may be less obvious. Brightness values can also be examined separately from hue and chroma with $t$-tests. 
The difference between the hue and chroma of two different spectra can be calculated with the following equation:

$$
D_{H C}=\sqrt{\left(H_{\mathrm{S} 1}-H_{\mathrm{S} 2}\right)^{2}+\left(C_{\mathrm{S} 1}-C_{\mathrm{S} 2}\right)^{2}}
$$

Where S1 is subject 1 and S2 is subject 2 . This is modified from the original equation by Endler (1990) to exclude the brightness component; I found that because brightness is in a different scale from that of hue and chroma, running a $t$-test on values generated from an equation inclusive of all three factors may be misleading.

The difference between the brightness of two different spectra can be calculated with the following equation:

$$
D_{B}=\frac{B_{\mathrm{S} 1}-B_{\mathrm{S} 2}}{B_{\mathrm{S} 1}+B_{\mathrm{S} 2}}
$$

Where S1 is subject 1 and S2 is subject 2 (Fleishman and Persons, 2001; Leal and Fleishman, 2004; Macedonia et al., 2004; Stuart-Fox et al., 2007; Uy and Endler, 2004). Schultz (2001), LeBas and Marshall (2000), Andersson et al. (1998), and Endler and Théry (1996) also use a similar version of this equation to calculate difference in brightness. This equation results in a value between -1 and 1 . When the value approaches 1 , subject 1 is much brighter than subject 2 ; the closer the value is to -1 , the brighter subject 2 is in comparison to subject 1 . If the value is close to zero, the level of brightness between the two subjects is similar.

I ran $t$-tests on both the raw values of brightness and hue+chroma, as well as the contrast values of the spiders against their habitat. 


\section{RESULTS}

Solar Irradiance Spectra

Figure 3.3 is a graphical representation of the solar irradiance spectra used in this study (Solar Radiation Research Laboratory (BMS), 2010). These data were used to adjust the reflectance measurements of spiders and their habitat to in situ spectral values. Note that shorter wavelengths are less well-represented than longer wavelengths; this means that, all other things being equal, objects reflective of shorter wavelengths will be less apparent that objects reflective of longer wavelengths. This inequality of light availability is one reason why solar irradiance must be considered in vision studies such as this. 


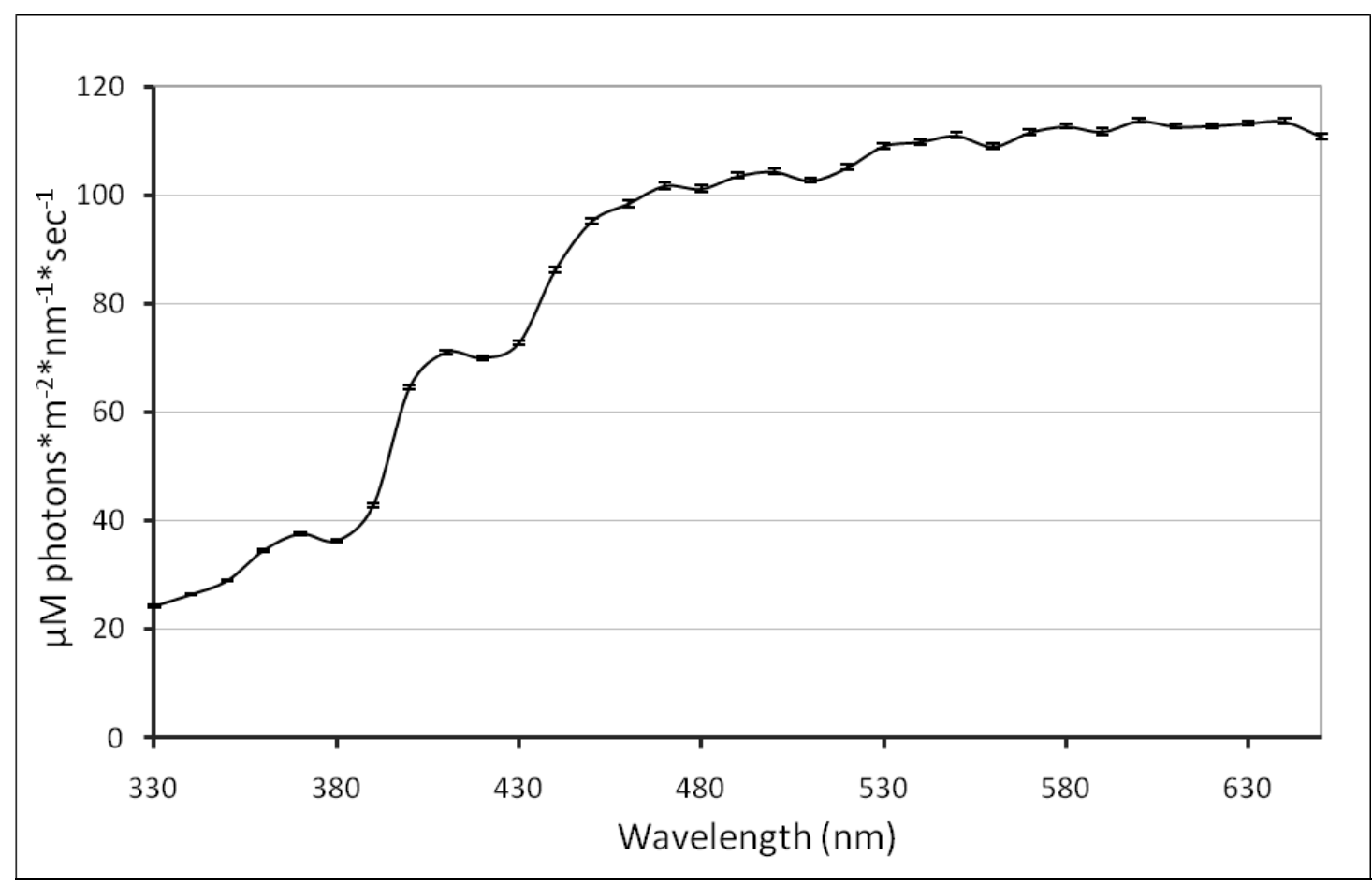

Figure 3.3. Solar irradiance spectra. Mean of seven sunny days between 13 June and 27 July at 1400 hours. Readings taken at Golden, CO (latitude $39.74^{\circ} \mathrm{N}$, longitude $105.18^{\circ} \mathrm{W}$, elevation $1829 \mathrm{~m}$ ) (Solar Radiation Research Laboratory (BMS), 2010). Error bars are standard error.

\section{Reflectance Spectra}

Figures 3.4 through 3.13 are the mean spectra of males and females of Gorge and Mt. Hood populations. Reflectance spectra of individuals of the Gorge population are similar to those of the Siskiyou population (collectively, the "beige" morph) and reflectance spectra of individuals of the Mt. Hood population are similar to those of the Tillamook population (collectively, the "bronze" morph). These figures highlight differences in reflectance between males and females of the different color morphs at all wavelengths visible to the spiders and their predators 
and prey. The figures provide a preliminary glimpse of the differences in coloration between the sexes, and potential differences in their degree of crypsis.

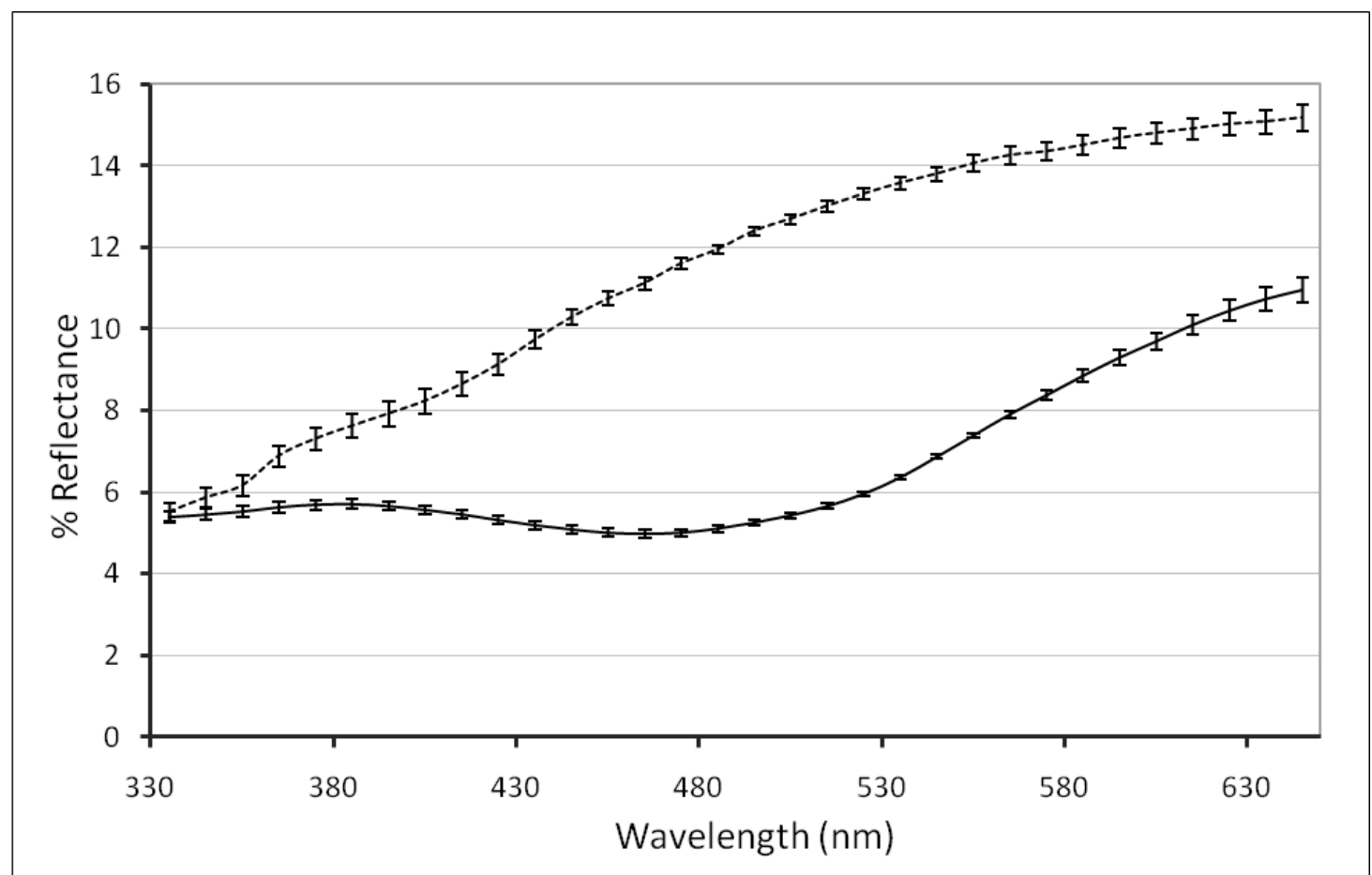

Figure 3.4. Gorge population reflectance: beige morph face. Solid line $=$ male, dashed line $=$ female. Equalized for brightness, error bars are standard error of hue. Male $n=14$, female $n=7$. 


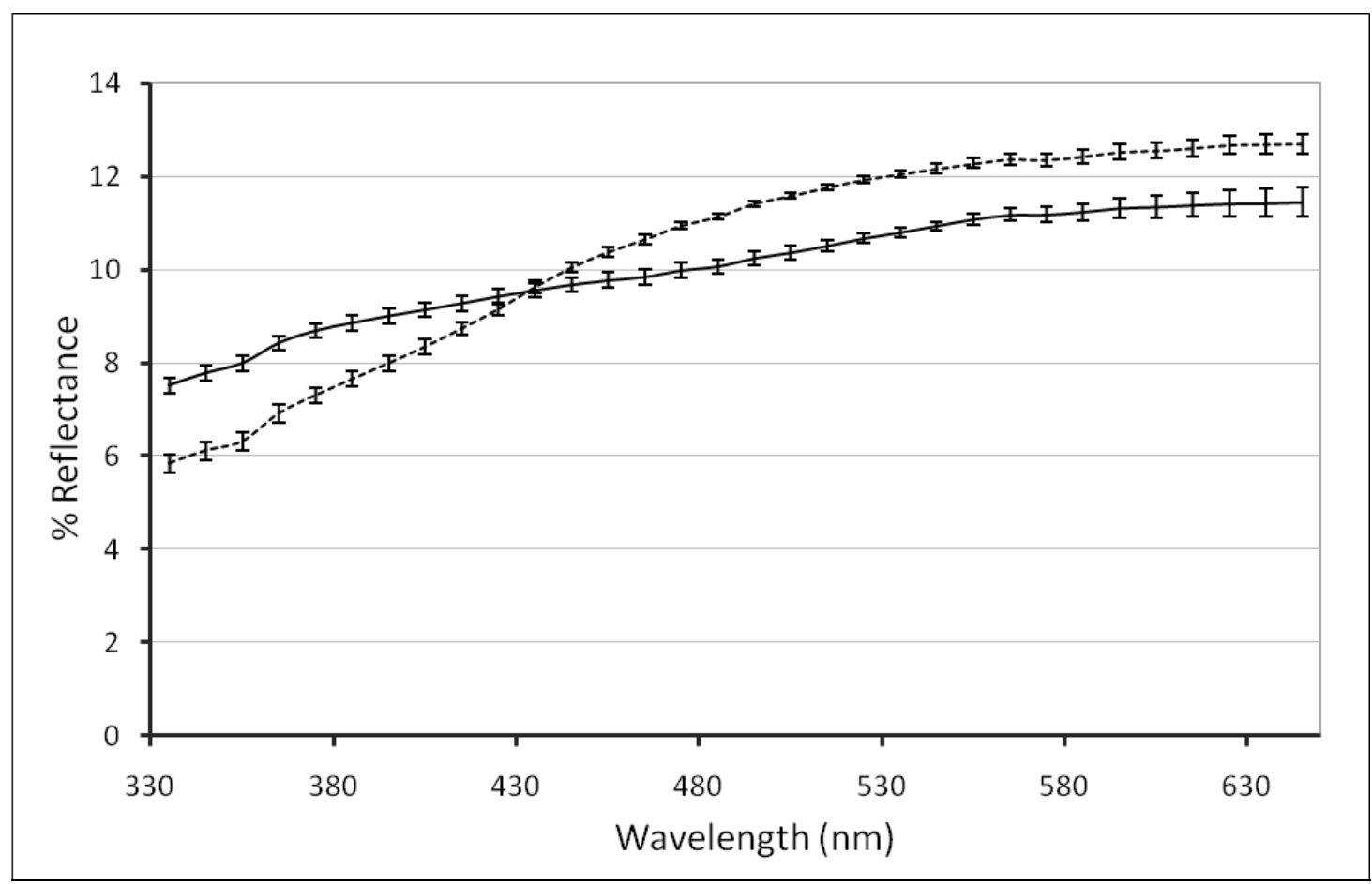

Figure 3.5. Mt. Hood population reflectance: bronze morph face. Solid line $=$ male, dashed line $=$ female. Equalized for brightness, error bars are standard error of hue. Male $n=9$, female $n=7$.

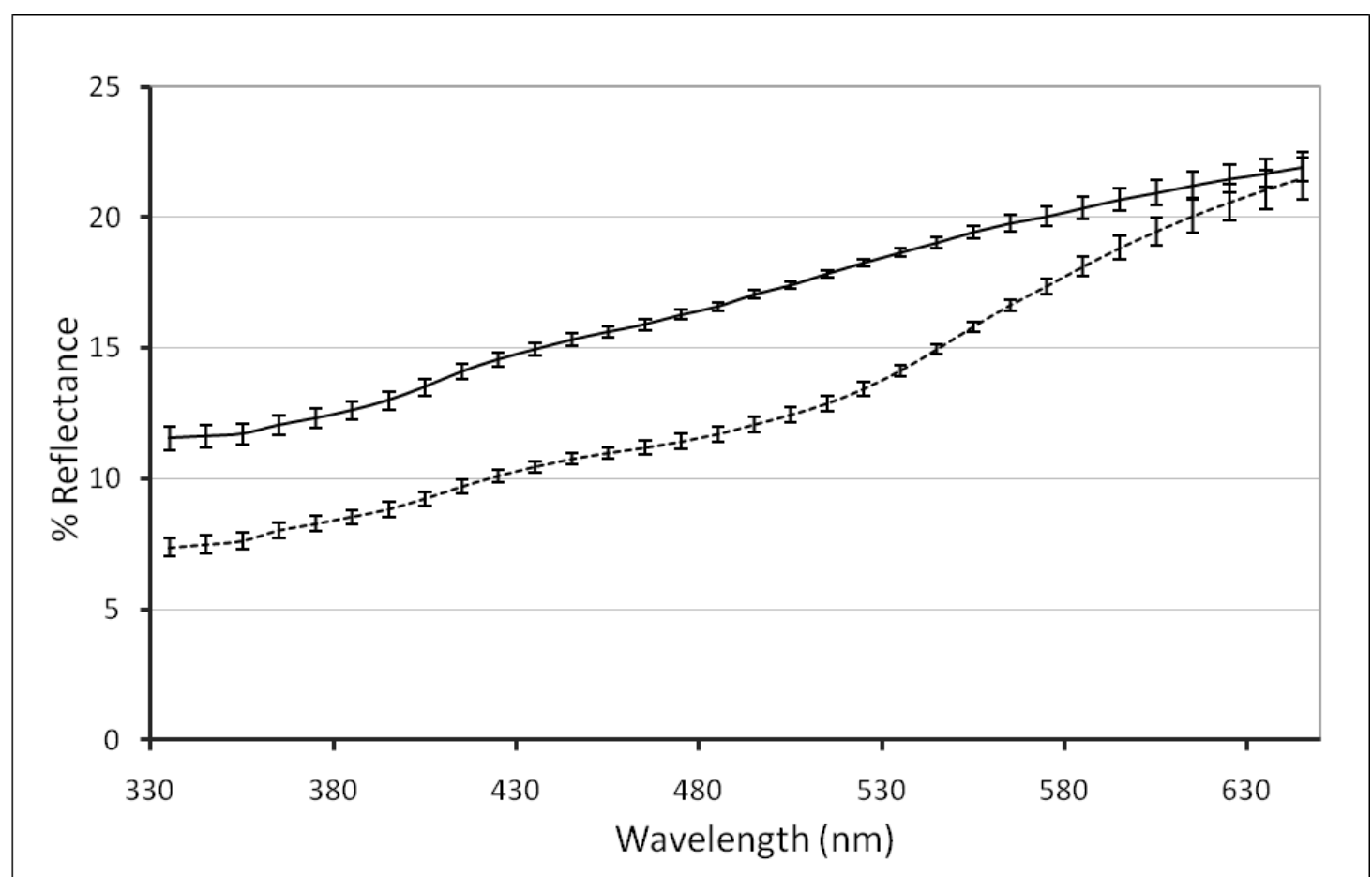

Figure 3.6. Gorge population reflectance: beige morph cephalothorax. Solid line = male, dashed line = female. Equalized for brightness, error bars are standard error of hue. Male $n=9$, female $n=7$. 


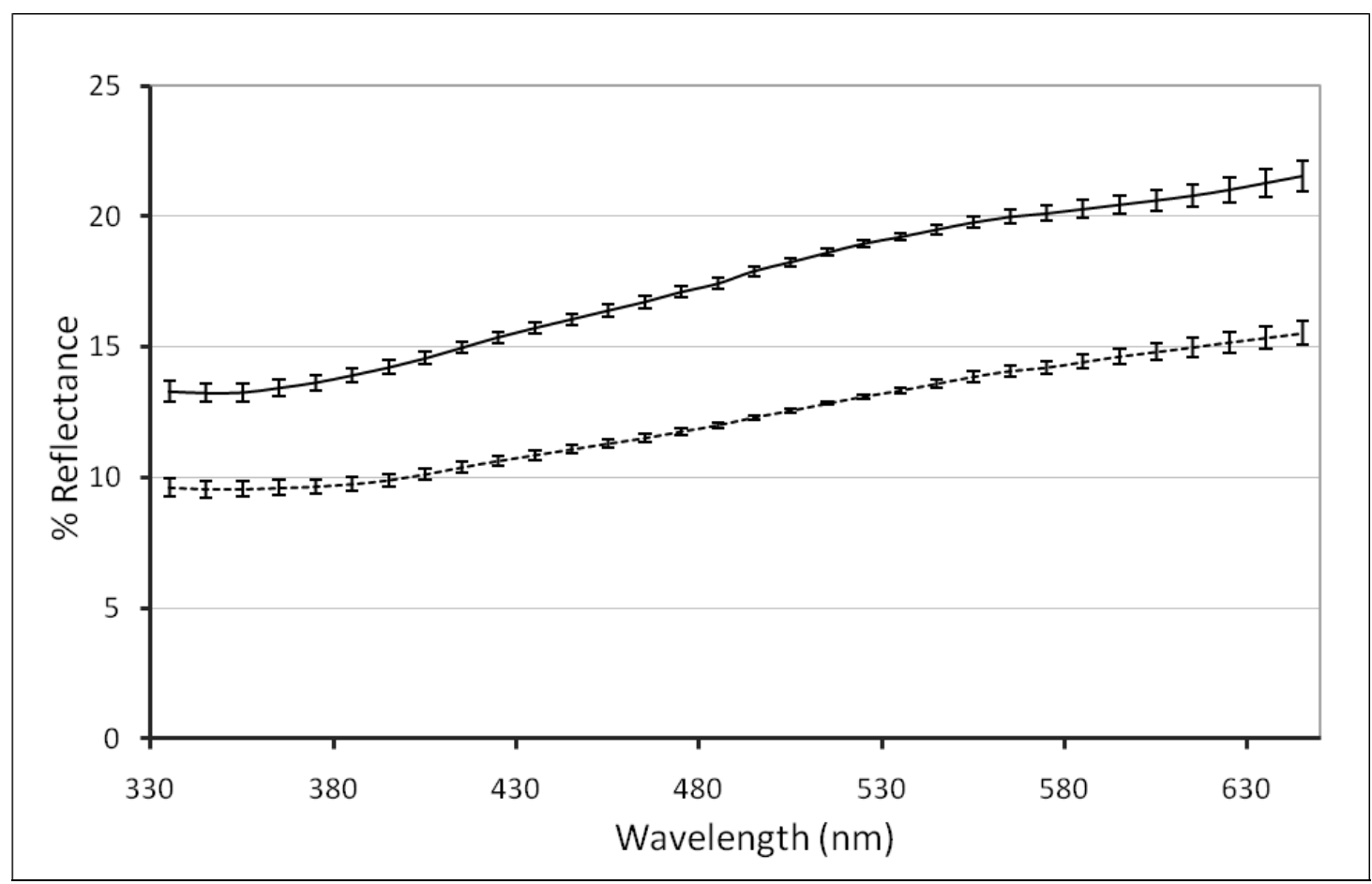

Figure 3.7. Mt. Hood population reflectance: bronze morph cephalothorax. Solid line $=$ male, dashed line $=$ female. Equalized for brightness, error bars are standard error of hue. Male $n=9$, female $n=7$.

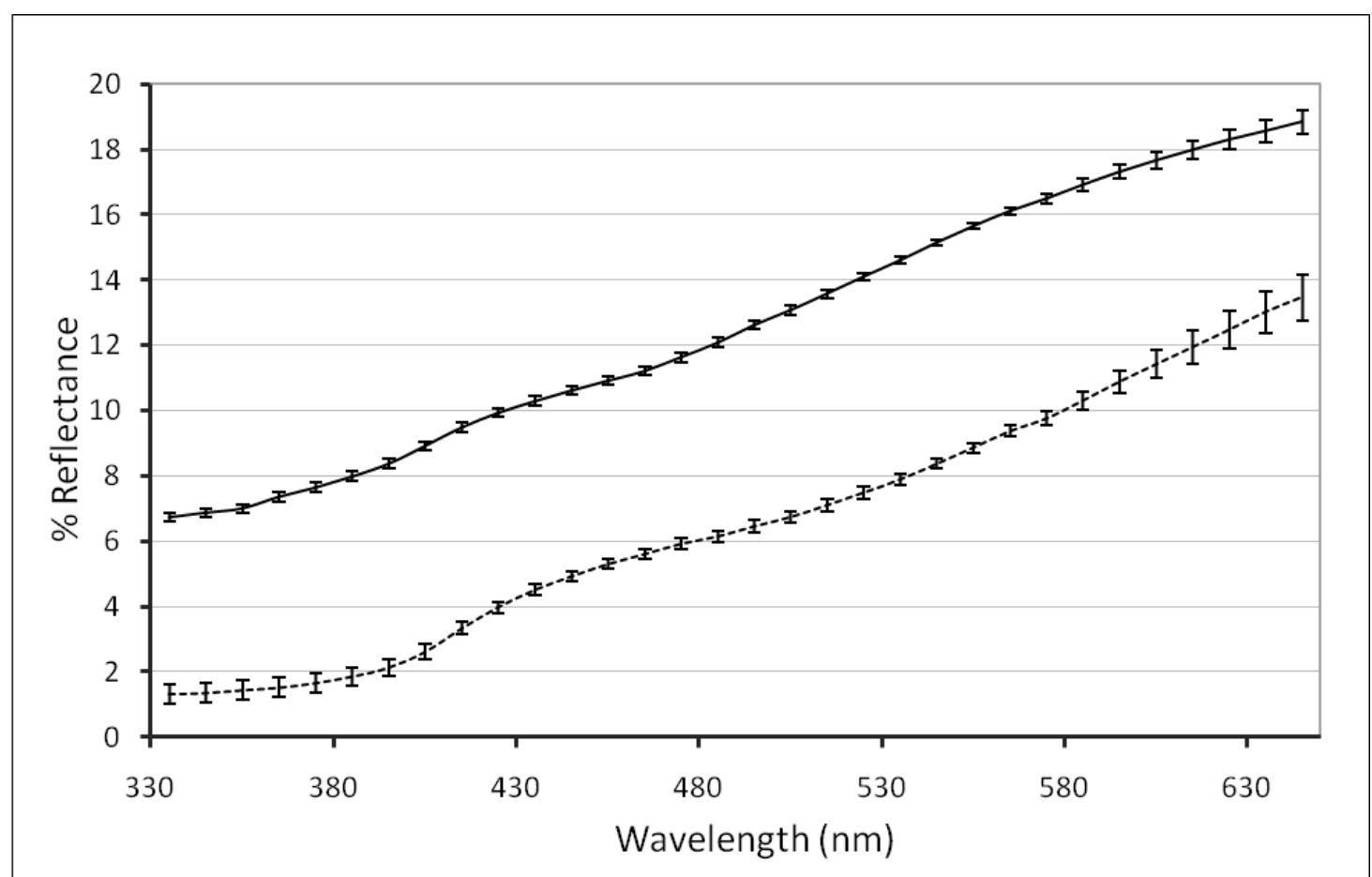

Figure 3.8. Gorge population reflectance: beige morph abdomen. Solid line $=$ male, dashed line $=$ female. Equalized for brightness, error bars are standard error of hue. Male $n=13$, female $n=7$. 


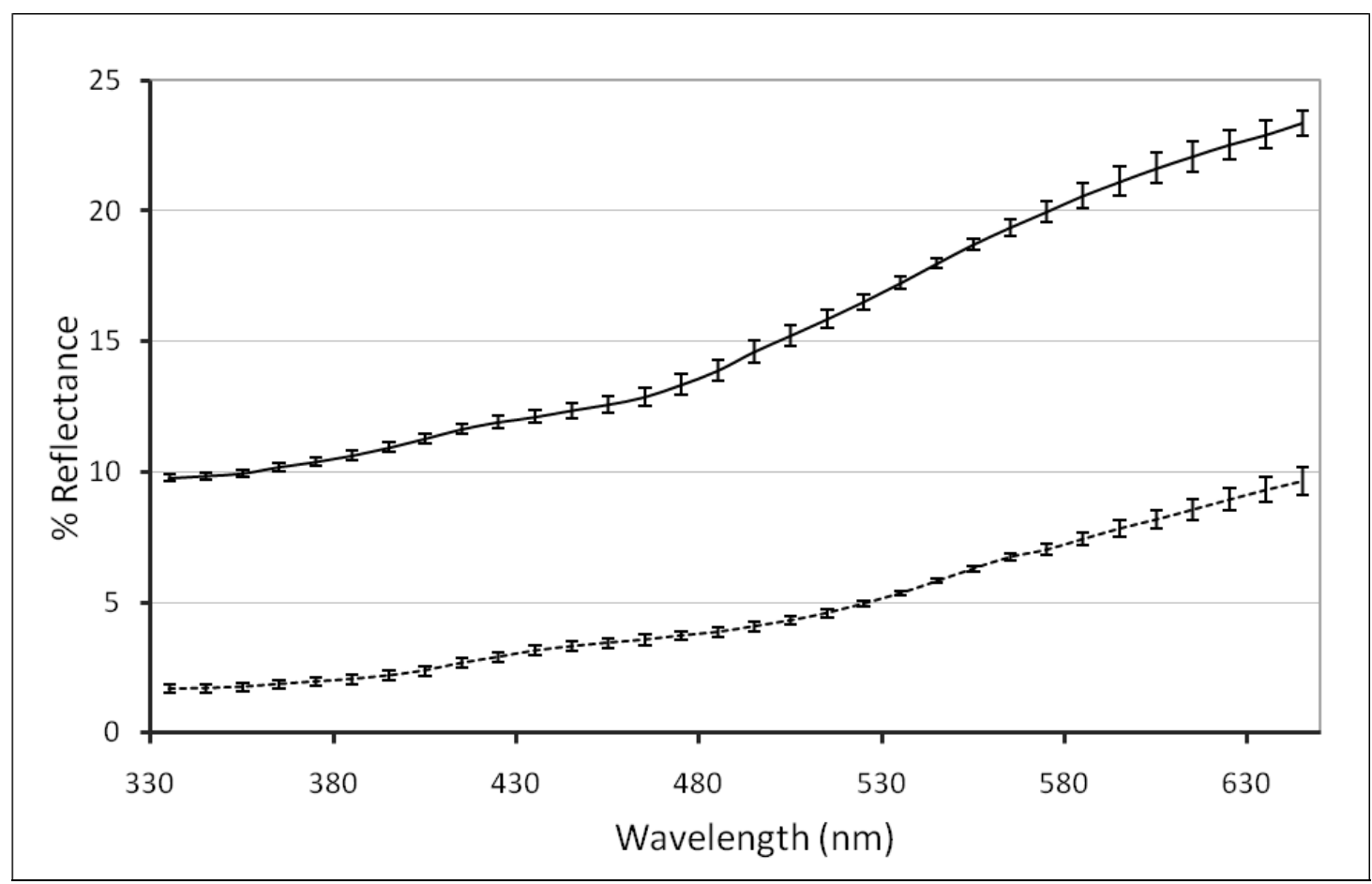

Figure 3.9. Mt. Hood population reflectance: bronze morph abdomen. Solid line $=$ male, dashed line $=$ female. Equalized for brightness, error bars are standard error of hue. Male $n=9$, female $n=6$.

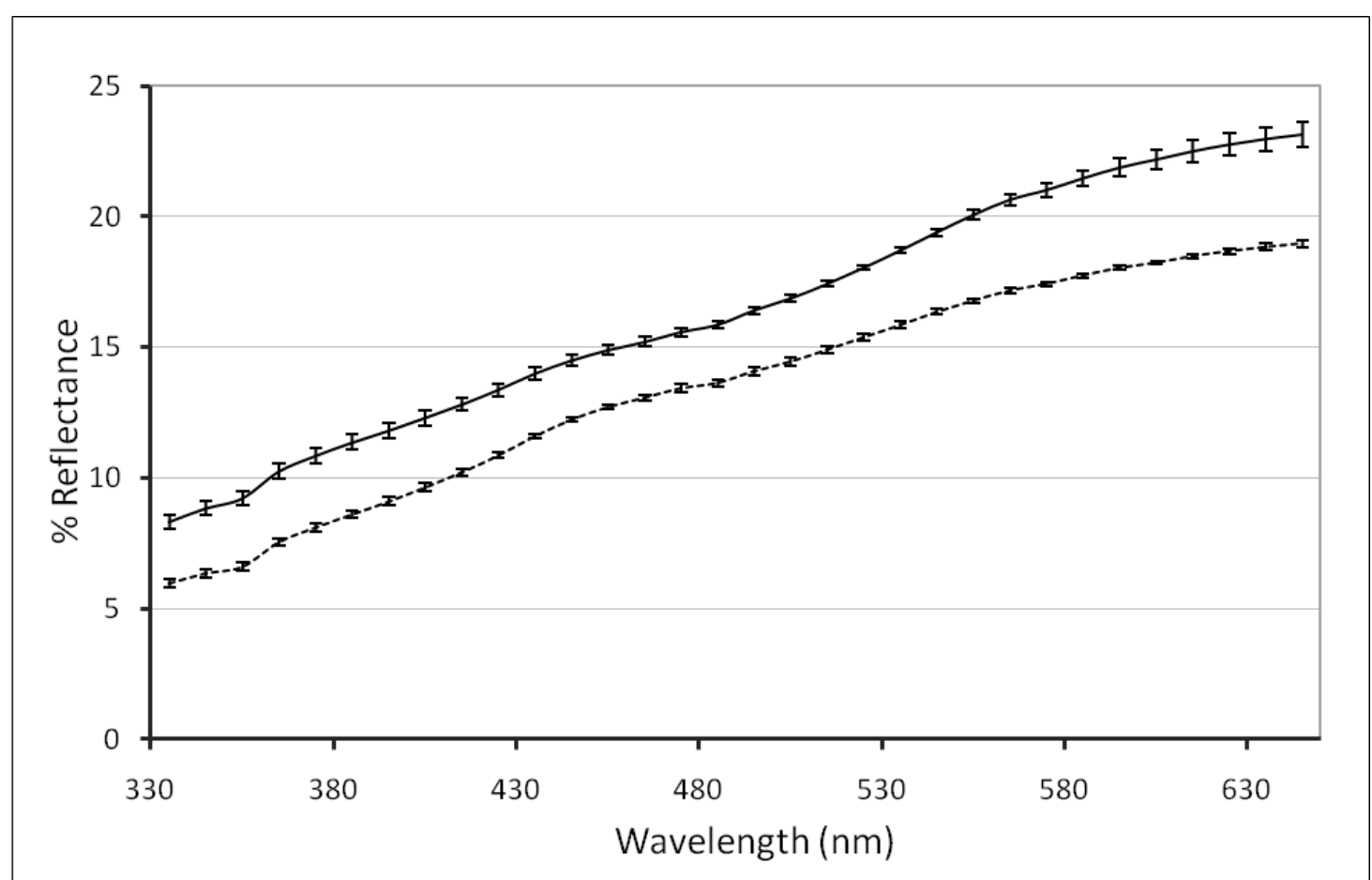

Figure 3.10. Gorge population reflectance: beige morph ventrum. Solid line $=$ male, dashed line $=$ female. Equalized for brightness, error bars are standard error of hue. Male $n=14$, female $n=7$. 


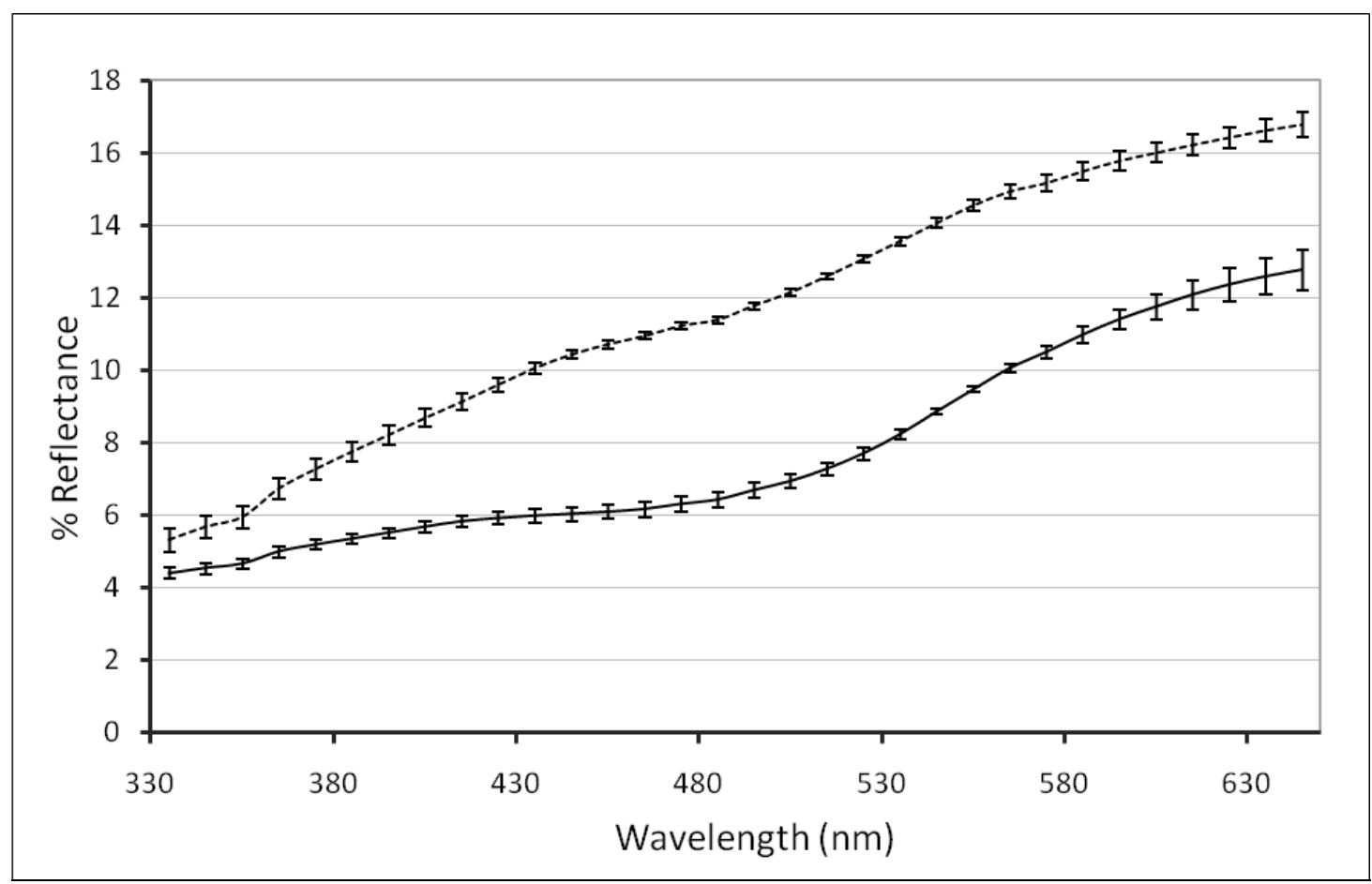

Figure 3.11. Mt. Hood population reflectance: bronze morph ventrum. Solid line $=$ male, dashed line $=$ female. Equalized for brightness, error bars are standard error of hue. Male $n=10$, female, $n=7$.

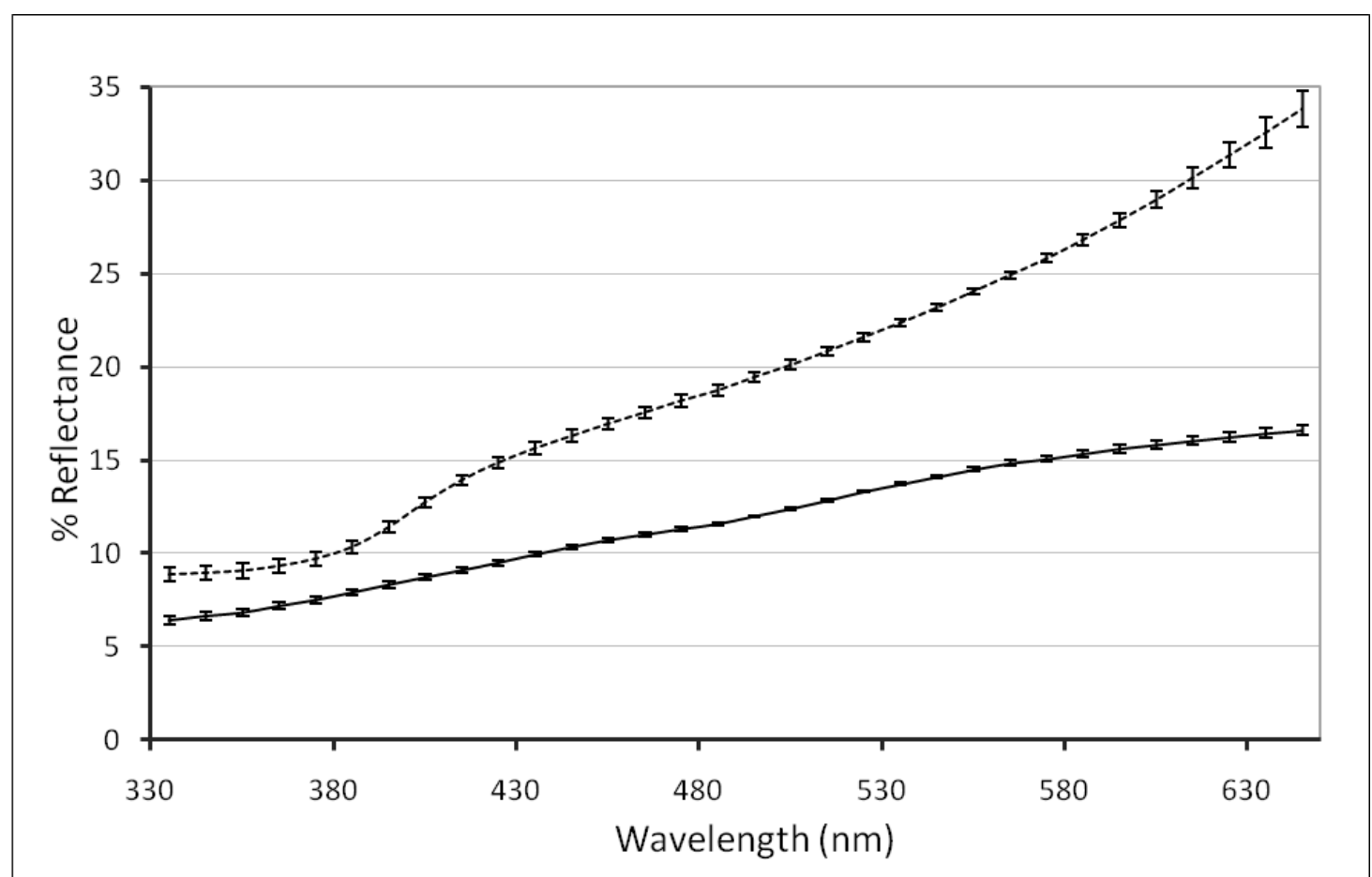

Figure 3.12. Gorge population habitat reflectance. Solid line = male and female habitat (rock), dashed line $=$ male and female habitat (leaves). Equalized for brightness, error bars are standard error of hue. Rock $n=16$, leaves $n=15$. 


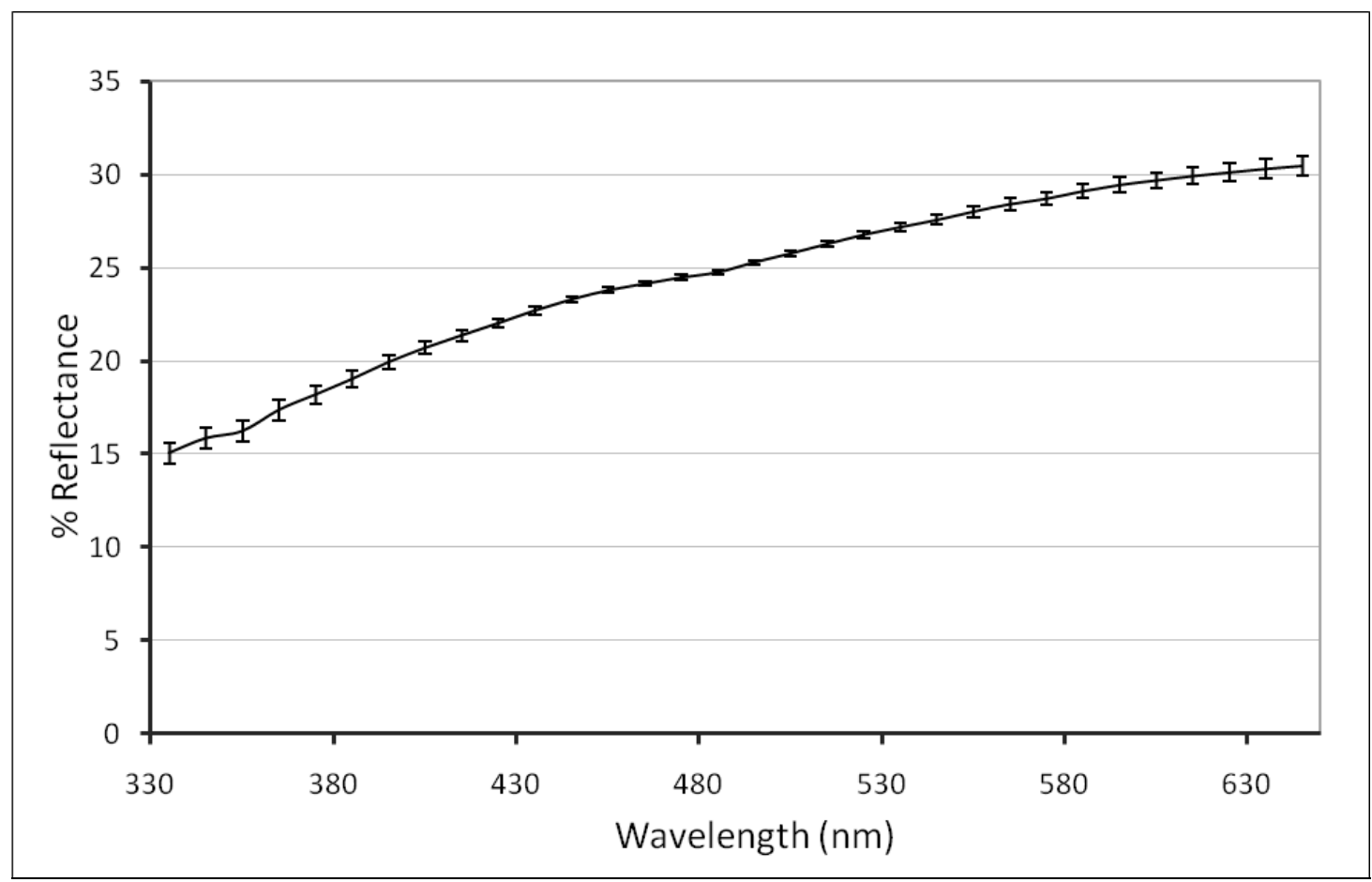

Figure 3.13. Mt. Hood population habitat reflectance. Male and female habitat (rock). Equalized for brightness, error bars are standard error of hue. $n=16$.

\section{Color Space Polar Plots}

For all color space plots I have replaced the quadrant labels 1-4 with the approximate hue names as interpreted by humans (decimals are due to the discreet values generated in the analog to digital conversion process of the spectrometer):

- Q1: 330.1-410.0nm, "ultraviolet"

- Q2: 410.2-489.8nm, “violet/blue"

- Q3: 490.0-569.9nm, "green"

- Q4: 570.1-649.9nm, "yellow/orange/red" 
I have also replaced like groups of spectra from discrete populations with polygon outlines tracing out the extent of their coverage within the color space.

Rocks were by far the most common habitat substrate upon which $H$. oregonensis were encountered, $92.2 \%$ of adults were associated with rock. Dry leaf litter, the main other substrate encountered in substantial numbers, was a distant second, which was associated with $36.4 \%$ of adults (these two habitat types were not necessarily mutually exclusive within a quadrat). Spiders that were encountered on or near leaf litter were mostly from the Gorge or Siskiyou populations ( 84.0 and 40.0\%, respectively); spiders from the Mt. Hood and Tillamook populations were seldom encountered on anything but bare rock $(0.0$ and $5.6 \%$ were associated with leaf litter, respectively.

In all plots I have included habitat reflectance spectra from two populations; because spiders of the Gorge and Siskiyou populations and the Mt. Hood and Tillamook populations have such similar coloration, a comparison of all substrate colors known to be found with each potential morph is warranted. Because leaf litter associations were so few at the Mt. Hood and Tillamook populations, rather than trace a polygon I have indicated their presence with an " $\mathrm{x}$ " (see legends).

Figures 3.14 through 3.17 show male and female beige morph spectra and their habitats. Figures 3.18 through 3.21 show male and female bronze morph spider reflectance spectra and their habitats. All figures incorporate solar irradiance data; descriptions can be found below, categorized by habitat and population. 


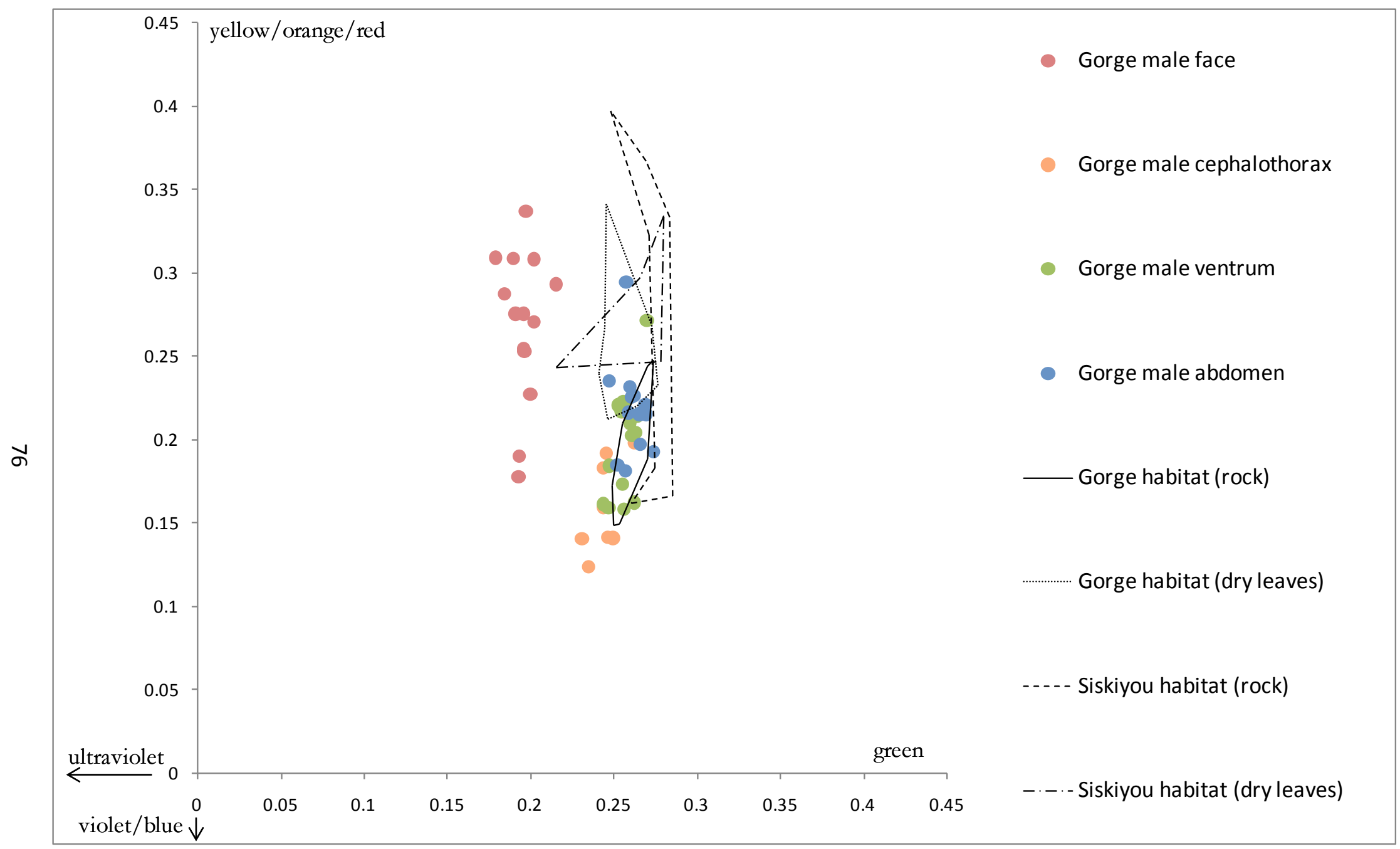

Figure 3.14. Gorge population, male hue and chroma color space polar plot: reflectance data combined with solar irradiance data. 


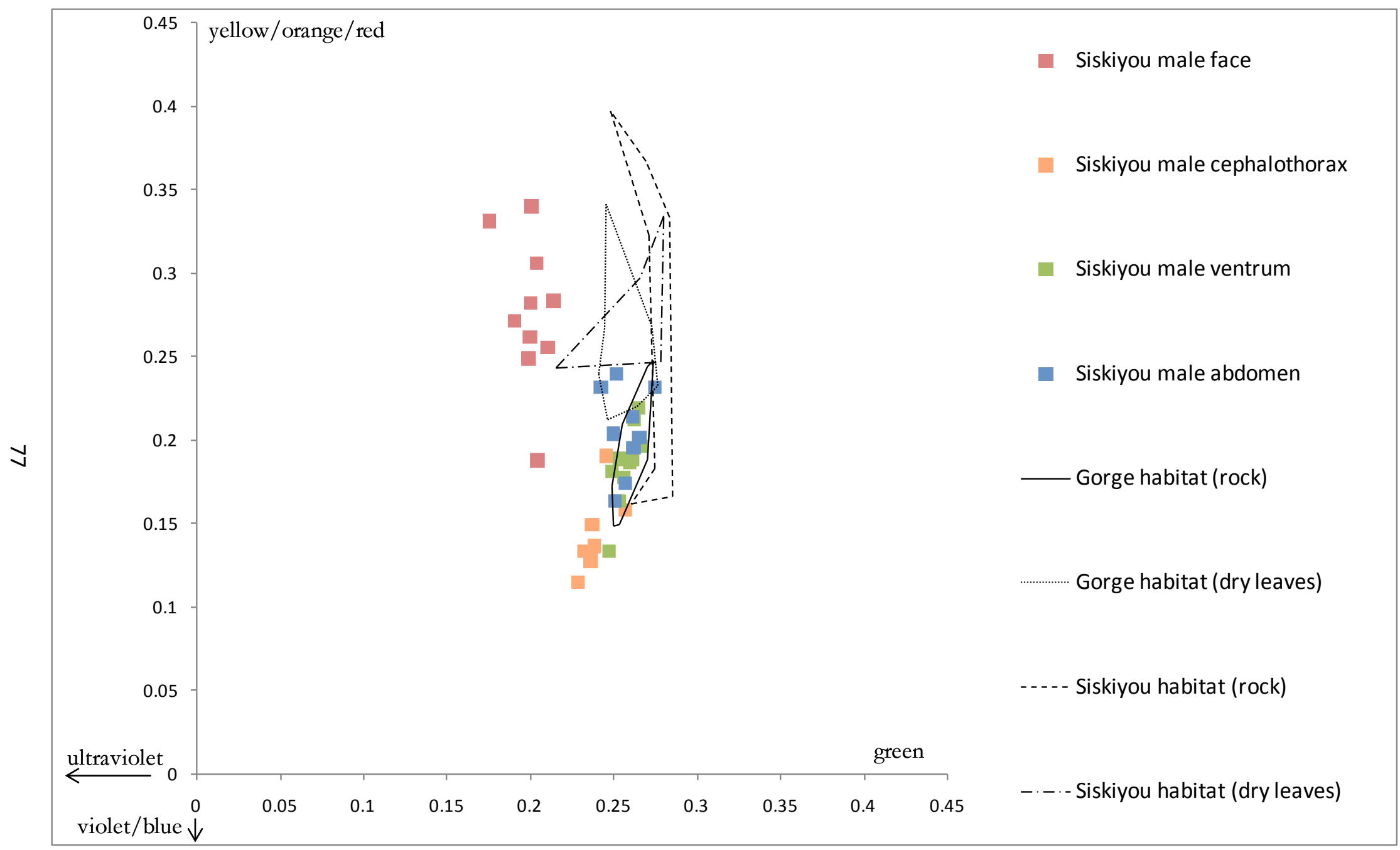

Figure 3.15. Siskiyou population, male hue and chroma color space polar plot: reflectance data combined with solar irradiance data. 


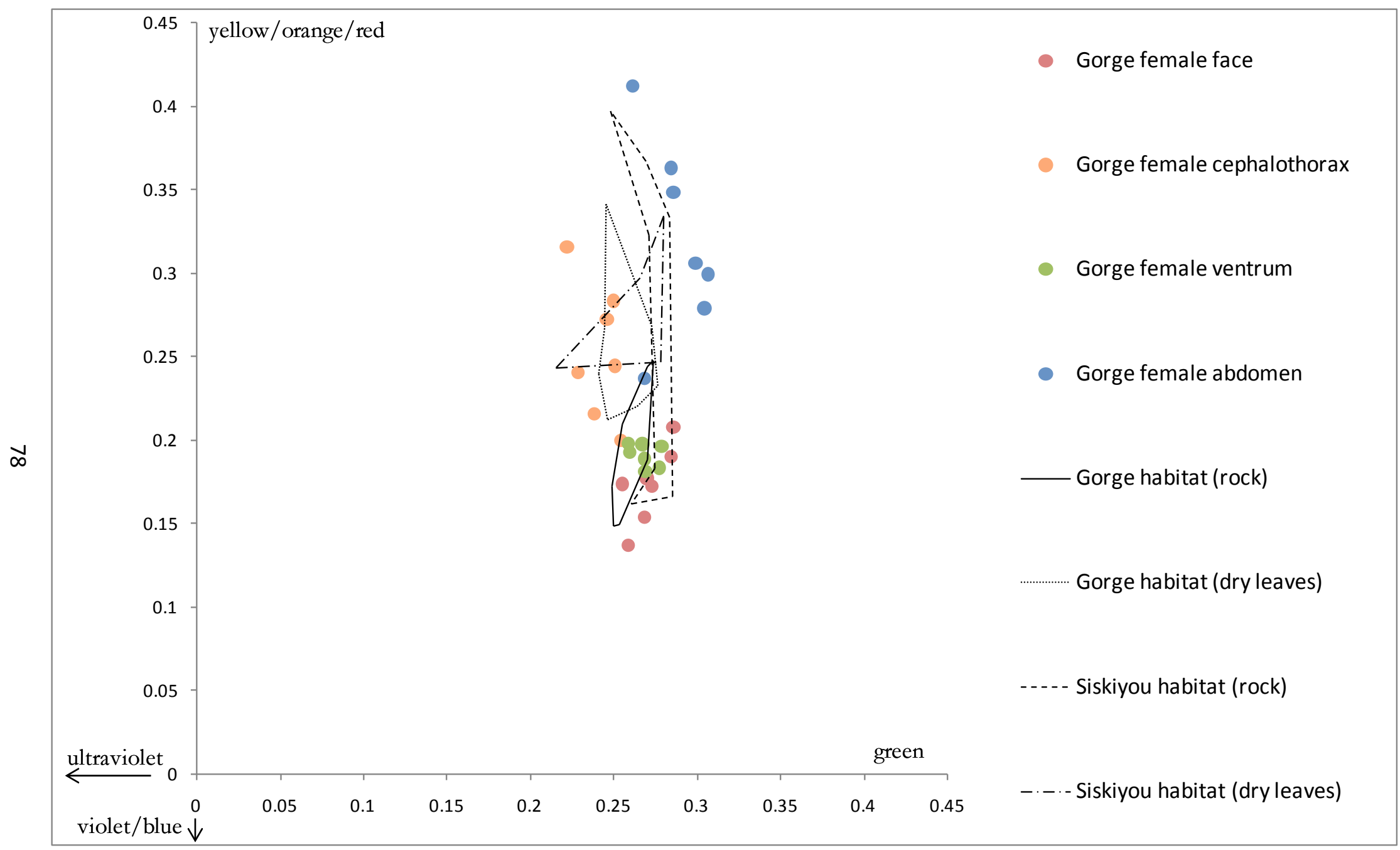

Figure 3.16. Gorge population, female hue and chroma color space polar plot: reflectance data combined with solar irradiance data. 


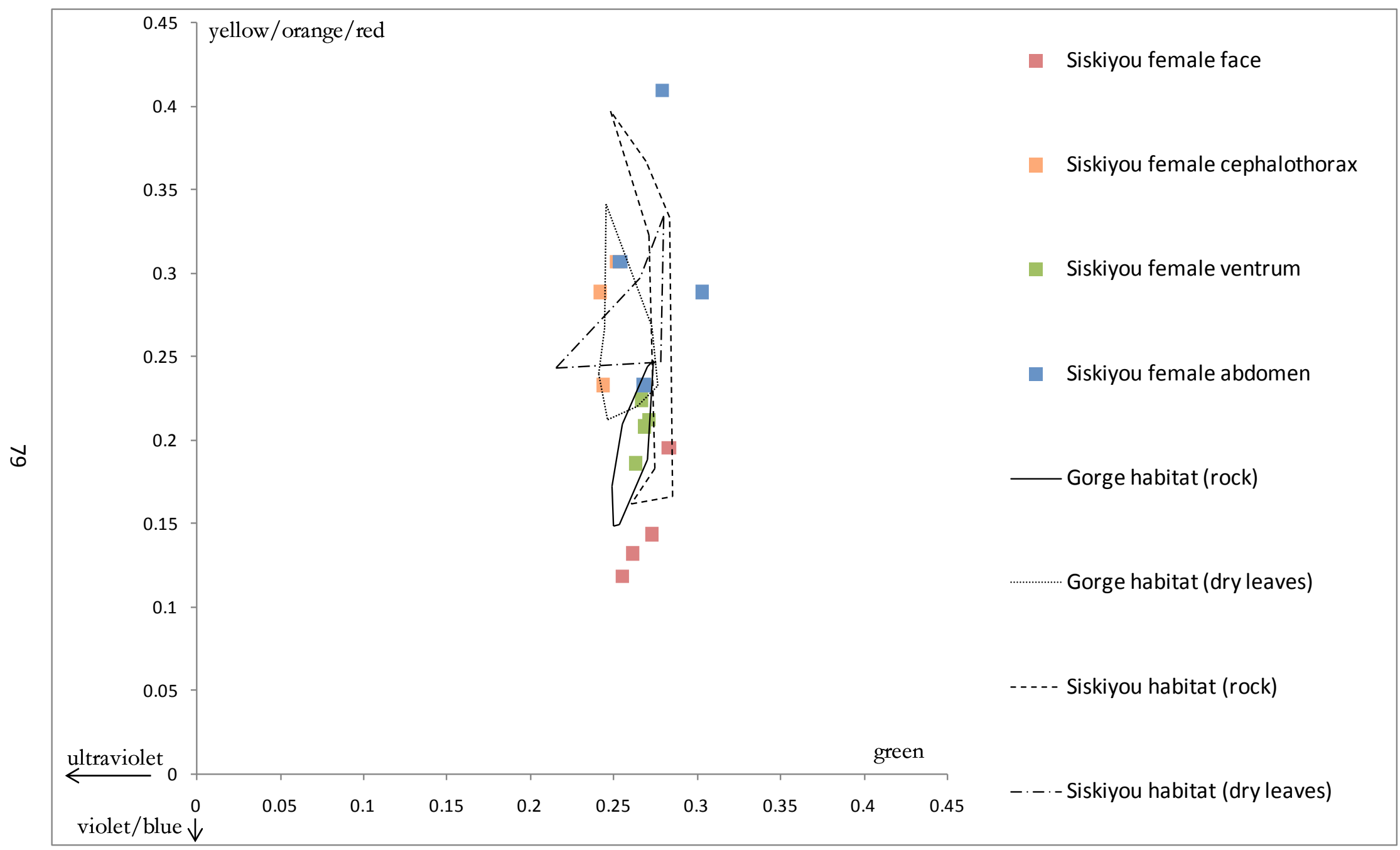

Figure 3.17. Siskiyou population, female hue and chroma color space polar plot: reflectance data combined with solar irradiance data. 


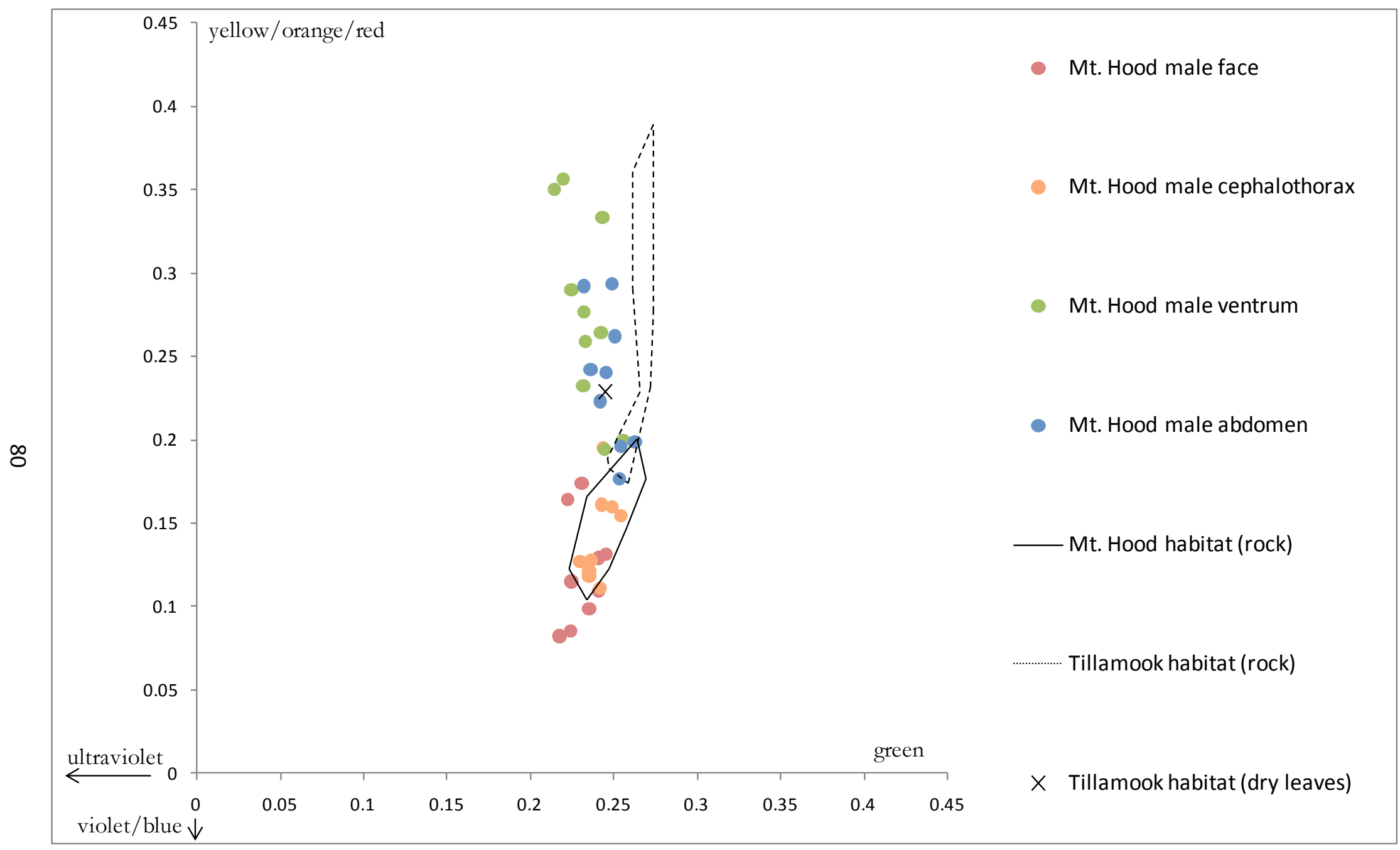

Figure 3.18. Mt. Hood population, male hue and chroma color space polar plot: reflectance data combined with solar irradiance data. 


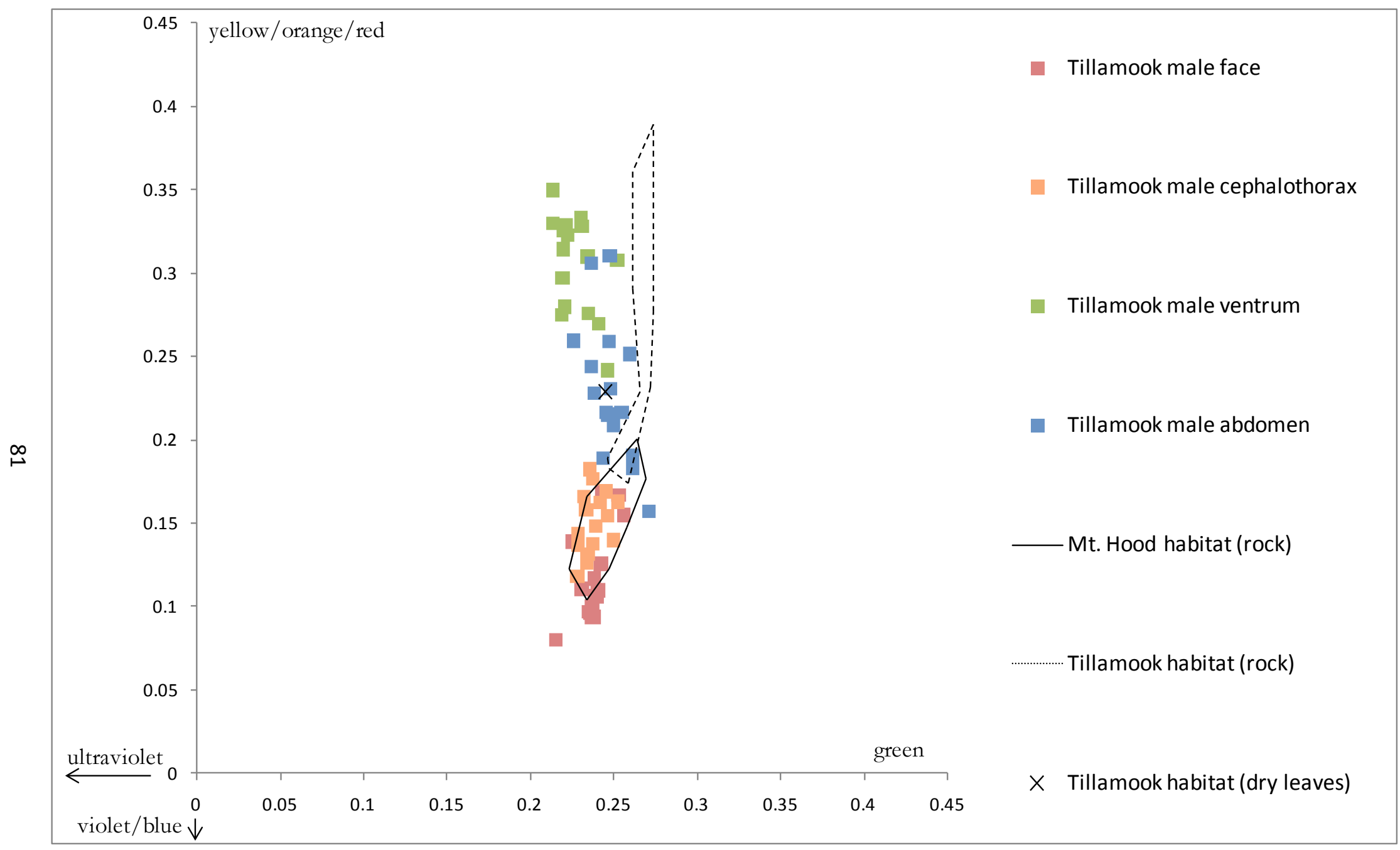

Figure 3.19. Tillamook population, male hue and chroma color space polar plot: reflectance data combined with solar irradiance data. 


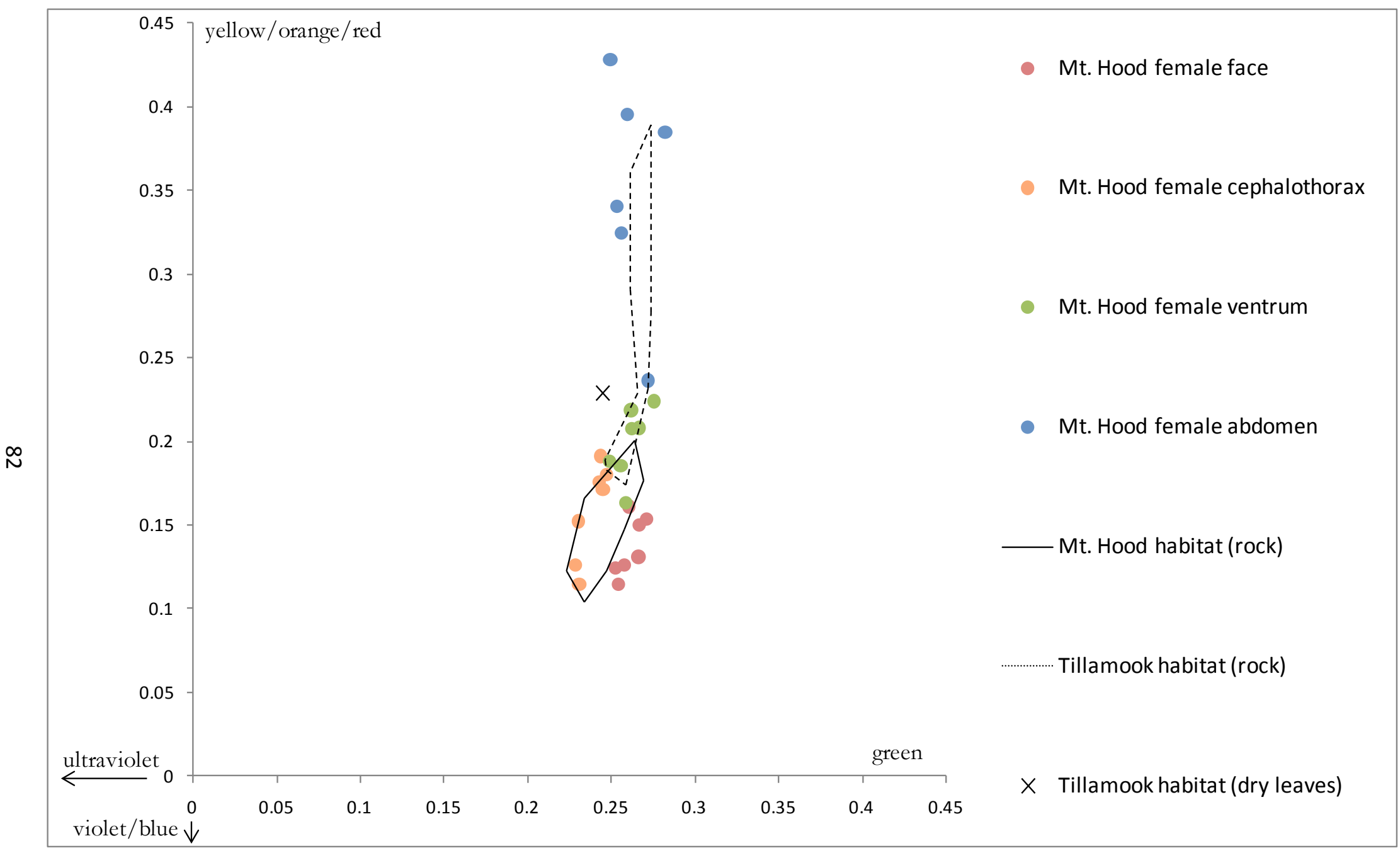

Figure 3.20. Mt. Hood population, female hue and chroma color space polar plot: reflectance data combined with solar irradiance data. 


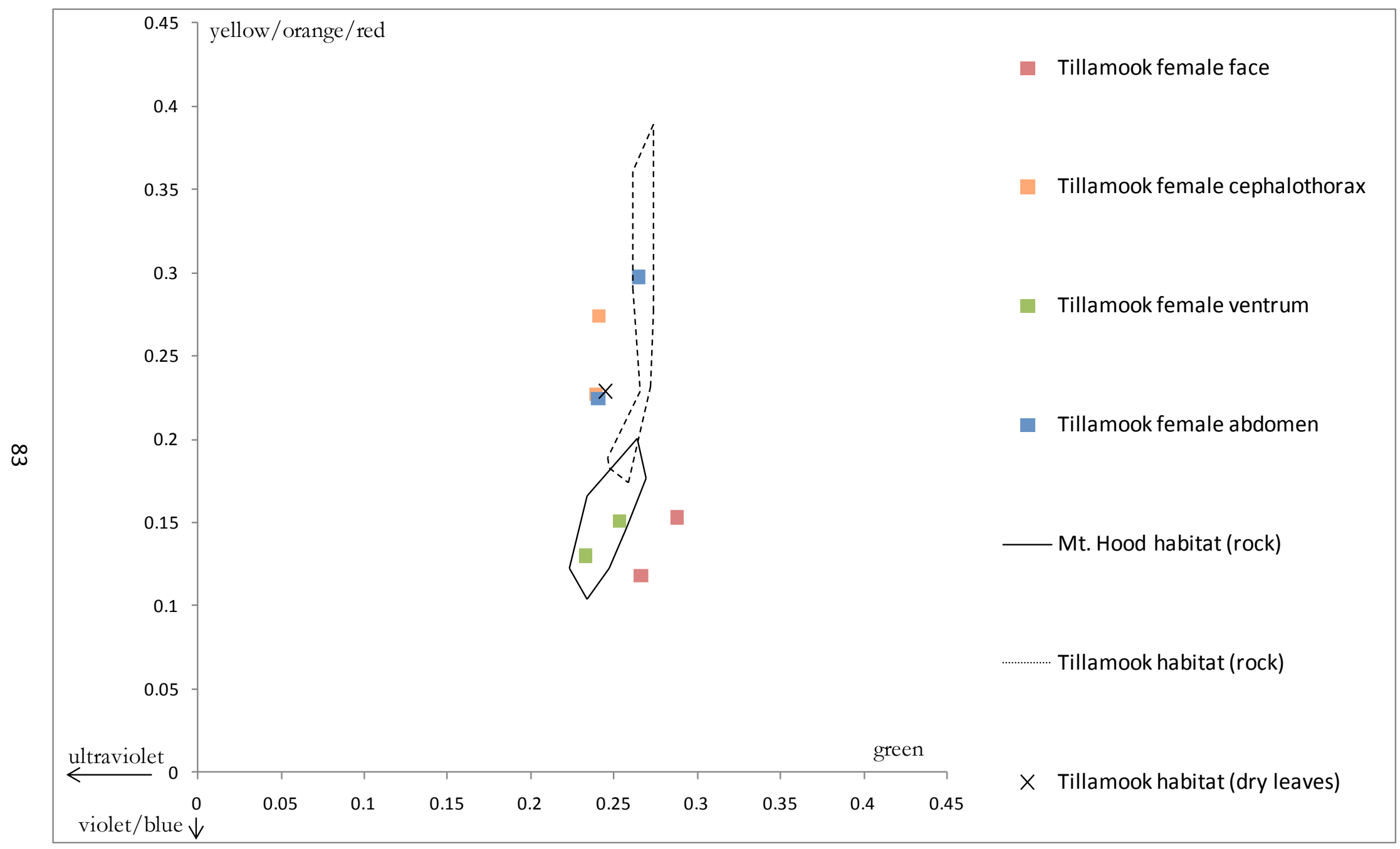

Figure 3.21. Tillamook population, female hue and chroma color space polar plot: reflectance data combined with solar irradiance data. 
Color Space Polar Plots: Habitat Data

The polygons enclosing the rock reflectance spectra of the collection sites of the Gorge and Siskiyou populations (the beige morph spiders, figures 3.14 through 3.17) are adjacent, but have little overlap. Combined, the rocks from the two sites form a roughly contiguous gradient that ranges from low chroma to moderate chroma. As the chroma increases, the hue shifts towards the longer wavelengths (yellow, orange, and red). Rocks from the Siskiyou habitat have a higher chroma and reflect more long-wavelength light than rocks from the Gorge habitat. There is a similar trend with the rocks from the Mt. Hood and Tillamook population habitats (the bronze morph spiders, figures 3.18 through 3.21). Not only are the rock reflectance spectra polygons from the two sites contiguous and nearly nonoverlapping, but the rocks from one site (Tillamook) both have a higher chroma and reflect more long-wavelength light than rocks from the other site (Mt. Hood). When the rock reflectance spectra of both of the beige morph sites are grouped together and compared to the collective bronze morph rock reflectance spectra, the overall difference in hue are small. Differences in chroma are more substantial: some of the Mt. Hood reflectance spectra have a markedly lower chroma than any of the other three sites.

Color Space Polar Plots: Gorge and Siskiyou Populations 
The greatest difference in combined hue and chroma between spider and habitat was the anterior region of the male Gorge and Siskiyou population spiders (Figures 3.14 and 3.15). The anterior reflectance spectra formed a distinct cluster separate from the other body regions of the same spiders, reflecting a greater amount of short (UV) and long (yellow/orange/red) wavelength light and less in the mid-range wavelengths (violet/blue and green). Differences in chroma between Gorge and Siskiyou population male anteriors and other body regions of the same spiders were negligible.

Contrary to Gorge and Siskiyou population male anteriors, however, female anteriors of these populations were very low in chroma and were not shifted towards the extreme ends of the spectrum (Figures 3.16 and 3.17). Female abdomens tended to have slightly higher chroma than their habitat, but overall no body region of the females deviated far from their associated habitat components. However, the abdomen reflectance spectra of females were distinctly more diffuse in their scatter than those of the male abdomen reflectance spectra. Much of this scatter can be attributed to variation in chroma; female hue tended to be rather well-centered on habitat hue.

Color Space Polar Plots: Mt. Hood and Tillamook Populations Unlike the anteriors of the male Gorge and Siskiyou population spiders, those of the Mt. Hood and Tillamook population males had low chroma (indeed, they had 
some of the lowest chroma of all reflectance spectra) and their hue matched the habitat reflectance spectra well (Figures 3.18 and 3.19). Collectively, the reflectance spectra of all the body regions of the male Mt. Hood and Tillamook population spiders are tightly clustered; within this cluster, the uppermost extreme in chroma was occupied by the abdomen and ventrum reflectance spectra. Like the anterior of the male Gorge and Siskiyou population spiders, however, the abdomen and ventrum of the Mt. Hood and Tillamook male spiders deviated from the habitat reflectance spectra by reflecting more in the ultraviolet range and less in the green range. This difference is not as great as that of the Gorge and Siskiyou population male anteriors.

Female Mt. Hood and Tillamook spiders generally match the habitat better than that of their male counterparts, but as with the Gorge and Siskiyou population spiders, some the female body region reflectance spectra (especially the abdomen) are less tightly grouped than those of the males (Figures 3.20 and 3.21). The one body region of females that is a noticeable mismatch with the habitat (while rather minor) is the anterior. Female anterior reflectance spectra are slightly offset from the habitat reflectance spectra as well as male anterior reflectance spectra; female anteriors have a little bit higher chroma than male anteriors and are less reflective of UV and more reflective of green than the male anteriors and the habitat.

Differences in Spider and Habitat Hue, Chroma, and Brightness 
I compared the differences in raw hue, chroma, and brightness values of spiders and their habitat with Student's $t$-tests. Only those comparisons that pertained to the findings outlined in the color space polar plots were evaluated; for complete pairwise comparisons of raw hue, chroma, and brightness values between spiders and habitat see tables A-1 through A-3 in the appendix. As was apparent in the color space polar plots, the hues of Gorge and Siskiyou population male anterior reflectance spectra were significantly different from all Gorge and Siskiyou population habitat types (Table 3.1). Interestingly, the hues of Gorge and Siskiyou population female anterior reflectance spectra were significantly different from all Gorge and Siskiyou population habitat types, as well. Gorge and Siskiyou male and female anterior chroma were both significantly different from Siskiyou rocks, but not significantly different from Gorge rocks (Table 3.2). Gorge and Siskiyou male anterior brightness were significantly different from all Gorge and Siskiyou habitat types, while Gorge and Siskiyou female anteriors were only significantly different from Siskiyou rocks and Gorge dry leaves (Table 3.3).

Table 3.1. Student's t-test comparing raw hue values of Gorge and Siskiyou habitat reflectance spectra with all Gorge and Siskiyou male and female body regions. Reflectance data combined with solar irradiance data. Listed below are P-values, $\alpha=0.05$.

\begin{tabular}{lccccc}
\hline \hline & $n$ & Gorge rock & Siskiyou rock & Gorge dryleaf & Siskiyou dryleaf \\
\hline$n$ & & 16 & 11 & 15 & 4 \\
Gorge female abdomen & 7 & 0.002 & 0.458 & 0.140 & 0.841 \\
Gorge female cephalothorax & 7 & 0.002 & 0.757 & 0.329 & 0.712 \\
Gorge female face & 7 & 0.009 & 0.001 & 0.000 & 0.001 \\
Gorge female ventrum & 7 & 0.278 & 0.007 & 0.000 & 0.006
\end{tabular}




\begin{tabular}{llllll} 
Gorge male abdomen & 4 & 0.032 & 0.103 & 0.008 & 0.018 \\
Gorge male cephalothorax & 3 & 0.122 & 0.003 & 0.000 & 0.001 \\
Gorge male face & 4 & 0.000 & 0.016 & 0.000 & 0.029 \\
Gorge male ventrum & 4 & 0.634 & 0.025 & 0.000 & 0.005 \\
Siskiyou female abdomen & 6 & 0.040 & 0.554 & 0.330 & 0.888 \\
Siskiyou female cephalothorax & 7 & 0.038 & 0.909 & 0.738 & 0.395 \\
Siskiyou female face & 7 & 0.023 & 0.001 & 0.002 & 0.001 \\
Siskiyou female ventrum & 7 & 0.398 & 0.033 & 0.002 & 0.009 \\
Siskiyou male abdomen & 2 & 0.211 & 0.057 & 0.004 & 0.009 \\
Siskiyou male cephalothorax & 2 & 0.004 & 0.001 & 0.000 & 0.000 \\
Siskiyou male face & 2 & 0.000 & 0.017 & 0.000 & 0.029 \\
Siskiyou male ventrum & 2 & 0.418 & 0.008 & 0.000 & 0.003 \\
\hline
\end{tabular}

Table 3.2. Student's t-test comparing raw chroma values of Gorge and Siskiyou habitat reflectance spectra with all Gorge and Siskiyou male and female body regions. Reflectance data combined with solar irradiance data. Listed below are P-values, $\alpha=0.05$.

\begin{tabular}{lccccc}
\hline \hline & $n$ & Gorge rock & Siskiyou rock & Gorge dryleaf & Siskiyou dryleaf \\
\hline$n$ & & 16 & 11 & 15 & 4 \\
Gorge female abdomen & 7 & 0.000 & 0.133 & 0.004 & 0.131 \\
Gorge female cephalothorax & 7 & 0.047 & 0.035 & 0.285 & 0.278 \\
Gorge female face & 7 & 0.732 & 0.001 & 0.001 & 0.074 \\
Gorge female ventrum & 7 & 0.469 & 0.003 & 0.000 & 0.107 \\
Gorge male abdomen & 4 & 0.046 & 0.008 & 0.007 & 0.172 \\
Gorge male cephalothorax & 3 & 0.013 & 0.000 & 0.000 & 0.029 \\
Gorge male face & 4 & 0.450 & 0.005 & 0.013 & 0.123 \\
Gorge male ventrum & 4 & 0.932 & 0.001 & 0.000 & 0.083 \\
Siskiyou female abdomen & 6 & 0.050 & 0.568 & 0.170 & 0.402 \\
Siskiyou female cephalothorax & 7 & 0.187 & 0.032 & 0.250 & 0.229 \\
Siskiyou female face & 7 & 0.275 & 0.002 & 0.016 & 0.039 \\
Siskiyou female ventrum & 7 & 0.122 & 0.007 & 0.011 & 0.154 \\
Siskiyou male abdomen & 2 & 0.543 & 0.003 & 0.001 & 0.107 \\
Siskiyou male cephalothorax & 2 & 0.000 & 0.000 & 0.000 & 0.017 \\
Siskiyou male face & 2 & 0.159 & 0.015 & 0.093 & 0.188 \\
Siskiyou male ventrum & 2 & 0.336 & 0.001 & 0.000 & 0.064 \\
\hline
\end{tabular}

Table 3.3. Student's $t$-test comparing raw brightness values of Gorge and Siskiyou habitat reflectance spectra with all Gorge and Siskiyou male and female body regions. Reflectance data combined with solar irradiance data. Listed below are P-values, $\alpha=0.05$.

$n$ Gorge rock Siskiyou rock Gorge dryleaf Siskiyou dryleaf 


\begin{tabular}{lccccc}
$n$ & & 16 & 11 & 15 & 4 \\
Gorge female abdomen & 7 & 0.000 & 0.000 & 0.000 & 0.035 \\
Gorge female cephalothorax & 7 & 0.271 & 0.001 & 0.000 & 0.130 \\
Gorge female face & 7 & 0.792 & 0.000 & 0.000 & 0.081 \\
Gorge female ventrum & 7 & 0.129 & 0.001 & 0.000 & 0.144 \\
Gorge male abdomen & 4 & 0.417 & 0.001 & 0.000 & 0.115 \\
Gorge male cephalothorax & 3 & 0.000 & 0.014 & 0.002 & 0.301 \\
Gorge male face & 4 & 0.000 & 0.000 & 0.000 & 0.032 \\
Gorge male ventrum & 4 & 0.001 & 0.015 & 0.003 & 0.302 \\
Siskiyou female abdomen & 6 & 0.000 & 0.000 & 0.000 & 0.030 \\
Siskiyou female cephalothorax & 7 & 0.668 & 0.004 & 0.018 & 0.111 \\
Siskiyou female face & 7 & 0.912 & 0.004 & 0.017 & 0.096 \\
Siskiyou female ventrum & 7 & 0.269 & 0.008 & 0.024 & 0.166 \\
Siskiyou male abdomen & 2 & 0.809 & 0.000 & 0.000 & 0.088 \\
Siskiyou male cephalothorax & 2 & 0.013 & 0.006 & 0.001 & 0.225 \\
Siskiyou male face & 2 & 0.000 & 0.000 & 0.000 & 0.032 \\
Siskiyou male ventrum & 2 & 0.093 & 0.001 & 0.000 & 0.151 \\
\hline
\end{tabular}

Student's t-test comparisons of Mt. Hood population male anteriors and their habitat revealed no significant difference in hue (though there was a significant difference between male anteriors and Tillamook rocks), as suggested in the color space polar plots (Table 3.4). However, hue of Tillamook population male anteriors was significantly different from both Mt. Hood and Tillamook rock hue. Hue of both Mt. Hood and Tillamook female anteriors were not significantly different from Mt. Hood rocks, though they were significantly different from Tillamook rocks. Mt. Hood and Tillamook male anterior chroma were both significantly different from both Mt. Hood and Tillamook rock chroma (Table 3.5). Chroma of neither Mt. Hood nor Tillamook female anteriors was significantly different from Mt. Hood rock chroma, and while chroma of Tillamook female anteriors was not significantly different from 
chroma of Tillamook rocks, Mt. Hood female anteriors were significantly different.

Brightness of Mt. Hood and Tillamook male and female anteriors were all significantly

different from brightness of both Mt. Hood and Tillamook rocks (Table 3.6).

Table 3.4. Student's $t$-test comparing raw hue values of Mt. Hood and Tillamook habitat reflectance spectra with all Gorge and Siskiyou male and female body regions. Note that there are no Mt. Hood population leaf reflectance spectra to compare, nor are there enough Tillamook population leaf reflectance spectra to compare. Reflectance data combined with solar irradiance data. Listed below are P-values, $\alpha=0.05$.

\begin{tabular}{lccc}
\hline \hline & $n$ & Mt. Hood rock & Tillamook rock \\
\hline$n$ & & 16 & 16 \\
Mt. Hood female abdomen & 13 & 0.000 & 0.031 \\
Mt. Hood female cephalothorax & 9 & 0.102 & 0.000 \\
Mt. Hood female face & 14 & 0.075 & 0.000 \\
Mt. Hood female ventrum & 14 & 0.000 & 0.000 \\
Mt. Hood male abdomen & 9 & 0.000 & 0.543 \\
Mt. Hood male cephalothorax & 7 & 0.949 & 0.000 \\
Mt. Hood male face & 10 & 0.221 & 0.000 \\
Mt. Hood male ventrum & 10 & 0.000 & 0.154 \\
Tillamook female abdomen & 9 & 0.081 & 0.818 \\
Tillamook female cephalothorax & 9 & 0.077 & 0.722 \\
Tillamook female face & 9 & 0.250 & 0.009 \\
Tillamook female ventrum & 10 & 0.903 & 0.000 \\
Tillamook male abdomen & 16 & 0.000 & 0.255 \\
Tillamook male cephalothorax & 16 & 0.066 & 0.000 \\
Tillamook male face & 16 & 0.006 & 0.000 \\
Tillamook male ventrum & 16 & 0.000 & 0.000 \\
\hline
\end{tabular}

Table 3.5. Student's t-test comparing raw chroma values of Mt. Hood and Tillamook habitat reflectance spectra with all Gorge and Siskiyou male and female body regions. Note that there are no Mt. Hood population leaf reflectance spectra to compare, nor are there enough Tillamook population leaf reflectance spectra to compare. Reflectance data combined with solar irradiance data. Listed below are P-values, $\alpha=0.05$.

\begin{tabular}{lccc}
\hline \hline & $n$ & Mt. Hood rock & Tillamook rock \\
\hline$n$ & & 16 & 16 \\
Mt. Hood female abdomen & 13 & 0.000 & 0.032 \\
Mt. Hood female cephalothorax & 9 & 0.839 & 0.000 \\
& 90 & &
\end{tabular}




\begin{tabular}{lccl} 
Mt. Hood female face & 14 & 0.180 & 0.000 \\
Mt. Hood female ventrum & 14 & 0.000 & 0.002 \\
Mt. Hood male abdomen & 9 & 0.000 & 0.026 \\
Mt. Hood male cephalothorax & 7 & 0.572 & 0.000 \\
Mt. Hood male face & 10 & 0.014 & 0.000 \\
Mt. Hood male ventrum & 10 & 0.000 & 0.394 \\
Tillamook female abdomen & 9 & 0.257 & 0.732 \\
Tillamook female cephalothorax & 9 & 0.145 & 0.264 \\
Tillamook female face & 9 & 0.391 & 0.070 \\
Tillamook female ventrum & 10 & 0.826 & 0.013 \\
Tillamook male abdomen & 16 & 0.000 & 0.009 \\
Tillamook male cephalothorax & 16 & 0.642 & 0.000 \\
Tillamook male face & 16 & 0.013 & 0.000 \\
Tillamook male ventrum & 16 & 0.000 & 0.865 \\
\hline
\end{tabular}

Table 3.6. Student's t-test comparing raw brightness values of Mt. Hood and Tillamook habitat reflectance spectra with all Gorge and Siskiyou male and female body regions. Note that there are no Mt. Hood population leaf reflectance spectra to compare, nor are there enough Tillamook population leaf reflectance spectra to compare. Reflectance data combined with solar irradiance data. Listed below are P-values, $\alpha=0.05$.

\begin{tabular}{lccc}
\hline \hline & $n$ & Mt. Hood rock & Tillamook rock \\
\hline$n$ & & 16 & 16 \\
Mt. Hood female abdomen & 13 & 0.000 & 0.000 \\
Mt. Hood female cephalothorax & 9 & 0.000 & 0.076 \\
Mt. Hood female face & 14 & 0.000 & 0.000 \\
Mt. Hood female ventrum & 14 & 0.000 & 0.042 \\
Mt. Hood male abdomen & 9 & 0.000 & 0.556 \\
Mt. Hood male cephalothorax & 7 & 0.000 & 0.022 \\
Mt. Hood male face & 10 & 0.000 & 0.000 \\
Mt. Hood male ventrum & 10 & 0.000 & 0.000 \\
Tillamook female abdomen & 9 & 0.000 & 0.006 \\
Tillamook female cephalothorax & 9 & 0.000 & 0.017 \\
Tillamook female face & 9 & 0.000 & 0.000 \\
Tillamook female ventrum & 10 & 0.002 & 0.065 \\
Tillamook male abdomen & 16 & 0.000 & 0.560 \\
Tillamook male cephalothorax & 16 & 0.000 & 0.001 \\
Tillamook male face & 16 & 0.000 & 0.000 \\
Tillamook male ventrum & 16 & 0.000 & 0.000 \\
\hline
\end{tabular}


The $t$-test comparison data also lend some support to the hypothesis that there may be subtle differences in hue and chroma between Mt. Hood and Tillamook population female and male faces, first suggested in the color space plots. The differences in hue and brightness are nonsignificant (Tables 3.7 and 3.9), though the difference in chroma is significant for Mt. Hood population (though not Tillamook population) (Table 3.8).

Table 3.7. Student's $t$-test pairwise comparison of raw hue values of Mt. Hood and Tillamook male and female face reflectance spectra. Reflectance data combined with solar irradiance data. Listed below are P-values, $\alpha=0.05$.

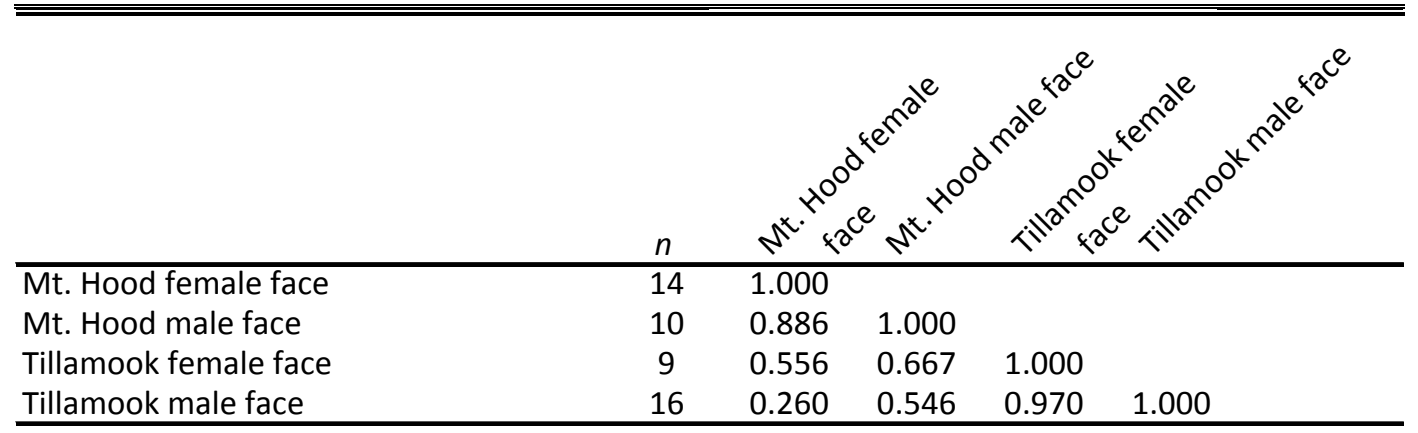

Table 3.8. Student's $t$-test pairwise comparison of raw chroma values of Mt. Hood and Tillamook male and female face reflectance spectra. Reflectance data combined with solar irradiance data. Listed below are P-values, $\alpha=0.05$.

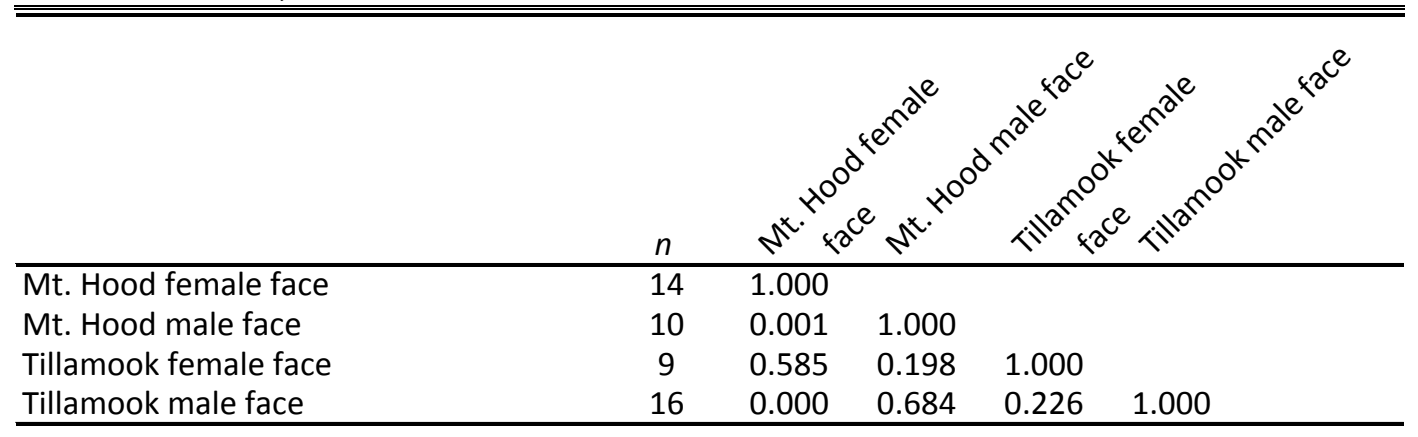


Table 3.9. Student's $t$-test pairwise comparison of raw brightness values of Mt. Hood and Tillamook male and female face reflectance spectra. Reflectance data combined with solar irradiance data. Listed below are P-values, $\alpha=0.05$.

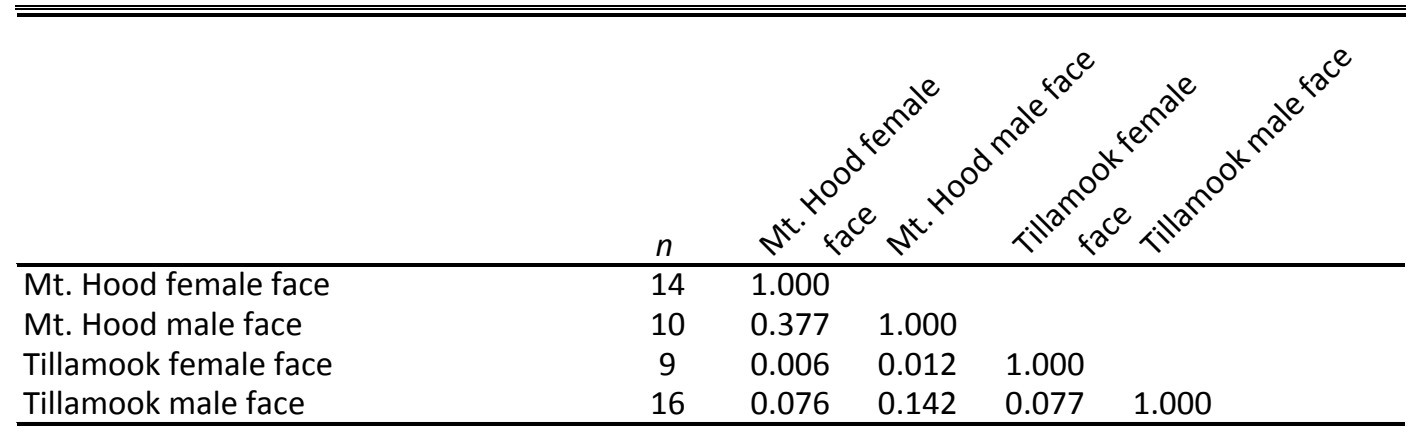

Standard deviations of abdomen raw chroma values were substantially higher

for females of all populations than for males (Table 3.10). This suggests that female abdomens have more variation in chroma while male abdomens are more tightly clustered in color space. Standard deviations in raw hue values were also higher for female abdomens, though these trends were not as dramatic. Differences in standard deviations for brightness did not form any consistent trends. 
Table 3.10. Standard Deviation of raw color values of all spider reflectance spectra sorted by population, sex, and body region as well as habitat reflectance spectra grouped by population. Note that there are no Mt. Hood population leaf reflectance spectra to compare, nor are there enough Tillamook population leaf reflectance spectra to compare. Reflectance data combined with solar irradiance data.

\begin{tabular}{|c|c|c|c|c|}
\hline & $n$ & Brightness & Hue & Chroma \\
\hline Gorge female abdomen & 7 & 3401.866 & 0.102 & 0.041 \\
\hline Gorge female cephalothorax & 7 & 6701.014 & 0.092 & 0.026 \\
\hline Gorge female face & 7 & 8957.144 & 0.049 & 0.021 \\
\hline Gorge female ventrum & 7 & 5085.479 & 0.025 & 0.006 \\
\hline Siskiyou female abdomen & 4 & 4003.081 & 0.116 & 0.059 \\
\hline Siskiyou female cephalothorax & 3 & 7931.345 & 0.060 & 0.020 \\
\hline Siskiyou female face & 4 & 11541.542 & 0.074 & 0.027 \\
\hline Siskiyou female ventrum & 4 & 9784.617 & 0.034 & 0.012 \\
\hline Mt. Hood female abdomen & 6 & 3926.345 & 0.112 & 0.051 \\
\hline Mt. Hood female cephalothorax & 7 & 10012.785 & 0.074 & 0.022 \\
\hline Mt. Hood female face & 7 & 5119.719 & 0.046 & 0.013 \\
\hline Mt. Hood female ventrum & 7 & 8400.750 & 0.044 & 0.018 \\
\hline Tillamook female abdomen & 2 & 3337.137 & 0.065 & 0.049 \\
\hline Tillamook female cephalothorax & 2 & 3535.962 & 0.064 & 0.025 \\
\hline Tillamook female face & 2 & 1512.259 & 0.050 & 0.025 \\
\hline Tillamook female ventrum & 2 & 4289.260 & 0.019 & 0.020 \\
\hline Gorge male abdomen & 13 & 6153.563 & 0.065 & 0.019 \\
\hline Gorge male cephalothorax & 9 & 5450.466 & 0.078 & 0.024 \\
\hline Gorge male face & 14 & 3157.799 & 0.091 & 0.036 \\
\hline Gorge male ventrum & 14 & 8899.916 & 0.071 & 0.024 \\
\hline Siskiyou male abdomen & 9 & 4009.823 & 0.064 & 0.018 \\
\hline Siskiyou male cephalothorax & 7 & 6863.208 & 0.062 & 0.019 \\
\hline Siskiyou male face & 10 & 3457.241 & 0.092 & 0.033 \\
\hline Siskiyou male ventrum & 10 & 6237.554 & 0.055 & 0.018 \\
\hline Mt. Hood male abdomen & 9 & 9668.724 & 0.101 & 0.025 \\
\hline Mt. Hood male cephalothorax & 9 & 5915.197 & 0.075 & 0.019 \\
\hline Mt. Hood male face & 9 & 3576.620 & 0.105 & 0.019 \\
\hline Mt. Hood male ventrum & 10 & 6533.540 & 0.126 & 0.038 \\
\hline Tillamook male abdomen & 16 & 10321.968 & 0.104 & 0.025 \\
\hline Tillamook male cephalothorax & 16 & 8027.148 & 0.053 & 0.014 \\
\hline Tillamook male face & 16 & 2452.468 & 0.080 & 0.019 \\
\hline Tillamook male ventrum & 16 & 6260.403 & 0.064 & 0.021 \\
\hline Gorge rock & 16 & 11397.854 & 0.061 & 0.022 \\
\hline Mt. Hood rock & 16 & 10354.170 & 0.059 & 0.022 \\
\hline Tillamook rock & 16 & 7152.506 & 0.111 & 0.050 \\
\hline Siskiyou rock & 11 & 19590.341 & 0.164 & 0.055 \\
\hline Gorge dryleaf & 15 & 9717.489 & 0.066 & 0.022 \\
\hline Siskiyou dryleaf & 4 & 23596.290 & 0.065 & 0.047 \\
\hline
\end{tabular}


Differences in Hue, Chroma, and Brightness Contrast Values between Spiders and Habitat

Table 3.11 explores the differences in the amount of contrast of body regions versus habitat substrate compared between males and females. There is a significant difference in the amount of brightness and hue+chroma contrast between male Gorge population anteriors and rock substrate versus female Gorge population anteriors and rock substrate; the same holds true for Siskiyou spider anteriors compared to Siskiyou rocks (Table 3.11). These results support the observation that there is a difference in degree of crypsis between male and female beige morph anteriors (first suggested by the distinct grouping of male beige morph anterior reflectance spectra in the color space polar plots). There are conflicting results regarding the differences in habitat contrast between Mt. Hood and Tillamook population male and female anteriors; for the Mt. Hood population, there is a significant difference in hue+chroma but not brightness, and for the Tillamook population neither hue+chroma nor brightness are significantly different from each other (Table 3.11). 
Table 3.11. Contrast values of females of a single population versus the average of all (male and female) habitat reflectance spectra from their own population compared against the contrast values of males of the same population versus the average of all (male and female) habitat reflectance spectra from their own population. Number of habitat reflectance measurements: Gorge rock $=16$, Mt. Hood rock $=16$, Siskiyou rock $=11$, Tillamook rock $=16$, Gorge leaf $=15$, Siskiyou leaf $=4$. Not enough individuals from the Mt. Hood and Tillamook populations were encountered on leaf litter to run a comparison for that habitat type. Reflectance data combined with solar irradiance data. Listed below are $P$-values, $\alpha=0.05$.

\begin{tabular}{|c|c|c|c|c|c|c|}
\hline & \multirow[b]{2}{*}{$n$ (males) } & \multirow[b]{2}{*}{$n$ (females) } & \multicolumn{2}{|c|}{ ROCKS } & \multicolumn{2}{|c|}{ LEAVES } \\
\hline & & & Brightness & Hue + Chroma & Brightness & Hue + Chroma \\
\hline Gorge population abdomen & 13 & 7 & 0.000 & 0.007 & 0.000 & 0.443 \\
\hline Gorge population face & 14 & 7 & 0.002 & 0.000 & 0.002 & 0.576 \\
\hline Gorge population ventrum & 14 & 7 & 0.016 & 0.007 & 0.015 & 0.234 \\
\hline Siskiyou population abdomen & 9 & 4 & 0.003 & 0.545 & 0.003 & 0.197 \\
\hline Siskiyou population face & 10 & 4 & 0.046 & 0.042 & 0.045 & 0.010 \\
\hline Siskiyou population ventrum & 10 & 4 & 0.912 & 0.111 & 0.914 & 0.120 \\
\hline Mt. Hood population abdomen & 9 & 6 & 0.000 & 0.012 & & \\
\hline Mt. Hood population cephalothorax & 9 & 7 & 0.006 & 0.254 & & \\
\hline Mt. Hood population face & 9 & 7 & 0.388 & 0.037 & & \\
\hline Mt. Hood population ventrum & 10 & 7 & 0.005 & 0.001 & & \\
\hline Tillamook population abdomen & 16 & 2 & 0.032 & 0.020 & & \\
\hline Tillamook population face & 16 & 2 & 0.097 & 0.791 & & \\
\hline Tillamook population ventrum & 16 & 2 & 0.355 & 0.015 & & \\
\hline
\end{tabular}


Summary

The main results regarding hue and chroma of the spiders are summarized in Tables 3.12 through 3.15 . 
Table 3.12. Summary of main results, Gorge population.

\begin{tabular}{|c|c|c|c|}
\hline & Face & Cephalothorax & Abdomen \\
\hline Male & $\begin{array}{l}\text { More short (UV), and long (red) } \\
\text { wavelength reflectance, less mid-range } \\
\text { (blue, green) wavelength reflectance } \\
\text { compared to other body regions. } \\
\text { - Hue does not match habitat } \\
\text { (substantial disparity). }\end{array}$ & $\begin{array}{l}\text { - Hue matches habitat, less chroma than } \\
\text { habitat. }\end{array}$ & - Hue and chroma match habitat. \\
\hline Female & $\begin{array}{l}\text { - } \quad \text { Low chroma. } \\
\text { - Hue and chroma match habitat. }\end{array}$ & $\begin{array}{l}\text { - Hue and chroma generally match } \\
\text { habitat, especially leaves. }\end{array}$ & $\begin{array}{l}\text { - } \quad \text { Generally matches hue of habitat. } \\
\text { Moderately high chroma compared to } \\
\text { habitat; substantial scatter outside of } \\
\text { habitat range. }\end{array}$ \\
\hline
\end{tabular}

$œ$ Table 3.13. Summary of main results, Siskiyou population.

\begin{tabular}{|c|c|c|c|}
\hline & Face & Cephalothorax & Abdomen \\
\hline Male & 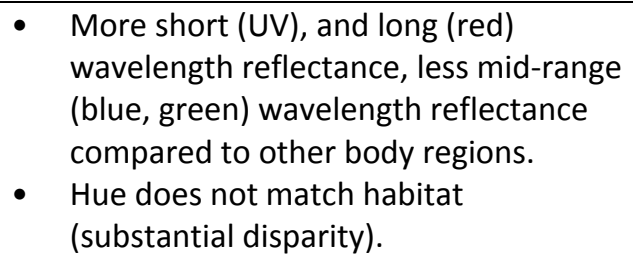 & $\begin{array}{l}\text { - Hue matches habitat, less chroma than } \\
\text { habitat. }\end{array}$ & - Hue and chroma match habitat. \\
\hline Female & $\begin{array}{l}\text { - } \quad \text { Low chroma. } \\
\text { - Hue and chroma match habitat. }\end{array}$ & $\begin{array}{l}\text { - Hue and chroma generally match } \\
\text { habitat, especially leaves. }\end{array}$ & $\begin{array}{l}\text { - Generally matches hue of habitat. } \\
\text { - } \text { hoderately high chroma compared to } \\
\text { habitat; substantial scatter outside of } \\
\text { habitat range. }\end{array}$ \\
\hline
\end{tabular}


Table 3.14. Summary of main results, Mt. Hood population.

\begin{tabular}{|c|c|c|c|}
\hline & Face & Cephalothorax & Abdomen \\
\hline Male & $\begin{array}{l}\text { - } \quad \text { Low chroma. } \\
\text { - Hue and chroma match habitat. }\end{array}$ & $\begin{array}{l}\text { - } \quad \text { Low chroma. } \\
\text { - Hue and chroma match habitat. }\end{array}$ & $\begin{array}{l}\text { - Hue moderately deviates from habitat. } \\
\text { More short (UV) and less mid-range } \\
\text { (blue, green) wavelength reflectance } \\
\text { compared to habitat. } \\
\text { - Chroma matches habitat. }\end{array}$ \\
\hline Female & $\begin{array}{l}\text { - Low chroma. } \\
\text { - Less short (UV) and more mid-range } \\
\text { (blue, green) wavelength reflectance } \\
\text { compared with habitat. }\end{array}$ & $\begin{array}{l}\text { - } \quad \text { Reflectance spectra are scattered } \\
\text { widely among individuals. } \\
\text { - } \quad \text { Low chroma. } \\
\text { - Hue and chroma match habitat. }\end{array}$ & $\begin{array}{l}\text { - } \quad \text { Reflectance spectra are scattered } \\
\text { widely among individuals. } \\
\text { - Moderately high chroma. } \\
\text { - Hue and chroma match habitat. }\end{array}$ \\
\hline
\end{tabular}

$\bullet$ Table 3.15. Summary of main results, Tillamook population. Note that there are only two females available from this population, so trends should be interpreted with caution.

\begin{tabular}{|c|c|c|c|}
\hline & Face & Cephalothorax & Abdomen \\
\hline Male & $\begin{array}{l}\text { - } \quad \text { Low chroma. } \\
\text { - } \quad \text { Hue and chroma match habitat. }\end{array}$ & $\begin{array}{l}\text { - } \quad \text { Moderate chroma. } \\
\text { - } \quad \text { Hue and chroma match habitat. }\end{array}$ & $\begin{array}{l}\text { - Hue moderately deviates from habitat. } \\
\text { More short (UV) and less mid-range } \\
\text { (blue, green) wavelength reflectance } \\
\text { compared to habitat. } \\
\text { - Chroma matches habitat. }\end{array}$ \\
\hline Female & $\begin{array}{l}\text { - Low chroma. } \\
\text { - Less short (UV) and more mid-range } \\
\text { (blue, green) wavelength reflectance } \\
\text { compared with habitat. }\end{array}$ & $\begin{array}{l}\text { - } \text { Reflectance spectra are scattered } \\
\text { widely among individuals. } \\
\text { - } \quad \text { Low chroma. } \\
\text { - Hue and chroma match habitat. }\end{array}$ & $\begin{array}{l}\text { - } \quad \text { Reflectance spectra are scattered } \\
\text { widely among individuals. } \\
\text { - } \quad \text { Moderately high chroma. } \\
\text { - Hue and chroma match habitat. }\end{array}$ \\
\hline
\end{tabular}




\section{DISCUSSION}

The clearest way to make sense of the superabundance of data available in exploratory color studies such as this is to begin with the color space polar plots. These provide a lucid diagram of trends which can be further investigated by testing the raw color data for significance as well as the degree of contrast of the animals with their habitat. This method of analysis provides direction when sifting through the numerous possible comparisons, allowing one to first discover a trend and then check its significance rather than trying to uncover a trend in a raw table of significance values. An important caveat to keep in mind, however, is that significance values generated with a $t$-test are not necessarily rooted in biological significance. If the animal is particularly sensitive to a specific region of the spectrum, for example, subtle differences in that region (which may not register as statistically significant) may indeed be noticeable to the animal and therefore be biologically significant.

The high degree of brightness disparity between many spider body regions and their habitat as well as the difficulty experienced in extracting patterns from these data was somewhat surprising. However, an unusual bias in the vision systems of insects (and perhaps in other taxa as well) may explain this. Chromatic contrast (hue and chroma) play a far more important role in vision for insects than does achromatic contrast (brightness) (Backhaus, 1991; Chittka et al., 1992; Chittka et al., 1994; Fukushi, 1990; Green and Flint, 1986; Théry et al., 2005; Zschokke, 2002). This 
could mean that the insect predators and prey that interact with $\mathrm{H}$. oregonensis may be largely ignoring this component of vision, in which case there may be relaxed natural selection in the achromatic channel of the spiders' coloration. If spiders also focus on the chromatic channel and downplay the achromatic channel, there could also be sexual selection implications. For these reasons, I will focus on hue and chroma for my discussion of the results.

The fairly minimal differences in hue between the collective groupings of beige morph and bronze morph rocky substrates, and the fact that a population of each morph was encountered at a habitat at either end of the overall range in chroma implies that the spiders are somewhat generalist with regards to the chroma of their substrate - at least within the range of the habitat samples measured in this study.

Males of the Gorge and Siskiyou populations possess cryptic coloration on exposed body regions which is consistent with natural selection acting on this region of the body exposed to aerial predators. Dorsal hue and chroma of female cephalothorax and abdomen reflectance spectra, however, were more variable than expected when compared to the same body regions of males, especially on the abdomen. This results in the abdomen region of females possessing substantially reduced crypsis.

Gorge and Siskiyou populations male anteriors possess lower crypsis compared to female anteriors. Male anteriors were dramatically less cryptic that 
those of females when considering hue and chroma; this is consistent with sexual selection pressure acting on male anteriors for noncryptic coloration.

While cephalothorax reflectance spectra of males of the Mt. Hood and Tillamook populations did generally match the habitat well for hue and chroma, the abdomen reflectance spectra were found to have too much UV/too little green to be completely cryptic, despite relatively low variation among individuals. Surprisingly, female anteriors matched the habitat better than male anteriors. However, there was a substantial amount of variation among individuals, which sometimes compromised their crypsis (it's worth noting here that the sample size of Tillamook females was small, and therefore conclusions should be interpreted with caution).

Unlike the beige morph populations, the Mt. Hood and Tillamook populations did not have lower male anterior crypsis compared to female anteriors. While the relative amount of hue and chroma crypsis were similar between males and females (both possessing very low chroma), it was males that actually matched the habitat more closely, while females deviated from the habitat by having a slightly greater reflectance of green/lower reflectance of UV.

The variability of abdomen hue and chroma of females of all four populations and particularly the much-reduced crypsis of beige morph female abdomens was a surprising find. If they are subject to the same natural selection pressures of males without sexual selection pressures, they would be expected to have equal or greater levels of crypsis. A possible explanation for this apparent incongruity is that the 
assumption that the sexes are exposed to the same natural selective pressures is incorrect. The unexpectedly low dorsal crypsis of females as well as a comparatively high degree of variation among individuals may be caused by lower selection for crypsis among females than among males. This could be the case if females were to spend less time in exposed habitats than males, and were thus at less risk of predation. While not enough of the natural history of these spiders is known to confirm this possibility, it would agree with anecdotal evidence; it would seem that upon molting to adulthood, males are in the exposed courtship habitat until they die, whereas females most likely enter this high-risk habitat only briefly in order to mate, subsequently retreating to a more protected habitat to lay their eggs (see chapter 2 for more details on their natural history). Alternatively, females (and juveniles, which have nearly identical coloration) may simply be well-adapted to an entirely different habitat that was not measured in this study; they may actually spend most of their lives in - and be more cryptic upon - another habitat type altogether. It seems most plausible that females and juveniles spend most of their lives in areas not investigated in this study; the collection sites were most likely mating arenas. The presence of juveniles in these habitats (although comparatively few in number) implies that the primary habitat of these spiders is probably near these mating arenas, though captured juveniles could have been in the process of dispersing away from their site of hatching. 
The relatively tight clustering of dorsal reflectance spectra measurements of males of the Gorge and Siskiyou populations implies that hue and chroma selection is particularly strong, allowing for little variation among individuals. The matching of this clustering with the spiders' habitat hue and chroma supports the possibility that this selection is for crypsis. However, the reflectance spectra of abdomens of males of Mt. Hood and Tillamook populations differ from their habitat. The fact that juvenile bronze morph dorsal hue and chroma (which, while unconfirmed with spectrometer readings, appears to be nearly identical to female coloration) matches their collection habitat better than that of males implies that selective pressure is actively pushing male abdomens away from crypsis. It is unusual that this deviation from crypsis is on the dorsum: this is a body region that most likely plays a comparatively small role in courtship displays compared to the anterior region. The reduced crypsis of the male dorsal regions likely does increase the risk of predation (perhaps the reason why the deviation from crypsis is comparatively modest), but if the associated increase in mating success is great enough, the risk will be warranted.

The anterior hue and chroma of the Gorge and Siskiyou populations were both substantially different from their habitat and the relative degree of crypsis was much lower than that of their female counterparts. Considering the conspicuous role of male spiders' anterior in courtship displays, it is to be expected that this region would be highly scrutinized by females and perhaps subject to selection based on the females' preferences. While it is possible that differences in anterior coloration 
between males and females may simply be due to sex recognition cues, such a dramatic difference between the sexes - in which male colorations are much less cryptic than female colorations - supports the hypothesis that female choice has exerted substantial influence here. It is worth noting here that the colorimetric direction in which beige morph male anteriors have diverged from crypsis is the same as that of the bronze morph male abdomens - both regions are relatively more reflective of UV and less reflective of green. This may be indicative of a generalized female sensory bias, conserved in both morphs, selecting for male reflectance with a higher UV to green ratio.

The hue and chroma of the anterior regions of the Mt. Hood and Tillamook population males and females were quite similar - there is no difference in hue and chroma apparent to the human eye - though the spectra were in fact separated into discrete (albeit immediately adjacent) clusters in the color space polar plots. Surprisingly, the anteriors of males were slightly more cryptic than those of females (visible on the color space polar plots, though not statistically significant). It is evident from these results that female choice has had little, if any impact on the degree of crypsis of bronze morph male anteriors. The fact that they are slightly different from female anteriors may be a sexual recognition cue. Indeed, the hue and chroma of bronze morph female anteriors are extremely close to those of beige morph females (Figures 3.18 through 3.21), and differ from bronze morph male 
anteriors in a colorimetric direction opposite to that of all male deviations from crypsis measured: they are less reflective of UV and more reflective of green.

The test of significance between differences in hue and chroma contrast between Tillamook population male and female anteriors and their habitat is nonsignificant because the means of the reflectance spectra of male and female anteriors are roughly equidistant from the mean of the habitat reflectance spectra not necessarily because the male and female anteriors are identical. Although the female sample size is too low to make any solid conclusions, this does suggest that there may be a very low or nonexistent level of biological significance in differences in crypsis between male and female anteriors. While the comparisons of raw hue and chroma values between Tillamook population males and females also returned nonsignificant differences, this may be due to a particularly small female sample size. The significant difference in raw chroma values between male and female Mt. Hood anteriors (and the similarity in coloration between Mt. Hood and Tillamook population anteriors) supports the possibility of a subtle but potentially biologically significant difference between bronze morph male and female anteriors. 
CHAPTER 4: Geographic Variation in Sexual Dimorphism and Male Sexual Ornaments Among Different Populations of Habronattus oregonensis

\section{CHAPTER OVERVIEW}

If natural or sexual selection is acting upon more than one character of an organism simultaneously and to a substantial degree, it is essential to consider these multiple factors in unison. Failure to do so may lead one to underestimate the intensity of selective pressure or overlook a selection regime on a character or suite of characters with substantial relevance to the overall evolution of the organism.

Here I attempt to quantify morphological variation of a prominent sexual ornament among different populations of the jumping spider Habronattus oregonensis. These results should be considered alongside those of chapter 3 , where I investigated the differences in sexual dichromatism, interpopulation differences in color, and differences in the degree of crypsis between sexes and among populations. The combination of these two datasets represent a more inclusive picture of the selective forces shaping male phenotype, exploring the possibility that different populations may have different intensities of selection on different male sexual characters.

Individuals of the Mt. Hood and Tillamook populations (both of the bronze color morph) were found to be larger than individuals of the Gorge and Siskiyou populations (both of the beige color morph) while none of the populations of the same color morphs were significantly different from each other in overall size. Male 
tibia of the Mt. Hood and Tillamook populations were found to be proportionally larger than the tibia of the males of the Gorge and Siskiyou populations, even when body size was factored out. Again, there was no significant difference in tibia size between populations of the same color morphs. This may indicate more intensive sexual selection for larger tibia among males of the Mt. Hood and Tillamook populations compared to the males of the Gorge and Siskiyou populations. Additionally, Siskiyou population males were found to have a slightly higher ratio of tibial fringe area to underlying integument compared to Gorge population males. This trend may indicate that males of the Siskiyou population rely more heavily on setae to increase apparent size of their tibia than Gorge males, perhaps a strategy to reduce resource investment in these sexual ornaments.

Based on the results of this study, it seems likely that female choice is shaping not only male coloration, but male first tibia morphology. The degree to which one or the other character is modified may directly depend on how much the other character has been modified.

\section{INTRODUCTION}

Geographic variation in sexual dimorphism has been documented in a number of different systems (Kwiatkowski and Sullivan, 2002; Macedonia et al., 2004; McCoy et al., 1994; McCoy et al., 1997; Wikelski and Trillmich, 1997). The mechanisms that generate this interpopulational variation can be quite diverse. For 
example, Wikelski and Trillmich (1997) demonstrated that constant pressure for larger body size due to intrasexual competition for mates can interact with local fluctuations in resource availability, resulting in dramatically different body size ranges for different populations of the same species. Kwiatkowski and Sullivan (2002) noted an example of geographic variation among populations in which sexual selection likely drove male coloration, though environmental conditions affected the degree to which sexual selection was evident: a more dispersed population likely increased female search costs for mates to the point where sexual selection for male coloration was absent. Variation in populations and reduced gene flow can ultimately lead to reproductive isolation and speciation; examples at the earlier stages of such divergences provide insight into the creation of species diversity (Arnqvist et al., 2000; Lande, 1981; Seehausen and van Alphen, 1998; Seehausen et al., 1997).

Previous work that I conducted demonstrated that male $H$. oregonensis possess greatly enlarged tibia on the first pair of legs, the lateral edges of which are lined with a dense fringe of setae. This character is found in both the beige and the bronze color morphs, and is an immediately recognizable diagnostic character for this species. The absence of such ornaments in females, the conspicuous anterior placement of these ornaments, and the prominent position these ornaments occupy in the males' courtship display strongly suggests that these are a sexually selected character. If tibia size is subject to sexual selection and there are differences in 
sexual selection pressures among populations - as suggested by my analysis of their reflectance properties - tibia size may vary. I therefore hypothesized that there would be significant differences in morphology among populations of $H$. oregonensis. Additionally, I investigated whether any such differences among populations correlated with the two color morphs encountered in this study, and if populations of the same morph possessed similar morphology.

\section{MATERIALS AND METHODS}

Populations Sampled

The sample size for males is larger and more uniform across the four populations in comparison to that of the females (Table 4.1). Therefore, comparisons among individual populations can only be made among males; there are not enough females to make valid comparisons at this level of distinction. Comparisons among females can, however, be made when populations from the same color morph are grouped together. While this reduces the ability to detect variation among females, it does allow for broader trends to surface. See chapter 2 for detailed descriptions of the collection sites.

Table 4.1. Sample sizes for morphometric analyses. Gorge and Siskiyou populations are beige morphs, Mt. Hood and Tillamook populations are bronze morphs.

\begin{tabular}{lcccc}
\hline \hline & \multicolumn{4}{c}{ Population } \\
\cline { 2 - 5 } Sex & Gorge & Siskiyou & Mt. Hood & Tillamook \\
\hline Male & 14 & 10 & 10 & 16 \\
Female & 7 & 4 & 7 & 2 \\
\hline
\end{tabular}


Measurements

Target body regions were digitally photographed and evaluated with the measurement software Image-Pro; after calibrating the photographs for scale, this program is capable of making linear measurements as well as calculating the area of a traced polygon. The inflexible carapace of a spider is generally used as a proxy for size (cf. Macías-Hernández (2008), Delaney (2007), Persons and Uetz (2005), Stratton (2005), Uetz et al (2002), Marshall and Gittleman (1994), and Hagstrum (1971)). I took four different carapace measurements: maximum carapace width, carapace length, carapace area, and distance between posterior lateral eyes. Carapace width was the dimension where alignment error was most apparent, and thus easy to correct. Measurement of total carapace area has the benefit of eliminating any morphometric differences of the carapace between individuals by considering the entire carapace, rather than a single dimension. It is, however, not as easy to detect instances of alignment error. For these reasons, I believe carapace width and carapace area to be the most accurate depictions of overall size, and therefore I rely primarily on these measurements for my conclusions.

I measured two aspects of the males' enlarged tibia. The first is the outer extent of the setae fringing the upper and lower margins of the tibia. These setae effectively extend the apparent surface area of the underlying integument. The other tibia aspect I measured was the area of the tibia integument, not including the 
setae. When considering the overall size of the ornament, the integument plus setae combined is hereafter referred to as the "apparent" size of the tibia, whereas the area of the integument alone is called the "actual" size of the tibia. If the overall size of the ornament is the most important character being judged, the setae are essentially extensions that enlarge the border of the actual integument.

To describe the outer area of the tibia (the "apparent" area), I measured the maximum width of the tibia (including the setae), and the total area of the tibia (again, including setae). To describe the inner area (the "actual" area), I measured the width of the integument of the tibia (not including the setae), and the area of the integument (not including the setae). I also measured the maximum length of the tibia. I believe tibia area (either outer "apparent" area or inner "actual" area) to be the most accurate representation of tibia size because the irregular shape of the ornament causes linear measurements to be less reliable.

Morphometric Photography

I took all photographs with a Canon EOS 30D digital SLR camera through a Leica MZ9.5 stereomicroscope with a Leica 2.5x/SLR Photoprojective using a Leica 10445930 1.0x conversion tube and a Bower brand T-mount camera adapter for Canon EOS. Each spider was photographed at the following angles:

1. Top view of the cephalothorax 
2. Left front of the tibia (with the edge of the setae and edge of the tibia integument visible and in focus; if the setae were especially disheveled they were combed with a fine pin)

Morphometric Measurements

I calibrated the entire camera and stereomicroscope setup with a micrometer and the software program Image-Pro by Media Cybernetics, correlating the magnification of each image taken to the actual area enclosed in the photograph. Every measurement was repeated three times; the resulting data were exported to Microsoft Excel. Within Excel, each of the three replicate measurements were averaged and collated into a single *.csv data sheet for statistical analysis. The following measurements were taken:

1. Tibia:

a. Outer (apparent) area, tracing the outer edge of setae fringe (Not applicable to females)

b. Inner (actual) area, tracing edge of tibia integument itself, without including setae

c. Central length of the tibia, proximal to distal

d. Maximum width of tibia itself, without including setae

e. Maximum width of tibia with setae fringe included (Not applicable to females) 
2. Cephalothorax:

a. Carapace area

b. Carapace length (Down the center line, between the two principal eyes)

c. Maximum carapace width (In all populations this is roughly the center point where the second legs emerge from the cephalothorax)

d. Maximum width between posterior lateral eyes (Measured from the outer edge of eyes)

Tibia measurements that traced the outer edge of the setae fringe were only applicable to males; females do not have analogous structures.

Statistical Analyses

I used one-way ANOVAs to test for significant differences in overall body size between populations and among groups of populations. Tukey's Honestly Significant Difference (HSD) tests were used to determine which populations were significantly different from each other. For comparisons of sexual ornaments, ANCOVAs were used to factor out the covariate (e.g., overall body size) as well as possible interactions between populations and the covariate. Unnecessary predictors were eliminated using a hybrid approach combining stepwise and Akaike's information criterion (AIC)-based model-reducing procedures. If the test revealed significant differences between populations, orthogonal contrasts based on a priori hypotheses 
were tested to extract further information regarding the specific interpopulation relationships. All analyses were performed in the R statistical package.

\section{RESULTS}

Overall Size

One-way ANOVA tests revealed highly significant differences in all four measures of male carapace size among the spider populations ( $P$-values for all ANOVA tests were below 0.001). All patterns of significance from the Tukey's HSD tests were identical among all four measures of carapace size: males from the two beige morph populations (Gorge and Siskiyou) were not significantly different, males from the two bronze morph populations (Mt. Hood and Tillamook) were not significantly different, but both beige morph populations were significantly different from both bronze morph populations (Table 4.2). Figure 4.1 shows the mean maximum carapace width of male spiders from all four populations. Relationships among the remaining three measures of carapace size (carapace length, carapace area, and distance between posterior lateral eyes) were similar and have been omitted here for brevity.

Table 4.2. Adjusted p-values from Tukey's HSD test for pairwise comparisons of all four spider populations for four measures of overall male spider size. Gorge $(n=14)$ and Siskiyou $(n=10)$ populations are beige morphs, Mt. Hood $(n=10)$ and Tillamook $(n=16)$ populations are bronze morphs.

\begin{tabular}{lllll}
\hline \hline & \multicolumn{3}{c}{ Adjusted p-value } \\
\cline { 2 - 4 } Population Comparison & Carapace Width & Carapace Area & Carapace Length & Eye Distance \\
\hline & 115 &
\end{tabular}




\begin{tabular}{lllll} 
Mt. Hood - Gorge & 0.013 & 0.002 & 0.001 & 0.003 \\
Tillamook - Gorge & 0.000 & 0.000 & 0.000 & 0.000 \\
Siskiyou - Gorge & 0.982 & 1.000 & 0.991 & 0.858 \\
Tillamook - Mt. Hood & 0.521 & 0.968 & 0.990 & 1.000 \\
Siskiyou - Mt. Hood & 0.010 & 0.006 & 0.005 & 0.044 \\
Siskiyou - Tillamook & 0.000 & 0.000 & 0.001 & 0.016 \\
\hline
\end{tabular}

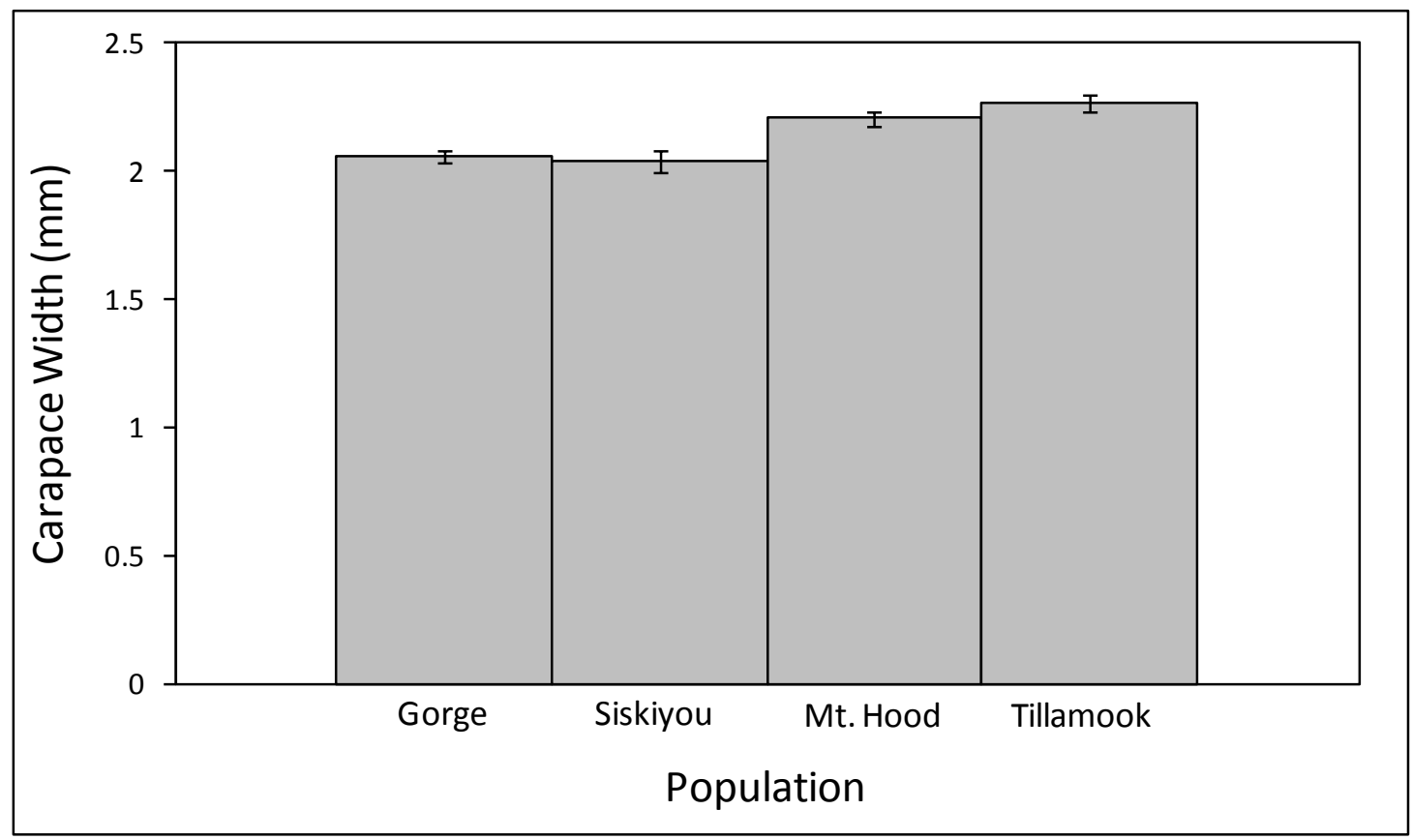

Figure 4.1. Mean carapace width of male spiders. Gorge $(n=14)$ and Siskiyou $(n=10)$ populations are beige morphs, Mt. Hood $(n=10)$ and Tillamook $(n=16)$ populations are bronze morphs. Error bars are standard error.

Grouping the populations by morph for overall body size comparisons appears to be a justified given the results of my analyses (Table 4.2); note that the small sample size for females precludes comparisons of individual populations. Oneway ANOVA tests revealed highly significant differences in mean maximum carapace width as well as in the other three measures of carapace size evaluated among the spider groupings by morph (P-values for all ANOVA tests were below 0.001). Tukey's 
HSD test was used to determine which sexes from each morph were significantly different from each other. All relevant comparisons were significant except the distance between posterior lateral eyes between beige morph females and bronze morph females (Table 4.3). Figure 4.2 shows the mean maximum carapace width of male and female spiders with populations grouped by morph. Relationships among the remaining three measures of carapace size (carapace length, carapace area, and distance between posterior lateral eyes) were similar and have been omitted here for brevity.

Table 4.3. Adjusted p-values from Tukey's HSD test for pairwise comparisons of both male and females spiders grouped by morph for four measures of overall spider size. Sample sizes: beige male = $\underline{24}$, bronze male $=26$, beige female $=11$, bronze female $=9$.

\begin{tabular}{lcccc} 
& \multicolumn{4}{c}{ Adjusted p-value } \\
\cline { 2 - 5 } Morph/Sex Comparison & $\begin{array}{c}\text { Carapace } \\
\text { Width }\end{array}$ & $\begin{array}{c}\text { Carapace } \\
\text { Area }\end{array}$ & $\begin{array}{c}\text { Carapace } \\
\text { Length }\end{array}$ & $\begin{array}{c}\text { Eye } \\
\text { Distance }\end{array}$ \\
\hline Beige Male - Beige Female & 0.009 & 0.023 & 0.006 & 0.000 \\
Bronze Female - Beige Female & 0.018 & 0.004 & 0.005 & 0.097 \\
Bronze Male - Beige Male & 0.000 & 0.000 & 0.000 & 0.000 \\
Bronze Male - Bronze Female & 0.039 & 0.016 & 0.016 & 0.003 \\
\hline
\end{tabular}




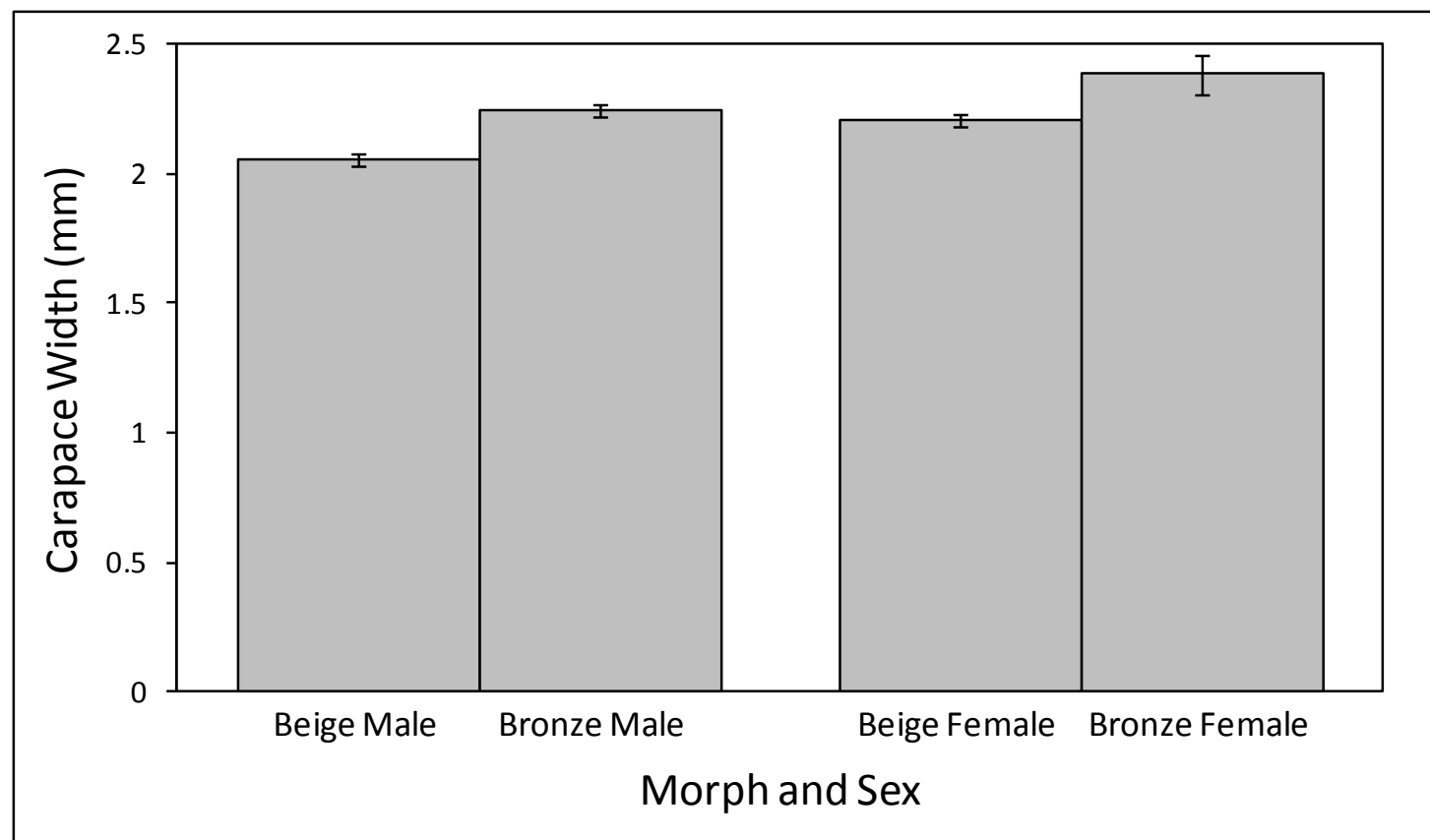

Figure 4.2. Mean carapace width of male and female spiders. Sample sizes: beige male $=24$, bronze male $=26$, beige female $=11$, bronze female $=9$. Error bars are standard error .

Tibia Size

One-way ANOVA tests revealed highly significant differences in all five measures of male tibia size among the spider populations (P-values for all ANOVA tests were below 0.001). All patterns of significance from the Tukey's HSD tests were identical among all five measures of tibia size: males from the two beige morph populations (Gorge and Siskiyou) were not significantly different, males from the two bronze morph populations (Mt. Hood and Tillamook) were not significantly different, but all beige morph populations were significantly different from all bronze morph populations (Table 4.4). Figure 4.3 shows the mean "apparent" tibia area of male spiders from all four populations. Relationships among the remaining four measures 
of tibia size ("actual" tibia area, "apparent" tibia width, "actual" tibia width, and tibia

length) were similar and have been omitted here for brevity.

Table 4.4. Adjusted p-values from Tukey's HSD test for pairwise comparisons of all four spider populations for five measures of male tibia size. Gorge $(n=14)$ and Siskiyou $(n=10)$ populations are beige morphs, Mt. Hood $(n=10)$ and Tillamook $(n=16)$ populations are bronze morphs.

\begin{tabular}{lccccc}
\hline \hline & \multicolumn{5}{c}{ Adjusted p-value } \\
\cline { 2 - 6 } Population Comparison & $\begin{array}{c}\text { "Apparent" } \\
\text { Tibia Area }\end{array}$ & $\begin{array}{c}\text { "Actual" } \\
\text { Tibia Area }\end{array}$ & $\begin{array}{c}\text { "Apparent" } \\
\text { Tibia Width }\end{array}$ & $\begin{array}{c}\text { "Actual” Tibia } \\
\text { Width }\end{array}$ & $\begin{array}{c}\text { Tibia } \\
\text { Length }\end{array}$ \\
\hline Mt. Hood - Gorge & 0.000 & 0.000 & 0.000 & 0.000 & 0.000 \\
Tillamook - Gorge & 0.000 & 0.000 & 0.000 & 0.000 & 0.000 \\
Siskiyou - Gorge & 0.850 & 0.449 & 0.876 & 0.934 & 0.479 \\
Tillamook - Mt. Hood & 0.997 & 0.959 & 0.953 & 0.992 & 0.822 \\
Siskiyou - Mt. Hood & 0.000 & 0.000 & 0.000 & 0.000 & 0.000 \\
Siskiyou - Tillamook & 0.000 & 0.000 & 0.000 & 0.000 & 0.000 \\
\hline
\end{tabular}

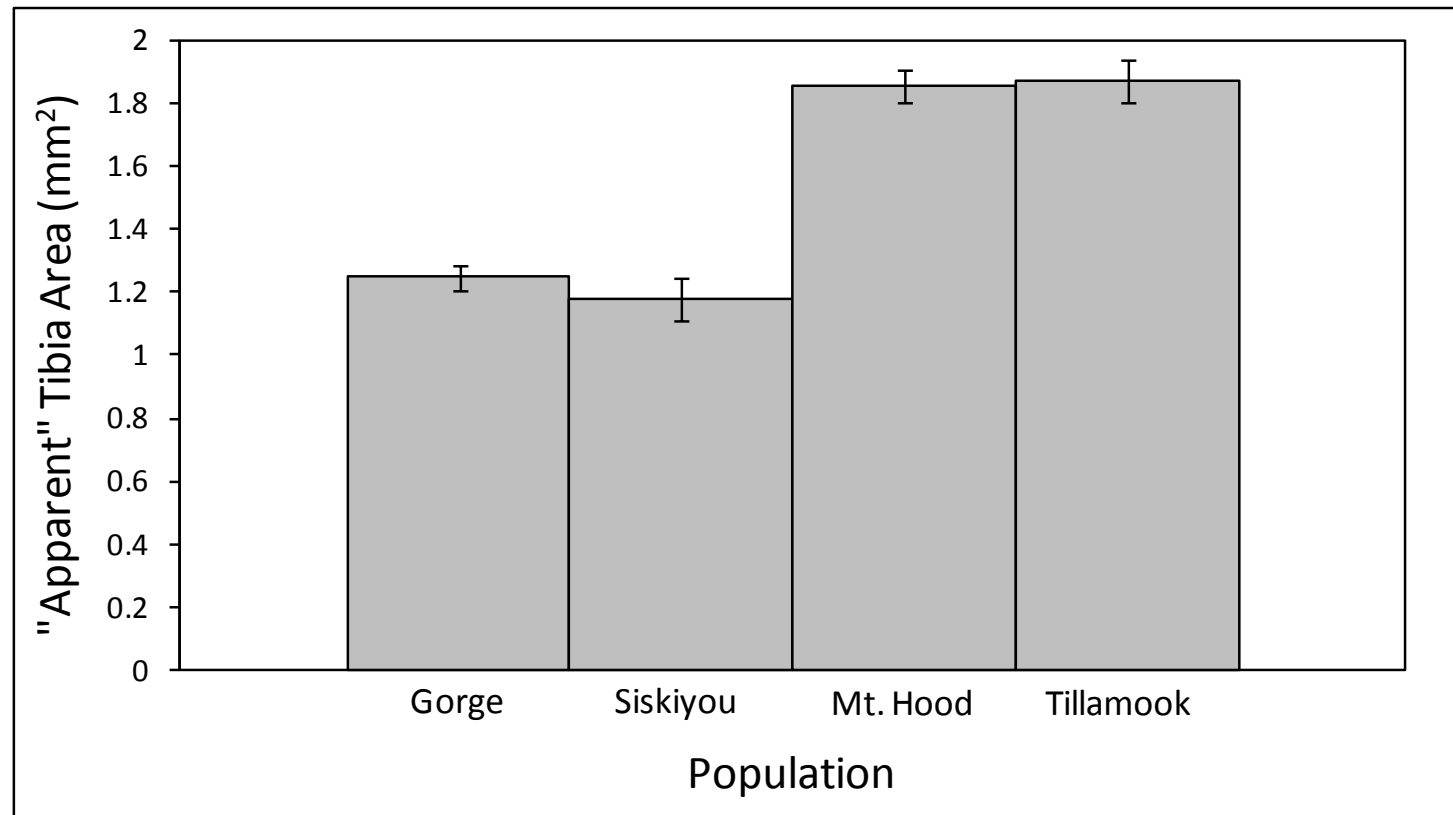

Figure 4.3. Mean "apparent" tibia area of male spiders grouped by population. Gorge $(n=14)$ and Siskiyou $(n=10)$ populations are beige morphs, Mt. Hood $(n=10)$ and Tillamook $(n=16)$ populations are bronze morphs. Error bars are standard error. 
The grouping of Tillamook and Mt. Hood populations into one group, and Siskiyou and Gorge populations into a second, appeared justified given the results of my analyses (Table 4.4) (note again that the small sample size for females precludes comparisons of individual populations). One-way ANOVA tests revealed highly significant differences in all three measures of tibia size that did not include setae among the spider populations ( $P$-values for all ANOVA tests were below 0.001). A Tukey's HSD test revealed that "actual" tibia area, "actual" tibia width and tibia length were not significantly different between bronze morph females and beige morph females (Table 4.5). All other relevant comparisons of "actual" tibia area, "actual" tibia width and tibia length were significant. Student's $t$-tests were used to compare bronze male and beige male "apparent" tibia area and "apparent" tibia width; the results of both were significant (Table 4.5). Figure 4.4 shows the mean "actual" tibia area (not including setae) of male and female spiders with populations grouped by morph. Relationships between the remaining two measures of tibia size that don't include setae ("actual" tibia width and tibia length) were similar and have been omitted here for brevity. Figure 4.5 shows the mean "apparent" tibia area ("actual" tibia width plus tibial fringe of setae); note that females do not have tibial fringes and thus cannot be included in this comparison. The results of the only other measure of tibial size that includes setae ("apparent" tibia width) were similar to that of "apparent" tibia area and have thus been omitted for brevity. 
Table 4.5. Adjusted p-values from Tukey's HSD test for pairwise comparisons of both male and females spiders grouped by morph for five measures of spider tibia size. Sample sizes: beige male $=$ 24 , bronze male $=26$, beige female $=11$, bronze female $=9$. * "Apparent" and "Actual" tibia area $p$ values are results of a $t$-test rather than ANOVA and Tukey's HSD; females do not possess tibial fringe, leaving only one comparison between males of the two morphs.

\begin{tabular}{lccccc}
\hline \hline & \multicolumn{5}{c}{ Adjusted p-value } \\
\cline { 2 - 6 } Morph/Sex Comparison & $\begin{array}{c}\text { "Apparent" } \\
\text { Tibia Area* }\end{array}$ & $\begin{array}{c}\text { "Actual" } \\
\text { Tibia Area }\end{array}$ & $\begin{array}{c}\text { "Apparent" } \\
\text { Tibia Width* }\end{array}$ & $\begin{array}{c}\text { "Actual" } \\
\text { Tibia Width }\end{array}$ & $\begin{array}{c}\text { Tibia } \\
\text { Length }\end{array}$ \\
\hline Beige Male - Beige Female & - & 0.000 & - & 0.000 & 0.001 \\
Bronze Female - Beige Female & - & 0.205 & - & 0.500 & 0.113 \\
Bronze Male - Beige Male & 0.000 & 0.000 & 0.000 & 0.000 & 0.000 \\
Bronze Male - Bronze Female & - & 0.000 & - & 0.000 & 0.000 \\
\hline
\end{tabular}

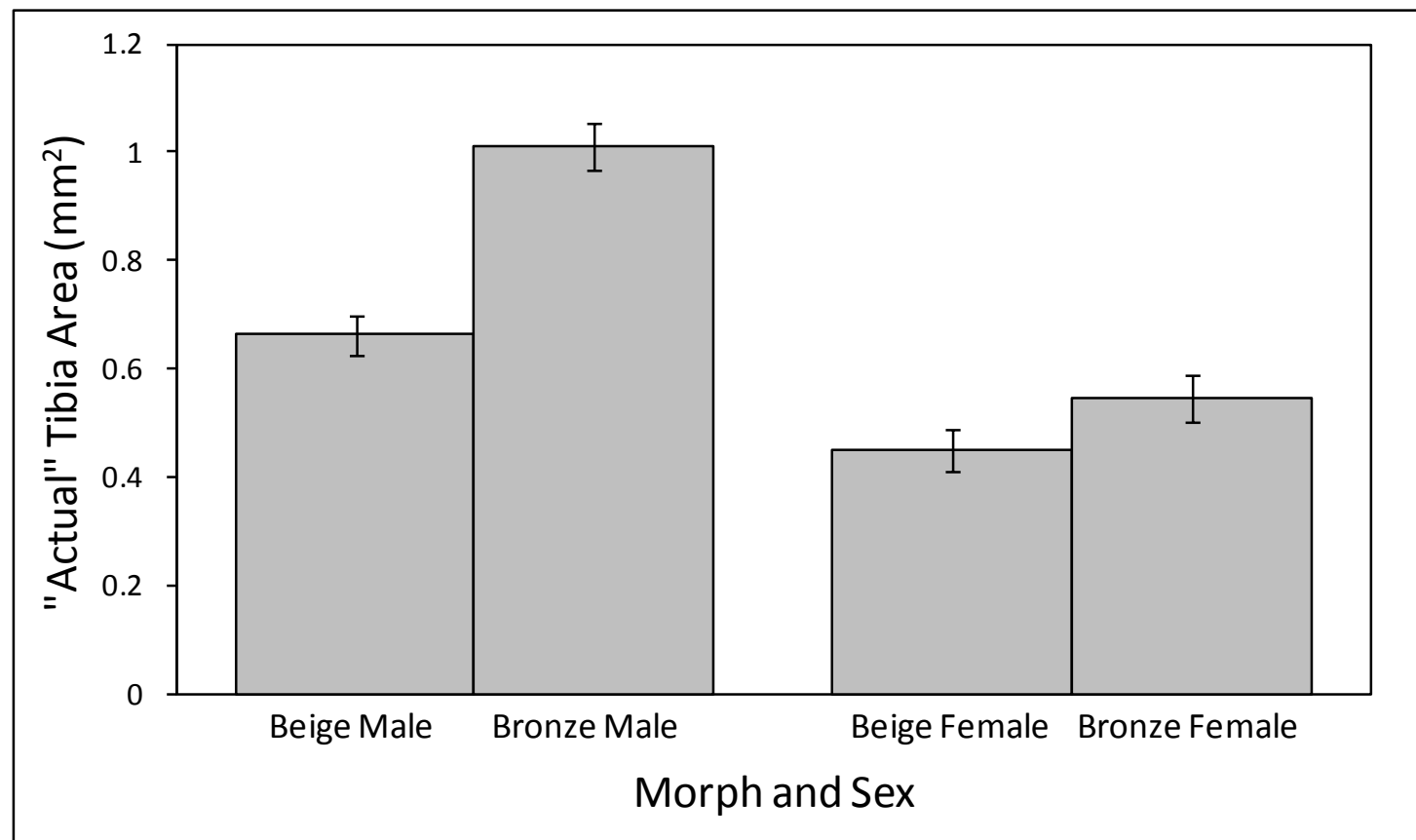

Figure 4.4. Mean "actual" tibia area of male and female spiders grouped by morph. Sample sizes: beige male $=24$, bronze male $=26$, beige female $=11$, bronze female $=9$. Error bars are standard error. 


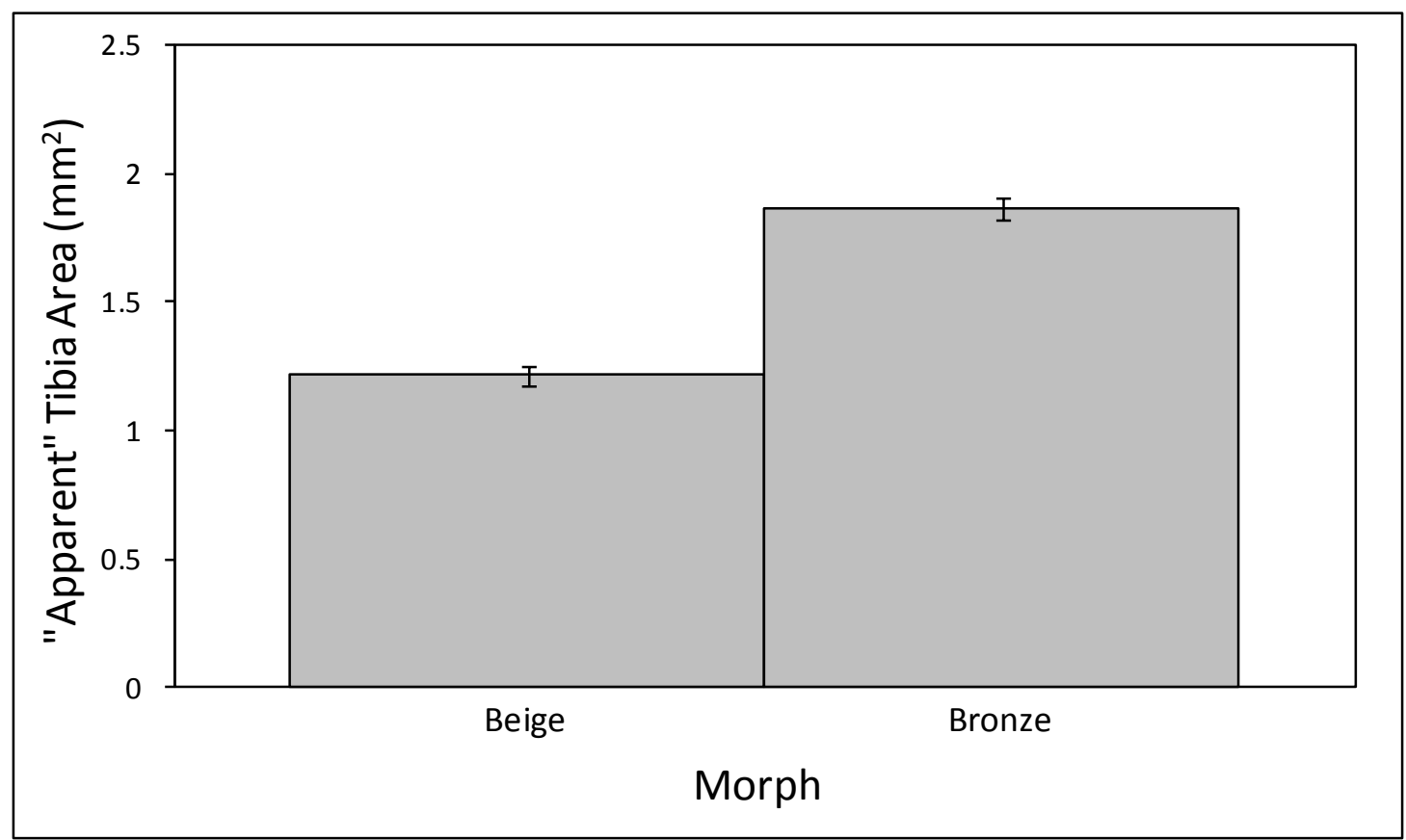

Figure 4.5. Mean "apparent" tibia area of male spiders grouped by morph. Sample sizes: beige male = 24, bronze male = 26; females do not have tibial fringes and thus cannot be included in this comparison. Error bars are standard error.

Male Tibia Size Relative to Overall Size

In order to test male tibia size relative to overall size (that is, factor out size as a covariate), I performed an ANCOVA. The full model of "apparent" tibia area (the response variable) was evaluated against all four of the populations (the factors) and carapace area (the covariate) as well as all possible interactions between population and carapace area. In order to eliminate any unnecessary predictors, this full model was reduced to the minimal adequate model by way of a hybrid approach combining stepwise and AIC-based procedures. This procedure eliminated the interaction of population and carapace area as unnecessary, leaving only the population factor and carapace area covariate to compare to the tibia outer area. This new, minimal 
adequate model was compared to the full model with an ANOVA test to verify that there was no significant difference in explanatory power between the two models; indeed, the resulting $\mathrm{p}$-value was 0.928 .

The removal of the interaction between population and carapace area means that the differences in slopes of "apparent" tibia area versus carapace area are not significantly different. However, an ANCOVA of the minimal adequate model reveals that there are highly significant differences in intercept between one or more of the populations $(p<0.001)$. In order to determine which of the populations or groups of populations are different, I developed three orthogonal contrasts based on the hypotheses that that there is no significant difference in tibia size (corrected for overall body size) between populations of the same morph but that there is a significant difference in size between the populations when grouped by morph. I contrasted Gorge population with Siskiyou population ( $p=0.190$, not significantly different), Mt. Hood population with Tillamook population ( $p=0.816$, not significantly different), and Gorge population plus Siskiyou population with Mt. Hood population plus Tillamook population ( $p<0.001$, highly significantly different). This means that bronze morph males have proportionally larger tibia than beige morph males. Figure 4.6 is a graphical representation of the relationship between "apparent" tibia area and carapace area of the four populations. 


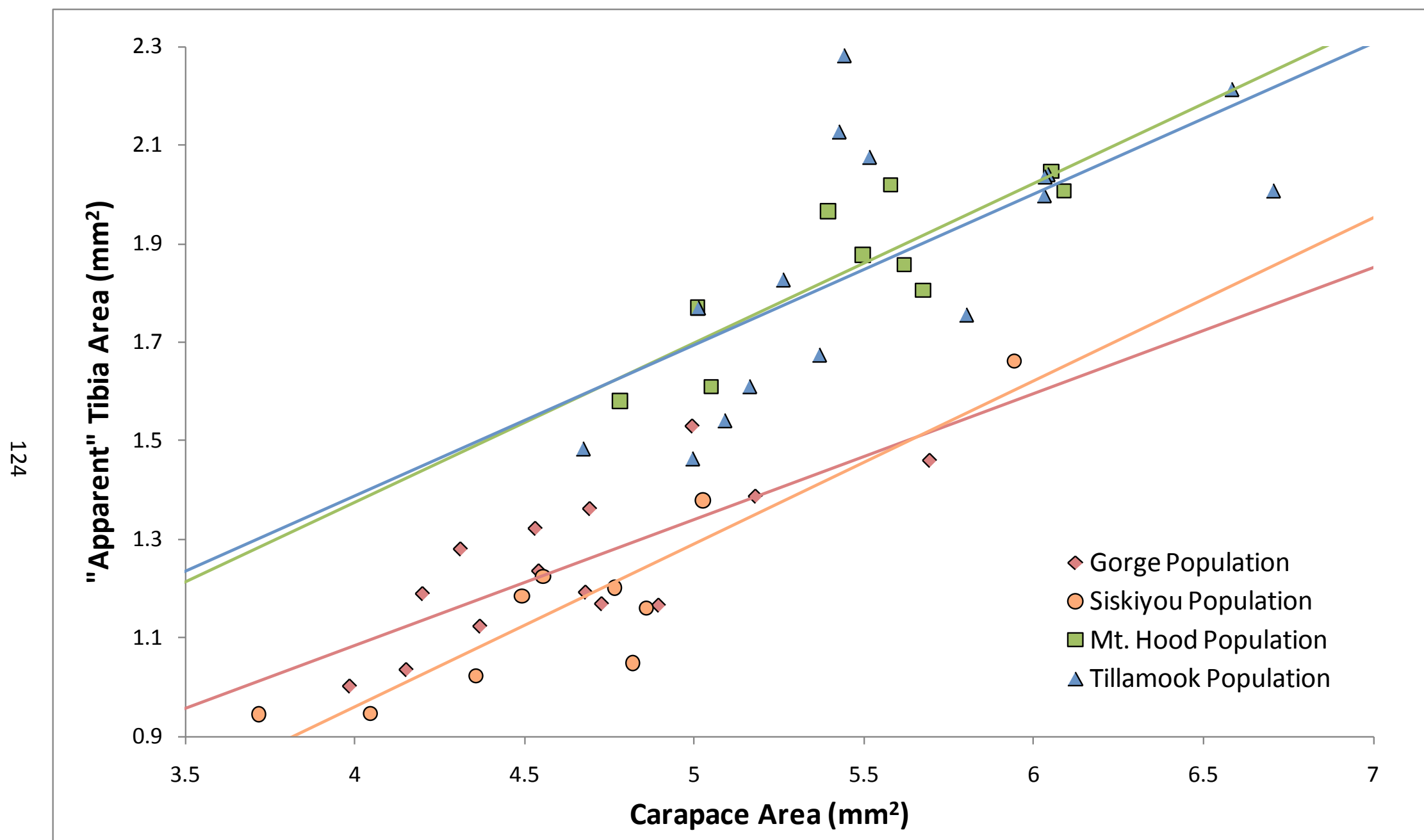

Figure 4.6. Area of male tibia including setae fringe compared to carapace size, sorted by population, including trendlines. Gorge $(n=14)$ and Siskiyou $(n=10)$ populations are beige morphs, Mt. Hood $(n=10)$ and Tillamook $(n=16)$ populations are bronze morphs. 
I also ran an ANCOVA on the similar comparison of "actual" tibia area versus carapace area among the four populations. The minimal adequate model for this comparison excluded interactions among the factors and the covariates (as was the case with the initial comparison of outer tibia area to carapace area), again leaving the predictor variable (tibia size) to be compared against the factors (population) plus a single covariate (carapace size). Also consistent with the previous analysis, the Mt. Hood and Tillamook populations were not significantly different, and the Gorge plus Siskiyou versus Mt. Hood plus Tillamook were significantly different. However, unlike the previous analysis, the Gorge population was significantly different from the Siskiyou population in this analysis. The final output from the contrast (using the same comparisons as the previous analysis) was: Gorge population versus Siskiyou population ( $p=0.018$, significantly different), Mt. Hood population versus Tillamook population ( $p=0.820$, not significantly different), and Gorge population plus Siskiyou population versus Mt. Hood population plus Tillamook population $(p<0.001$, highly significantly different). Figure 4.7 is a graphical representation of the relationship between "actual" tibia area and carapace area of the four populations. This figure shows that the "actual" tibia area adjusted for carapace area of the Mt. Hood and Tillamook population males were larger than the adjusted "actual" tibia areas of both the Gorge and the Siskiyou population males. It also shows that the male Gorge population adjusted "actual" tibia areas were for the most part larger than the male 
Siskiyou population tibia, though the tibia size of the Siskiyou population males increased with body size at a faster rate than that of the Gorge population males. 


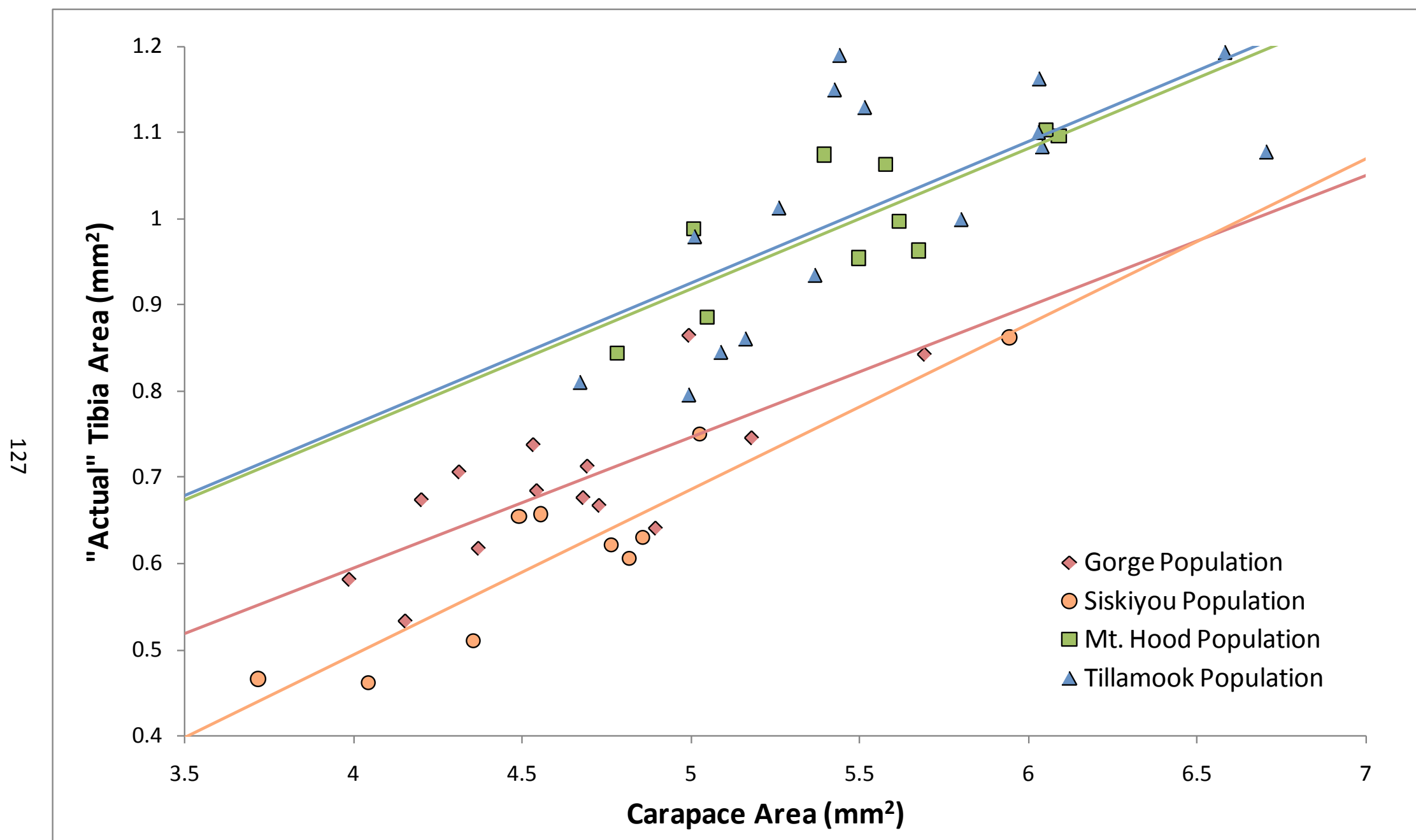

Figure 4.7. Area of male tibia not including setae fringe compared to carapace size, sorted by population, including trendlines. Gorge $(n=14)$ and Siskiyou $(n=10)$ populations are beige morphs, Mt. Hood $(n=10)$ and Tillamook $(n=16)$ populations are bronze morphs. 
Male Tibial Fringe Size Relative to Tibial Integument Size

I compared the ratio between the area of the tibial integument and the area of the fringe of setae surrounding the tibial integument of each population, as well as the male tibia size versus overall size, with an ANCOVA. The full model of tibial fringe (the response variable) was evaluated against all four of the populations (the factors) and "actual" tibia area, i.e., integument only (the covariate) as well as all possible interactions between population and "actual" tibia area. In order to eliminate any unnecessary predictors, this full model was reduced to the minimal adequate model by way of a hybrid approach combining stepwise and AIC-based procedures. This procedure eliminated the interaction of population and inner tibia area as unnecessary, leaving only the population factor and "actual" tibia area covariate to compare to the tibial fringe area. This new, minimal adequate model was compared to the full model with an ANOVA test to verify that there was no significant difference in explanatory power between the two models; the resulting $p$-value was nonsignificant at 0.422 .

The removal of the interaction between population and "actual" tibia area means that the differences in slopes of the tibial fringe area versus "actual" tibia area are not significantly different. However, an ANCOVA of the minimal adequate model reveals that there are highly significant differences in intercept between one of more of the populations $(p<0.001)$. In order to determine which of the populations or groups of populations are different, I developed three orthogonal contrasts based on 
the hypotheses that that there is no significant difference in tibial fringe area (corrected for "actual" tibia size) between populations of the same morph but that there is a significant difference in size between the populations grouped by color morph. I contrasted Gorge population with Siskiyou population ( $p=0.009$, significantly different), Mt. Hood population with Tillamook population $(p=0.210$, not significantly different), and Gorge population plus Siskiyou population with Mt. Hood population plus Tillamook population ( $p=0.446$, not significantly different). This means that Siskiyou population males have proportionally more tibial fringe area than Gorge population males, but that this difference is not significant when the two populations are combined and compared with the males from the two bronze morph populations. Figure 4.8 is a graphical representation of the relationship between "apparent" tibia area and carapace area of the four populations. 


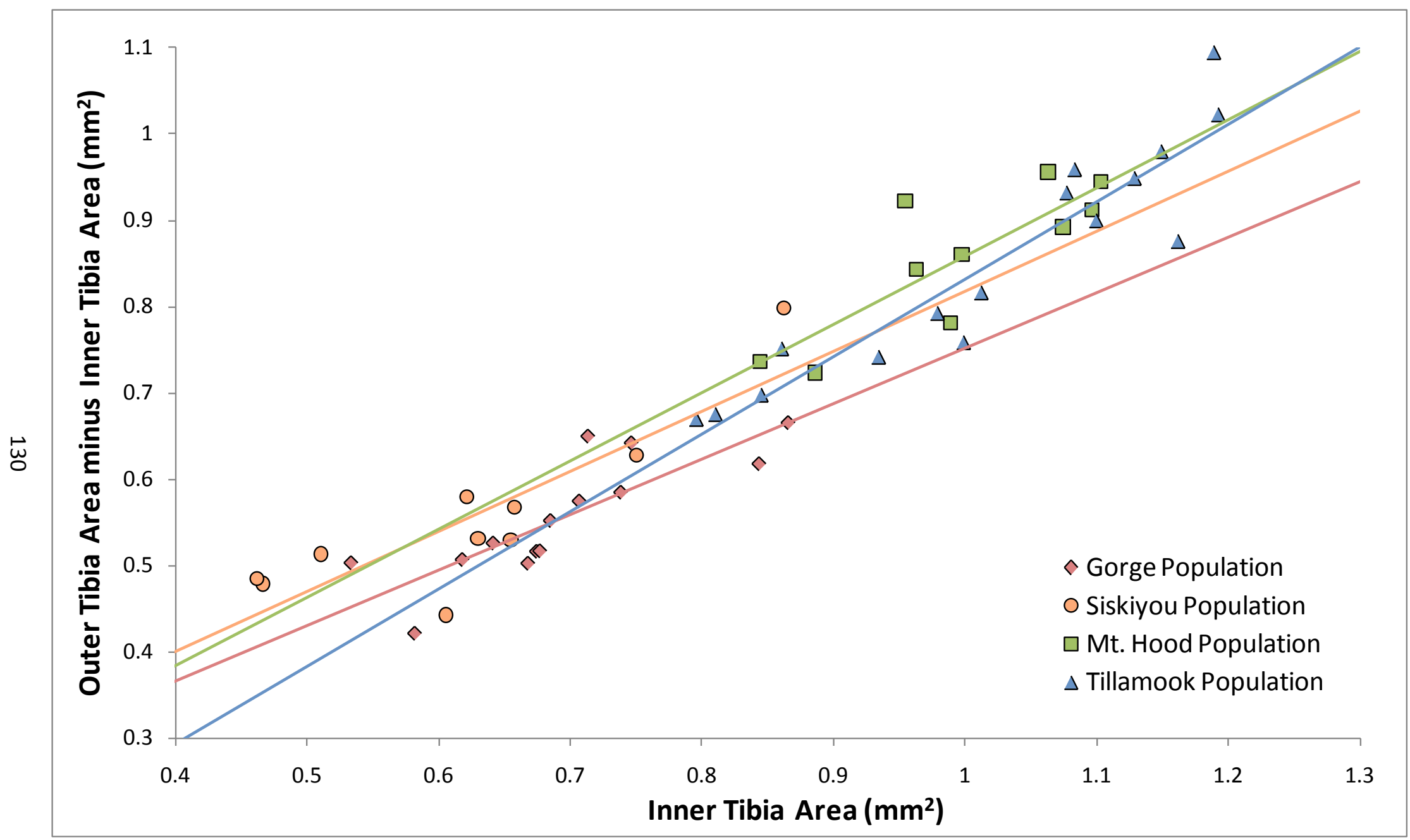

Figure 4.8. Area of male tibia (setae fringe only) compared to tibia area (integument only, not including setae), sorted by population, including trendlines. Gorge $(n=14)$ and Siskiyou $(n=10)$ populations are beige morphs, Mt. Hood $(n=10)$ and Tillamook $(n=16)$ populations are bronze morphs. 
I also ran an ANCOVA on the similar comparison of tibial fringe width ("apparent" tibia width minus "actual" tibia width) versus "actual" tibia width among the four populations; the conclusions were identical with those of tibia integument area versus the area of the tibial fringe: the Siskiyou population males were found to have proportionally wider tibial fringe than Gorge population males, though the difference is not significant when the two populations are combined and compared with the males from the two bronze morph populations. The minimal adequate model excluded interactions between population and "actual" tibia area (as with the previous analysis), and the final results from the contrast (using the same comparisons as the previous analysis) were: Gorge population versus Siskiyou population ( $p=0.018$, significantly different), Mt. Hood population versus Tillamook population ( $p=0.587$, not significantly different), and Gorge population plus Siskiyou population versus Mt. Hood population plus Tillamook population ( $p=0.205$, not significantly different).

Classification of Individuals based on Morphology and Color I performed a jackknifed discriminant function analysis to check whether the morphological and color data I collected could classify male $H$. oregonensis to their respective population or, less specifically, their color morph. The morphological characters I used were "actual" first tibia area, the area of the setae fringe on the first tibia, and carapace area. I used segment classification values (Endler, 1990) of 
all the color characters that I collected: hue, chroma, and brightness for face, cephalothorax, abdomen, and ventrum. I omitted all specimens from the analysis that lacked values for any of these characters; this left me with nine males from the Gorge population, six males from the Siskiyou population, eight male from the Mt. Hood population, and sixteen males from the Tillamook population. Gorge population males were classified correctly $66.67 \%$ of the time, Siskiyou population males were classified correctly $33.33 \%$ of the time, Mt. Hood population males were classified correctly $25.00 \%$ of the time, and Tillamook population males were classified correctly $68.75 \%$ of the time. Note, however, that all misclassifications were for populations of the same color morph. I verified this finding by running a jackknifed discriminant function analysis classifying the specimens solely by color morph. In this analysis, all specimens were classified correctly $100 \%$ of the time.

\section{DISCUSSION}

I demonstrated with the results of the morphometric analysis that the Gorge and Siskiyou population males are not significantly different in body size, and that the Mt. Hood and Tillamook population males are not significantly different in body size. Males from the Gorge and Siskiyou populations were, however, significantly smaller than males of the Mt. Hood and Tillamook populations. Although limited sample size required grouping size measurements of females by color morph rather than comparing individual populations, beige morph females were shown to be 
significantly smaller than bronze morph females. There are two possible reasons for such a disparity in size among the populations. The first is due to natural selection; extrinsic characteristics of the bronze morph population environments could select for larger body size (Nagel and Schluter, 1998). If locally available prey are different than those available in beige morph population environments, specialized morphological adaptations (including size) might result. There also could be adaptive differences in optimal clutch sizes, which would have a strong effect on overall female size (Ford and Seigel, 1989). This could, in turn, affect overall male size by way of gene linkage.

Sexual selection for larger males is another possible cause of an increase in body size. Territoriality among males is strong, and larger body size may confer benefits in intrasexual competition. Female size could increase in step with male size through gene linkage. Explaining this size disparity with sexual selection and gene linkage seems less likely than a natural selection scenario due to the fact that females are already larger than males of their own morph - if the males were the sex benefiting from a larger size, one would expect them to be equal or larger than the females. Presumably, selection favors small rather than large males - this could be explained if molting to adulthood earlier (and thus at a smaller size) allowed males to secure better territories or gave them exclusive access to newly molted females (Zonneveld, 1996). 
First leg tibia size in males of the Gorge and Siskiyou populations were not significantly different, nor were tibia sizes of the Mt. Hood and Tillamook populations significantly different. As with overall body size, however, the tibia of males of Mt. Hood and Tillamook populations were significantly larger than those of the Gorge and Siskiyou populations. What is surprising about these data is the magnitude of difference in male tibia size between Mt. Hood and Tillamook population individuals compared to Gorge and Siskiyou population individuals - Mt. Hood and Tillamook population males have much larger tibia than Gorge and Siskiyou population males, even when considering their larger overall body size. This trend is apparent when overall body size was factored out using ANCOVAs.

A comparison of male first tibia "apparent" area (including the tibial fringe of setae) in which body size is factored out shows no significant difference in size between the Gorge and Siskiyou populations, nor does it show a significant difference between the Mt. Hood and Tillamook populations. However, there is a pronounced difference in proportional size between the populations when the populations are grouped by color morph. A comparison of male first tibia that does not include the tibial fringe of setae (integument area only) returns a slightly different result: as with the "apparent" tibia area comparison, there is no significant difference between the two bronze morph populations, but unlike the previous comparison there is a significant difference between the two beige morph populations. Also like the "apparent" tibia area comparisons, when the populations 
are grouped by color morph there is a significant difference between tibia integument area of beige morph males and bronze morph males.

The fact that there is any significant difference at all in male $H$. oregonensis tibia size after factoring out overall body size among any of the populations or morphs of spiders suggests that there may be differences in sexual selection regimes. The substantially larger tibia area after adjustment for body size of the males of the bronze morph populations compared to the males of the beige morph populations may represent an alternate pathway in mate attraction explored by the bronze morph populations. Whereas the beige morph population males have a higher degree of sexual dichromatism on their anterior compared to the bronze morph population males (see chapter 3 ), bronze males may rely more heavily on tibia size than beige males for attracting females.

The fact that there was a discrepancy between the two comparisons regarding whether or not there was a significant difference between tibia size relative to overall body size between the Gorge population and the Siskiyou population suggests that there may be a difference in the ratio between tibia size with and without the tibial fringe. This means that while the overall ("apparent") size of the tibia between the two populations is not significantly different, the underlying integument ("actual") size of the Siskiyou population is significantly smaller than that of the Gorge population compared to the fringe. This was further investigated in the comparison between male tibial fringe area relative to tibial integument area. This 
test returned a significant difference between Gorge population males and Siskiyou population males; Siskiyou population males had greater tibial fringe area than did the Gorge population males when body size was considered in the analysis.

The lack of any significant difference in "apparent" tibia size (including tibial fringe) after factoring out overall body size between the Gorge and Siskiyou populations, and the presence of a significant difference in tibial integument area implies that Siskiyou males are using setae to replace integument to create the same overall amount of surface area for the tibia. However, the fact that the discriminant function analysis was unable to classify male $H$. oregonensis down to their respective population (but was able to classify them to their respective color morph) suggests that if there is any difference in morphology between Gorge and Siskiyou population males (as well as between Mt. Hood and Tillamook population males), it is slight.

The deceptive adornment of an underlying morphological structure with hair or setae, such as what may be occurring with the tibial fringe in $H$. oregonensis, has been employed in taxa as diverse as ungulates and primates to dance flies, with the end result either exaggerating the apparent size of the structure or preventing the accurate assessment of its actual size (Andersson, 1994; Funk and Tallamy, 2000). Females of the courtship role-reversed long-tailed dance fly, Rhamphomyia longicauda, for example, have fringes of setae along the lateral margins of their legs which, when held against pleural extensions of their abdomen, help conceal the actual size of the abdomen; this deceptive signaling serves to prevent males from 
accurately gauging which females would be optimal mating partners (Funk and Tallamy, 2000). In instances of potential deceptive adornment such as those seen here with $H$. oregonensis, natural selection may play a role if, for example, larger integument area is more costly than setae. This may be the case with $\mathrm{H}$. oregonensis if the swollen tibia of males requires a greater amount of resources or increases the risk of an unsuccessful molt (when molting, the spider must draw each leg out from the confines of the old cuticle; the comparatively large size of this distally located ornament may make the shedding process difficult and dangerous). Sexual selection could also play a role here: females may not necessarily gauge overall area of the tibia during courtship - they may actually prefer a specific ratio of integument to setae fringe. Alternatively, the fringe itself may be a quality indicator to $H$. oregonensis females. Such a scenario was found to occur in a different species of dance fly, R. tarsata, where the leg setae of females are accurate signals of fecundity rather than deceptive, as is the case with $R$. longicauda (LeBas et al., 2003).

There is a distinctive grayish color morph of $H$. oregonensis known to occur in California (S. Masta, pers. comm.); if tibial morphology tends to associate with color morph, as seems to be the case here, differences in the ratio of tibial fringe to integument between the Gorge population and the Siskiyou population could mean that one of the populations is showing signs of grading into this third morph. Finally, the fact that the difference in ratio between tibia integument and tibial fringe is relatively small between Gorge and Siskiyou populations may imply some degree of 
plasticity. If, for example, a greater tibial integument size requires more energy investment, it could be that the Siskiyou population had fewer resources available.

These data suggest that there are multiple morphologically distinct groups of H. oregonensis in the Pacific Northwest. Distinct differences in a prominent male sexual ornament (size of the first leg tibia) between populations imply that differences in sexual selection have played a role in this separation. Subtle differences detected in the tibia of males of the Gorge and Siskiyou populations may indicate different strategies for achieving a larger apparent ornament size. 
CHAPTER 5: Conclusions and Future Directions

In this study I have shown a number of morphological differences among populations of the jumping spider Habronattus oregonensis. The Siskiyou population and the Gorge population both have smaller male tibia size and smaller overall body size, while the Mt. Hood population and the Tillamook population both have larger tibia and body size. Additionally, smaller male tibia size and smaller overall body size are associated with the beige color morph while larger tibia and body size are associated with the bronze color morph. It is unclear as to whether geographic boundaries are preventing populations of dissimilar morphotypes from interbreeding with each other, though the scattered distribution of the populations (with one documented instance of populations of different morphotypes being less than $60 \mathrm{~km}$ apart) suggests that the morphotypes are not geographically isolated from each other. Nevertheless, color, body size, and tibia size characters remain largely discrete, with no known intermediates. Additionally, widely separated populations (e.g., the Gorge population and the Siskiyou population - over $420 \mathrm{~km}$ apart) are maintaining similar morphologies despite a lack of obvious geographic connection. Together, these factors imply that selection is maintaining the integrity of the separate morphotypes. Indeed, the apparently complete lack of any such intermediates between the two morphotypes suggests that selection against any such intermediate is strong. The distinctly bimodal distribution in characters examined in this study (and encountered - insofar as morph coloration goes - in past 
surveys of this species) and the lack of any clear environmental correlate to such characters implies that natural selection is not likely the principal driving force behind this segregation of morphologies - a cline of morphological traits associated with an environmental character would be expected if natural selection were the cause of this diversity (Stillwell et al., 2007). Rather, sexual selection seems to be maintaining the integrity of the two morphotypes. Additionally, preliminary analysis of mtDNA sequences suggests recent divergence of the morphotypes (S. Masta, unpubl.). Such a rigorously maintained selection for bimodalism of morphology may be a case of incipient speciation due to sexual selection. This study system is notable in that the divergence between the morphotypes seems to be driven largely by sexual selection, but is at the same time constrained by natural selection.

Despite the similarities in habitat hue and chroma (and presumably predation pressure) among all four sites, there was a surprising amount of difference in relative crypsis of males between the two color morphs. Beige morph males fit the prediction of a cryptic dorsum and noncryptic anterior, while the bronze morph males had a cryptic anterior and a less cryptic abdomen. While the colorimetric deviations from crypsis were quite similar between the morphs, the magnitude and location of these differences were very different. Taken on their own, these hue and chroma differences offer few hints as to what might be causing this pattern. However, when the color analysis is combined with the morphometric analysis, one possibility becomes apparent. It appears that sexual selection is weighted differently 
among different characters of the morphs. Sexual selection seems to have directed bronze morph males away from overall crypsis to comparatively small degree, but they have been subject to selection for a more substantial enlargement of their tibia. It may be that the investment and risk to males incurred by having such large tibia precludes the additional handicap of a dramatic deviation from crypsis, despite female choice - the risk may simply be too great to do both. This possibility of a tradeoff is supported by the females of both morphs, which do not likely experience substantial pressure from sexual selection for differences in morphology or coloration; subject only (or mostly) to natural selection, females of both morphs are comparable in their differences from habitat hue and chroma.

Here I have highlighted the potential for which signaling can affect color and morphology and especially how these two factors may interact in the same organism. However, while these data are suggestive of causality, to conclusively determine whether color and morphology are indeed driven by natural selection and/or sexual selection, further investigation is necessary.

Mating trials under specific light regimens would be an excellent step towards identifying the role of color in mate selection. Female acceptance rates could be evaluated in trials where available light is modified such that target wavelengths are either omitted or increased. It would be particularly interesting if the ambient light of the mating trial could cause one color morph to resemble another (for example, if the bronze morph males were exposed to light supplemented with longer 
wavelengths during mating trials, causing their whitish facial setae to appear red in a similar manner to those of the beige morph males). For mating trials focusing on non-color morphology, males with tibial setae that have been experimentally trimmed or augmented would be telling as well.

Another potentially useful behavioral study would be to stage interactions between males. This could provide information as to whether any morphological trait was associated with success in male-male competitions.

To determine whether color is directed by natural selection, model spiders of varying colorations could be deployed at locations known to harbor populations of the spiders and monitored for number of predator attacks as well as the species of the predators.

Testing for abiotic differences in habitats among populations or morphs of the spider would also be useful. The significant differences found in air temperature at the collection locations are interesting, and merit additional investigation. Anecdotal evidence suggests that bronze morph $H$. oregonensis are found in more northern latitudes, while beige morph (and a third "grey" morph) $H$. oregonensis are found in more southern latitudes (with Oregon appearing to be something of a transitional zone between the bronze morph and the other two morphs) (S. Masta, pers. comm.; Griswold, 1987). The possibility of different optimal temperature ranges between the morphs could be addressed by a more fine-grained analysis of substrate temperatures, especially if the substrates of the spiders could be investigated at 
multiple times of the year throughout the range of spider activity. Additionally, conclusions regarding differences in habitat and geographic extents of morphotypes would be more robust with the inclusion of more sample sites.

The inclusion of more samples sites would greatly inform the investigation of the variation among populations, and come closer to answering the question of how distinct the morphs really are. The results of this study point to a real difference between the bronze morph populations and the beige morph populations, but finerscaled differences among populations within the same morph are less conclusive. Particularly, the Gorge and Siskiyou populations have nearly identical colorations, though the ratio between tibia area and tibial setae of males points to possible distinctions between the two populations. Phylogenetic analysis of these different populations could provide clues as to how much interbreeding is occurring among different sites and particularly between different morphs.

Finally, of course, there is no substitute for a solid familiarity with the natural history of a target organism. The limited knowledge of $H$. oregonensis is a real handicap when attempting to draw even some of the most basic conclusions. While anecdotal evidence from field collections has been extremely useful in filling this void, more time spent studying these organisms in their natural habitat is clearly needed. One natural history unknown that would be particularly useful is where these spiders spend their lives outside of the narrow window of time in which they were collected for this study. The potential for living in two (or more) different 
habitats (with the associated differences in light regime, predator species presence, and prey availability) could have a marked influence on the spiders' overall crypsis. In conjunction with habitat location of the spiders, it would also be useful to perform a more quantitative analysis of juvenile coloration. The (slight) apparent differences in anterior color patterns between juveniles and adult females of both morphs (see chapter 2 results for a qualitative documentation) suggest that there may be some sort of visual cue among conspecifics, perhaps signaling to males whether an individual is sexually mature and thus potentially receptive to courtship. A quantitative analysis of the differences between females and juveniles would be important in evaluating the crypsis of the spiders throughout their entire lifespan, as well as shedding light on the colorimetric differences between immature individuals and adult females. 


\section{REFERENCES}

Andersson, M. B. 1994. Sexual selection. Princeton University Press, Princeton, N.J.

Andersson, S., J. Ornborg, and M. Andersson. 1998. Ultraviolet sexual dimorphism and assortative mating in blue tits. Proceedings of the Royal Society of London Series B-Biological Sciences, 265:445-450.

Andersson, S., and M. Prager. 2006. Quantifying Colors. In G. E. Hill and K. J. McGraw (eds.), Bird Coloration, Volume 1, Mechanisms and Measurements, Vol. 1, pp. 640. Harvard University Press, Cambridge, Mass.

Arnqvist, G., M. Edvardsson, U. Friberg, and T. Nilsson. 2000. Sexual conflict promotes speciation in insects. Proceedings of the National Academy of Sciences of the United States of America, 97:10460-10464.

Backhaus, W. 1991. Color opponent coding in the visual system of the honeybee. Vision Research, 31:1381-1397.

Backhaus, W., and R. Menzel. 1987. Color Distance Derived from a Receptor Model of Color Vision in the Honeybee. Biological Cybernetics, 55:321-331.

Blest, A. D., R. C. Hardie, P. Mclntyre, and D. S. Williams. 1981. The spectral sensitivities of identified receptors and the function of retinal tiering in the principal eyes of a jumping spider. Journal of Comparative Physiology, 145:227-239.

Briscoe, A. D., and L. Chittka. 2001. The evolution of color vision in insects. Annual Review of Entomology, 46:471-510.

Chittka, L. 1992. The colour hexagon: a chromaticity diagram based on photoreceptor excitations as a generalized representation of colour opponency. Journal of Comparative Physiology A: Neuroethology, Sensory, Neural, and Behavioral Physiology, 170:533-543.

Chittka, L., W. Beier, H. Hertel, E. Steinmann, and R. Menzel. 1992. Opponent colour coding is a universal strategy to evaluate the photoreceptor inputs in Hymenoptera. Journal of Comparative Physiology a-Sensory Neural and Behavioral Physiology, 170:545-563.

Chittka, L., A. Shmida, N. Troje, and R. Menzel. 1994. Ultraviolet as a component of flower reflections, and the colour perception of hymenoptera. Vision Research, 34:1489-1508.

Clark, D. L., and G. W. Uetz. 1990. Video image recognition by the jumping spider, Maevia inclemens (Araneae: Salticidae). Animal behaviour, 40:884-890.

Crawford, R. L. 1988. An Annotated Checklist of the Spiders of Washington, Burke Museum contributions in anthropology and natural history. Thomas Burke Memorial Washington State Museum, Seattle, WA.

Cuthill, I. C., A. T. D. Bennett, J. C. Partridge, and E. J. Maier. 1999. Plumage Reflectance and the Objective Assessment of Avian Sexual Dichromatism. American Naturalist, 153:183-200. 
Delaney, K. J., J. A. Roberts, and G. W. Uetz. 2007. Male signaling behavior and sexual selection in a wolf spider (Araneae : Lycosidae): a test for dual functions. Behavioral Ecology and Sociobiology, 62:67-75.

DeVoe, R. D. 1975. Ultraviolet and green receptors in principal eyes of jumping spiders. Journal of General Physiology, 66:193-207.

Eaton, M. D. 2005. Human vision fails to distinguish widespread sexual dichromatism among sexually "monochromatic" birds. Proceedings of the National Academy of Sciences of the United States of America, 102:10942-10946.

Endler, J. A. 1983. Natural and sexual selection on color patterns in poeciliid fishes. Environmental Biology of Fishes, 9:173-190.

-. 1990. On the measurement and classification of colour in studies of animal colour patterns. Biological Journal of the Linnean Society, 41:315-352.

-. 1991. Variation in the appearance of guppy color patterns to guppies and their predators under different visual conditions. Vision Research, 31:587-608.

-. 1992. Signals, signal conditions, and the direction of evolution. American Naturalist, 139:S125-S153.

-. 1993. The color of light in forests and its implications. Ecological Monographs, 63:1-27.

Endler, J. A., and P. W. Mielke. 2005. Comparing entire colour patterns as birds see them. Biological Journal of the Linnean Society, 86:405-431.

Endler, J. A., and M. Théry. 1996. Interacting effects of lek placement, display behavior, ambient light, and color patterns in three neotropical forestdwelling birds. American Naturalist, 148:421-452.

Fleishman, L. K., and M. Persons. 2001. The influence of stimulus and background colour on signal visibility in the lizard Anolis cristatellus. Journal of Experimental Biology, 204:1559-1575.

Ford, N. B., and R. A. Seigel. 1989. Relationships among Body Size, Clutch Size, and Egg Size in Three Species of Oviparous Snakes. Herpetologica, 45:75-83.

Fukushi, T. 1990. Colour discrimination from various shades of grey in the trained blowfly, Lucilia cuprina. Journal of Insect Physiology, 36:69-75.

Funk, D. H., and D. W. Tallamy. 2000. Courtship role reversal and deceptive signals in the long-tailed dance fly, Rhamphomyia longicauda. Animal behaviour, 59:411-421.

Goldsmith, T. H. 1990. Optimization, Constraint, and History in the Evolution of Eyes. Quarterly Review of Biology, 65:281-322.

Green, C. H., and S. Flint. 1986. An analysis of colour effects in the performance of the F2 trap against Glossina pallidipes Austen and G. morsitans morsitans Westwood (Diptera: Glossinidae). Bulletin of Entomological Research, 76:409418. 
Griswold, C. E. 1987. A revision of the jumping spider genus Habronattus F.O.P.Cambridge (Araneae; Salticidae), with phenetic and cladistic analyses. University of California Press, Berkeley.

Hagstrum, D. W. 1971. Carapace Width as a Tool for Evaluating the Rate of Development of Spiders in the Laboratory and the Field. Annals of The Entomological Society of America, 64:757-760.

Hart, N. S. 2001. The visual ecology of avian photoreceptors. Progress in Retinal and Eye Research, 20:675-703.

Hart, N. S., and D. M. Hunt. 2007. Avian visual pigments: Characteristics, spectral tuning, and evolution. American Naturalist, 169:S7-S26.

Hart, N. S., and M. Vorobyev. 2005. Modelling oil droplet absorption spectra and spectral sensitivities of bird cone photoreceptors. Journal of Comparative Physiology A: Neuroethology, Sensory, Neural, and Behavioral Physiology, 191:381-392.

Huber, B. A. 2005. Sexual selection research on spiders: progress and biases. Biological Reviews of the Cambridge Philosophical Society, 80:363-85.

Hurvich, L. M. 1981. Color vision. Sinauer Associates, Sunderland, Mass.

Hyslop, N. P. 2009. Impaired visibility: the air pollution people see. Atmospheric Environment, 43:182-195.

Kirkpatrick, M. 1987. Sexual selection by female choice in polygynous animals. Annual Review of Ecology and Systematics, 18:43-70.

Kwiatkowski, M. A., and B. K. Sullivan. 2002. Geographic Variation in Sexual Selection among Populations of an Iguanid Lizard, Sauromalus obesus (=ater). Evolution, 56:2039-2051.

Lande, R. 1981. Models of Speciation by Sexual Selection on Polygenic Traits. Proceedings of the National Academy of Sciences of the United States of America-Biological Sciences, 78:3721-3725.

Leal, M., and L. J. Fleishman. 2004. Differences in visual signal design and detectability between allopatric populations of Anolis lizards. American Naturalist, 163:26-39.

LeBas, N. R., L. R. Hockham, and M. G. Ritchie. 2003. Nonlinear and correlational sexual selection on 'honest' female ornamentation. Proceedings of the Royal Society of London Series B-Biological Sciences, 270:2159-2165.

LeBas, N. R., and N. J. Marshall. 2000. The role of colour in signalling and male choice in the agamid lizard Ctenophorus ornatus. Proceedings of the Royal Society of London Series B-Biological Sciences, 267:445-452.

Lim, M. L. M., and D. Q. Li. 2006. Extreme ultraviolet sexual dimorphism in jumping spiders (Araneae : Salticidae). Biological Journal of the Linnean Society, 89:397-406. 
-. 2007. Effects of age and feeding history on structure-based UV ornaments of a jumping spider (Araneae : Salticidae). Proceedings of the Royal Society BBiological Sciences, 274:569-575.

Macedonia, J. M. 2001. Habitat light, colour variation, and ultraviolet reflectance in the Grand Cayman anole, Anolis conspersus. Biological Journal of the Linnean Society, 73:299-320.

Macedonia, J. M., A. C. Echternacht, and J. W. Walguarnery. 2003. Color Variation, Habitat Light, and Background Contrast in Anolis carolinensis along a Geographical Transect in Florida. Journal of Herpetology, 37:467-478.

Macedonia, J. M., J. F. Husak, Y. M. Brandt, A. K. Lappin, and T. Baird. 2004. Sexual dichromatism and color conspicuousness in three populations of collared lizards (Crotaphytus collaris) from Oklahoma. Journal of Herpetology, 38:340354.

Macías-Hernández, N., P. Oromí, and M. A. Arnedo. 2008. Patterns of diversification on old volcanic islands as revealed by the woodlouse-hunter spider genus Dysdera (Araneae, Dysderidae) in the eastern Canary Islands. Biological Journal of the Linnean Society, 94:589-615.

Marshall, S. D., and J. L. Gittleman. 1994. Clutch Size in Spiders: Is More Better? Functional Ecology, 8:118-124.

Masta, S. E., and W. P. Maddison. 2002. Sexual selection driving diversification in jumping spiders. Proceedings of the National Academy of Sciences of the United States of America, 99:4442-4447.

McCoy, J. K., S. F. Fox, and T. A. Baird. 1994. Geographic Variation in Sexual Dimorphism in the Collared Lizard, Crotaphytus collaris (Sauria: Crotaphytidae). The Southwestern Naturalist, 39:328-335.

McCoy, J. K., H. J. Harmon, T. A. Baird, and S. F. Fox. 1997. Geographic Variation in Sexual Dichromatism in the Collared Lizard, Crotaphytus collaris (Sauria: Crotaphytidae). Copeia, 1997:565-571.

Menzel, R., and W. Backhaus. 1989. Color Vision Honey Bees: Phenomena and Physiological Mechansims. In D. G. Stavenga and R. C. Hardie (eds.), Facets of vision, pp. x, 454 p. Springer-Verlag, Berlin; New York.

Merbs, S. L., and J. Nathans. 1992. Absorption spectra of human cone pigments. Nature, 356:433-435.

Montgomerie, R. 2006. Analyzing Colors. In G. E. Hill and K. J. McGraw (eds.), Bird Coloration, Volume 1, Mechanisms and Measurements, Vol. 1, pp. 640. Harvard University Press, Cambridge, Mass.

Nagel, L., and D. Schluter. 1998. Body Size, Natural Selection, and Speciation in Sticklebacks. Evolution, 52:209-218.

Paquin, P., D. J. Buckle, N. Dupérré, and C. D. Dondale. 2010. Checklist of the spiders (Araneae) of Canada and Alaska. Zootaxa, 2461:1-170. 
Peaslee, A. G., and G. Wilson. 1989. Spectral sensitivity in jumping spiders (Araneae, Salticidae). Journal of Comparative Physiology A: Neuroethology, Sensory, Neural, and Behavioral Physiology, 164:359-363.

Peckham, G. W., and E. G. Peckham. 1889. Observations on sexual selection in spiders of the family Attidae. Natural History Society of Wisconsin, Milwaukee.

-. 1890. Additional observations on sexual selection in spiders of the family Attidæ, with some remarks on Mr. Wallace's theory of sexual ornamentation. Natural history society of Wisconsin, Milwaukee.

-. 1909. Revision of the Attidae of North America. Democrat Printing Co. State Printer, Madison.

Peitsch, D., A. Fietz, H. Hertel, J. Desouza, D. F. Ventura, and R. Menzel. 1992. The spectral input systems of hymenopteran insects and their receptor-based colour vision. Journal of Comparative Physiology A: Neuroethology, Sensory, Neural, and Behavioral Physiology, 170:23-40.

Persons, M. H., and G. W. Uetz. 2005. Sexual cannibalism and mate choice decisions in wolf spiders: influence of male size and secondary sexual characters. Animal behaviour, 69:83-94.

Richman, D. B. 1982. Epigamic Display in Jumping Spiders (Araneae, Salticidae) and Its Use in Systematics. Journal of Arachnology, 10:47-67.

Schultz, T. D. 2001. Tiger beetle defenses revisited: Alternative defense strategies and colorations of two neotropical tiger beetles, Odontocheila nicaraguensis Bates and Pseudoxycheila tasalis Bates (Carabidae: Cicindelinae). Coleopterists Bulletin, 55:153-163.

Seehausen, O., and J. J. M. van Alphen. 1998. The effect of male coloration on female mate choice in closely related Lake Victoria cichlids (Haplochromis nyererei complex). Behavioral Ecology and Sociobiology, 42:1-8.

Seehausen, O., J. J. M. vanAlphen, and F. Witte. 1997. Cichlid fish diversity threatened by eutrophication that curbs sexual selection. Science, 277:18081811.

Smith, H. 1982. Light Quality, Photoperception, and Plant Strategy. Annual Review of Plant Physiology, 33:481-518.

Solar Radiation Research Laboratory (BMS). 2010. NREL Outdoor Spectral Data Online Database. Resource Information and Forecasting Group; Electricity, Resources, and Building Systems Integration Center; Measurement and Instrumentation Data Center (MIDC); National Renewable Energy Laboratory.

Stillwell, R. C., G. E. Morse, and C. W. Fox. 2007. Geographic variation in body size and sexual size dimorphism of a seed-feeding beetle. American Naturalist, 170:358-369.

Stratton, G. E. 2005. Evolution of ornamentation and courtship behavior in Schizocosa: Insights from a phylogeny based on morphology (Araneae, Lycosidae). Journal of Arachnology, 33:347-376. 
Stuart-Fox, D., A. Moussalli, and M. J. Whiting. 2007. Natural selection on social signals: Signal efficacy and the evolution of chameleon display coloration. American Naturalist, 170:916-930.

Stuart-Fox, D. M., A. Moussalli, G. R. Johnston, and I. P. F. Owens. 2004. Evolution of color variation in dragon lizards: Quantitative tests of the role of crypsis and local adaptation. Evolution, 58:1549-1559.

Stuart-Fox, D. M., and T. J. Ord. 2004. Sexual selection, natural selection and the evolution of dimorphic coloration and ornamentation in agamid lizards. Proceedings of the Royal Society of London Series B-Biological Sciences, 271:2249-2255.

Théry, M., M. Debut, D. Gomez, and J. Casas. 2005. Specific color sensitivities of prey and predator explain camouflage in different visual systems. Behavioral Ecology, 16:25-29.

Uetz, G. W., R. Papke, and B. Kilinc. 2002. Influence of feeding regime on body size, body condition and a male secondary sexual character in Schizocosa ocreata wolf spiders (Araneae, Lycosidae): condition-dependence in a visual signaling trait. Journal of Arachnology, 30:461-469.

Uy, J. A. C., and J. A. Endler. 2004. Modification of the visual background increases the conspicuousness of golden-collared manakin displays. Behavioral Ecology, 15:1003-1010.

Wikelski, M., and F. Trillmich. 1997. Body Size and Sexual Size Dimorphism in Marine Iguanas Fluctuate as a Result of Opposing Natural and Sexual Selection: An Island Comparison. Evolution, 51:922-936.

Yamashita, S., and H. Tateda. 1976. Spectral sensitivities of jumping spider eyes. Journal of Comparative Physiology, 105:29-41.

Zonneveld, C. 1996. Being Big or Emerging Early? Polyandry and the Trade-Off between Size and Emergence in Male Butterflies. The American Naturalist, 147:946-965.

Zschokke, S. 2002. Ultraviolet reflectance of spiders and their webs. The Journal of Arachnology, 30:246-254. 


\section{APPENDIX}

Table A - 1. Student's $t$-test comparing raw brightness values of all spider reflectance spectra sorted by population, sex, and body region as well as habitat reflectance spectra grouped by population. Note that there are no Mt. Hood population leaf reflectance spectra to compare, nor are there enough Tillamook population leaf reflectance spectra to compare. Reflectance data combined with solar irradiance data. Listed below are P-values, $\alpha=0.05$. Table continued on the following pages.

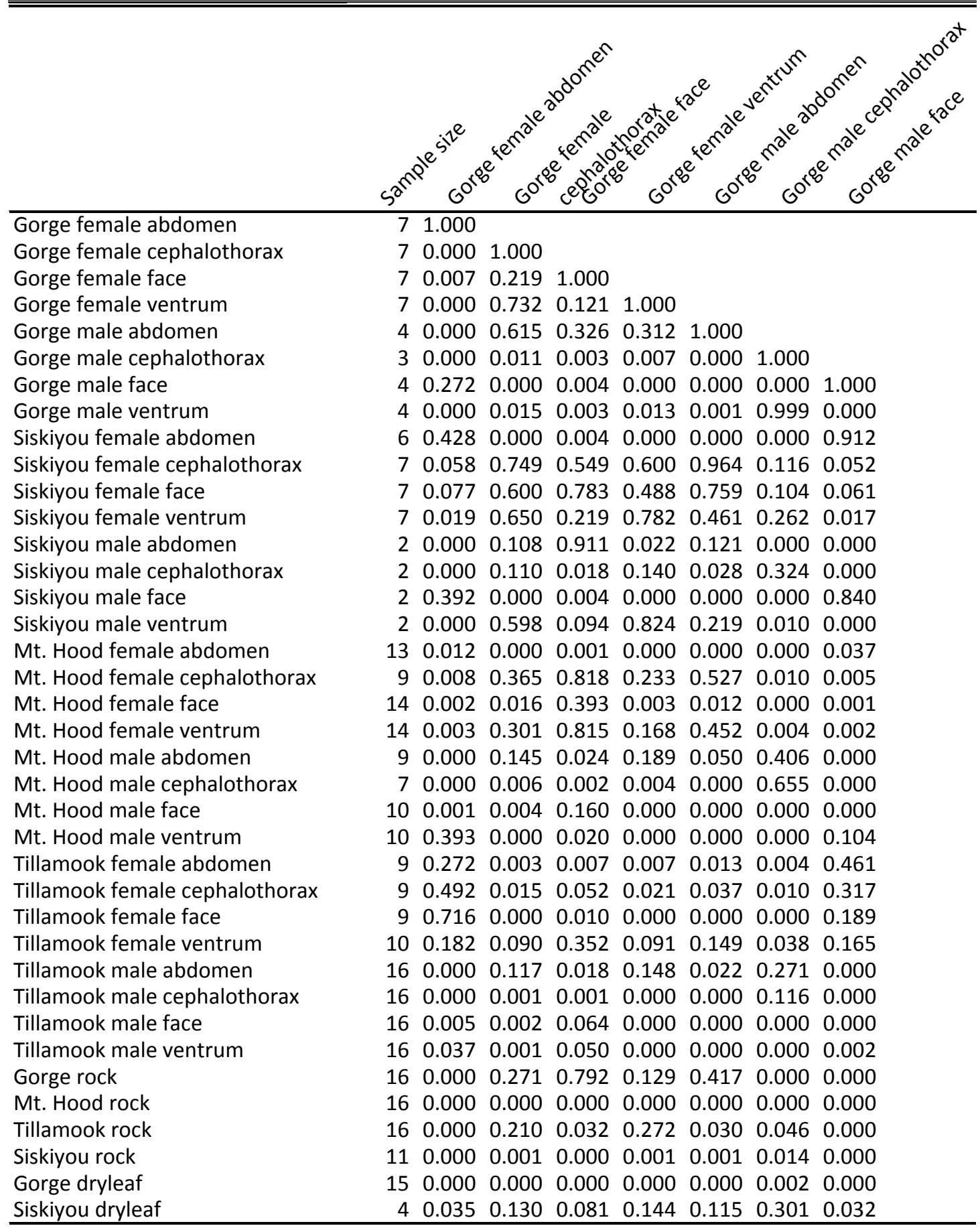


Table A - 1 (continued).

\begin{tabular}{|c|c|c|c|c|c|c|c|}
\hline Gorge female abdomen & 7 & & & & & & \\
\hline Gorge female cephalothorax & 7 & & & & & & \\
\hline Gorge female face & 7 & & & & & & \\
\hline Gorge female ventrum & 7 & & & & & & \\
\hline Gorge male abdomen & 4 & & & & & & \\
\hline Gorge male cephalothorax & 3 & & & & & & \\
\hline Gorge male face & 4 & & & & & & \\
\hline Gorge male ventrum & 4 & 1.000 & & & & & \\
\hline Siskiyou female abdomen & 6 & 0.000 & 1.000 & & & & \\
\hline Siskiyou female cephalothorax & 7 & 0.110 & 0.038 & 1.000 & & & \\
\hline Siskiyou female face & 7 & 0.103 & 0.054 & 0.822 & 1.000 & & \\
\hline Siskiyou female ventrum & 7 & 0.269 & 0.012 & 0.533 & 0.442 & 1.000 & \\
\hline Siskiyou male abdomen & 2 & 0.000 & 0.001 & 0.554 & 0.812 & 0.211 & 1.000 \\
\hline Siskiyou male cephalothorax & 2 & 0.367 & 0.000 & 0.213 & 0.192 & 0.548 & 0.004 \\
\hline Siskiyou male face & 2 & 0.000 & 0.822 & 0.051 & 0.062 & 0.016 & 0.000 \\
\hline Siskiyou male ventrum & 2 & 0.020 & 0.000 & 0.530 & 0.437 & 0.868 & 0.011 \\
\hline Mt. Hood female abdomen & 13 & 0.000 & 0.144 & 0.026 & 0.030 & 0.008 & 0.000 \\
\hline Mt. Hood female cephalothorax & 9 & 0.010 & 0.005 & 0.692 & 0.917 & 0.299 & 0.852 \\
\hline Mt. Hood female face & 14 & 0.000 & 0.003 & 0.252 & 0.426 & 0.091 & 0.124 \\
\hline Mt. Hood female ventrum & 14 & 0.004 & 0.002 & 0.667 & 0.904 & 0.274 & 0.846 \\
\hline Mt. Hood male abdomen & 9 & 0.438 & 0.000 & 0.216 & 0.195 & 0.556 & 0.008 \\
\hline Mt. Hood male cephalothorax & 7 & 0.698 & 0.000 & 0.093 & 0.084 & 0.201 & 0.000 \\
\hline Mt. Hood male face & 10 & 0.000 & 0.009 & 0.173 & 0.284 & 0.063 & 0.004 \\
\hline Mt. Hood male ventrum & 10 & 0.000 & 0.179 & 0.066 & 0.108 & 0.022 & 0.000 \\
\hline Tillamook female abdomen & 9 & 0.001 & 0.518 & 0.030 & 0.039 & 0.010 & 0.032 \\
\hline Tillamook female cephalothorax & 9 & 0.003 & 0.276 & 0.076 & 0.121 & 0.027 & 0.083 \\
\hline Tillamook female face & 9 & 0.000 & 0.303 & 0.066 & 0.087 & 0.022 & 0.001 \\
\hline Tillamook female ventrum & 10 & 0.021 & 0.109 & 0.221 & 0.361 & 0.088 & 0.304 \\
\hline Tillamook male abdomen & 16 & 0.319 & 0.000 & 0.225 & 0.202 & 0.579 & 0.001 \\
\hline Tillamook male cephalothorax & 16 & 0.167 & 0.000 & 0.055 & 0.049 & 0.099 & 0.000 \\
\hline Tillamook male face & 16 & 0.000 & 0.029 & 0.128 & 0.193 & 0.046 & 0.000 \\
\hline Tillamook male ventrum & 16 & 0.000 & 0.035 & 0.103 & 0.167 & 0.036 & 0.000 \\
\hline Gorge rock & 16 & 0.001 & 0.000 & 0.668 & 0.912 & 0.269 & 0.809 \\
\hline Mt. Hood rock & 16 & 0.000 & 0.000 & 0.005 & 0.004 & 0.004 & 0.000 \\
\hline Tillamook rock & 16 & 0.081 & 0.000 & 0.325 & 0.280 & 0.797 & 0.000 \\
\hline Siskiyou rock & 11 & 0.015 & 0.000 & 0.004 & 0.004 & 0.008 & 0.000 \\
\hline Gorge dryleaf & 15 & 0.003 & 0.000 & 0.018 & 0.017 & 0.024 & 0.000 \\
\hline Siskiyou dryleaf & 4 & 0.302 & 0.030 & 0.111 & 0.096 & 0.166 & 0.088 \\
\hline
\end{tabular}


Table A - 1 (continued).

\begin{tabular}{|c|c|c|c|c|c|c|c|c|}
\hline Gorge female abdomen & 7 & & & & & & & \\
\hline Gorge female cephalothorax & 7 & & & & & & & \\
\hline Gorge female face & 7 & & & & & & & \\
\hline Gorge female ventrum & 7 & & & & & & & \\
\hline Gorge male abdomen & 4 & & & & & & & \\
\hline Gorge male cephalothorax & 3 & & & & & & & \\
\hline Gorge male face & 4 & & & & & & & \\
\hline Gorge male ventrum & 4 & & & & & & & \\
\hline Siskiyou female abdomen & 6 & & & & & & & \\
\hline Siskiyou female cephalothorax & 7 & & & & & & & \\
\hline Siskiyou female face & 7 & & & & & & & \\
\hline Siskiyou female ventrum & 7 & & & & & & & \\
\hline Siskiyou male abdomen & 2 & & & & & & & \\
\hline Siskiyou male cephalothorax & 2 & 1.000 & & & & & & \\
\hline Siskiyou male face & 2 & 0.000 & 1.000 & & & & & \\
\hline Siskiyou male ventrum & 2 & 0.191 & 0.000 & 1.000 & & & & \\
\hline Mt. Hood female abdomen & 13 & 0.000 & 0.034 & 0.000 & 1.000 & & & \\
\hline Mt. Hood female cephalothorax & 9 & 0.043 & 0.005 & 0.189 & 0.001 & 1.000 & & \\
\hline Mt. Hood female face & 14 & 0.001 & 0.001 & 0.002 & 0.000 & 0.299 & 1.000 & \\
\hline Mt. Hood female ventrum & 14 & 0.024 & 0.002 & 0.130 & 0.000 & 0.986 & 0.245 & \\
\hline Mt. Hood male abdomen & 9 & 0.984 & 0.000 & 0.245 & 0.000 & 0.052 & 0.001 & \\
\hline Mt. Hood male cephalothorax & 7 & 0.194 & 0.000 & 0.005 & 0.000 & 0.006 & 0.000 & \\
\hline Mt. Hood male face & 10 & 0.000 & 0.000 & 0.000 & 0.000 & 0.130 & 0.377 & \\
\hline Mt. Hood male ventrum & 10 & 0.000 & 0.143 & 0.000 & 0.006 & 0.019 & 0.020 & \\
\hline Tillamook female abdomen & 9 & 0.001 & 0.420 & 0.006 & 0.563 & 0.006 & 0.027 & \\
\hline Tillamook female cephalothorax & 9 & 0.005 & 0.329 & 0.018 & 0.103 & 0.043 & 0.133 & \\
\hline Tillamook female face & 9 & 0.000 & 0.239 & 0.000 & 0.014 & 0.011 & 0.006 & \\
\hline Tillamook female ventrum & 10 & 0.030 & 0.158 & 0.081 & 0.074 & 0.276 & 0.755 & \\
\hline Tillamook male abdomen & 16 & 0.938 & 0.000 & 0.206 & 0.000 & 0.045 & 0.000 & \\
\hline Tillamook male cephalothorax & 16 & 0.035 & 0.000 & 0.000 & 0.000 & 0.002 & 0.000 & \\
\hline Tillamook male face & 16 & 0.000 & 0.000 & 0.000 & 0.000 & 0.057 & 0.076 & \\
\hline Tillamook male ventrum & 16 & 0.000 & 0.004 & 0.000 & 0.000 & 0.045 & 0.064 & \\
\hline Gorge rock & 16 & 0.013 & 0.000 & 0.093 & 0.000 & 0.999 & 0.189 & \\
\hline Mt. Hood rock & 16 & 0.000 & 0.000 & 0.000 & 0.000 & 0.000 & 0.000 & \\
\hline Tillamook rock & 16 & 0.509 & 0.000 & 0.384 & 0.000 & 0.076 & 0.000 & \\
\hline Siskiyou rock & 11 & 0.006 & 0.000 & 0.001 & 0.000 & 0.000 & 0.000 & \\
\hline Gorge dryleaf & 15 & 0.001 & 0.000 & 0.000 & 0.000 & 0.000 & 0.000 & \\
\hline Siskiyou dryleaf & 4 & 0.225 & 0.032 & 0.151 & 0.024 & 0.089 & 0.065 & \\
\hline
\end{tabular}


Table A - 1 (continued).

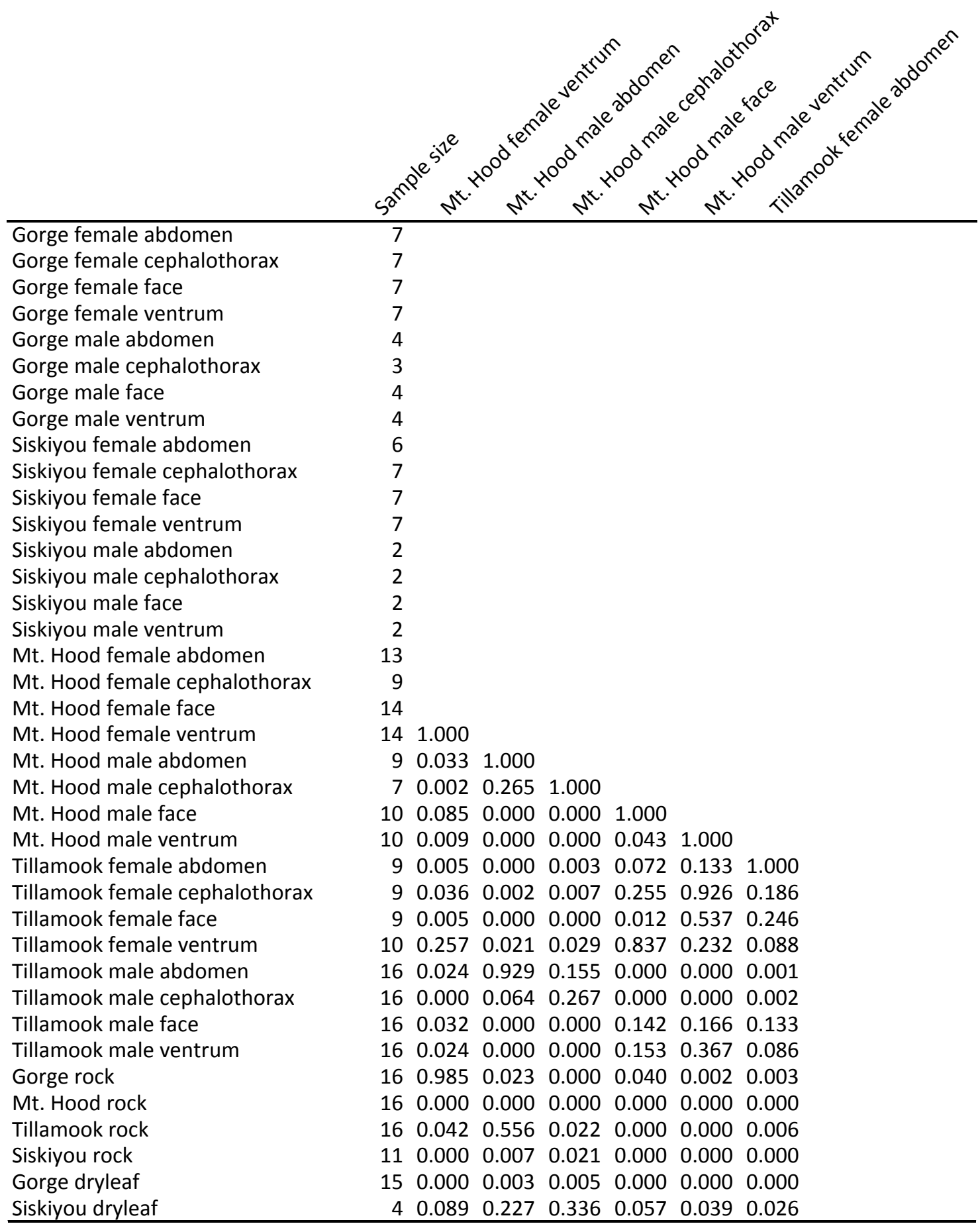


Table A - 1 (continued).

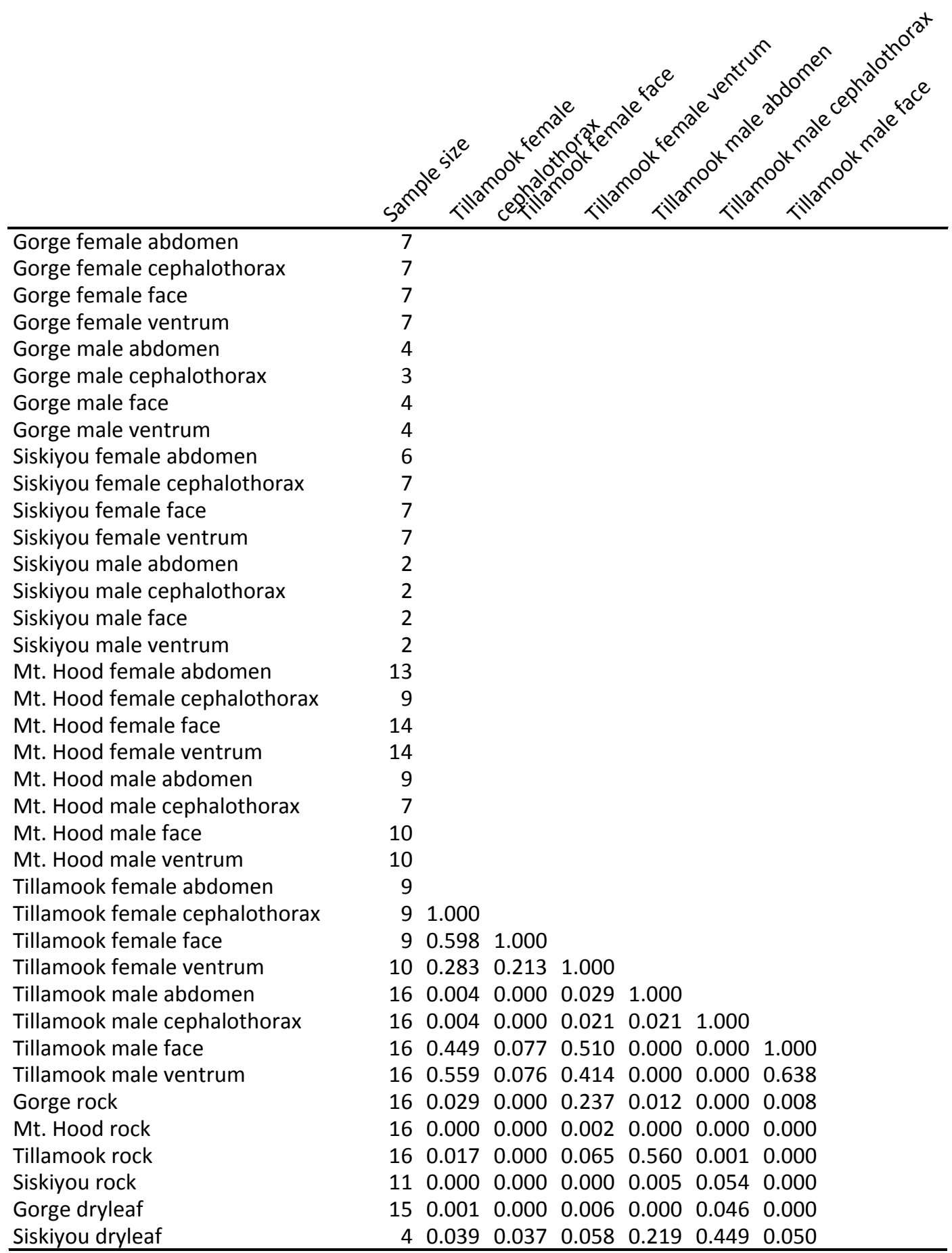


Table A - 1 (continued).

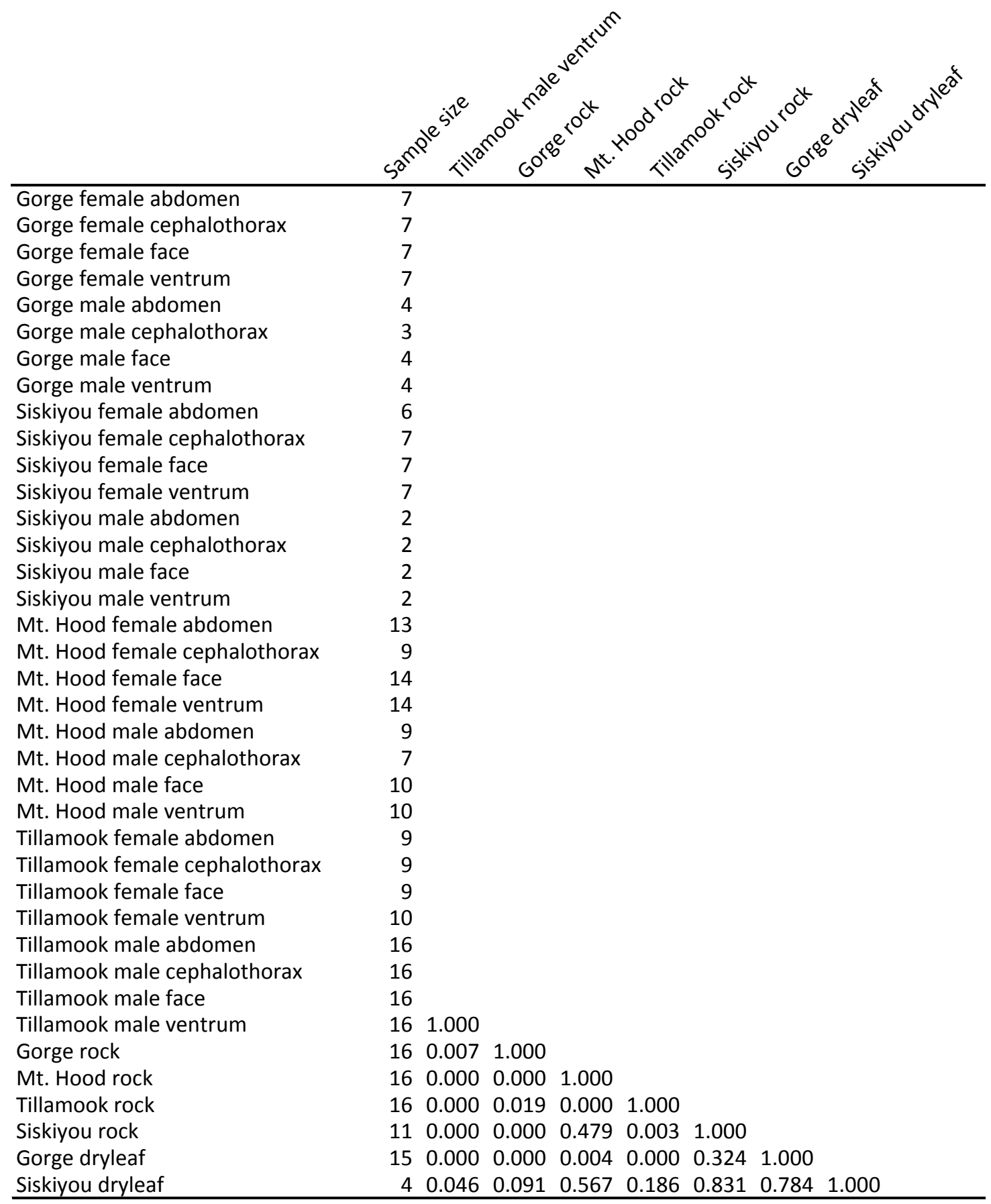


Table A - 2. Student's $t$-test comparing raw hue values of all spider reflectance spectra sorted by population, sex, and body region as well as habitat reflectance spectra grouped by population. Note that there are no Mt. Hood population leaf reflectance spectra to compare, nor are there enough Tillamook population leaf reflectance spectra to compare. Reflectance data combined with solar irradiance data. Listed below are P-values, $\alpha=0.05$. Table continued on the following pages.

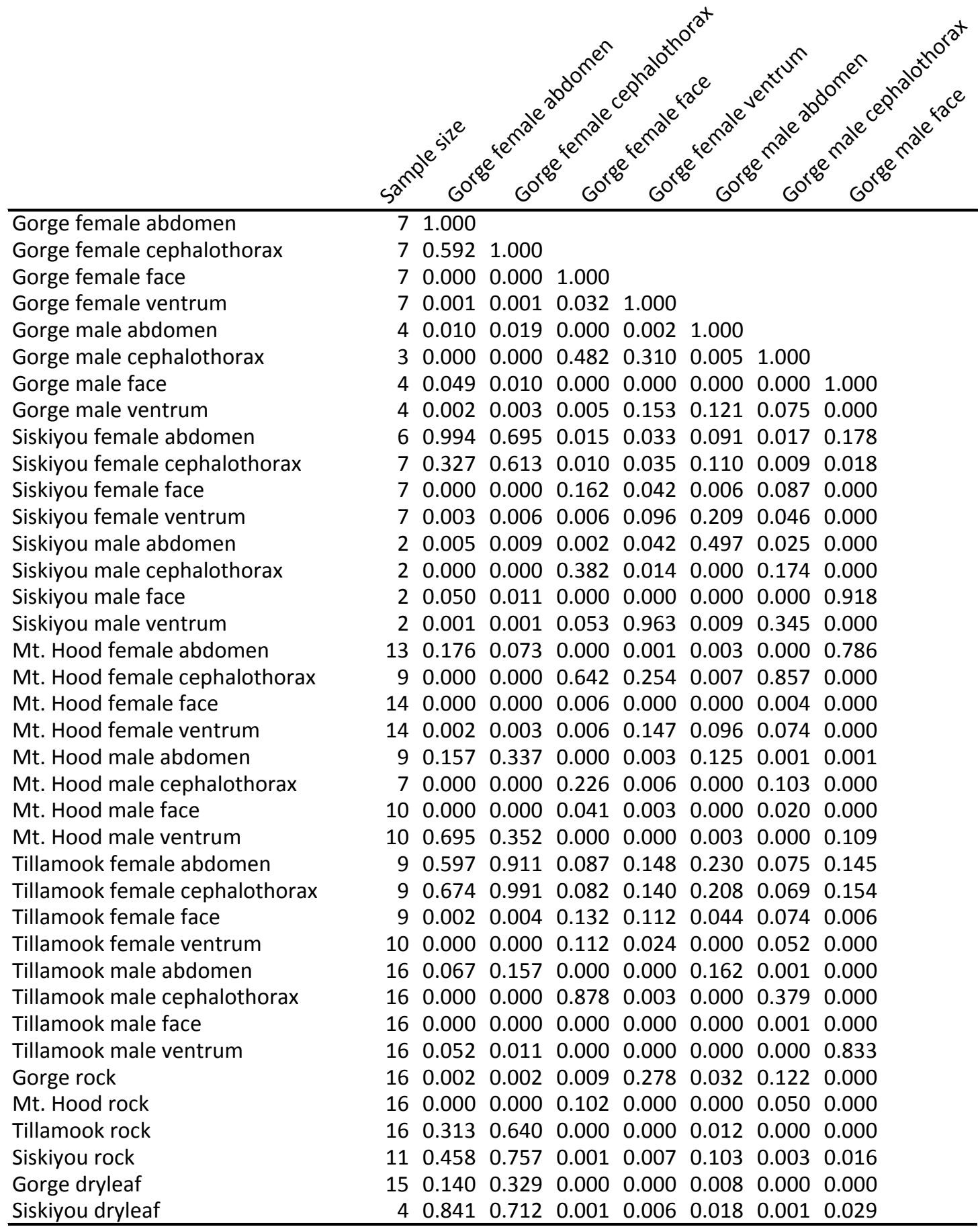


Table A - 2 (continued).

\begin{tabular}{|c|c|c|c|c|c|c|c|}
\hline Gorge female abdomen & 7 & & & & & & \\
\hline Gorge female cephalothorax & 7 & & & & & & \\
\hline Gorge female face & 7 & & & & & & \\
\hline Gorge female ventrum & 7 & & & & & & \\
\hline Gorge male abdomen & 4 & & & & & & \\
\hline Gorge male cephalothorax & 3 & & & & & & \\
\hline Gorge male face & 4 & & & & & & \\
\hline Gorge male ventrum & 4 & 1.000 & & & & & \\
\hline Siskiyou female abdomen & 6 & 0.046 & 1.000 & & & & \\
\hline Siskiyou female cephalothorax & 7 & 0.041 & 0.461 & 1.000 & & & \\
\hline Siskiyou female face & 7 & 0.016 & 0.004 & 0.003 & 1.000 & & \\
\hline Siskiyou female ventrum & 7 & 0.741 & 0.054 & 0.054 & 0.015 & 1.000 & \\
\hline Siskiyou male abdomen & 2 & 0.440 & 0.065 & 0.066 & 0.009 & 0.623 & 1.000 \\
\hline Siskiyou male cephalothorax & 2 & 0.003 & 0.009 & 0.005 & 0.382 & 0.003 & 0.001 \\
\hline Siskiyou male face & 2 & 0.000 & 0.168 & 0.015 & 0.000 & 0.000 & 0.000 \\
\hline Siskiyou male ventrum & 2 & 0.245 & 0.030 & 0.024 & 0.035 & 0.141 & 0.072 \\
\hline Mt. Hood female abdomen & 13 & 0.001 & 0.278 & 0.043 & 0.000 & 0.002 & 0.002 \\
\hline Mt. Hood female cephalothorax & 9 & 0.067 & 0.015 & 0.008 & 0.113 & 0.044 & 0.024 \\
\hline Mt. Hood female face & 14 & 0.000 & 0.006 & 0.004 & 0.707 & 0.000 & 0.000 \\
\hline Mt. Hood female ventrum & 14 & 0.959 & 0.046 & 0.044 & 0.018 & 0.688 & 0.394 \\
\hline Mt. Hood male abdomen & 9 & 0.018 & 0.305 & 0.659 & 0.001 & 0.028 & 0.059 \\
\hline Mt. Hood male cephalothorax & 7 & 0.001 & 0.008 & 0.003 & 0.534 & 0.001 & 0.000 \\
\hline Mt. Hood male face & 10 & 0.001 & 0.003 & 0.001 & 0.675 & 0.001 & 0.000 \\
\hline Mt. Hood male ventrum & 10 & 0.000 & 0.758 & 0.182 & 0.000 & 0.001 & 0.001 \\
\hline Tillamook female abdomen & 9 & 0.152 & 0.661 & 0.771 & 0.025 & 0.171 & 0.178 \\
\hline Tillamook female cephalothorax & 9 & 0.139 & 0.730 & 0.682 & 0.023 & 0.157 & 0.161 \\
\hline Tillamook female face & 9 & 0.055 & 0.005 & 0.011 & 0.436 & 0.060 & 0.039 \\
\hline Tillamook female ventrum & 10 & 0.001 & 0.011 & 0.010 & 0.564 & 0.005 & 0.001 \\
\hline Tillamook male abdomen & 16 & 0.011 & 0.211 & 0.405 & 0.001 & 0.022 & 0.066 \\
\hline Tillamook male cephalothorax & 16 & 0.001 & 0.016 & 0.014 & 0.174 & 0.003 & 0.001 \\
\hline Tillamook male face & 16 & 0.000 & 0.004 & 0.002 & 0.312 & 0.000 & 0.000 \\
\hline Tillamook male ventrum & 16 & 0.000 & 0.197 & 0.030 & 0.000 & 0.000 & 0.000 \\
\hline Gorge rock & 16 & 0.634 & 0.040 & 0.038 & 0.023 & 0.398 & 0.211 \\
\hline Mt. Hood rock & 16 & 0.000 & 0.011 & 0.008 & 0.527 & 0.000 & 0.000 \\
\hline Tillamook rock & 16 & 0.001 & 0.481 & 0.918 & 0.000 & 0.002 & 0.005 \\
\hline Siskiyou rock & 11 & 0.025 & 0.554 & 0.909 & 0.001 & 0.033 & 0.057 \\
\hline Gorge dryleaf & 15 & 0.000 & 0.330 & 0.738 & 0.002 & 0.002 & 0.004 \\
\hline Siskiyou dryleaf & 4 & 0.005 & 0.888 & 0.395 & 0.001 & 0.009 & 0.009 \\
\hline
\end{tabular}


Table A - 2 (continued).

\begin{tabular}{|c|c|c|c|c|c|c|c|c|}
\hline Gorge female abdomen & 7 & & & & & & & \\
\hline Gorge female cephalothorax & 7 & & & & & & & \\
\hline Gorge female face & 7 & & & & & & & \\
\hline Gorge female ventrum & 7 & & & & & & & \\
\hline Gorge male abdomen & 4 & & & & & & & \\
\hline Gorge male cephalothorax & 3 & & & & & & & \\
\hline Gorge male face & 4 & & & & & & & \\
\hline Gorge male ventrum & 4 & & & & & & & \\
\hline Siskiyou female abdomen & 6 & & & & & & & \\
\hline Siskiyou female cephalothorax & 7 & & & & & & & \\
\hline Siskiyou female face & 7 & & & & & & & \\
\hline Siskiyou female ventrum & 7 & & & & & & & \\
\hline Siskiyou male abdomen & 2 & & & & & & & \\
\hline Siskiyou male cephalothorax & 2 & 1.000 & & & & & & \\
\hline Siskiyou male face & 2 & 0.000 & 1.000 & & & & & \\
\hline Siskiyou male ventrum & 2 & 0.018 & 0.000 & 1.000 & & & & \\
\hline Mt. Hood female abdomen & 13 & 0.000 & 0.741 & 0.001 & 1.000 & & & \\
\hline Mt. Hood female cephalothorax & 9 & 0.261 & 0.000 & 0.281 & 0.000 & 1.000 & & \\
\hline Mt. Hood female face & 14 & 0.074 & 0.000 & 0.000 & 0.000 & 0.012 & 1.000 & \\
\hline Mt. Hood female ventrum & 14 & 0.003 & 0.000 & 0.241 & 0.001 & 0.067 & 0.000 & \\
\hline Mt. Hood male abdomen & 9 & 0.000 & 0.001 & 0.004 & 0.016 & 0.001 & 0.000 & \\
\hline Mt. Hood male cephalothorax & 7 & 0.729 & 0.000 & 0.008 & 0.000 & 0.163 & 0.157 & \\
\hline Mt. Hood male face & 10 & 0.156 & 0.000 & 0.003 & 0.000 & 0.032 & 0.886 & \\
\hline Mt. Hood male ventrum & 10 & 0.000 & 0.110 & 0.000 & 0.306 & 0.000 & 0.000 & \\
\hline Tillamook female abdomen & 9 & 0.060 & 0.126 & 0.125 & 0.146 & 0.066 & 0.061 & \\
\hline Tillamook female cephalothorax & 9 & 0.055 & 0.134 & 0.116 & 0.162 & 0.060 & 0.057 & \\
\hline Tillamook female face & 9 & 0.175 & 0.003 & 0.079 & 0.001 & 0.078 & 0.556 & \\
\hline Tillamook female ventrum & 10 & 0.562 & 0.000 & 0.006 & 0.000 & 0.096 & 0.124 & \\
\hline Tillamook male abdomen & 16 & 0.000 & 0.000 & 0.001 & 0.008 & 0.001 & 0.000 & \\
\hline Tillamook male cephalothorax & 16 & 0.402 & 0.000 & 0.017 & 0.000 & 0.543 & 0.002 & \\
\hline Tillamook male face & 16 & 0.012 & 0.000 & 0.000 & 0.000 & 0.002 & 0.260 & \\
\hline Tillamook male ventrum & 16 & 0.000 & 0.764 & 0.000 & 0.869 & 0.000 & 0.000 & \\
\hline Gorge rock & 16 & 0.004 & 0.000 & 0.418 & 0.001 & 0.107 & 0.000 & \\
\hline Mt. Hood rock & 16 & 0.624 & 0.000 & 0.000 & 0.000 & 0.102 & 0.075 & \\
\hline Tillamook rock & 16 & 0.000 & 0.001 & 0.000 & 0.031 & 0.000 & 0.000 & \\
\hline Siskiyou rock & 11 & 0.001 & 0.017 & 0.008 & 0.065 & 0.003 & 0.000 & \\
\hline Gorge dryleaf & 15 & 0.000 & 0.000 & 0.000 & 0.017 & 0.000 & 0.000 & \\
\hline Siskiyou dryleaf & 4 & 0.000 & 0.029 & 0.003 & 0.121 & 0.001 & 0.000 & \\
\hline
\end{tabular}


Table A - 2 (continued).

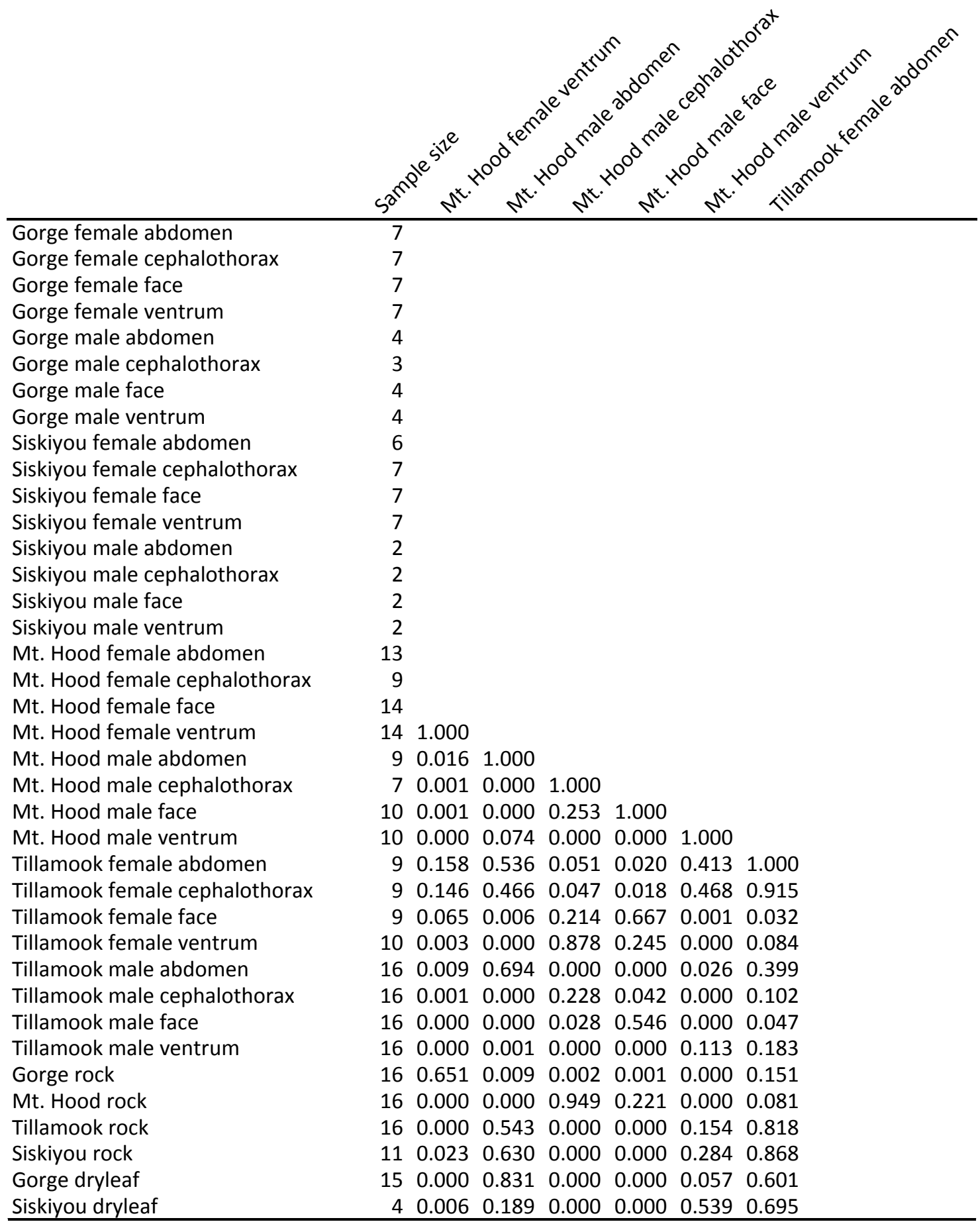


Table A - 2 (continued).

\begin{tabular}{|c|c|c|c|c|c|c|c|}
\hline Gorge female abdomen & 7 & & & & & & \\
\hline Gorge female cephalothorax & 7 & & & & & & \\
\hline Gorge female face & 7 & & & & & & \\
\hline Gorge female ventrum & 7 & & & & & & \\
\hline Gorge male abdomen & 4 & & & & & & \\
\hline Gorge male cephalothorax & 3 & & & & & & \\
\hline Gorge male face & 4 & & & & & & \\
\hline Gorge male ventrum & 4 & & & & & & \\
\hline Siskiyou female abdomen & 6 & & & & & & \\
\hline Siskiyou female cephalothorax & 7 & & & & & & \\
\hline Siskiyou female face & 7 & & & & & & \\
\hline Siskiyou female ventrum & 7 & & & & & & \\
\hline Siskiyou male abdomen & 2 & & & & & & \\
\hline Siskiyou male cephalothorax & 2 & & & & & & \\
\hline Siskiyou male face & 2 & & & & & & \\
\hline Siskiyou male ventrum & 2 & & & & & & \\
\hline Mt. Hood female abdomen & 13 & & & & & & \\
\hline Mt. Hood female cephalothorax & 9 & & & & & & \\
\hline Mt. Hood female face & 14 & & & & & & \\
\hline Mt. Hood female ventrum & 14 & & & & & & \\
\hline Mt. Hood male abdomen & 9 & & & & & & \\
\hline Mt. Hood male cephalothorax & 7 & & & & & & \\
\hline Mt. Hood male face & 10 & & & & & & \\
\hline Mt. Hood male ventrum & 10 & & & & & & \\
\hline Tillamook female abdomen & 9 & & & & & & \\
\hline Tillamook female cephalothorax & 9 & 1.000 & & & & & \\
\hline Tillamook female face & 9 & 0.029 & 1.000 & & & & \\
\hline Tillamook female ventrum & 10 & 0.080 & 0.268 & 1.000 & & & \\
\hline Tillamook male abdomen & 16 & 0.348 & 0.015 & 0.000 & 1.000 & & \\
\hline Tillamook male cephalothorax & 16 & 0.096 & 0.160 & 0.110 & 0.000 & 1.000 & \\
\hline Tillamook male face & 16 & 0.044 & 0.970 & 0.019 & 0.000 & 0.000 & 1.000 \\
\hline Tillamook male ventrum & 16 & 0.193 & 0.019 & 0.000 & 0.000 & 0.000 & 0.000 \\
\hline Gorge rock & 16 & 0.140 & 0.076 & 0.003 & 0.003 & 0.001 & 0.000 \\
\hline Mt. Hood rock & 16 & 0.077 & 0.250 & 0.903 & 0.000 & 0.066 & 0.006 \\
\hline Tillamook rock & 16 & 0.722 & 0.009 & 0.000 & 0.255 & 0.000 & 0.000 \\
\hline Siskiyou rock & 11 & 0.783 & 0.001 & 0.000 & 0.418 & 0.001 & 0.000 \\
\hline Gorge dryleaf & 15 & 0.526 & 0.032 & 0.000 & 0.425 & 0.000 & 0.000 \\
\hline Siskiyou dryleaf & 4 & 0.782 & 0.006 & 0.001 & 0.082 & 0.002 & 0.000 \\
\hline
\end{tabular}


Table A - 2 (continued).

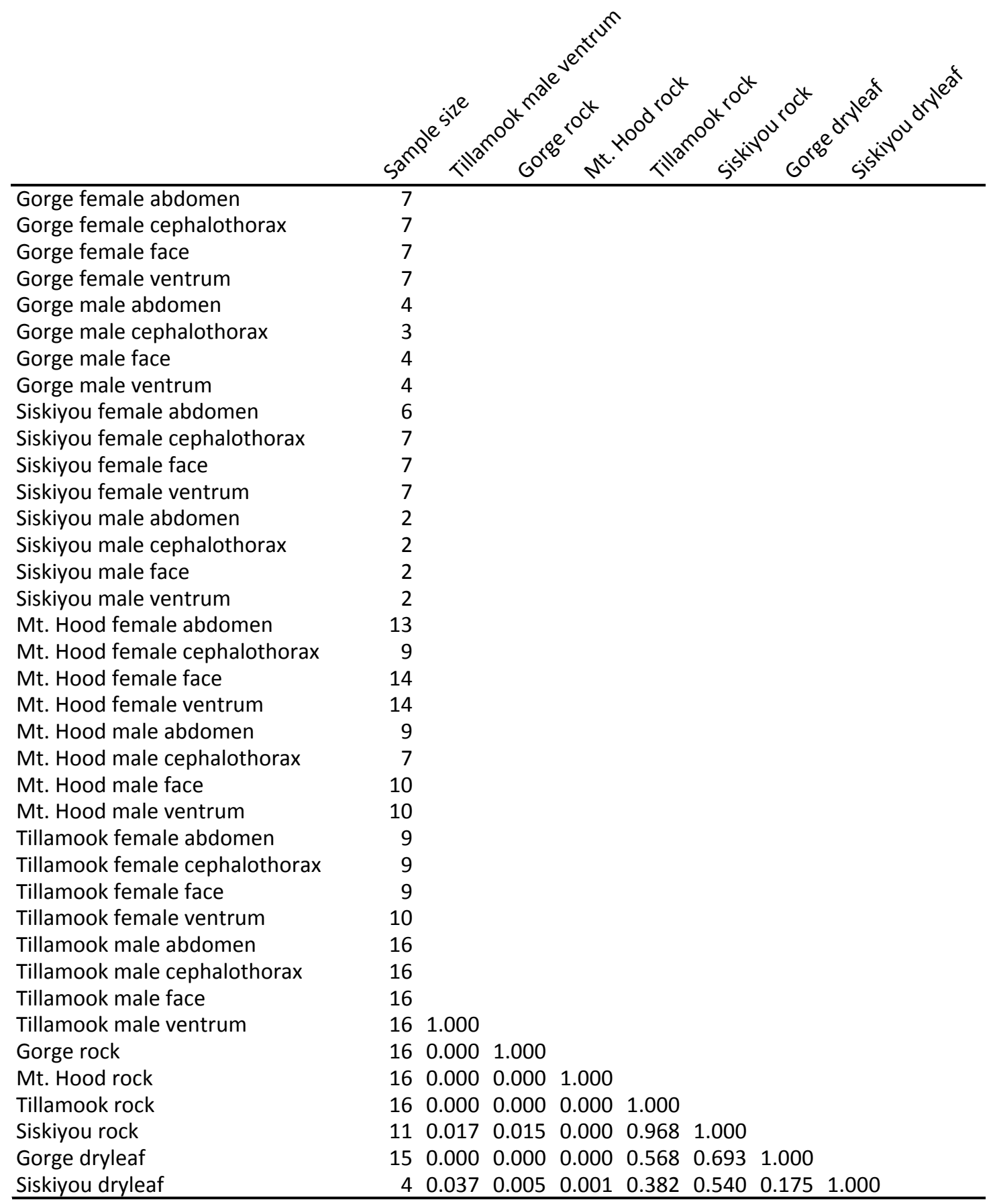


Table A - 3. Student's $t$-test comparing raw chroma values of all spider reflectance spectra sorted by population, sex, and body region as well as habitat reflectance spectra grouped by population. Note that there are no $\mathrm{Mt}$. Hood population leaf reflectance spectra to compare, nor are there enough Tillamook population leaf reflectance spectra to compare. Reflectance data combined with solar irradiance data. Listed below are P-values, $\alpha=0.05$. Table continued on the following pages.

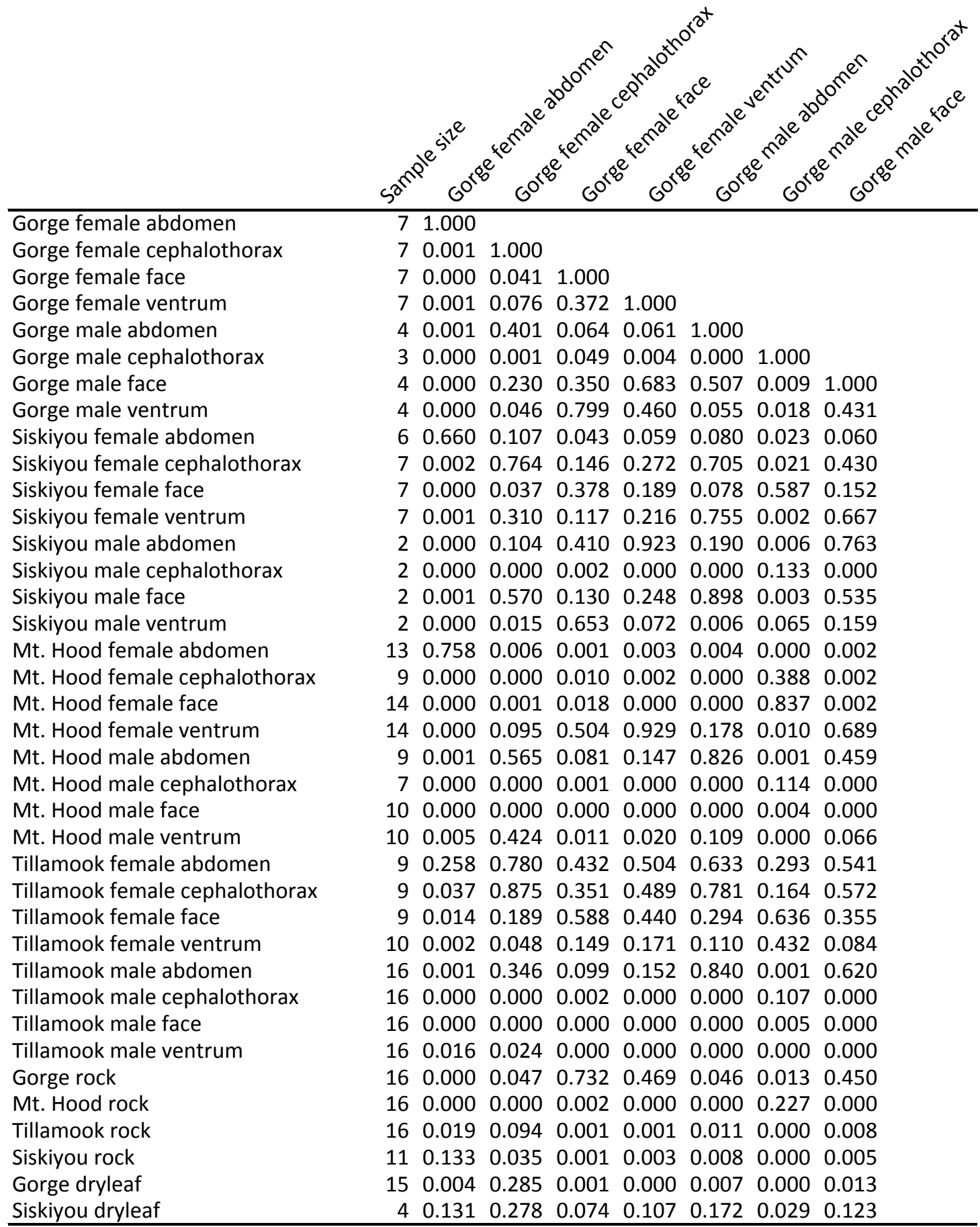


Table A - 3 (continued).

\begin{tabular}{|c|c|c|c|c|c|c|c|}
\hline Gorge female abdomen & 7 & & & & & & \\
\hline Gorge female cephalothorax & 7 & & & & & & \\
\hline Gorge female face & 7 & & & & & & \\
\hline Gorge female ventrum & 7 & & & & & & \\
\hline Gorge male abdomen & 4 & & & & & & \\
\hline Gorge male cephalothorax & 3 & & & & & & \\
\hline Gorge male face & 4 & & & & & & \\
\hline Gorge male ventrum & 4 & 1.000 & & & & & \\
\hline Siskiyou female abdomen & 6 & 0.048 & 1.000 & & & & \\
\hline Siskiyou female cephalothorax & 7 & 0.176 & 0.092 & 1.000 & & & \\
\hline Siskiyou female face & 7 & 0.296 & 0.025 & 0.074 & 1.000 & & \\
\hline Siskiyou female ventrum & 7 & 0.126 & 0.074 & 0.585 & 0.095 & 1.000 & \\
\hline Siskiyou male abdomen & 2 & 0.517 & 0.057 & 0.289 & 0.184 & 0.338 & 1.000 \\
\hline Siskiyou male cephalothorax & 2 & 0.000 & 0.016 & 0.009 & 0.149 & 0.000 & 0.000 \\
\hline Siskiyou male face & 2 & 0.155 & 0.080 & 0.820 & 0.074 & 0.741 & 0.318 \\
\hline Siskiyou male ventrum & 2 & 0.418 & 0.040 & 0.101 & 0.509 & 0.027 & 0.140 \\
\hline Mt. Hood female abdomen & 13 & 0.002 & 0.532 & 0.005 & 0.001 & 0.004 & 0.002 \\
\hline Mt. Hood female cephalothorax & 9 & 0.003 & 0.018 & 0.011 & 0.274 & 0.001 & 0.001 \\
\hline Mt. Hood female face & 14 & 0.002 & 0.024 & 0.029 & 0.487 & 0.001 & 0.001 \\
\hline Mt. Hood female ventrum & 14 & 0.635 & 0.054 & 0.263 & 0.208 & 0.302 & 0.888 \\
\hline Mt. Hood male abdomen & 9 & 0.091 & 0.083 & 0.842 & 0.065 & 0.652 & 0.224 \\
\hline Mt. Hood male cephalothorax & 7 & 0.000 & 0.016 & 0.011 & 0.148 & 0.000 & 0.000 \\
\hline Mt. Hood male face & 10 & 0.000 & 0.011 & 0.005 & 0.038 & 0.000 & 0.000 \\
\hline Mt. Hood male ventrum & 10 & 0.012 & 0.171 & 0.320 & 0.014 & 0.084 & 0.027 \\
\hline Tillamook female abdomen & 9 & 0.455 & 0.340 & 0.706 & 0.321 & 0.601 & 0.508 \\
\hline Tillamook female cephalothorax & 9 & 0.393 & 0.112 & 0.963 & 0.188 & 0.703 & 0.494 \\
\hline Tillamook female face & 9 & 0.526 & 0.034 & 0.222 & 0.933 & 0.315 & 0.419 \\
\hline Tillamook female ventrum & 10 & 0.150 & 0.015 & 0.058 & 0.286 & 0.112 & 0.132 \\
\hline Tillamook male abdomen & 16 & 0.103 & 0.075 & 0.628 & 0.087 & 0.923 & 0.298 \\
\hline Tillamook male cephalothorax & 16 & 0.000 & 0.019 & 0.021 & 0.168 & 0.000 & 0.000 \\
\hline Tillamook male face & 16 & 0.000 & 0.013 & 0.009 & 0.051 & 0.000 & 0.000 \\
\hline Tillamook male ventrum & 16 & 0.000 & 0.317 & 0.068 & 0.007 & 0.000 & 0.000 \\
\hline Gorge rock & 16 & 0.932 & 0.050 & 0.187 & 0.275 & 0.122 & 0.543 \\
\hline Mt. Hood rock & 16 & 0.000 & 0.019 & 0.016 & 0.217 & 0.000 & 0.000 \\
\hline Tillamook rock & 16 & 0.001 & 0.301 & 0.085 & 0.004 & 0.009 & 0.002 \\
\hline Siskiyou rock & 11 & 0.001 & 0.568 & 0.032 & 0.002 & 0.007 & 0.003 \\
\hline Gorge dryleaf & 15 & 0.000 & 0.170 & 0.250 & 0.016 & 0.011 & 0.001 \\
\hline Siskiyou dryleaf & 4 & 0.083 & 0.402 & 0.229 & 0.039 & 0.154 & 0.107 \\
\hline
\end{tabular}


Table A - 3 (continued).

\begin{tabular}{|c|c|c|c|c|c|c|c|c|}
\hline Gorge female abdomen & 7 & & & & & & & \\
\hline Gorge female cephalothorax & 7 & & & & & & & \\
\hline Gorge female face & 7 & & & & & & & \\
\hline Gorge female ventrum & 7 & & & & & & & \\
\hline Gorge male abdomen & 4 & & & & & & & \\
\hline Gorge male cephalothorax & 3 & & & & & & & \\
\hline Gorge male face & 4 & & & & & & & \\
\hline Gorge male ventrum & 4 & & & & & & & \\
\hline Siskiyou female abdomen & 6 & & & & & & & \\
\hline Siskiyou female cephalothorax & 7 & & & & & & & \\
\hline Siskiyou female face & 7 & & & & & & & \\
\hline Siskiyou female ventrum & 7 & & & & & & & \\
\hline Siskiyou male abdomen & 2 & & & & & & & \\
\hline Siskiyou male cephalothorax & 2 & 1.000 & & & & & & \\
\hline Siskiyou male face & 2 & 0.000 & 1.000 & & & & & \\
\hline Siskiyou male ventrum & 2 & 0.002 & 0.051 & 1.000 & & & & \\
\hline Mt. Hood female abdomen & 13 & 0.000 & 0.003 & 0.001 & 1.000 & & & \\
\hline Mt. Hood female cephalothorax & 9 & 0.530 & 0.001 & 0.012 & 0.000 & 1.000 & & \\
\hline Mt. Hood female face & 14 & 0.110 & 0.001 & 0.012 & 0.001 & 0.408 & 1.000 & \\
\hline Mt. Hood female ventrum & 14 & 0.000 & 0.286 & 0.213 & 0.002 & 0.002 & 0.002 & \\
\hline Mt. Hood male abdomen & 9 & 0.000 & 0.960 & 0.022 & 0.004 & 0.000 & 0.000 & \\
\hline Mt. Hood male cephalothorax & 7 & 0.981 & 0.000 & 0.000 & 0.000 & 0.518 & 0.079 & \\
\hline Mt. Hood male face & 10 & 0.093 & 0.000 & 0.000 & 0.000 & 0.034 & 0.001 & \\
\hline Mt. Hood male ventrum & 10 & 0.000 & 0.200 & 0.004 & 0.012 & 0.000 & 0.000 & \\
\hline Tillamook female abdomen & 9 & 0.237 & 0.653 & 0.403 & 0.210 & 0.256 & 0.293 & \\
\hline Tillamook female cephalothorax & 9 & 0.117 & 0.839 & 0.311 & 0.025 & 0.127 & 0.183 & \\
\hline Tillamook female face & 9 & 0.320 & 0.248 & 0.710 & 0.008 & 0.414 & 0.585 & \\
\hline Tillamook female ventrum & 10 & 0.954 & 0.057 & 0.196 & 0.001 & 0.749 & 0.484 & \\
\hline Tillamook male abdomen & 16 & 0.000 & 0.796 & 0.015 & 0.004 & 0.000 & 0.000 & \\
\hline Tillamook male cephalothorax & 16 & 0.810 & 0.000 & 0.000 & 0.001 & 0.580 & 0.045 & \\
\hline Tillamook male face & 16 & 0.130 & 0.000 & 0.000 & 0.000 & 0.047 & 0.000 & \\
\hline Tillamook male ventrum & 16 & 0.000 & 0.005 & 0.000 & 0.035 & 0.000 & 0.000 & \\
\hline Gorge rock & 16 & 0.000 & 0.159 & 0.336 & 0.002 & 0.002 & 0.001 & \\
\hline Mt. Hood rock & 16 & 0.591 & 0.000 & 0.001 & 0.000 & 0.839 & 0.180 & \\
\hline Tillamook rock & 16 & 0.000 & 0.034 & 0.000 & 0.032 & 0.000 & 0.000 & \\
\hline Siskiyou rock & 11 & 0.000 & 0.015 & 0.001 & 0.122 & 0.000 & 0.000 & \\
\hline Gorge dryleaf & 15 & 0.000 & 0.093 & 0.000 & 0.013 & 0.000 & 0.000 & \\
\hline Siskiyou dryleaf & 4 & 0.017 & 0.188 & 0.064 & 0.107 & 0.020 & 0.031 & \\
\hline
\end{tabular}


Table A - 3 (continued).

\begin{tabular}{|c|c|c|c|c|c|c|c|c|}
\hline Gorge female abdomen & 7 & & & & & & & \\
\hline Gorge female cephalothorax & 7 & & & & & & & \\
\hline Gorge female face & 7 & & & & & & & \\
\hline Gorge female ventrum & 7 & & & & & & & \\
\hline Gorge male abdomen & 4 & & & & & & & \\
\hline Gorge male cephalothorax & 3 & & & & & & & \\
\hline Gorge male face & 4 & & & & & & & \\
\hline Gorge male ventrum & 4 & & & & & & & \\
\hline Siskiyou female abdomen & 6 & & & & & & & \\
\hline Siskiyou female cephalothorax & 7 & & & & & & & \\
\hline Siskiyou female face & 7 & & & & & & & \\
\hline Siskiyou female ventrum & 7 & & & & & & & \\
\hline Siskiyou male abdomen & 2 & & & & & & & \\
\hline Siskiyou male cephalothorax & 2 & & & & & & & \\
\hline Siskiyou male face & 2 & & & & & & & \\
\hline Siskiyou male ventrum & 2 & & & & & & & \\
\hline Mt. Hood female abdomen & 13 & & & & & & & \\
\hline Mt. Hood female cephalothorax & 9 & & & & & & & \\
\hline Mt. Hood female face & 14 & & & & & & & \\
\hline Mt. Hood female ventrum & 14 & 1.000 & & & & & & \\
\hline Mt. Hood male abdomen & 9 & 0.202 & 1.000 & & & & & \\
\hline Mt. Hood male cephalothorax & 7 & 0.000 & 0.000 & 1.000 & & & & \\
\hline Mt. Hood male face & 10 & 0.000 & 0.000 & 0.062 & 1.000 & & & \\
\hline Mt. Hood male ventrum & 10 & 0.025 & 0.182 & 0.000 & 0.000 & 1.000 & & \\
\hline Tillamook female abdomen & 9 & 0.495 & 0.662 & 0.241 & 0.200 & 0.992 & 1.000 & \\
\hline Tillamook female cephalothorax & 9 & 0.468 & 0.858 & 0.127 & 0.096 & 0.511 & 0.729 & \\
\hline Tillamook female face & 9 & 0.438 & 0.254 & 0.328 & 0.198 & 0.113 & 0.330 & \\
\hline Tillamook female ventrum & 10 & 0.129 & 0.073 & 0.964 & 0.389 & 0.023 & 0.219 & \\
\hline Tillamook male abdomen & 16 & 0.268 & 0.715 & 0.000 & 0.000 & 0.095 & 0.611 & \\
\hline Tillamook male cephalothorax & 16 & 0.000 & 0.000 & 0.808 & 0.017 & 0.000 & 0.252 & \\
\hline Tillamook male face & 16 & 0.000 & 0.000 & 0.083 & 0.684 & 0.000 & 0.210 & \\
\hline Tillamook male ventrum & 16 & 0.000 & 0.002 & 0.000 & 0.000 & 0.211 & 0.696 & \\
\hline Gorge rock & 16 & 0.672 & 0.090 & 0.000 & 0.000 & 0.012 & 0.462 & \\
\hline Mt. Hood rock & 16 & 0.000 & 0.000 & 0.572 & 0.014 & 0.000 & 0.257 & \\
\hline Tillamook rock & 16 & 0.002 & 0.026 & 0.000 & 0.000 & 0.394 & 0.732 & \\
\hline Siskiyou rock & 11 & 0.002 & 0.013 & 0.000 & 0.000 & 0.137 & 0.509 & \\
\hline Gorge dryleaf & 15 & 0.001 & 0.059 & 0.000 & 0.000 & 0.994 & 0.990 & \\
\hline Siskiyou dryleaf & 4 & 0.100 & 0.191 & 0.018 & 0.011 & 0.510 & 0.691 & \\
\hline
\end{tabular}


Table A - 3 (continued).

\begin{tabular}{|c|c|c|c|c|c|c|c|}
\hline Gorge female abdomen & 7 & & & & & & \\
\hline Gorge female cephalothorax & 7 & & & & & & \\
\hline Gorge female face & 7 & & & & & & \\
\hline Gorge female ventrum & 7 & & & & & & \\
\hline Gorge male abdomen & 4 & & & & & & \\
\hline Gorge male cephalothorax & 3 & & & & & & \\
\hline Gorge male face & 4 & & & & & & \\
\hline Gorge male ventrum & 4 & & & & & & \\
\hline Siskiyou female abdomen & 6 & & & & & & \\
\hline Siskiyou female cephalothorax & 7 & & & & & & \\
\hline Siskiyou female face & 7 & & & & & & \\
\hline Siskiyou female ventrum & 7 & & & & & & \\
\hline Siskiyou male abdomen & 2 & & & & & & \\
\hline Siskiyou male cephalothorax & 2 & & & & & & \\
\hline Siskiyou male face & 2 & & & & & & \\
\hline Siskiyou male ventrum & 2 & & & & & & \\
\hline Mt. Hood female abdomen & 13 & & & & & & \\
\hline Mt. Hood female cephalothorax & 9 & & & & & & \\
\hline Mt. Hood female face & 14 & & & & & & \\
\hline Mt. Hood female ventrum & 14 & & & & & & \\
\hline Mt. Hood male abdomen & 9 & & & & & & \\
\hline Mt. Hood male cephalothorax & 7 & & & & & & \\
\hline Mt. Hood male face & 10 & & & & & & \\
\hline Mt. Hood male ventrum & 10 & & & & & & \\
\hline Tillamook female abdomen & 9 & & & & & & \\
\hline Tillamook female cephalothorax & 9 & 1.000 & & & & & \\
\hline Tillamook female face & 9 & 0.256 & 1.000 & & & & \\
\hline Tillamook female ventrum & 10 & 0.102 & 0.348 & 1.000 & & & \\
\hline Tillamook male abdomen & 16 & 0.729 & 0.303 & 0.103 & 1.000 & & \\
\hline Tillamook male cephalothorax & 16 & 0.154 & 0.362 & 0.958 & 0.000 & 1.000 & \\
\hline Tillamook male face & 16 & 0.113 & 0.226 & 0.461 & 0.000 & 0.010 & 1.000 \\
\hline Tillamook male ventrum & 16 & 0.281 & 0.126 & 0.059 & 0.000 & 0.000 & 0.000 \\
\hline Gorge rock & 16 & 0.408 & 0.512 & 0.157 & 0.096 & 0.000 & 0.000 \\
\hline Mt. Hood rock & 16 & 0.145 & 0.391 & 0.826 & 0.000 & 0.642 & 0.013 \\
\hline Tillamook rock & 16 & 0.264 & 0.070 & 0.013 & 0.009 & 0.000 & 0.000 \\
\hline Siskiyou rock & 11 & 0.126 & 0.030 & 0.004 & 0.007 & 0.000 & 0.000 \\
\hline Gorge dryleaf & 15 & 0.506 & 0.165 & 0.069 & 0.007 & 0.000 & 0.000 \\
\hline Siskiyou dryleaf & 4 & 0.296 & 0.068 & 0.020 & 0.160 & 0.022 & 0.013 \\
\hline
\end{tabular}


Table A - 3 (continued).

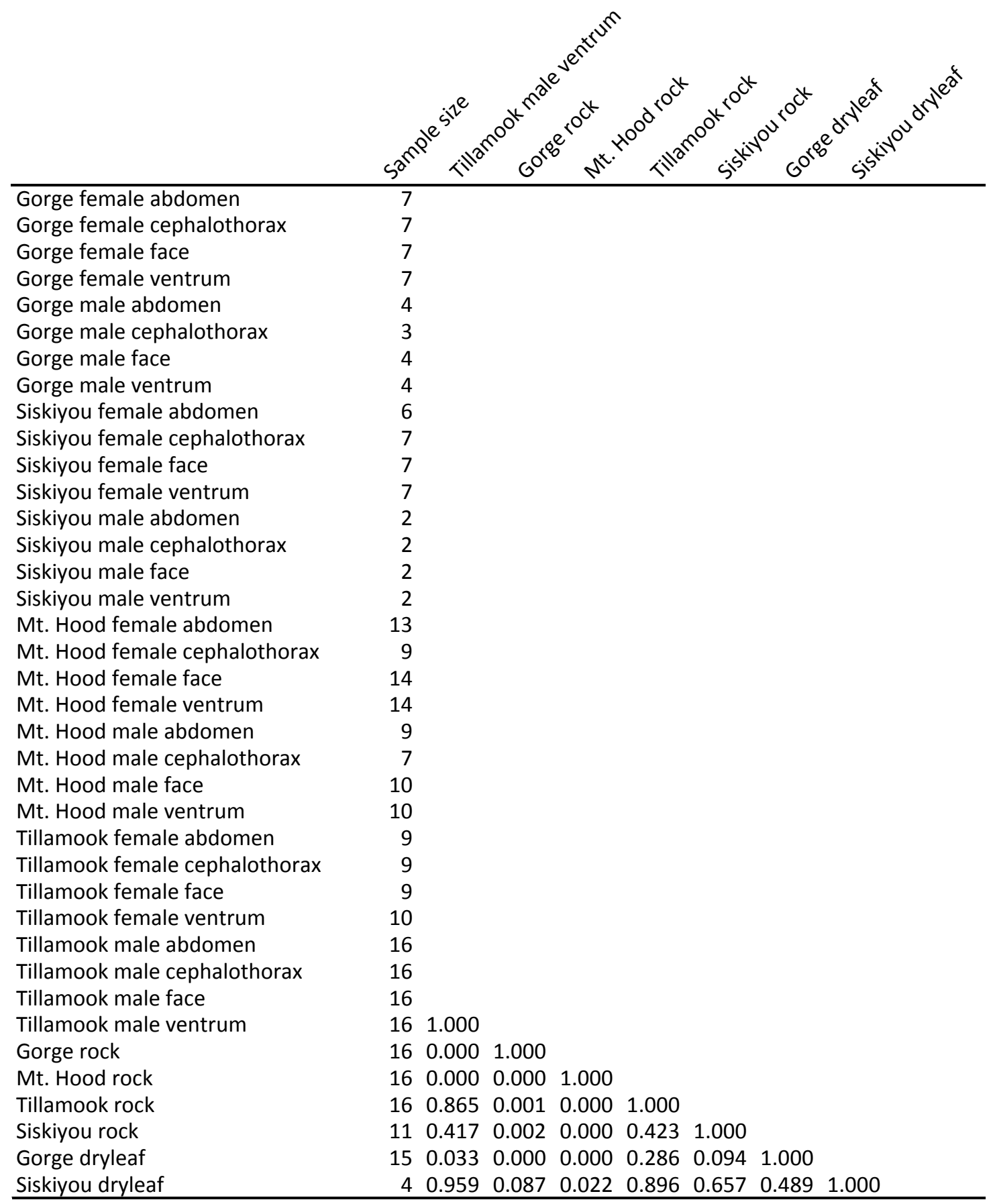

